\title{
ATLAS Run 1 searches for direct pair production of third-generation squarks at the Large Hadron Collider
}

\author{
ATLAS Collaboration ${ }^{\star}$ \\ CERN, 1211 Geneva 23, Switzerland
}

Received: 30 June 2015 / Accepted: 8 October 2015 / Published online: 29 October 2015

(C) CERN for the benefit of the ATLAS collaboration 2015. This article is published with open access at Springerlink.com

\begin{abstract}
This paper reviews and extends searches for the direct pair production of the scalar supersymmetric partners of the top and bottom quarks in proton-proton collisions collected by the ATLAS collaboration during the LHC Run 1 . Most of the analyses use $20 \mathrm{fb}^{-1}$ of collisions at a centreof-mass energy of $\sqrt{s}=8 \mathrm{TeV}$, although in some case an additional $4.7 \mathrm{fb}^{-1}$ of collision data at $\sqrt{s}=7 \mathrm{TeV}$ are used. New analyses are introduced to improve the sensitivity to specific regions of the model parameter space. Since no evidence of third-generation squarks is found, exclusion limits are derived by combining several analyses and are presented in both a simplified model framework, assuming simple decay chains, as well as within the context of more elaborate phenomenological supersymmetric models.
\end{abstract}

\section{Contents}

1 Introduction . . . . . . . . . . . . . 1

2 Third-generation squark phenomenology ... . . . 2

3 General discussion of the analysis strategy . . . . . 4

4 Interpretations in simplified models . . . . . . . . 5

4.1 Stop decays with no charginos in the decay chain 5

4.2 Stop decays with a chargino in the decay chain . 8

4.3 Limits on pair production of $\tilde{t}_{2} \ldots \ldots$

4.4 Sbottom decays . . . . . . . . . . . . . . 11

5 Interpretations in pMSSM models . . . . . . . . . 11

6 Conclusions . . . . . . . . . . . . . . 17

A The ATLAS detector and object reconstruction . . . 18

B Analyses used in the paper . . . . . . . . . . . . . 19

B.1 Review of already published signal regions . . . 19

B.2 Description of the new signal regions . . . . . 22

B.2.1 Final states with two leptons at intermediate values of $m_{\mathrm{T} 2}(\mathrm{WW}) \ldots \ldots 23$

B.2.2 Final states containing two top quarks and a Higgs boson (t2t1h) . . . . . . . . 25

^e-mail: atlas.publications@cern.ch
B.2.3 Final states containing two $b$-jets, a charged lepton, and missing transverse momentum (tb) 27

C Further details of the statistical combination of the $\mathrm{t} 0 \mathrm{~L}$ and $\mathrm{t} 1 \mathrm{~L}$ signal regions $\ldots \ldots 30$

D Signal generation details . . . . . . . . . . . . . 33

References . . . . . . . . . . . . . . . . . . . 34

\section{Introduction}

In a theory with broken supersymmetry (SUSY) [1-9], the mass scale of the supersymmetric particles is undetermined. However, for SUSY to provide a solution to the hierarchy problem [10-13] some of the new SUSY particles masses are typically required to be below about one $\mathrm{TeV}[14,15]$, hence they could be within the reach of the LHC.

The scalar partners of the right-handed and left-handed chiral components of the top-quark state $\left(\tilde{t}_{\mathrm{R}}\right.$ and $\tilde{t}_{\mathrm{L}}$ respectively) are among these particles. In many supersymmetric models, the large Yukawa coupling of the top quark to the Higgs sector makes the Higgs boson mass sensitive to the masses of the scalar top (referred to as stop in the following) states, such that, to avoid fine tuning, their masses are often required to be light. The $\tilde{t}_{\mathrm{R}}$ and $\tilde{t}_{\mathrm{L}}$ components mix to form the mass eigenstates $\tilde{t}_{1}$ and $\tilde{t}_{2}, \tilde{t}_{1}$ being defined as the lighter of the two. The scalar superpartner of the lefthanded chiral component of the bottom quark $\left(\tilde{b}_{\mathrm{L}}\right)$ belongs to the same weak isospin doublet as the $\tilde{t}_{\mathrm{L}}$, hence they usually share the same supersymmetry-breaking mass parameter: a light stop can therefore imply the existence of a light scalar bottom. The lightest sbottom mass eigenstate is referred to as $\tilde{b}_{1}$.

The ATLAS and CMS collaborations have searched for direct production of stops and sbottoms [16-35] using about $4.7 \mathrm{fb}^{-1}$ of data from the proton-proton collisions produced by the LHC at $\sqrt{s}=7 \mathrm{TeV}$ and $20 \mathrm{fb}^{-1}$ at $\sqrt{s}=8 \mathrm{TeV}$. These searches have found no evidence of third-generation squark signals, leading to exclusion limits in many SUSY models. The aim of this paper is to sum- 
marise the sensitivity of the ATLAS experiment to R-parityconserving $^{1}$ [38-42] models including the direct pair production of stops and sbottoms using the full $\sqrt{s}=8 \mathrm{TeV}$ proton-proton collision dataset collected during Run 1 of the LHC. $^{2}$ The third-generation squarks are assumed to decay to the stable lightest supersymmetric particle (LSP) directly or through one or more intermediate stages. The analyses considered are those previously published by the ATLAS collaboration on the topic, together with new ones designed to increase the sensitivity to scenarios not optimally covered so far. A wide range of SUSY scenarios are studied by combining different analyses to improve the global sensitivity.

The paper is organised as follows: Sect. 2 briefly reviews the expected phenomenology of third-generation squark production and decay; Sect. 3 reviews the general analysis approach followed by the ATLAS collaboration for SUSY searches; Sects. 4 and 5 present the exclusion limits obtained in specific models by combining the results of several analyses. Two different types of models have been considered: simplified models, where the third-generation squarks are assumed to decay into typically one or two different final states, and more complex phenomenological supersymmetric models, where the stop and sbottom have many allowed decay channels. Conclusions are drawn in Sect. 6.

For the sake of brevity, the body of the paper provides no details of the ATLAS detector and object reconstruction, of the analyses used in the limit derivation, or of how the signal Monte Carlo simulation samples were generated. However, a comprehensive set of appendices is provided to supply additional information to the interested reader. Appendix A briefly summarises the layout of the ATLAS detector and the general principles used in the reconstruction of electrons, muons, jets, jets containing $b$-hadrons ( $b$-jets), and the missing transverse momentum vector $\mathbf{p}_{\mathrm{T}}^{\text {miss }}$ (whose magnitude is referred to as $E_{\mathrm{T}}^{\text {miss }}$ ). Appendix B discusses the analyses used to derive the exclusion limits presented in Sects. 4 and 5 . The analyses that have already been published are only briefly reviewed, while those presented for the first time in this paper are discussed in detail. Appendix $\mathrm{C}$ provides further details of a combination of analyses which is performed for the first time in this paper. Finally, Appendix D provides details about the generation and simulation of the signal Monte Carlo samples used to derive the limits presented.

\footnotetext{
${ }^{1}$ It is also assumed that the decay of the third-generation squarks is prompt: long-lived and metastable stops/sbottoms are discussed elsewhere [36,37].

2 The analysis exploiting the measurement of the $t \bar{t}$ cross section discussed in this paper also uses $4.7 \mathrm{fb}^{-1}$ of proton-proton collisions at $\sqrt{s}=7 \mathrm{TeV}$.
}

\section{Third-generation squark phenomenology}

The cross section for direct stop pair production in protonproton collisions at $\sqrt{s}=8 \mathrm{TeV}$ as a function of the stop mass as calculated with PROSPINO [43,44] is shown in Fig. 1a. It is calculated to next-to-leading order accuracy in the strong coupling constant, adding the resummation of soft gluon emission at next-to-leading-logarithmic accuracy (NLO+NLL) [45-47]. In this paper, the nominal cross section and its uncertainty are taken from an envelope of cross-section predictions using different parton distribution function (PDF) sets and factorisation and renormalisation scales described in Ref. [44]. The difference in cross section between the sbottom and stop pair production is known to be small [46], hence the values of Fig. 1a are used for both.

Searches for direct production of stops and sbottoms by the ATLAS collaboration have covered several possible finalstate topologies. The experimental signatures used to identify these processes depend on the masses of the stop or sbottom, on the masses of the other supersymmetric particles they can decay into, and on other parameters of the model, such as the stop and sbottom left-right mixing and the mixing between the gaugino and higgsino states in the chargino-neutralino sector.

Assuming that the lightest supersymmetric particle is a stable neutralino $\left(\tilde{\chi}_{1}^{0}\right)$, and that no other supersymmetric particle plays a significant role in the sbottom decay, the decay chain of the sbottom is simply $\tilde{b}_{1} \rightarrow b \tilde{\chi}_{1}^{0}$ (Fig. 2a).

A significantly more complex phenomenology has to be considered for the stop, depending on its mass and on the $\tilde{\chi}_{1}^{0}$ mass. Figure $1 \mathrm{~b}$ shows the three main regions in the $m_{\tilde{t}_{1}}$ $m_{\tilde{\chi}_{1}^{0}}$ plane that are taken into account. They are identified by different values of $\Delta m\left(\tilde{t}_{1}, \tilde{\chi}_{1}^{0}\right)=m_{\tilde{t}_{1}}-m_{\tilde{\chi}_{1}^{0}}$. In the region where $\Delta m\left(\tilde{t}_{1}, \tilde{\chi}_{1}^{0}\right)>m_{t}$, the favoured decay is $\tilde{t}_{1} \rightarrow t \tilde{\chi}_{1}^{0}$ (Fig. 2b). The region where $m_{W}+m_{b}<\Delta m\left(\tilde{t}_{1}, \tilde{\chi}_{1}^{0}\right)<m_{t}$ is characterised by the three-body decay ${ }^{3}\left(\tilde{t}_{1} \rightarrow W b \tilde{\chi}_{1}^{0}\right.$ through an off-shell top quark, Fig. 2c). The region where the value of $\Delta m\left(\tilde{t}_{1}, \tilde{\chi}_{1}^{0}\right)$ drops below $m_{W}+m_{b}$, sees the four-body decay $\tilde{t}_{1} \rightarrow b f f^{\prime} \tilde{\chi}_{1}^{0}$, (where $f$ and $f^{\prime}$ indicate generic fermions coming from the decay of an off-shell $W$ boson, Fig. 2d) competing with the flavour-changing decay ${ }^{4} \tilde{t}_{1} \rightarrow c \tilde{\chi}_{1}^{0}$ of Fig. 2e; the dominant decay depends on the details of the supersymmetric model chosen [50].

If the third-generation squark decay involves more SUSY particles (other than the $\tilde{\chi}_{1}^{0}$ ), then additional dependencies on

\footnotetext{
${ }^{3}$ In scenarios that depart from the minimal flavour violation assumption, flavour-changing decays like $\tilde{t}_{1} \rightarrow c \tilde{\chi}_{1}^{0}$ or $\tilde{t}_{1} \rightarrow u \tilde{\chi}_{1}^{0}$ could have a significant branching ratio up to $\Delta m\left(\tilde{t}_{1}, \tilde{\chi}_{1}^{0}\right) \sim 100 \mathrm{GeV}$ [48].

4 The decay $\tilde{t}_{1} \rightarrow u \tilde{\chi}_{1}^{0}$, in the assumption of minimal flavour violation [49], is further suppressed with respect to $\tilde{t}_{1} \rightarrow c \tilde{\chi}_{1}^{0}$ by corresponding factors of the CKM matrix.
} 


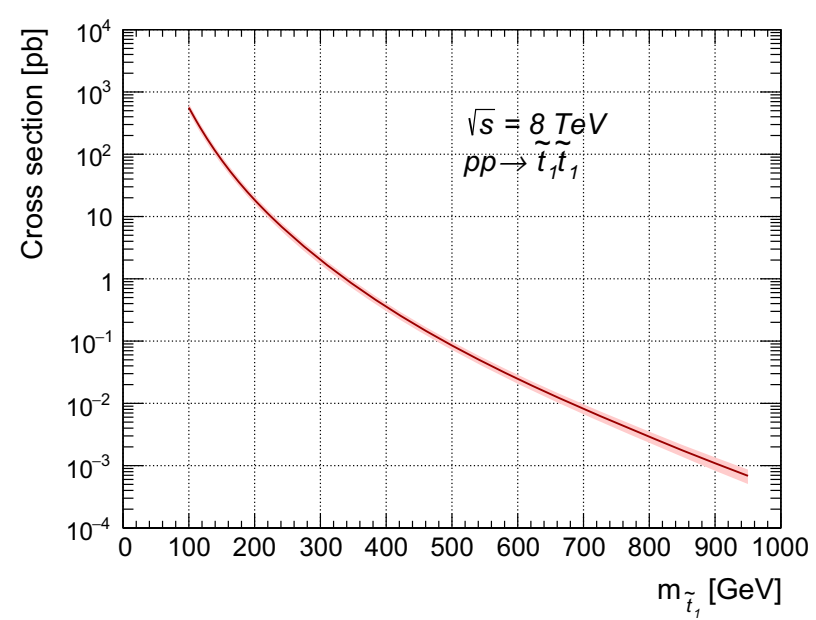

(a)

Fig. 1 a Direct stop pair production cross section at $\sqrt{s}=8 \mathrm{TeV}$ as a function of the stop mass. The band around the cross section curve illustrates the uncertainty (which is everywhere about 15-20\%) on the cross section due to scale and PDF variations. b Illustration of stop decay modes in the plane spanned by the masses of the stop $\left(\tilde{t}_{1}\right)$ and

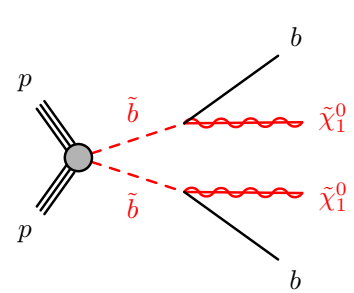

(a)

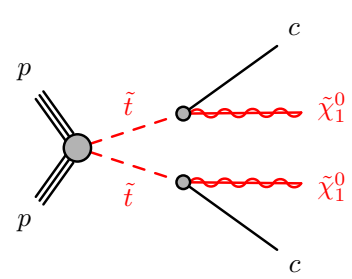

(e)

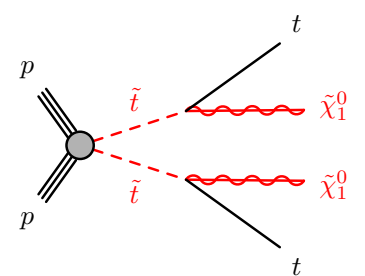

(b)

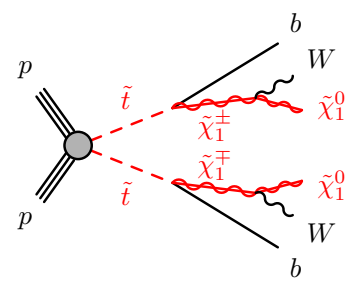

(f)
Fig. 2 Diagrams of $\tilde{t}_{1}$ and $\tilde{b}_{1}$ pair production and decays considered as simplified models: a $\tilde{b}_{1} \tilde{b}_{1} \rightarrow b \tilde{\chi}_{1}^{0} b \tilde{\chi}_{1}^{0} ; \mathbf{b} \tilde{t}_{1} \tilde{t}_{1} \rightarrow t \tilde{\chi}_{1}^{0} t \tilde{\chi}_{1}^{0} ; \mathbf{c}$ three-body decay; $\mathbf{d}$ four-body decay; e $\tilde{t}_{1} \tilde{t}_{1} \rightarrow c \tilde{\chi}_{1}^{0} c \tilde{\chi}_{1}^{0} ; \mathbf{f} \tilde{t}_{1} \tilde{t}_{1} \rightarrow b \tilde{\chi}_{1}^{ \pm} b \tilde{\chi}_{1}^{ \pm}$;

SUSY parameters arise. For example, if the lightest chargino $\left(\tilde{\chi}_{1}^{ \pm}\right)$is the next-to-lightest supersymmetric particle (NLSP), then the stop tends to have a significant branching ratio for $\tilde{t}_{1} \rightarrow b \tilde{\chi}_{1}^{ \pm}$(Fig. 2f), or, for the sbottom, $\tilde{b}_{1} \rightarrow t \tilde{\chi}_{1}^{ \pm}$ if kinematically allowed (Fig. $2 \mathrm{~g}$ ). The presence of additional particles in the decay chain makes the phenomenology depend on their masses. Several possible scenarios have been considered, the most common ones being the gauge-

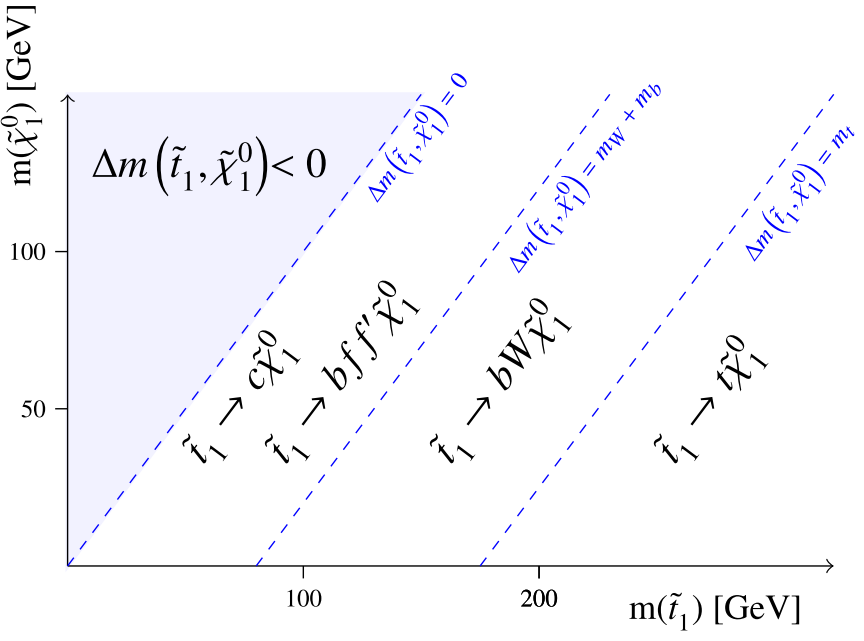

(b)

the lightest neutralino $\left(\tilde{\chi}_{1}^{0}\right)$, where the latter is assumed to be the lightest supersymmetric particle and the only one present among the decay products. The dashed blue lines indicate thresholds separating regions where different processes dominate

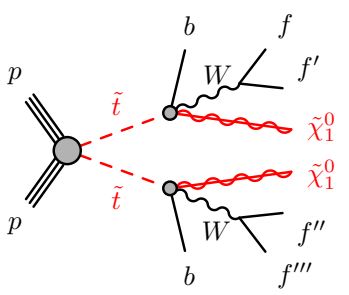

(c)

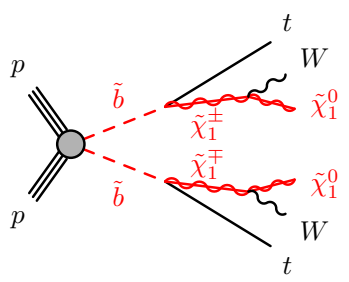

(g)

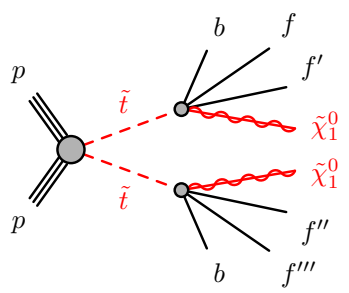

(d)

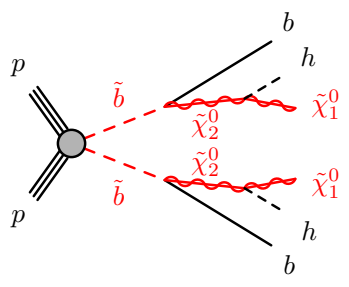

(h) g $\tilde{b}_{1} \tilde{b}_{1} \rightarrow t \tilde{\chi}_{1}^{ \pm} t \tilde{\chi}_{1}^{ \pm} ; \mathbf{h} \tilde{b}_{1} \tilde{b}_{1} \rightarrow b \tilde{\chi}_{2}^{0} b \tilde{\chi}_{2}^{0}$. The diagrams do not show "mixed" decays, in which the two pair-produced third-generation squarks decay to different final states

universality inspired $m_{\tilde{\chi}_{1}^{ \pm}}=2 m_{\tilde{\chi}_{1}^{0}}$, favoured, for example, in mSUGRA/CMSSM models [51-56]; other interpretations include the case of a chargino almost degenerate with the neutralino, a chargino almost degenerate with the squark, or a chargino of fixed mass. Another possible decay channel considered for the sbottom is $\tilde{b}_{1} \rightarrow b \tilde{\chi}_{2}^{0} \rightarrow b h \tilde{\chi}_{1}^{0}$ (Fig. 2h), which occurs in scenarios with a large higgsino component of the two lightest neutralinos. 


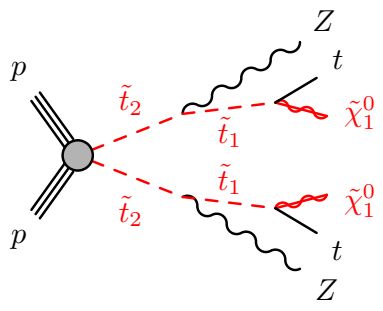

(a)

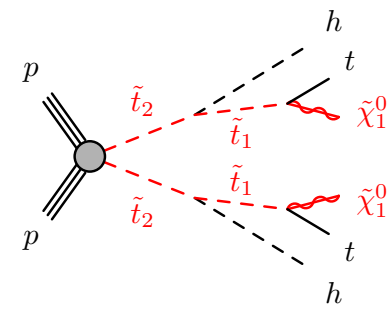

(b)

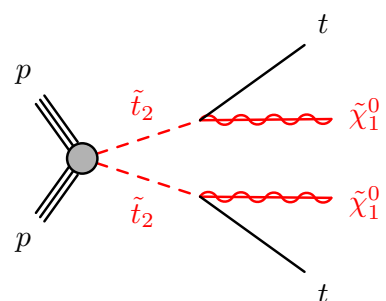

(c)
Fig. 3 Diagrams of $\tilde{t}_{2}$ decays considered as simplified models: a $\tilde{t}_{2} \tilde{t}_{2} \rightarrow \tilde{t}_{1} Z \tilde{t}_{1} Z ; \mathbf{b} \tilde{t}_{2} \tilde{t}_{2} \rightarrow \tilde{t}_{1} h \tilde{t}_{1} h ; \mathbf{c} \tilde{t}_{2} \tilde{t}_{2} \rightarrow t \tilde{\chi}_{1}^{0} t \tilde{\chi}_{1}^{0}$. The diagrams do not show "mixed" decays, in which the two pair-produced third-

Despite the lower production cross section and similar final states to $\tilde{t}_{1}$, the heavier stop state $\left(\tilde{t}_{2}\right)$ pair production has also been studied: the search for it becomes interesting in scenarios where the detection of $\tilde{t}_{1}$ pair production becomes difficult (for example if $\Delta m\left(\tilde{t}_{1}, \tilde{\chi}_{1}^{0}\right) \sim m_{t}$ ). The diagrams of the investigated processes are shown in Fig. 3.

Two types of SUSY models are used to interpret the results in terms of exclusion limits. The simplified model approach assumes that either a stop or a sbottom pair is produced and that they decay into well-defined final states, involving one or two decay channels. Simplified models are used to optimise the analyses for a specific final-state topology, rather than the complex (and model-dependent) mixture of different topologies that would arise from a SUSY model involving many possible allowed production and decay channels. The sensitivity to simplified models is discussed in Sect. 4.

More complete phenomenological minimal supersymmetric extensions of the Standard Model (pMSSM in the following [57]) are also considered, to assess the performance of the analyses in scenarios where the stop and sbottom typically have many allowed decay channels with competing branching ratios. Three different sets of pMSSM models are considered, which take into account experimental constraints from LHC direct searches, satisfying the Higgs boson mass and dark-matter relic density constraints, or additional constraints arising from considerations of naturalness. The sensitivity to these models is discussed in Sect. 5.

\section{General discussion of the analysis strategy}

The rich phenomenology of third-generation supersymmetric particles requires several event selections to target the wide range of possible topologies. A common analysis strategy and common statistical techniques, which are extensively described in Ref. [58], are employed.

Signal regions (SR) are defined, which target one specific model and SUSY particle mass range. The event selection is optimised by relying on the Monte Carlo simulation of both generation squarks decay to different final states. The decay $\tilde{t}_{2} \rightarrow \gamma \tilde{t}_{1}$ is not an allowed process

the Standard Model (SM) background production processes and the signal itself. The optimisation process aims to maximise the expected significance for discovery or exclusion for each of the models considered.

For each SR, multiple control regions (CR) are defined: they are used to constrain the normalisation of the most relevant SM production processes and to validate the MC predictions of the shapes of distributions of the kinematic variables used in the analysis. The event selection of the CRs is mutually exclusive with that of the SRs. It is, however, chosen to be as close as possible to that of the signal region while keeping the signal contamination small, and such that the event yield is dominated by one specific background process.

A likelihood function is built as the product of Poisson probability functions, describing the observed and expected number of events in the control and signal regions. The observed numbers of events in the various CRs and SRs are used in a combined profile likelihood fit [59] to determine the expected SM background yields for each of the SRs. Systematic uncertainties are treated as nuisance parameters in the fit and are constrained with Gaussian functions with standard deviation equal to their value. The fit procedure takes into account correlations in the yield predictions between different regions due to common background normalisation parameters and systematic uncertainties, as well as contamination from SUSY signal events, when a particular model is considered for exclusion.

The full procedure is validated by comparing the background predictions and the shapes of the distributions of the key analysis variables from the fit results to those observed in dedicated validation regions (VRs), which are defined to be orthogonal to, and kinematically similar, to the signal regions, with low potential contamination from signal.

After successful validation, the observed yields in the signal regions are compared to the prediction. The profile likelihood ratio statistic is used first to verify the SM backgroundonly hypothesis, and, if no significant excess is observed, to exclude the signal-plus-background hypothesis in specific signal models. A signal model is said to be excluded at $95 \%$ confidence level (CL) if the $\mathrm{CL}_{S}[60,61]$ of the profile likeli- 
hood ratio statistics of the signal-plus-background hypothesis is below 0.05 .

Several publications, targeting specific stop and sbottom final-state topologies, were published by the ATLAS collaboration at the end of the proton-proton collision run at $\sqrt{s}=8 \mathrm{TeV}$, using a total integrated luminosity of about 20 $\mathrm{fb}^{-1}$. Each of these papers defined one or more sets of signal regions optimised for different simplified models with different mass hierarchies and decay modes for the stop and/or sbottom. A few additional signal regions, focusing on regions of the parameter space not well covered by existing analyses have been defined since then. All signal regions that are used in this paper are discussed in detail in Appendix B, while Table 1 introduces their names and the targeted models. Each analysis is identified by a short acronym defined in the second column of Table 1 . The signal region names of previously published analyses are retained, but, to avoid confusion and to ease the bookkeeping, the analysis acronym is prepended to their names. For example, SRA1 from the $0 \mathrm{~L}$ analysis of Ref. [16], which is a search for stop pair production in channels with no leptons in the final state, is referred to as tOL-SRA1.

\section{Interpretations in simplified models}

The use of simplified models for analysis optimisation and result interpretation has become more and more common in the last years. The attractive feature of this approach is that it focuses on a specific final-state topology, rather than on a complex (and often heavily model-dependent) mixture of several different topologies: only a few SUSY particles are assumed to be produced in the proton-proton collision - often just one type - and only a few decay channels are assumed to be allowed. In the remainder of this section, several exclusion limits derived in different supersymmetric simplified models are presented. Details about how the MC signal samples used for the limit derivations were produced are available in Appendix D.

\subsection{Stop decays with no charginos in the decay chain}

A first series of simplified models is considered. It includes direct stop pair production as the only SUSY production process, and assumes that no supersymmetric particle other than the $\tilde{t}_{1}$ itself and the LSP, taken to be the lightest neutralino $\tilde{\chi}_{1}^{0}$, is involved in the decay. Under this assumption, there is little model dependence left in the stop phenomenology, as discussed in Sect. 2. The stop decay modes are defined mainly by the mass separation $\Delta m\left(\tilde{t}_{1}, \tilde{\chi}_{1}^{0}\right)$ between the stop and the neutralino, as shown in Fig. 1b. The corresponding diagrams are shown in Fig. 2.
Figure 4 shows the $95 \%$ CL exclusion limits obtained in the $m_{\tilde{t}_{1}}-m_{\tilde{\chi}_{1}^{0}}$ plane by the relevant analyses listed in Table 1 and discussed in Appendix B, or by their combination. A detailed discussion of which analysis is relevant in each range of $\Delta m\left(\tilde{t}_{1}, \tilde{\chi}_{1}^{0}\right)$ follows.

$\boldsymbol{\Delta} \boldsymbol{m}\left(\tilde{\boldsymbol{t}}_{\mathbf{1}}, \tilde{\chi}_{\mathbf{1}}^{\mathbf{0}}\right)<\boldsymbol{m}_{W}+\boldsymbol{m}_{\boldsymbol{b}}$ This kinematic region is characterised by the presence of two competing decays: the flavour-violating decay $\tilde{t}_{1} \rightarrow c \tilde{\chi}_{1}^{0}$ (Fig. 2e) and the fourbody decay $\tilde{t}_{1} \rightarrow b f f^{\prime} \tilde{\chi}_{1}^{0}$ (Fig. $2 \mathrm{~d}$ ). Which one of the two becomes dominant depends on the model details, in particular on the mass separation between the stop and the neutralino, and on the amount of flavour violation allowed in the model [50]. Several analyses have sensitivity in this region of the $m_{\tilde{t}_{1}}-m_{\tilde{\chi}_{1}^{0}}$ plane. The monojet-like signal regions (tc-M1$3)$ dominate the sensitivity in the region with $\Delta m\left(\tilde{t}_{1}, \tilde{\chi}_{1}^{0}\right) \gtrsim$ $m_{b}$, regardless of the decay of the stop pair, which goes undetected: their selection is based on the presence of an initial-state radiation (ISR) jet recoiling against the stop-pair system, which is assumed to be invisible. At larger values of $\Delta m\left(\tilde{t}_{1}, \tilde{\chi}_{1}^{0}\right)$, signal regions requiring the presence of a $c$-tagged jet (tc-C1-2) complement the monojet-like signal regions by targeting the $\tilde{t}_{1} \rightarrow c \tilde{\chi}_{1}^{0}$ decay. Limits on four-body decays can be set using signal regions which include low transverse momentum electrons and muons (t1L-bCa_low and WW).

The limits reported in Fig. 4 for these values of $\Delta m$ all assume that the branching ratio of the stop decay into either $\tilde{t}_{1} \rightarrow c \tilde{\chi}_{1}^{0}$ or $\tilde{t}_{1} \rightarrow b f f^{\prime} \tilde{\chi}_{1}^{0}$ is $100 \%$. However, this assumption can be relaxed, and exclusion limits derived as a function of the branching ratio of the $\tilde{t}_{1} \rightarrow c \tilde{\chi}_{1}^{0}$ decay, $\operatorname{BR}\left(\tilde{t}_{1} \rightarrow c \tilde{\chi}_{1}^{0}\right)$, assuming that $\operatorname{BR}\left(\tilde{t}_{1} \rightarrow c \tilde{\chi}_{1}^{0}\right)+\operatorname{BR}\left(\tilde{t}_{1} \rightarrow b f f^{\prime} \tilde{\chi}_{1}^{0}\right)=1$. Two different scenarios, with $\Delta m\left(\tilde{t}_{1}, \tilde{\chi}_{1}^{0}\right)=10,80 \mathrm{GeV}$, are considered. The first compressed scenario is characterised by low- $p_{\mathrm{T}}$ stop decay products, and the set of signal regions which have sensitivity is the tc- $M$, independently of the decay of the stop. In the second scenario, the phase space available for the $\tilde{t}_{1}$ decay is larger, and the full set of tc-M, tc-C, t1LbCa_low, t1L-bCa_med and WW-SR selections have different sensitivity, depending on $\operatorname{BR}\left(\tilde{t}_{1} \rightarrow c \tilde{\chi}_{1}^{0}\right)$.

The cross-section limit is derived by combining the analyses discussed above. The SR giving the lowest expected exclusion $\mathrm{CL}_{s}$ for each signal model and for each value of $\operatorname{BR}\left(\tilde{t} \rightarrow c \tilde{\chi}_{1}^{0}\right)$ is chosen.

Figure 5 shows the result of these combinations. For $\Delta m\left(\tilde{t}_{1}, \tilde{\chi}_{1}^{0}\right)=10 \mathrm{GeV}$, the sensitivity is completely dominated by the tc-M signal regions, hence no significant dependence on $\operatorname{BR}\left(\tilde{t} \rightarrow c \tilde{\chi}_{1}^{0}\right)$ is observed. In this case, stop masses up to about $250 \mathrm{GeV}$ are excluded. For $\Delta m\left(\tilde{t}_{1}, \tilde{\chi}_{1}^{0}\right)=80$ $\mathrm{GeV}$, the sensitivity is dominated by the tc-C signal regions at high values of $\operatorname{BR}\left(\tilde{t} \rightarrow c \tilde{\chi}_{1}^{0}\right)$. For lower values of $\operatorname{BR}(\tilde{t} \rightarrow$ $\left.c \tilde{\chi}_{1}^{0}\right)$, the "soft-lepton" and WW signal regions both become competitive, the latter yielding a higher sensitivity at smaller 
Table 1 Summary of the ATLAS analyses and signal regions used in this paper. Each signal region is identified by the acronym of the corresponding analysis followed by the original name of the signal region defined either in the published paper or in Appendix B.2. A dash in the signal region name column indicates that the analysis does not use the concept of signal region

\begin{tabular}{|c|c|c|c|}
\hline $\begin{array}{l}\text { Analysis name and } \\
\text { corresponding reference }\end{array}$ & Analysis acronym & Original signal region name & Model targeted \\
\hline \multirow[t]{3}{*}{ Multijet final states [16] } & \multirow[t]{3}{*}{ toL } & SRA1-4 & $\tilde{t}_{1} \rightarrow t \tilde{\chi}_{1}^{0}$ \\
\hline & & SRB & \\
\hline & & SRC1-3 & $\begin{array}{l}\tilde{t}_{1} \tilde{t}_{1} \rightarrow b t \tilde{\chi}_{1}^{0} \tilde{\chi}_{1}^{ \pm} \text {with } \\
\quad m_{\tilde{\chi}_{1}^{ \pm}}=2 m_{\tilde{\chi}_{1}^{0}}^{0}\end{array}$ \\
\hline \multirow[t]{7}{*}{ One-lepton final states [17] } & \multirow[t]{7}{*}{$\mathrm{t} 1 \mathrm{~L}$} & tN_diag & $\begin{array}{l}\tilde{t}_{1} \rightarrow t \tilde{\chi}_{1}^{0} \text { with } \\
\quad m_{\tilde{t}_{1}} \sim m_{t}+m_{\tilde{\chi}_{1}^{0}}\end{array}$ \\
\hline & & tN_med, tN_high, tN_boost & $\tilde{t}_{1} \rightarrow t \tilde{\chi}_{1}^{0}$ \\
\hline & & bCa_low, bCa_med, bCb_med1, & $\tilde{t}_{1} \rightarrow b \tilde{\chi}_{1}^{ \pm}$ \\
\hline & & bCb_high, bCb_med2, bCc_diag & \\
\hline & & bCd_bulk, bCd_high1, bCd_high2 & \\
\hline & & 3body & $\underset{\text { decay) }}{\tilde{t}_{1} \rightarrow b W \tilde{\chi}_{1}^{0} \text { (three-body }}$ \\
\hline & & tNbC_mix & $\begin{array}{l}\tilde{t}_{1} \tilde{t}_{1} \rightarrow b t \tilde{\chi}_{1}^{0} \tilde{\chi}_{1}^{ \pm} \text {with } \\
\quad m_{\tilde{\chi}_{1}^{ \pm}}=2 m_{\tilde{\chi}_{1}^{0}}\end{array}$ \\
\hline \multirow[t]{2}{*}{ Two-lepton final states [18] } & \multirow[t]{2}{*}{$\mathrm{t} 2 \mathrm{~L}$} & L90, L100, L110, L120, H160 & $\tilde{t}_{1} \rightarrow b \tilde{\chi}_{1}^{ \pm}$, three-body decay \\
\hline & & M1-4 & $\tilde{t}_{1} \rightarrow t \tilde{\chi}_{1}^{0}$ \\
\hline \multirow[t]{2}{*}{$\begin{array}{l}\text { Final states from compressed } \\
\text { stop decays [19] }\end{array}$} & \multirow[t]{2}{*}{ tc } & M1-3 & $\begin{aligned} \tilde{t}_{1} / \tilde{b}_{1} & \rightarrow \text { anything with } \\
m_{\tilde{t}_{1}} & \sim m_{\tilde{\chi}_{1}^{0}}\end{aligned}$ \\
\hline & & $\mathrm{C} 1-2$ & $\tilde{t}_{1} \rightarrow c \tilde{\chi}_{1}^{0}$ \\
\hline Final states with a $Z$ boson [20] & $\mathrm{t} 2 \mathrm{t} 1 \mathrm{Z}$ & SR2A, SR2B, SR2C, SR3A, SR3B & $\tilde{t}_{2} \rightarrow \tilde{t}_{1} Z$ and $\tilde{t}_{2} \rightarrow \tilde{t}_{1} h$ \\
\hline $\begin{array}{l}\text { Final states with two } b \text {-jets and } \\
E_{\mathrm{T}}^{\text {miss }}[21]\end{array}$ & b0L & SRA, SRB & $\begin{array}{l}\tilde{b}_{1} \rightarrow b \tilde{\chi}_{1}^{0} \text { and } \tilde{t}_{1} \rightarrow b \tilde{\chi}_{1}^{ \pm} \text {with } \\
\quad m_{\tilde{\chi}_{1}^{ \pm}} \sim m_{\tilde{\chi}_{1}^{0}}\end{array}$ \\
\hline \multirow[t]{2}{*}{$\begin{array}{l}\text { Final states with two leptons at } \\
\text { intermediate } m_{\mathrm{T} 2} \text { (Appendix } \\
\text { B.2.1) }\end{array}$} & \multirow[t]{2}{*}{ WW } & \multirow[t]{2}{*}{ SR1-7 } & $\begin{array}{l}\tilde{t}_{1} \rightarrow b \tilde{\chi}_{1}^{ \pm} \text {with } \\
\quad m_{\tilde{\chi}_{1}^{ \pm}}=m_{\tilde{t}_{1}}-10 \mathrm{GeV} \text { and }\end{array}$ \\
\hline & & & $\begin{array}{l}\tilde{t}_{1} \rightarrow b \ell v \tilde{\chi}_{1}^{0} \text { (three- and } \\
\text { four-body decays) }\end{array}$ \\
\hline $\begin{array}{l}\text { Final states containing two top } \\
\text { quarks and a Higgs } \\
\text { boson (Appendix B.2.2) }\end{array}$ & $\mathrm{t} 2 \mathrm{t} 1 \mathrm{~h}$ & - & $\tilde{t}_{2} \rightarrow \tilde{t}_{1} h$ \\
\hline $\begin{array}{l}\text { Final states containing a top } \\
\text { and a } b \text {-quark (Appendix } \\
\text { B.2.3) }\end{array}$ & tb & SR1-5 & $\begin{array}{l}\tilde{t}_{1} \tilde{t}_{1} \rightarrow b \tilde{\chi}_{1}^{ \pm} t \tilde{\chi}_{1}^{0} \text { with } \\
\quad m_{\tilde{\chi}_{1}^{ \pm}} \sim m_{\tilde{\chi}_{1}^{0}} \text { and pMSSM } \\
\text { models }\end{array}$ \\
\hline \multirow[t]{3}{*}{$\begin{array}{l}\text { Final states with three } \\
b \text {-jets [62] }\end{array}$} & \multirow[t]{3}{*}{$\mathrm{g} 3 \mathrm{~b}$} & SR- $0 \ell-4 \mathrm{j}-\mathrm{A}, \mathrm{SR}-0 \ell-4 \mathrm{j}-\mathrm{B}, \mathrm{SR}-0 \ell-4 \mathrm{j}-\mathrm{C}$ & $\begin{array}{l}\text { Gluino-mediated } \tilde{t}_{1} \text { and } \tilde{b}_{1} \\
\text { production, }\end{array}$ \\
\hline & & 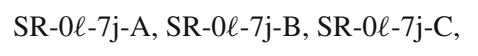 & $\tilde{b}_{1} \rightarrow \tilde{\chi}_{2}^{0} b \rightarrow \tilde{\chi}_{1}^{0} h b$ \\
\hline & & SR-1 $\ell-6 \mathrm{j}-\mathrm{A}, \mathrm{SR}-1 \ell-6 \mathrm{j}-\mathrm{B}, \mathrm{SR}-1 \ell-7 \mathrm{j}-\mathrm{C}$ & \\
\hline $\begin{array}{l}\text { Strongly produced final states } \\
\text { with two same-sign or three } \\
\text { leptons [63] }\end{array}$ & SS3L & $\begin{array}{l}\text { SR3b, SR0b, SR1b, } \\
\text { SR3Llow, S3Lhigh }\end{array}$ & $\begin{array}{l}\text { Generic gluino and squark } \\
\text { production, } \tilde{b}_{1} \rightarrow t \tilde{\chi}_{1}^{ \pm}\end{array}$ \\
\hline $\begin{array}{l}\text { Spin correlation in } t \bar{t} \\
\text { production events [64] }\end{array}$ & $\mathrm{SC}$ & - & $\begin{array}{l}\tilde{t}_{1} \rightarrow t \tilde{\chi}_{1}^{0} \text { with } \\
\quad m_{\tilde{t}_{1}} \sim m_{t}+m_{\tilde{\chi}_{1}^{0}}\end{array}$ \\
\hline$t \bar{t}$ production cross section [65] & $\mathrm{xsec}$ & - & $\tilde{t}_{1} \rightarrow t \tilde{\chi}_{1}^{0}$, three-body decay \\
\hline
\end{tabular}




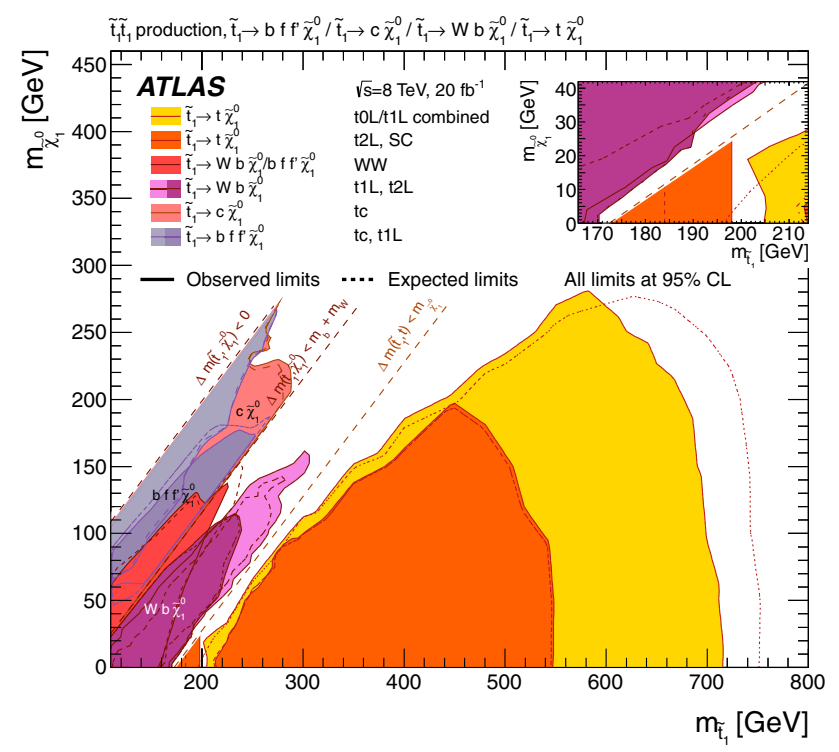

Fig. 4 Summary of the ATLAS Run 1 searches for direct stop pair production in models where no supersymmetric particle other than the $\tilde{t}_{1}$ and the $\tilde{\chi}_{1}^{0}$ is involved in the $\tilde{t}_{1}$ decay. The $95 \%$ CL exclusion limits are shown in the $m_{\tilde{t}_{1}}-m_{\tilde{\chi}_{1}^{0}}$ mass plane. The dashed and solid lines show the expected and observed limits, respectively, including all uncertainties except the theoretical signal cross-section uncertainty (PDF and scale). Four decay modes are considered separately with a branching ratio of $100 \%: \tilde{t}_{1} \rightarrow t \tilde{\chi}_{1}^{0}$, where the $\tilde{t}_{1}$ is mostly $\tilde{t}_{\mathrm{R}}$, for $\Delta m\left(\tilde{t}_{1}, \tilde{\chi}_{1}^{0}\right)>m_{t}$; $\tilde{t}_{1} \rightarrow W b \tilde{\chi}_{1}^{0}$ (three-body decay) for $m_{W}+m_{b}<\Delta m\left(\tilde{t}_{1}, \tilde{\chi}_{1}^{0}\right)<m_{t}$; $\tilde{t}_{1} \rightarrow c \tilde{\chi}_{1}^{0}$ and $\tilde{t}_{1} \rightarrow b f f^{\prime} \tilde{\chi}_{1}^{0}$ (four-body decay) for $\Delta m\left(\tilde{t}_{1}, \tilde{\chi}_{1}^{0}\right)<$ $m_{W}+m_{b}$. The latter two decay modes are superimposed

values of the stop mass. The maximum excluded stop mass ranges from about $180 \mathrm{GeV}$ for $\operatorname{BR}\left(\tilde{t} \rightarrow c \tilde{\chi}_{1}^{0}\right)=25 \%$ to about $270 \mathrm{GeV}$ for $\mathrm{BR}\left(\tilde{t} \rightarrow c \tilde{\chi}_{1}^{0}\right)=100 \%$.

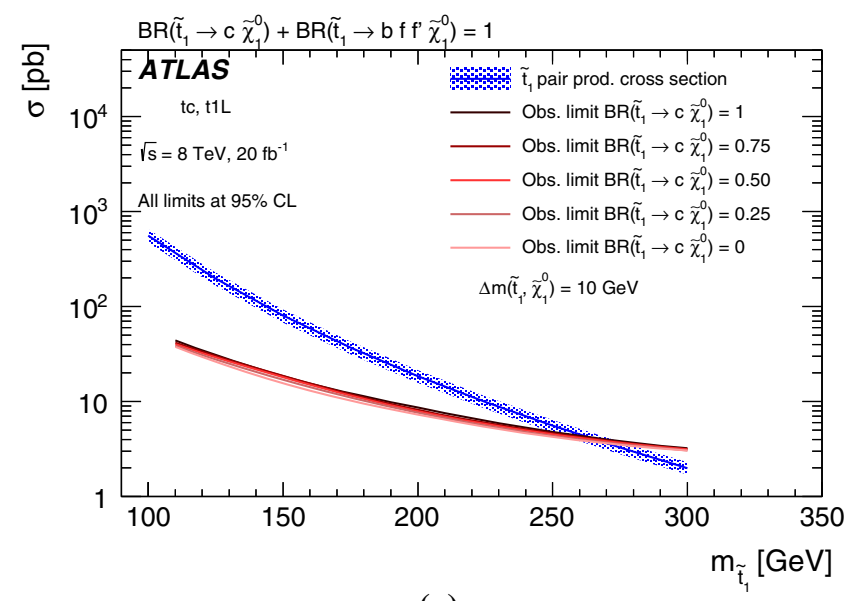

(a)

Fig. 5 Upper limits on the stop pair production cross sections for different values of the BRs for the decays $\tilde{t}_{1} \rightarrow c \tilde{\chi}_{1}^{0}$ and $\tilde{t}_{1} \rightarrow f f^{\prime} b \tilde{\chi}_{1}^{0}$. Signal points with $\Delta m\left(\tilde{t}_{1}, \tilde{\chi}_{1}^{0}\right)$ of $10 \mathrm{GeV}(\mathbf{a})$ and $80 \mathrm{GeV}(\mathbf{b})$ are shown. The limits quoted are taken from the best performing, based on expected exclusion $\mathrm{CL}_{s}$, signal regions from the tc-M, tc-C, t1L-bCa_low and
$\boldsymbol{m}_{W}+\boldsymbol{m}_{\boldsymbol{b}}<\boldsymbol{\Delta} \boldsymbol{m}\left(\tilde{\boldsymbol{t}}_{1}, \tilde{\chi}_{1}^{\mathbf{0}}\right)<\boldsymbol{m}_{t}$ In this case, the three-body decay of Fig. $2 \mathrm{c}$ is dominant. The signal regions that are sensitive to this decay are the dedicated signal region defined in the analysis selecting one-lepton final states (the t1L-3body) and the combination of several signal regions from the analysis selecting two-lepton final states, the $2 \mathrm{~L}$. The exclusion limits shown in Fig. 4 assume $\operatorname{BR}\left(\tilde{t}_{1} \rightarrow b W \tilde{\chi}_{1}^{0}\right)=1$. The WW signal regions are found to be sensitive to the kinematic region separating the three-body from the four-body stop decay region.

$\Delta \boldsymbol{m}\left(\tilde{\boldsymbol{t}}_{\mathbf{1}}, \tilde{\chi}_{\mathbf{1}}^{\mathbf{0}}\right) \sim \boldsymbol{m}_{\boldsymbol{t}}$ In this case, the neutralinos are produced with low $p_{\mathrm{T}}$, and the kinematic properties of the signal are similar to those of SM $t \bar{t}$ production. Exclusion limits in this region were obtained by two analyses performing precision SM measurements. The first one is the measurement of the $t \bar{t}$ inclusive production cross section $\sigma_{t \bar{t}}$. Limits on $\tilde{t}_{1}$ pair production were already set in Ref. [65], which measured $\sigma_{t \bar{t}}$ in the different-flavour, opposite-sign channel $e \mu$. They were derived assuming a $\tilde{t}_{1}$ decay into an on-shell top quark, $\tilde{t}_{1} \rightarrow t \tilde{\chi}_{1}^{0}$. An extension of the limits into the threebody stop decay is discussed in Appendix B.1. For a massless neutralino, the analysis excludes stop masses from about 150 $\mathrm{GeV}$ to about $m_{t}$. The limit deteriorates for higher neutralino masses, mainly because of the softer $b$-jet spectrum and the consequent loss in acceptance. The second analysis considered is that of the top quark spin correlation (SC) which considers SM $t \bar{t}$ production with decays to final states containing two leptons (electrons or muons). The shape and normalisation of the distribution of the azimuthal angle between the two leptons is sensitive to the spin of the produced particles,

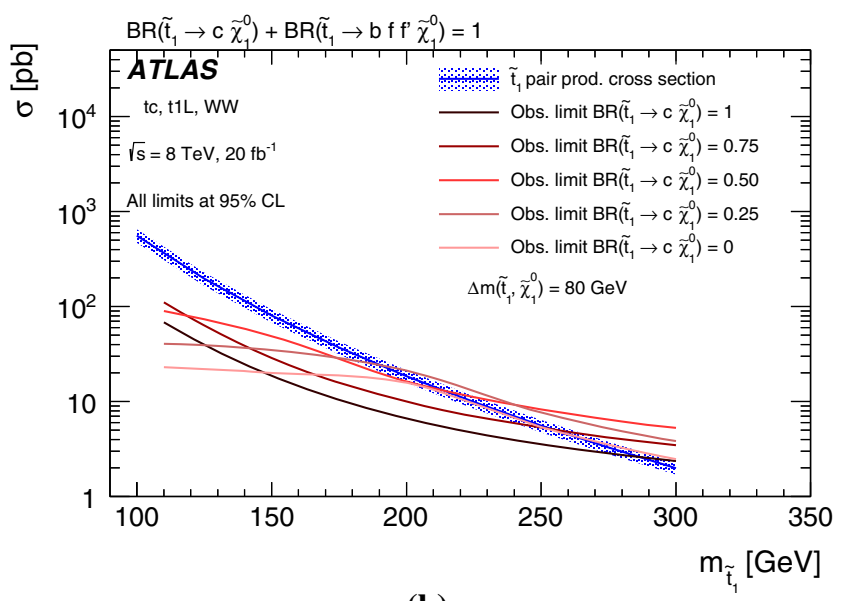

(b)

WW analyses at each mass point. The blue line and corresponding hashed band correspond to the mean value and uncertainty on the production cross section of the stop as a function of its mass. The pink lines, whose darkness indicate the value of $\operatorname{BR}\left(\tilde{t} \rightarrow c \tilde{\chi}_{1}^{0}\right)$ according to the legend, indicate the observed limit on the production cross section 
hence it allows the analysis to differentiate between stop pair and $t \bar{t}$ production. The limit obtained is shown in the bottom middle (dark orange) of the inset of Fig. 4. A small region of $\Delta m\left(\tilde{t}_{1}, \tilde{\chi}_{1}^{0}\right) \approx 180 \mathrm{GeV}$ is excluded with this measurement assuming a small neutralino mass.

$\boldsymbol{\Delta} \boldsymbol{m}\left(\tilde{\boldsymbol{t}}_{\mathbf{1}}, \tilde{\chi}_{\mathbf{1}}^{\mathbf{0}}\right)>\boldsymbol{m}_{\boldsymbol{t}}$ In this kinematic region, the decay $\tilde{t}_{1} \rightarrow$ $t \tilde{\chi}_{1}^{0}$ (see Fig. 2b) is dominant. The best results in this region are obtained by a statistical combination of the results of the multijet (t0L) and one-lepton (t1L) analyses. They both have dedicated signal regions targeting this scenario and the expected sensitivity is comparable for the two analyses. The number of required leptons makes the two signal regions mutually exclusive.

To maximise the sensitivity to the $\tilde{t}_{1} \rightarrow t \tilde{\chi}_{1}^{0}$ decays a statistical combination of the $\mathrm{t} 0 \mathrm{~L}$ and $\mathrm{t} 1 \mathrm{~L}$ signal regions is performed. The details of the combination are given in Appendix $\mathrm{C}$ and the final limit is shown in Fig. 4 by the largest shaded region (yellow). The expected limit on the stop mass is about $50 \mathrm{GeV}$ higher at low $m_{\tilde{\chi}_{1}^{0}}$ than in the individual analyses. The observed limit is increased by roughly the same amount and stop masses between 200 and $700 \mathrm{GeV}$ are excluded for small neutralino masses. ${ }^{5}$

A similar combination is performed to target a scenario where the stop can decay as $\tilde{t}_{1} \rightarrow t \tilde{\chi}_{1}^{0}$ with branching ratio $x$ and as $\tilde{t}_{1} \rightarrow b \tilde{\chi}_{1}^{ \pm}$with branching ratio $1-x$. Assuming gauge universality, the mass of the chargino is set to be twice that of the neutralino. Neutralino masses below $50 \mathrm{GeV}$ are not considered, to take into account limits on the lightest chargino mass obtained at LEP [66-70]. The exclusion limits are derived for $x=75,50,25$ and $0 \% .{ }^{6}$ Regardless of the branching ratio considered, it is always assumed that $m_{\tilde{t}_{1}}>m_{t}+m_{\tilde{\chi}_{1}^{0}}$ and $m_{\tilde{t}_{1}}>m_{b}+m_{\tilde{\chi}_{1}^{ \pm}}$, such that the two decays $\tilde{t} \rightarrow t \tilde{\chi}_{1}^{0}$ and $\tilde{t} \rightarrow b \tilde{\chi}_{1}^{ \pm}$are both kinematically allowed. A statistical combination, identical to the one described above, is used for $x=75 \%$. For smaller values of $x$, no combined fit is performed, as the sensitivity is dominated by the $1 \mathrm{~L}$ analysis almost everywhere: rather either the $\mathrm{t} 0 \mathrm{~L}$ or the $\mathrm{t} 1 \mathrm{~L}$ analysis is used, depending which one gives the smaller expected $\mathrm{CL}_{s}$ value.

Figure 6 shows the result of the combination in the $m_{\tilde{t}_{1}}-m_{\tilde{\chi}_{1}^{0}}$ plane. The limit is improved, with respect to the individual analyses, by about $50 \mathrm{GeV}$ for $m_{\tilde{\chi}_{1}^{0}}=50 \mathrm{GeV}$ and $x=75 \%$. For other $x$ values, the t1 $\mathrm{L}$ analysis is used on the full plane, with the exception of the point at the highest stop mass for $m_{\tilde{\chi}_{1}^{0}}=50 \mathrm{GeV}$ at $x=50$ and $25 \%$. Stop masses

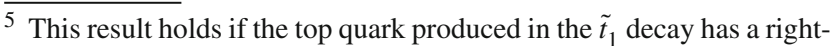
handed chirality. The dependence of the individual limits on the top quark chirality is discussed in Refs. [16,17].

${ }^{6}$ A value of $x=0 \%$ is in fact not achievable in a real supersymmetric model. Nevertheless, this value has been considered as the limiting case of a simplified model.
}

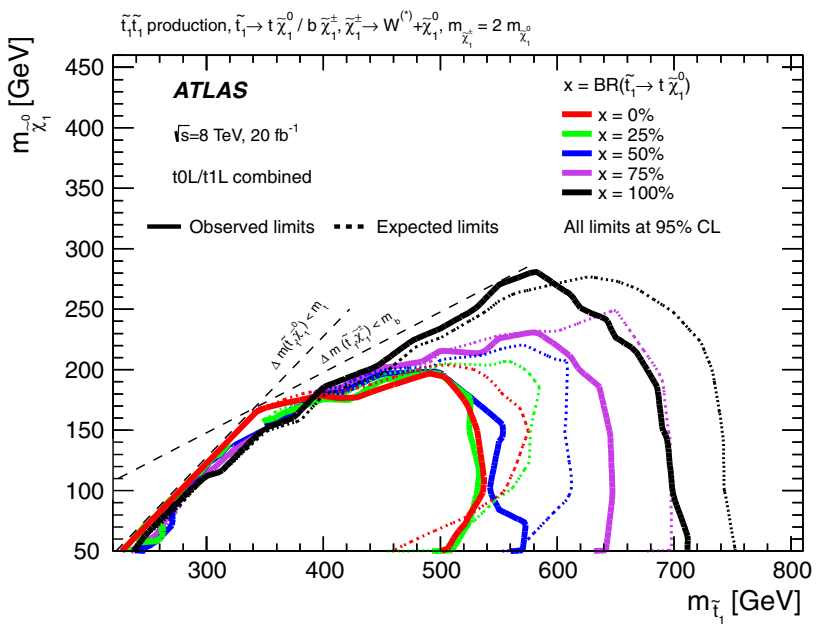

Fig. 6 Combined exclusion limits assuming that the stop decays through $\tilde{t}_{1} \rightarrow t \tilde{\chi}_{1}^{0}$ with different branching ratios $x$ and through $\tilde{t}_{1} \rightarrow b \tilde{\chi}_{1}^{ \pm}$with branching ratios $1-x$. The limits assume $m_{\tilde{\chi}_{1}^{ \pm}}=2 m_{\tilde{\chi}_{1}^{0}}$, and values of $x$ from 0 to $100 \%$ are considered. For each branching ratio, the observed (with solid lines) and expected (with dashed lines) limits are shown

below $500 \mathrm{GeV}$ are excluded for $m_{\tilde{\chi}_{1}^{0}}<160 \mathrm{GeV}$ for any value of $x$.

\subsection{Stop decays with a chargino in the decay chain}

In the pMSSM, unless the higgsino-gaugino mass parameters are related by $M_{1} \ll \mu, M_{2}$, the mass difference between the lightest neutralino and the lightest chargino cannot be too large. The mass hierarchy $m_{\tilde{\chi}_{1}^{0}}<m_{\tilde{\chi}_{1}^{ \pm}}<m_{\tilde{t}_{1}}$ is, hence, well motivated, leading to the decay chain shown in Fig. 2f.

If additional particles beside the stop and the lightest neutralino take part in the stop decay, the stop phenomenology quickly becomes complex. Even if the chargino is the only other relevant SUSY particle, the stop phenomenology depends on the chargino mass, on the stop left-right mixing, and on the composition of the neutralino and chargino in terms of bino, wino and higgsino states.

Figure 7 shows the exclusion limits obtained by the analyses listed in Table 1 and discussed in Appendix B if a branching ratio of $100 \%$ for $\tilde{t} \rightarrow b \tilde{\chi}_{1}^{ \pm}$is assumed. The exclusion limits are presented in a number of $m_{\tilde{t}_{1}}-m_{\tilde{\chi}_{1}^{0}}$ planes, each characterised by a different hypothesis on the chargino mass. For all scenarios considered, the chargino is assumed to decay as $\tilde{\chi}_{1}^{ \pm} \rightarrow W^{(*)} \tilde{\chi}_{1}^{0}$, where the $(*)$ indicates a possibly virtual $W$ boson.

$\Delta m\left(\tilde{\chi}_{1}^{ \pm}, \tilde{\chi}_{1}^{0}\right)=5,20 \mathrm{GeV}$ This scenario assumes that the difference in mass between the lightest chargino and the neutralino is small (Fig. 7a), which is a rather common feature of models where, for example, the LSP has a large wino or higgsino component. Two hypotheses have been considered, 


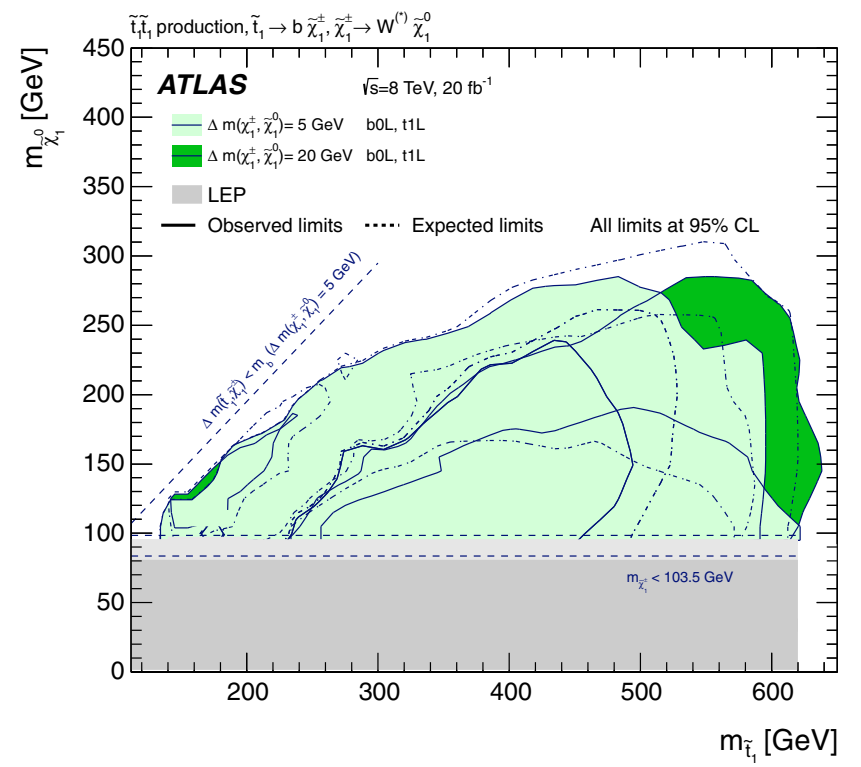

(a)

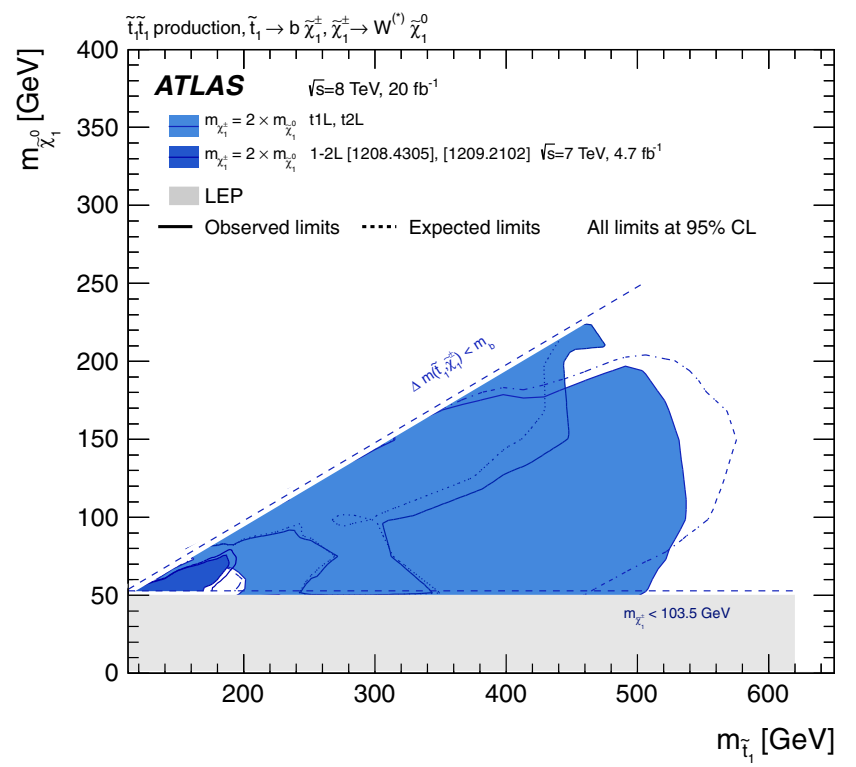

(c)

Fig. 7 Summary of the ATLAS Run 1 searches for direct stop pair production in models where the decay mode $\tilde{t}_{1} \rightarrow b \tilde{\chi}_{1}^{ \pm}$with $\tilde{\chi}_{1}^{ \pm} \rightarrow W^{*} \tilde{\chi}_{1}^{0}$ is assumed with a branching ratio of $100 \%$. Various hypotheses on the $\tilde{t}_{1}, \tilde{\chi}_{1}^{ \pm}$, and $\tilde{\chi}_{1}^{0}$ mass hierarchy are used. Exclusion limits at $95 \% \mathrm{CL}$ are shown in the $\tilde{t}_{1}-\tilde{\chi}_{1}^{0}$ mass plane. The dashed and solid lines show the expected and observed limits, respectively, including all uncertainties except the theoretical signal cross-section uncertainty (PDF and scale). Wherever not superseded by any $\sqrt{s}=8 \mathrm{TeV}$ analysis, results obtained by analyses using $4.7 \mathrm{fb}^{-1}$ of proton-proton collision data

with $\Delta m\left(\tilde{\chi}_{1}^{ \pm}, \tilde{\chi}_{1}^{0}\right)=5 \mathrm{GeV}$ and $\Delta m\left(\tilde{\chi}_{1}^{ \pm}, \tilde{\chi}_{1}^{0}\right)=20 \mathrm{GeV}$. For both, the complete decay chain is $\tilde{t}_{1} \rightarrow b \tilde{\chi}_{1}^{ \pm} \rightarrow b f f^{\prime} \tilde{\chi}_{1}^{0}$, where the transverse momenta of the fermions $f$ and $f^{\prime}$ depend on $\Delta m\left(\tilde{\chi}_{1}^{ \pm}, \tilde{\chi}_{1}^{0}\right)$ and on the stop mass, given the

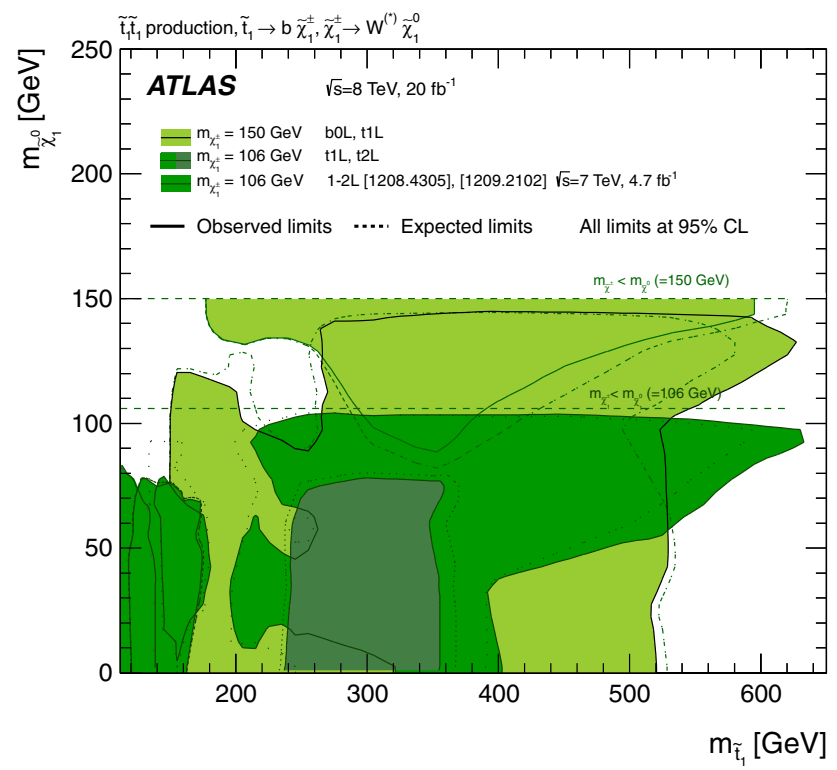

(b)

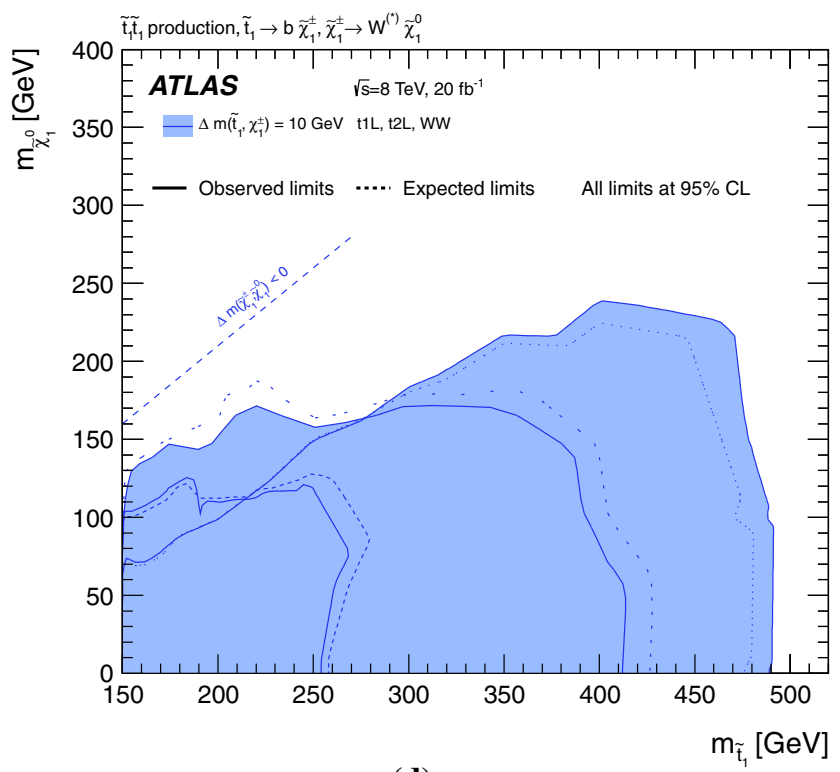

(d)

taken at $\sqrt{s}=7 \mathrm{TeV}$ are also shown, with the corresponding reference. The four plots correspond to interpretations of a the b0L and t1L softlepton analyses in two scenarios $\left(\Delta m\left(\tilde{\chi}_{1}^{ \pm}, \tilde{\chi}_{1}^{0}\right)=5 \mathrm{GeV}\right.$ in light green and $\Delta m\left(\tilde{\chi}_{1}^{ \pm}, \tilde{\chi}_{1}^{0}\right)=20 \mathrm{GeV}$ in dark green $)$, for a total of four limits; b the b0L, t1L and $2 \mathrm{~L}$ analyses in scenarios with a fixed chargino mass $m_{\tilde{\chi}_{1}^{ \pm}}=106 \mathrm{GeV}$ (dark green) and $m_{\tilde{\chi}_{1}^{ \pm}}=150 \mathrm{GeV}$ (light green); $\mathbf{c}$ the $11 \mathrm{~L}$ and $\mathrm{t} 2 \mathrm{~L}$ analyses in scenarios with $m_{\tilde{\chi}_{1}^{ \pm}}=2 m_{\tilde{\chi}_{1}^{0}}$; d interpretations of the $11 \mathrm{~L}, \mathrm{t} 2 \mathrm{~L}$ and $\mathrm{WW}$ analyses in senarios with $\Delta m\left(\tilde{t}_{1}, \tilde{\chi}_{1}^{ \pm}\right)=10 \mathrm{GeV}$

dependency on the chargino boost. If $\Delta m\left(\tilde{\chi}_{1}^{ \pm}, \tilde{\chi}_{1}^{0}\right)=5 \mathrm{GeV}$, the fermions have momenta too low to be efficiently reconstructed. The observed final state then consists of two $b$-jets and $E_{\mathrm{T}}^{\text {miss }}$. This final state is the direct target of the b0L signal 
regions. For $\Delta m\left(\tilde{\chi}_{1}^{ \pm}, \tilde{\chi}_{1}^{0}\right)=20 \mathrm{GeV}$, the signal efficiencies of the bOL signal regions decrease because of the lepton and jet veto applied. The t1L signal regions with soft leptons, instead, gain in sensitivity, profiting from the higher transverse momentum of the fermions from the off-shell $W$ decay produced in the chargino decay.

$\boldsymbol{m}_{\tilde{\chi}_{1}^{ \pm}}=106,150 \mathrm{GeV}$ This scenario (Fig. 7b) assumes a fixed chargino mass. The SR yielding the lowest expected exclusion $\mathrm{CL}_{s}$ for this scenario depends on the value of $\Delta m\left(\tilde{\chi}_{1}^{ \pm}, \tilde{\chi}_{1}^{0}\right)$. For $\Delta m\left(\tilde{\chi}_{1}^{ \pm}, \tilde{\chi}_{1}^{0}\right)<20 \mathrm{GeV}$, the b0L signal regions provide the best sensitivity; for larger values of $\Delta m\left(\tilde{\chi}_{1}^{ \pm}, \tilde{\chi}_{1}^{0}\right)$, the $\mathrm{t} 1 \mathrm{~L}$ and $\mathrm{t} 2 \mathrm{~L}$ signal regions provide better sensitivity because of the same mechanism as in the $\Delta m\left(\tilde{\chi}_{1}^{ \pm}, \tilde{\chi}_{1}^{0}\right)=5,20 \mathrm{GeV}$ scenario above. The exclusion extends up to about $600 \mathrm{GeV}$ for small values of $\Delta m\left(\tilde{\chi}_{1}^{ \pm}, \tilde{\chi}_{1}^{0}\right)$. A region of the parameter space with $m_{\tilde{t}_{1}}$ up to about $260 \mathrm{GeV}$ and $m_{\tilde{\chi}_{1}^{0}}$ between $100 \mathrm{GeV}$ and $m_{\tilde{\chi}_{1}^{ \pm}}$is not yet excluded.

$\boldsymbol{m}_{\tilde{\chi}_{1}^{ \pm}}=\mathbf{2} \boldsymbol{m}_{\tilde{\chi}_{1}^{0}}$ Inspired by gauge-universality considerations, the third scenario (Fig. 7c) is characterised by a relatively large $\Delta m\left(\tilde{\chi}_{1}^{ \pm}, \tilde{\chi}_{1}^{0}\right)$. The $\mathrm{t} 2 \mathrm{~L}$ signal regions dominate the sensitivity for $m_{\tilde{t}_{1}} \sim m_{\tilde{\chi}_{1}^{ \pm}}$. The sensitivity of the dedicated t1L$\mathrm{bC}$ is dominant in a large region of the plane, and determines the exclusion reach for moderate to large values of $\Delta m\left(\tilde{t}_{1}, \tilde{\chi}_{1}^{0}\right)$.

$\Delta \boldsymbol{m}\left(\tilde{\boldsymbol{t}}_{1}, \tilde{\chi}_{1}^{ \pm}\right)=\mathbf{1 0 ~ G e V}$ The fourth scenario (Fig. 7d) assumes a rather compressed $\tilde{t}_{1}-\tilde{\chi}_{1}^{ \pm}$spectrum. The region at low $m_{\tilde{t}_{1}}$ and large $m_{\tilde{\chi}_{1}^{0}}$ is characterised by low mass separations between all particles involved, and it is best covered by the t1L-bCc_diag, the t1L soft lepton, and the WW signal regions. At larger values of the stop mass, the leptons emitted in the $\tilde{\chi}_{1}^{ \pm}$decay have larger $p_{\mathrm{T}}$, and the $\mathrm{t} 2 \mathrm{~L}$ signal regions provide the best sensitivity.

$\boldsymbol{m}_{\tilde{t}_{1}}=300 \mathrm{GeV}$ The final scenario considered is one where the stop mass is fixed at $300 \mathrm{GeV}$, and the exclusion limits are expressed in the $m_{\tilde{\chi}_{1}^{ \pm}}-m_{\tilde{\chi}_{1}^{0}}$ plane. In the case of the compressed scenario, corresponding to a small mass difference $\Delta m\left(\tilde{\chi}_{1}^{ \pm}, \tilde{\chi}_{1}^{0}\right)$, the fermions from the $W^{(*)}$ decay can escape detection and only the two $b$-jets and $E_{\mathrm{T}}^{\text {miss }}$ would be identified in the final state. Thus, the b0L signal regions are expected to have a large sensitivity in this case, while for larger values of $\Delta m\left(\tilde{\chi}_{1}^{ \pm}, \tilde{\chi}_{1}^{0}\right)$, the lepton can be observed, yielding a final-state signature investigated by the t1L softlepton signal region. A combination of the $\mathrm{b} 0 \mathrm{~L}$ and $\mathrm{t} 1 \mathrm{~L}$ signal regions is performed by choosing, for each point of the plane, the SR giving the lowest $\mathrm{CL}_{s}$ for expected exclusion. The result, reported in Fig. 8, shows that a large portion of the plane is excluded, with the exception of a region where the mass separations between the $\tilde{t}_{1}$, the $\tilde{\chi}_{1}^{ \pm}$and the $\tilde{\chi}_{1}^{0}$ are small.

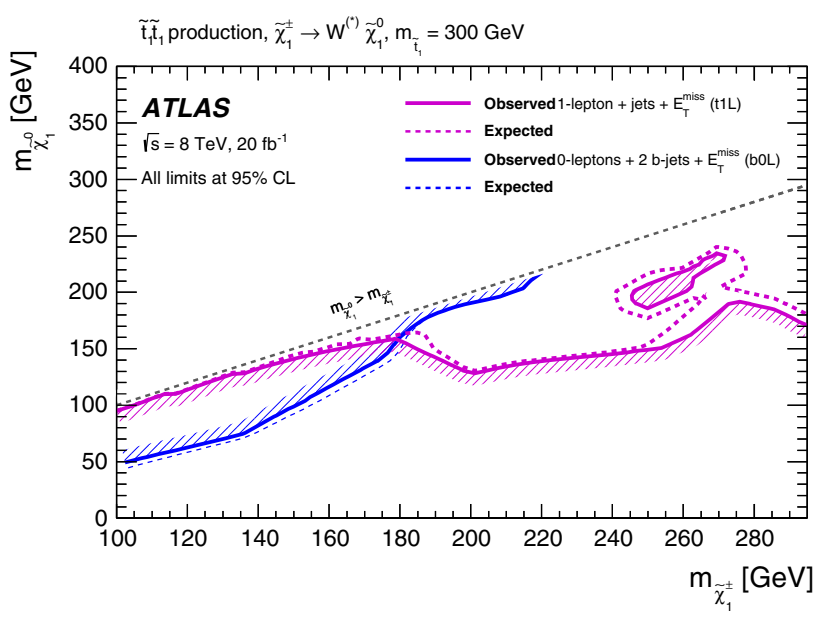

Fig. 8 Exclusion limits assuming that the stop decays through $\tilde{t}_{1} \rightarrow$ $b+\tilde{\chi}_{1}^{ \pm} \rightarrow b+W^{(*)}+\tilde{\chi}_{1}^{0}$ with branching ratio of $100 \%$ assuming a fixed stop mass of $m_{\tilde{t}_{1}}=300 \mathrm{GeV}$. The region below the purple line and above the blue line, indicated by a light shading, is excluded

Summarising, in the simplified models with $\tilde{t}_{1} \rightarrow b \tilde{\chi}_{1}^{ \pm} \rightarrow$ $b W^{(*)} \tilde{\chi}_{1}^{0}$, stop masses up to $450-600 \mathrm{GeV}$ are generally excluded. Scenarios where $\Delta m\left(\tilde{t}_{1}, \tilde{\chi}_{1}^{0}\right)$ is small are particularly difficult to exclude and in these compressed scenarios, stop masses as low as $200 \mathrm{GeV}$ are still allowed (Fig. 7b). A small unexcluded area is also left for a small region around $\left(m_{\tilde{t}_{1}}, m_{\tilde{\chi}_{1}^{ \pm}}, m_{\tilde{\chi}_{1}^{0}}\right)=(180,100,50) \mathrm{GeV}$ (Fig. 7c), where the sensitivity of the analyses is poor because the signal kinematics are similar to SM $t \bar{t}$ production.

\subsection{Limits on pair production of $\tilde{t}_{2}$}

Although the pair production of $\tilde{t}_{1}$ has a cross section larger than that of $\tilde{t}_{2}$, and although the decay patterns of the two particles can be similar, it can be convenient to search for the latter in regions where the sensitivity to the former is limited. This is the case, for example, in the region where $\Delta m\left(\tilde{t}_{1}, \tilde{\chi}_{1}^{0}\right) \sim m_{t}$ of Fig. 4 , where the separation of $\tilde{t}_{1}$ pair production from SM top quark pair production is difficult. The $\mathrm{t} 2 \mathrm{t} 1 \mathrm{Z}$ and $\mathrm{t} 2 \mathrm{t} 1 \mathrm{~h}$ analyses are designed to detect $\tilde{t}_{2}$ pair production in this region of the $m_{\tilde{t}_{1}}-m_{\tilde{\chi}_{1}^{0}}$ plane, followed by the decays $\tilde{t}_{2} \rightarrow \tilde{t}_{1} Z$ and $\tilde{t}_{2} \rightarrow \tilde{t}_{1} h$. The Higgs boson $h$ is assumed to have a mass of $125 \mathrm{GeV}$ and $\mathrm{SM}$ branching ratios.

The exclusion limits were first derived in a scenario in which the pair-produced $\tilde{t}_{2}$ decays either through $\tilde{t}_{2} \rightarrow Z \tilde{t}_{1}$ with a branching ratio of $100 \%$ (Fig. 3a), or through $\tilde{t}_{2} \rightarrow h \tilde{t}_{1}$ (again with a branching ratio of $100 \%$; Fig. $3 \mathrm{~b}$ ). In both cases, the $\tilde{t}_{1}$ is assumed to decay through $\tilde{t}_{1} \rightarrow t \tilde{\chi}_{1}^{0}$, and its mass is set to be $180 \mathrm{GeV}$ above that of the neutralino (assumed to be the LSP), which is the region not excluded in Fig. 4. The final state contains two top quarks, two neutralinos, and either two $Z$ or two $h$ bosons. 


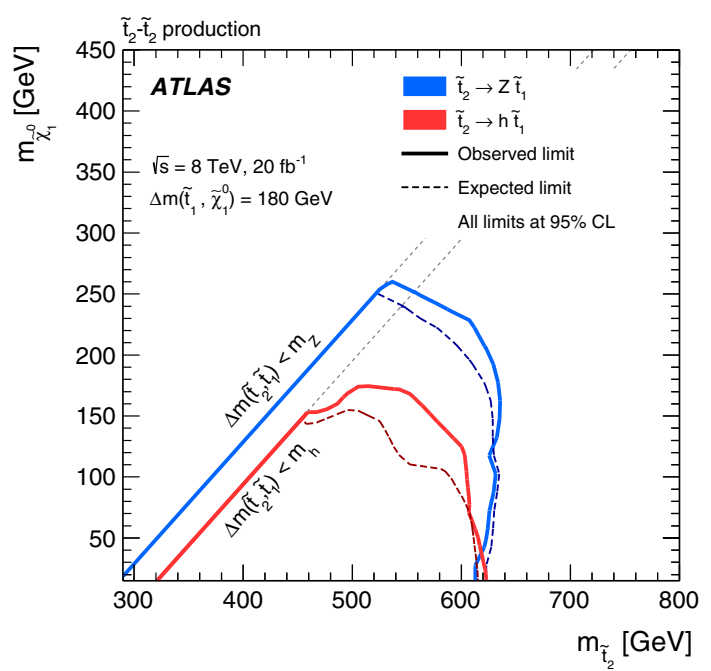

Fig. 9 Exclusion limits at $95 \% \mathrm{CL}$ in the scenario where $\tilde{t}_{2}$ pair production is assumed, followed by the decay $\tilde{t}_{2} \rightarrow Z \tilde{t}_{1}$ (blue) or $\tilde{t}_{2} \rightarrow \tilde{t}_{1} h$ (red) and then by $\tilde{t}_{1} \rightarrow t \tilde{\chi}_{1}^{0}$ with a branching ratio of $100 \%$, as a function of the $\tilde{t}_{2}$ and $\tilde{\chi}_{1}^{0}$ mass. The $\tilde{t}_{1}$ mass is determined by the relation $m_{\tilde{t}_{1}}-m_{\tilde{\chi}_{1}^{0}}=180 \mathrm{GeV}$. The dashed lines indicate the expected limit and the solid lines indicate the observed limit

Figure 9 shows the exclusion limits for the $2 \mathrm{t} 1 \mathrm{~h}$ and the $\mathrm{t} 2 \mathrm{t} 1 \mathrm{Z}$ analyses. In both cases, a limit on $m_{\tilde{t}_{2}}$ is set at about $600 \mathrm{GeV}$ for a massless neutralino. In the case of a $\tilde{t}_{2}$ decay through a Higgs boson, the limit covers neutralino masses lower than in the case of the decay through a $Z$ boson.

The assumption on the branching ratio of the $\tilde{t}_{2}$ has also been relaxed, and limits have been derived assuming that the three decays $\tilde{t}_{2} \rightarrow Z \tilde{t}_{1}, \tilde{t}_{2} \rightarrow h \tilde{t}_{1}$ and $\tilde{t}_{2} \rightarrow t \tilde{\chi}_{1}^{0}$ (Fig. 3c) are the only possible ones. The limits are shown in Fig. 10 as a function of the three BRs, for different combinations of the $\tilde{t}_{2}$ and $\tilde{\chi}_{1}^{0}$ masses. Three analyses have been considered: the $\mathrm{t} 2 \mathrm{t} 1 \mathrm{Z}, \mathrm{t} 2 \mathrm{t} 1 \mathrm{~h}$ and the combination of the $\mathrm{t} 0 \mathrm{~L}$ and $\mathrm{t} 1 \mathrm{~L}$ discussed in Sect. 4.1. ${ }^{7}$ The three analyses have complementary sensitivities. Together, they exclude $\tilde{t}_{2}$ pair production with a mass of 350 and $500 \mathrm{GeV}$ for $m_{\tilde{\chi}_{1}^{0}}=20 \mathrm{GeV}$. A nonexcluded region appears for $m_{\tilde{t}_{2}}=500 \mathrm{GeV}$ if larger $\tilde{\chi}_{1}^{0}$ masses are considered.

\subsection{Sbottom decays}

Under the assumption that no supersymmetric particle takes part in the sbottom decay apart from the lightest neutralino, the sbottom decays as $\tilde{b}_{1} \rightarrow b \tilde{\chi}_{1}^{0}$ with a branching ratio of $100 \%$ (Fig. 2a). The final state arising from sbottom pair production hence contains two $b$-jets and $E_{\mathrm{T}}^{\text {miss }}$. The b0L signal

\footnotetext{
${ }^{7}$ For the combination of the t $0 \mathrm{~L}$ and $\mathrm{t} 1 \mathrm{~L}$ analyses, the limits extracted for the $\tilde{t}_{1} \rightarrow t \tilde{\chi}_{1}^{0}$ decay with branching ratio of $100 \%$ have simply been rescaled by appropriate factors depending on the branching ratio of $\tilde{t}_{2} \rightarrow t \tilde{\chi}_{1}^{0}$ considered here.
}

regions were explicitly optimised to be sensitive to this scenario. In case of a mass degeneracy between the sbottom and the neutralino, the general consideration that the monojet-like tc-M selection is almost insensitive to the details of the decay of the produced particles still holds: the tc-M signal regions offer the best sensitivity for scenarios where $m_{\tilde{b}_{1}} \sim m_{\tilde{\chi}_{1}^{0}}$.

Figure 11 shows the limits of the tc and b0L analyses on the $m_{\tilde{b}_{1}}-m_{\tilde{\chi}_{1}^{0}}$ plane. The monojet-like (tc-M) SRs exclude models up to a value of $m_{\tilde{b}_{1}} \sim m_{\tilde{\chi}_{1}^{0}} \sim 280 \mathrm{GeV}$. Sbottom masses are excluded up to about $600 \mathrm{GeV}$ for neutralino masses below about $250 \mathrm{GeV}$.

If other supersymmetric particles enter into the decay chain, then multiple decay channels would be allowed. Similarly to the stop, the case in which other neutralinos or charginos have a mass below the sbottom is well motivated. The branching ratios of the sbottom to the different decay channels depend on the supersymmetric particle mass hierarchy, on the mixing of the left-right components of the sbottom, and on the composition of the charginos and neutralinos in terms of bino, wino, and higgsino states.

An exclusion limit is derived under the assumption that the sbottom decays with a branching ratio of $100 \%$ into $\tilde{b}_{1} \rightarrow t \tilde{\chi}_{1}^{ \pm}$(Fig. $2 \mathrm{~g}$ ). The chargino is assumed to decay through $\tilde{\chi}_{1}^{ \pm} \rightarrow W^{(*)} \tilde{\chi}_{1}^{0}$ with a branching ratio of $100 \%$. The final state is a complex one, and offers many handles for background rejection: it potentially contains up to ten jets, two $b$-jets, and up to four leptons. The limits of Fig. 12a, shown in the $m_{\tilde{b}_{1}}-m_{\tilde{\chi}_{1}^{0}}$ plane, were obtained by using the three-lepton signal regions SS3L, either fixing the mass of the neutralino to $m_{\tilde{\chi}_{1}^{0}}=60 \mathrm{GeV}$ or by making the assumption that $m_{\tilde{\chi}_{1}^{ \pm}}=2 m_{\tilde{\chi}_{1}^{0}}$. In the two scenarios considered, sbottom masses up to about $440 \mathrm{GeV}$ are excluded, with a mild dependency on the neutralino mass.

The last case considered is one where the pair-produced sbottoms decay through $\tilde{b}_{1} \rightarrow b \tilde{\chi}_{2}^{0}$, followed by the decay of $\tilde{\chi}_{2}^{0}$ into a $\tilde{\chi}_{1}^{0}$ and a SM-like Higgs boson $h$ (Fig. 2h). The final state contains up to six $b$-jets, four of which are produced by the two Higgs bosons decays. Since multiple $b$-jets are present in the final state, the three- $b$-jets signal regions ( $\mathrm{g} 3 \mathrm{~b}$ ) are used to place limits in this model.

The limit, derived as a function of $m_{\tilde{b}_{1}}$ and $m_{\tilde{\chi}_{2}^{0}}$ assuming a fixed neutralino mass of $\tilde{\chi}_{1}^{0}=60 \mathrm{GeV}$, is shown in Fig. 12b. Sbottom masses between about 300 and $650 \mathrm{GeV}$ are excluded for $\tilde{\chi}_{2}^{0}$ masses above $250 \mathrm{GeV}$.

\section{Interpretations in pMSSM models}

The interpretation of the results in simplified models is useful to assess the sensitivity of each signal region to a specific topology. However, this approach fails to test signal regions on the complexity of the stop and sbottom phenomenology 
Fig. 10 Exclusion limits as a function of the $\tilde{t}_{2}$ branching ratio for $\tilde{t}_{2} \rightarrow \tilde{t}_{1} h, \tilde{t}_{2} \rightarrow \tilde{t}_{1} Z$ and $\tilde{t}_{2} \rightarrow t \tilde{\chi}_{1}^{0}$. The blue, red and green limit refers to the $\mathrm{t} 2 \mathrm{t} 1 \mathrm{Z}$, $\mathrm{t} 2 \mathrm{t} 1 \mathrm{~h}$ and combination of $\mathrm{t} 0 \mathrm{~L}$ and $\mathrm{t} 1 \mathrm{~L}$ analyses respectively. The limits are given for three different values of the $\tilde{t}_{2}$ and $\tilde{\chi}_{1}^{0}$ masses

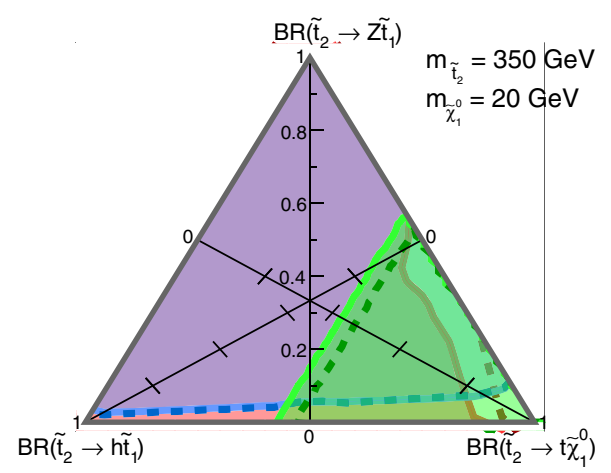

ATLAS $\sqrt{\mathrm{s}}=8 \mathrm{TeV}, 20 \mathrm{fb}^{-1}$

$\tilde{\mathrm{t}}_{2}-\tilde{\mathrm{t}}_{2}$ production, $\tilde{\mathrm{t}}_{2} \rightarrow Z \tilde{\mathrm{t}}_{1}, \tilde{\mathrm{t}}_{1}, \tilde{\mathrm{t}}_{1}^{0}$ $\Delta \mathrm{m}\left(\tilde{\mathrm{t}}_{1}, \tilde{\chi}_{1}^{0}\right)=180 \mathrm{GeV}, \tilde{\mathrm{t}}_{1} \rightarrow \mathrm{t} \tilde{\chi}_{1}^{0}$

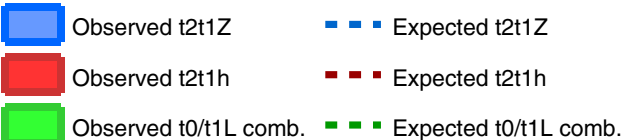
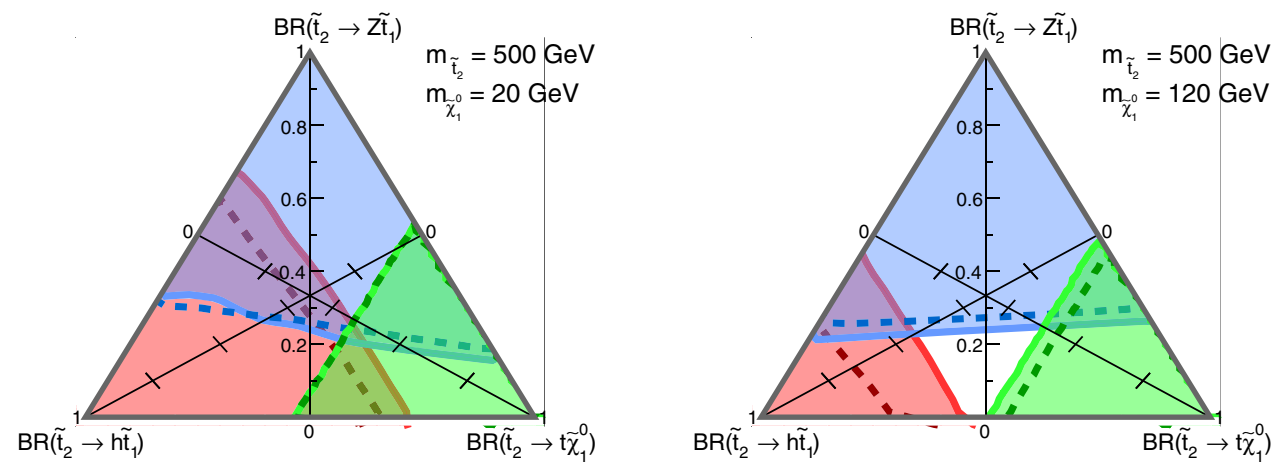

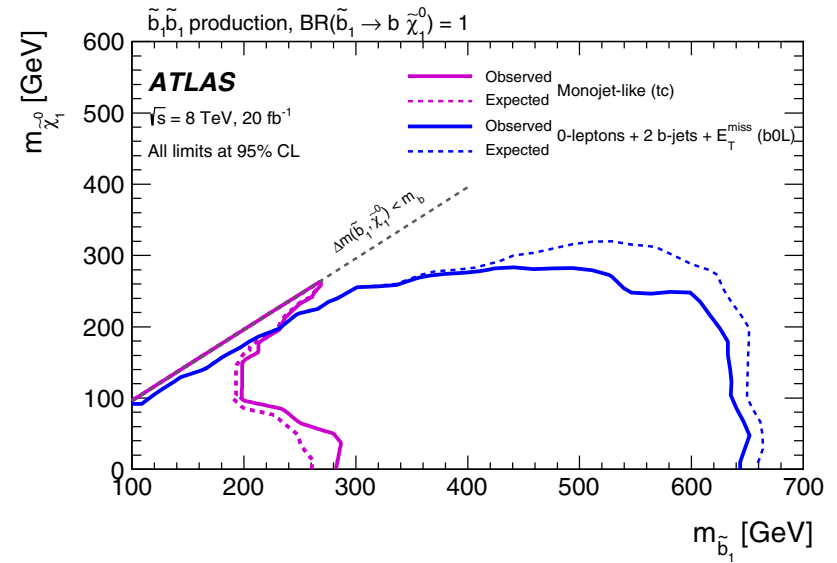

Fig. 11 Observed (solid lines) and expected (dashed lines) $95 \% \mathrm{CL}$ limits on sbottom pair production where the sbottom is assumed to decay as $\tilde{b}_{1} \rightarrow b \tilde{\chi}_{1}^{0}$ with a branching ratio of $100 \%$. The purple lines refer to the limit of the tc analysis, while the blue lines refer to the b0L analysis

that appears in a realistic SUSY model. To this extent, the signal regions are used to derive exclusion limits in the context of specific pMSSM models.

The pMSSM [57] is obtained from the more general MSSM by making assumptions based on experimental results:
- No new source of CP violation beyond the Standard Model. New sources of $\mathrm{CP}$ violation are constrained by experimental limits on the electron and neutron electric dipole moments.

- No flavour-changing neutral currents. This is implemented by requiring that the matrices for the sfermion masses and trilinear couplings are diagonal.

- First- and second-generation universality. The soft-SUSYbreaking mass parameters and the trilinear couplings for the first and second generation are assumed to be the same based on experimental data from, e.g., the neutral kaon system [71].

With the above assumptions, and with the choice of a neutralino as the LSP, the pMSSM adds 19 free parameters on top of those of the SM. The complete set of pMSSM parameters is shown in Table 2.

A full assessment of the ATLAS sensitivity to a scan of the 19-parameters space has been performed in Ref. [72]. Here, a set of additional hypotheses are made, to focus on the sensitivity to a specific, well-motivated set of models with enhanced third generation squark production:

- The common masses of the first- and second-generation squarks have been set to a multi-TeV scale, making these quarks irrelevant for the processes studied at the energies investigated in this paper. This choice is motivated by the 


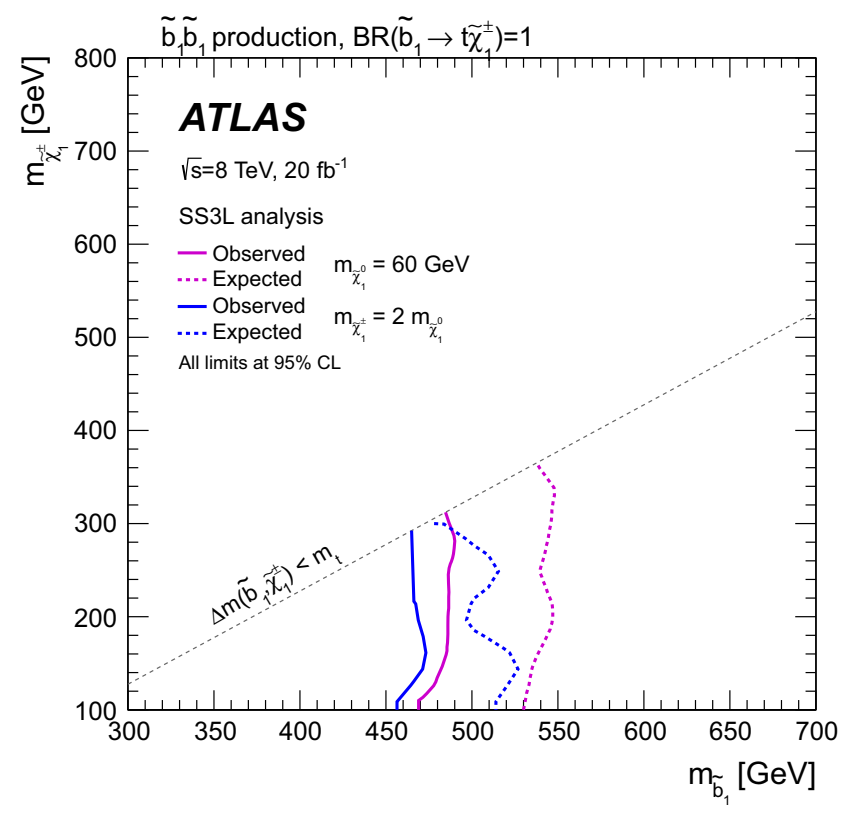

(a)

Fig. 12 Exclusion limits at $95 \% \mathrm{CL}$ for a scenario where sbottoms are pair produced and decay as $\mathbf{a} \tilde{b}_{1} \rightarrow t \tilde{\chi}_{1}^{ \pm}$with a BR of $100 \%$ or $\mathbf{b}$ $\tilde{b}_{1} \rightarrow b \tilde{\chi}_{2}^{0}$ with a BR of $100 \%$. The signal regions used in a are the SS3L, and two different models are considered: a fixed neutralino mass

Table 2 Description of the 19 additional parameters of the pMSSM model with a neutralino LSP

\begin{tabular}{ll}
\hline Parameter & Description \\
\hline$m_{\tilde{u} R}, m_{\tilde{d} R}, m_{\tilde{q} L 1}, m_{\tilde{e} R}, m_{\tilde{\ell} L 1}$ & $\begin{array}{c}\text { First- and second-generation common } \\
\text { mass parameters }\end{array}$ \\
$m_{\tilde{b} R}, m_{\tilde{t} R}, m_{\tilde{q} L 3}, m_{\tilde{\tau} R}, m_{\tilde{\ell} L 3}$ & Third-generation mass parameters \\
$M_{1}, M_{2}, M_{3}$ & Gaugino mass parameters \\
$A_{b}, A_{\tau}, A_{t}$ & Trilinear couplings \\
$\mu, M_{A}$ & Higgs/higgsino mass parameters \\
$\tan \beta$ & Ratio of vacuum expectation values of \\
& the two Higgs doublets \\
\hline
\end{tabular}

absence of any signal from squark or gluino production in dedicated SUSY searches performed by the ATLAS [62, 63,73-76] and CMS [29,34,77-82] collaborations.

- All slepton mass parameters have been set to the same scale as the first- and second-generation squarks. This choice has no specific experimental or theoretical motivation, and should be regarded as an assumption.

- A decoupling limit with $M_{A}=3 \mathrm{TeV}$ and large $\tan \beta$ values ( $\tan \beta>15$ ) has been assumed. This is partially motivated by results of the LHC searches for higher mass Higgs boson states [83,84].

- For $\tan \beta \gg 1$, the Higgs boson mass depends heavily on the product of the stop-mass parameters $M_{S}=\sqrt{m_{\tilde{t}_{1}} m_{\tilde{t}_{2}}}$ and the mixing between the left- and right-handed states

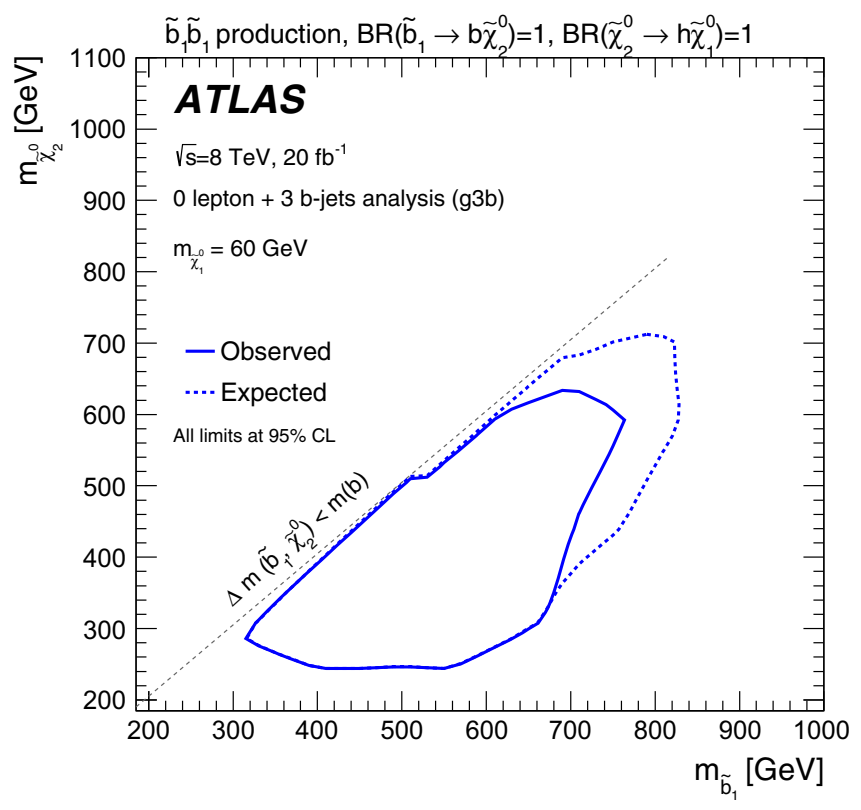

(b)

of $60 \mathrm{GeV}$ (in purple) or $m_{\tilde{\chi}_{1}^{ \pm}}=2 m_{\tilde{\chi}_{1}^{0}}$ (in blue). The limits are shown in the $m_{\tilde{b}_{1}}-m_{\tilde{\chi}_{1}^{ \pm}}$plane. The signal regions used in $\mathbf{b}$ are the $\mathrm{g} 3 \mathrm{~b}-\mathrm{SR}-0 \mathrm{j}$. A fixed neutralino mass of $60 \mathrm{GeV}$ is assumed, and the limit is shown in the $m_{\tilde{b}_{1}}-m_{\tilde{\chi}_{2}^{0}}$ plane

$X_{t}=A_{t}-\mu / \tan \beta$ [85]. The stop sector is therefore completely fixed, given the Higgs boson mass, the value of $X_{t}$ and one of the two stop mass parameters. ${ }^{8}$

- The trilinear couplings $A_{b}$ in the sbottom sector are found to have limited impact on the phenomenology, and are therefore set to zero.

- The gluino mass parameter $M_{3}$ is set such to evade LHC constraints on gluino-pair production.

These assumptions reduce the number of additional free parameters of the model to the mass parameters of the electroweak sector $\left(\mu, M_{1}, M_{2}\right)$ and two of the three third-generation squark mass parameters $\left(m_{\tilde{q} L 3}, m_{\tilde{t} R}, m_{\tilde{b} R}\right)$. All the assumptions made either have a solid experimental basis, or are intended to simplify the interpretation in terms of direct production of stops and sbottoms (as, for example, the assumption on the slepton mass parameters).

Three types of models have been chosen, that, by implementing in different ways constraints arising from naturalness arguments and the dark-matter relic density measurement, further reduce the number of parameters to be scanned over. They are described below, and summarised in Table 3

\footnotetext{
${ }^{8}$ In particular, a minimum value of $M_{S} \sim 800 \mathrm{GeV}$ is allowed if the maximal mixing condition $X_{t} / M_{S}=\sqrt{6}$ is realised.
} 


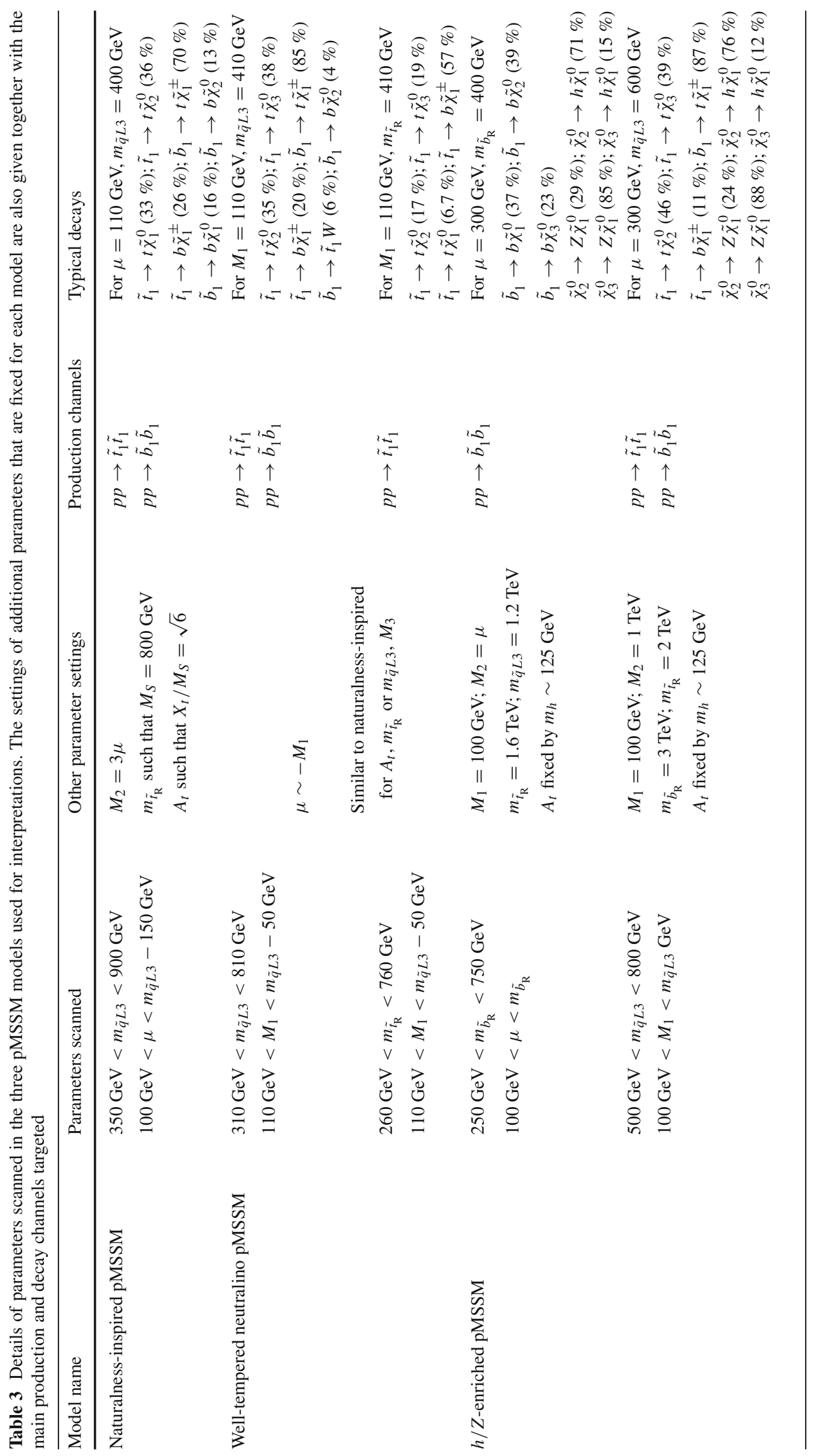


together with additional information on the most relevant production and decay channels.

Naturalness-inspired pMSSM The model is inspired by naturalness criteria, which require a value of $\mu$ in the range of a few hundred $\mathrm{GeV}$, favour stop masses below one $\mathrm{TeV}$, place weak constraints on the gluino mass and give no constraints on the mass of other SUSY particles [86]. The exclusion limits are determined as a function of the higgsino mass parameter $\mu$ and the left-handed squark mass parameter $m_{\tilde{q} L 3}$. The parameter $m_{\tilde{q} L 3}$ is scanned in the range $350 \mathrm{GeV}<$ $m_{\tilde{q} L 3}<900 \mathrm{GeV}$. The parameter $\mu$ is scanned in the range $100 \mathrm{GeV}<\mu<m_{\tilde{q} L 3}-150 \mathrm{GeV}$, where the lower bound is determined by limits on the chargino mass arising from LEP [66-70]. The right-handed stop mass parameter $m_{\tilde{t} R}$ and the stop mixing parameter $X_{t}$ are determined by choosing the maximal mixing scenario $X_{t} / M_{S}=\sqrt{6}$ and by the requirement of having a Higgs boson mass of about $125 \mathrm{GeV}$. The other squark and slepton masses, as well as the bino mass parameter $M_{1}$, are set to $3 \mathrm{TeV}$. The wino mass parameter $M_{2}$ is set such that $M_{2}=3 \mu$. The gluino mass parameter $M_{3}$ is set to $1.7 \mathrm{TeV}$.

With this choice of the model parameters, the spectrum is characterised by two light neutralinos $\left(\tilde{\chi}_{1}^{0}, \tilde{\chi}_{2}^{0}\right)$ and one chargino $\left(\tilde{\chi}_{1}^{ \pm}\right)$, all with masses of the order of $\mu$, a light $\tilde{b}_{1}$ with a mass of the order of $m_{\tilde{q} L 3}$, and a light $\tilde{t}_{1}$ with mass of the order of $m_{\tilde{q} L 3}$ up to $m_{\tilde{q} L 3} \sim 700 \mathrm{GeV}$ (the constraint on $M_{S}$ does not allow the mass of $\tilde{t}_{1}$ to increase beyond about $650 \mathrm{GeV}$ ). The production processes considered are direct pair production of $\tilde{b}_{1}$ and $\tilde{t}_{1}$ with similar masses. Because of the abundance of light higgsino states, many different decays can occur.

Well-tempered neutralino pMSSM The models are designed to loosely satisfy dark-matter thermal-relic density constraints $\left(0.09<\Omega_{\mathrm{c}} h^{2}<0.15\right.$, where $h$ is the Hubble constant), while keeping fine tuning (defined as in Ref. [87]) to less than $1 \%$. The exclusion limits are determined as a function of $M_{1}$ and $m_{\tilde{q} L 3}$, or $M_{1}$ and $m_{\tilde{t} R}$, with $\mu \sim-M_{1}$ in both cases to satisfy the dark-matter constraints through the presence of well-tempered neutralinos [88]. The constraints on the Higgs boson mass are satisfied in a way similar to the naturalness-inspired pMSSM model above. All other parameters are the same as in the naturalness-inspired pMSSM model. These models tend to have three neutralinos and two charginos with masses lower than $\tilde{t}_{1}$ or $\tilde{b}_{1}$, giving rise to a diverse phenomenology.

h/Z-enriched pMSSM These models are defined such that Higgs and $Z$ bosons are produced abundantly in the SUSY particles' decay chains. The assumption of $M_{1}=100 \mathrm{GeV}$ ensures the presence of a bino-like neutralino LSP, while $M_{3}=2.5 \mathrm{TeV}$ ensures that direct gluino production is highly

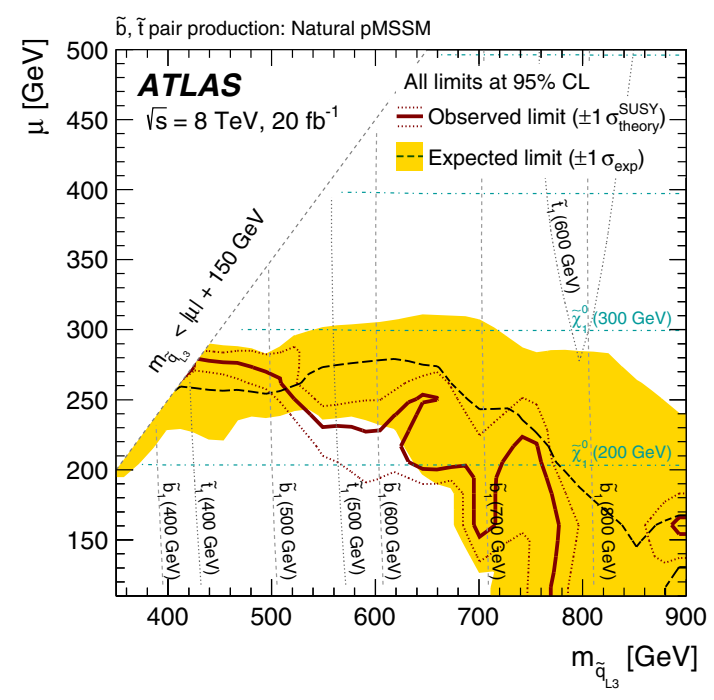

Fig. 13 Expected and observed $95 \% \mathrm{CL}$ exclusion limits for the naturalness-inspired set of pMSSM models from the combination t0L, t1 $\mathrm{L}$ and tb analyses using the signal region yielding the smallest $\mathrm{CL}_{s}$ value for the signal-plus-background hypothesis. The dashed black line indicates the expected limit, and the yellow band indicates the $\pm 1 \sigma$ uncertainties, which include all uncertainties except the theoretical uncertainties in the signal. The red solid line indicates the observed limit, and the red dotted lines indicate the sensitivity to $\pm 1 \sigma$ variations of the signal theoretical uncertainties. The dashed and dotted grey lines indicate a constant value of the stop and sbottom masses, while the dashed light-blue line indicates a constant value of the neutralino mass

suppressed compared to third-generation squark production. Two sets of models have been defined: in the first one, $\mu$ and the right-handed sbottom mass parameter $m_{\tilde{b} R}$ are scanned while keeping $M_{2}=\mu, m_{\tilde{q} L 3}=1.2 \mathrm{TeV}, m_{\tilde{t} R}=1.6 \mathrm{TeV}$; in the second one, $\mu$ and $m_{\tilde{q} L 3}$ are scanned while keeping $M_{2}=1 \mathrm{TeV}, m_{\tilde{b} R}=3 \mathrm{TeV}, m_{\tilde{t} R}=2 \mathrm{TeV}$. The former is dominated by sbottom pair production, while both sbottom and stop pair production are relevant for the latter. Stop mixing parameters are chosen with maximal mixing to satisfy Higgs boson mass constraints. In these models, the decays of the third generation squarks into the heavier neutralino states $\left(\tilde{\chi}_{2}^{0}\right.$ and $\left.\tilde{\chi}_{3}^{0}\right)$ are followed by decays to the lightest neutralino with the emission of a $Z$ or a $h$ boson. Typically the $\tilde{\chi}_{2}^{0}\left(\tilde{\chi}_{3}^{0}\right)$ decays into a $Z$ boson $30 \%(85 \%)$ of the times, and into a Higgs boson $70 \%(15 \%)$ of the times. The subsequent decays of the Higgs boson into $b$-quark pairs (happening with the same branching ratio as in the Standard Model) lead to final states rich in $b$-jets.

Exclusion limits for these pMSSM models are determined by combining many of the SRs defined for the searches discussed in this paper (t0L, t1L, tb, $\left.{ }^{9} \mathrm{t} 2 \mathrm{t} 1 \mathrm{Z}, \mathrm{g} 3 \mathrm{~b}, \mathrm{tc}\right)$. For each

\footnotetext{
9 The tb signal region, discussed in detail in Appendix B.2.3, implement a one-lepton selection, designed to be sensitive to final states containing a top quark, a $b$-quark and $E_{\mathrm{T}}^{\text {miss }}$. It complements the selections of the $t 0 L$ and $t 1 L$ signal regions targeting $t t E_{\mathrm{T}}^{\text {miss }}$ final states.
} 


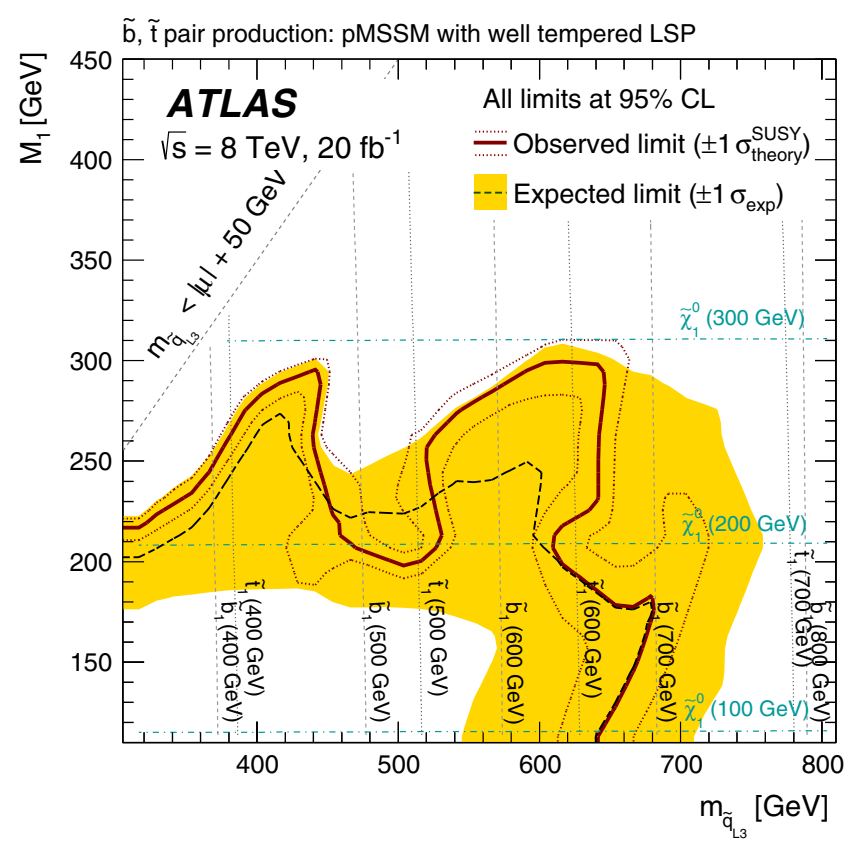

(a)

Fig. 14 Expected and observed $95 \%$ CL exclusion limits for the pMSSM model with well-tempered neutralinos as a function of $M_{1}$ and $\mathbf{a} m_{\tilde{q} L 3}$ or $\mathbf{b} m_{\tilde{t}_{\mathrm{p}}}$. The limit of $\mathbf{a}$ is obtained as the combination of the t0L, t1L, tb and SS3L analyses, while the tOL analysis is used for $\mathbf{b}$. The signal region yielding the smallest $\mathrm{CL}_{s}$ value for the signal-plus-background hypothesis is used for each point. The dashed black line indicates the expected limit, and the yellow band indicates the

set of parameters the individual $95 \%$ CL expected limit is evaluated. The combined exclusion contour is determined by choosing, for each model point, the signal region having the smallest expected $\mathrm{CL}_{S}$ value of the test statistic for the signal-plus-background hypothesis.

Figure 13 shows the exclusion limit for the naturalnessinspired set of pMSSM models based on the $\mathrm{t} 0 \mathrm{~L}, \mathrm{t} 1 \mathrm{~L}$ and tb analyses. The $\mathrm{t} 0 \mathrm{~L}$ and $\mathrm{t} 1 \mathrm{~L}$ analyses have a similar expected sensitivity. These SRs were optimised assuming a $100 \%$ $\mathrm{BR}$ for $\tilde{t}_{1} \rightarrow t \tilde{\chi}_{1}^{0}$ or $\tilde{t}_{1} \rightarrow b \tilde{\chi}_{1}^{ \pm}$, while for these pMSSM models, the stop decays to $\tilde{t}_{1} \rightarrow t \tilde{\chi}_{1}^{0}, \tilde{t}_{1} \rightarrow b \tilde{\chi}_{1}^{ \pm}$and $\tilde{t}_{1} \rightarrow b \tilde{\chi}_{2}^{0}$ with similar branching ratios (and the sbottom to both $\tilde{b}_{1} \rightarrow b \tilde{\chi}_{1}^{0}$ and $\left.\tilde{b}_{1} \rightarrow t \tilde{\chi}_{1}^{ \pm}\right)$. The tb signal regions, discussed in detail in Appendix B.2.3, are designed to be sensitive to final states containing a top quark, a $b$-quark and missing transverse momentum and address such mixeddecay scenarios by requiring a lower jet multiplicity.

The signal regions that dominate the sensitivity are the tb, t0L-SRC1 and t1L-bCd_bulk at low values of $m_{\tilde{q} L 3}$, and tb, t0L-SRA1, t0L-SRA2 and t1L-tNbC_mix at intermediate and high values of $m_{\tilde{q} L 3}$. The excluded region for models with $m_{\tilde{q} L 3} \sim 900 \mathrm{GeV}$ and $\mu \sim 150 \mathrm{GeV}$ is due to the saturation of $m_{\tilde{t}_{1}}$ at high $m_{\tilde{q} L 3}$ values: to satisfy the Higgs

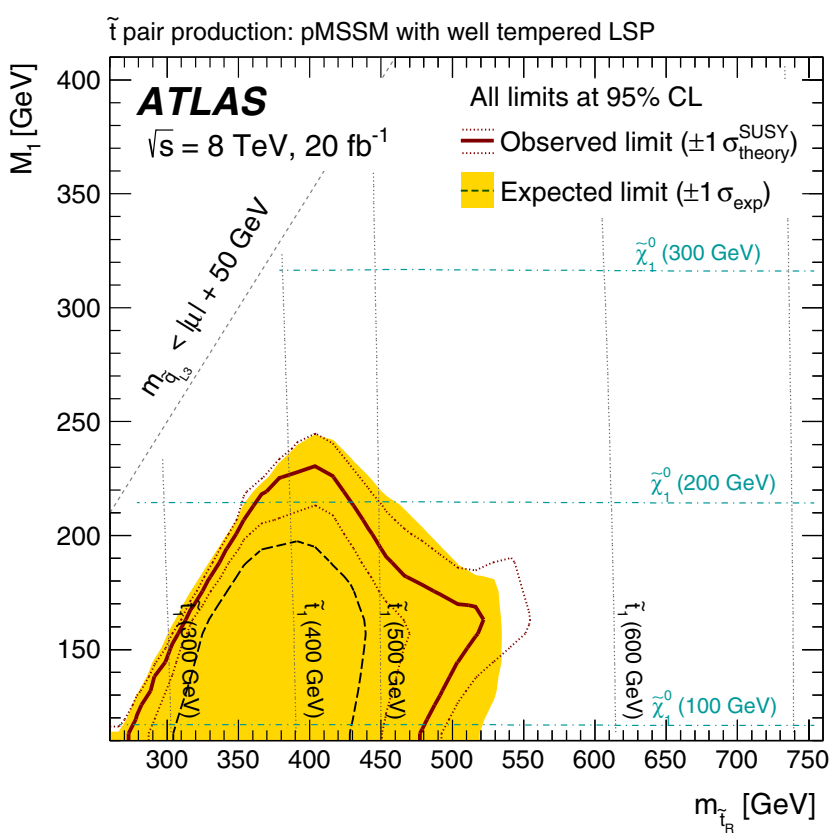

(b)

$\pm 1 \sigma$ uncertainties, which include all uncertainties except the theoretical uncertainties in the signal. The red solid line indicates the observed limit, and the red dotted lines indicate the sensitivity to $\pm 1 \sigma$ variations of the signal theoretical. The dashed and dotted grey lines indicate a constant value of the stop and sbottom masses, while the dashed light-blue line indicates a constant value of the neutralino mass

boson mass constraint requires $M_{S} \sim 800 \mathrm{GeV}$, hence $m_{\tilde{t}_{1}}$ at $m_{\tilde{q} L 3} \sim 900 \mathrm{GeV}$ is smaller than that at $m_{\tilde{q} L 3} \sim 800 \mathrm{GeV}$. The large fluctuations of the observed limit with respect to the expected one are due to transitions between different signal regions providing the best expected exclusion in different regions of the plane.

Figure 14a, b show the exclusion limit obtained for the set of pMSSM models with well-tempered neutralinos as a function of $m_{\tilde{q} L 3}$ and $m_{\tilde{t} R}$, respectively. In both cases, the exclusion is largely dominated by the $\mathrm{t} 0 \mathrm{~L}$ analysis. For Fig. 14a, the signal region dominating the sensitivity at low $m_{\tilde{q} L 3}$ is t0L-SRC1, while at higher $m_{\tilde{q} L 3}$ values t0L-SRA1 and t0L-SRA2 dominate the sensitivity. The drop in sensitivity at $m_{\tilde{q} L 3}=410 \mathrm{GeV}, M_{1}=260 \mathrm{GeV}$ is due to the opening of the $\tilde{t}_{1} \rightarrow t \tilde{\chi}_{2}^{0}$ and $\tilde{t}_{1} \rightarrow t \tilde{\chi}_{3}^{0}$ transition, kinematically suppressed for smaller values of the difference $m_{\tilde{q} L 3}-M_{1}$. Such decays introduce more intermediate states in the decay, effectively reducing the transverse momenta of the final state objects. The large fluctuations of the observed limit are again due to transitions between different signal regions. For Fig. 14b, the sensitivity is entirely dominated by the various tOL-SRC. The difference in sensitivity between these two scenarios is due to the presence of both a stop and 


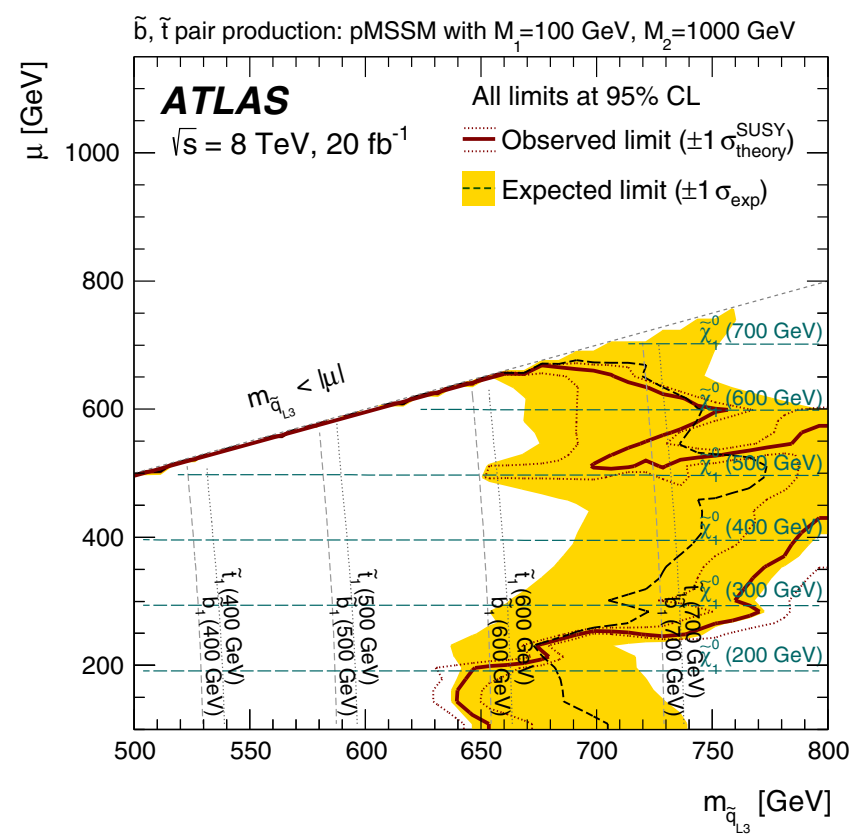

(a)

Fig. 15 Expected and observed $95 \%$ CL exclusion limits for the set of $h / Z$-enriched pMSSM models as a function of $\mu$ and a $m_{\tilde{q} L 3}$ and $\mathbf{b} m_{\tilde{b}_{\mathrm{R}}}$. The limit of $\mathbf{a}$ is obtained as the combination of the t0L, $\mathrm{g} 3 \mathrm{~b}, \mathrm{t} 2 \mathrm{t} 1 \mathrm{Z}$ and SS3L analyses, while the toL, t2t1Z and tb analysis are used for $\mathbf{b}$. The signal region yielding the smallest $\mathrm{CL}_{s}$ value for the signal-plus-background hypothesis is used for each point. The dashed black line indicates the expected limit, and the yellow band

a sbottom for small $m_{\tilde{q} L 3}$, while only a stop is present for low values of $m_{\tilde{t} R}$.

Finally, Fig. 15a, b show the exclusion limit obtained for the set of $h / Z$-enriched pMSSM models. These models yield large $b$-jet multiplicities to the final state through direct sbottom decays, top-quark decays and $\tilde{\chi}_{2}^{0} \rightarrow h / Z \tilde{\chi}_{1}^{0}$. The exclusion is dominated by the t0L and $\mathrm{g} 3 \mathrm{~b}$ analyses for Fig. 15a and by and the tOL analysis for Fig. 15b.

More informations about the limits obtained, including the SLHA files for the points mentioned in Table 3, can be found in Refs. [89] and [90].

\section{Conclusions}

The search programme of the ATLAS collaboration for the direct pair production of stops and sbottoms is summarised and extended by new analyses targeting scenarios not optimally covered by previously published searches. The paper is based on $20 \mathrm{fb}^{-1}$ of proton-proton collisions collected at the LHC by ATLAS in 2012 at a centre-of-mass energy $\sqrt{s}$ $=8 \mathrm{TeV}$. Exclusion limits in the context of simplified models are presented. In general, stop and sbottom masses up to several hundred $\mathrm{GeV}$ are excluded, although the exclusion limits

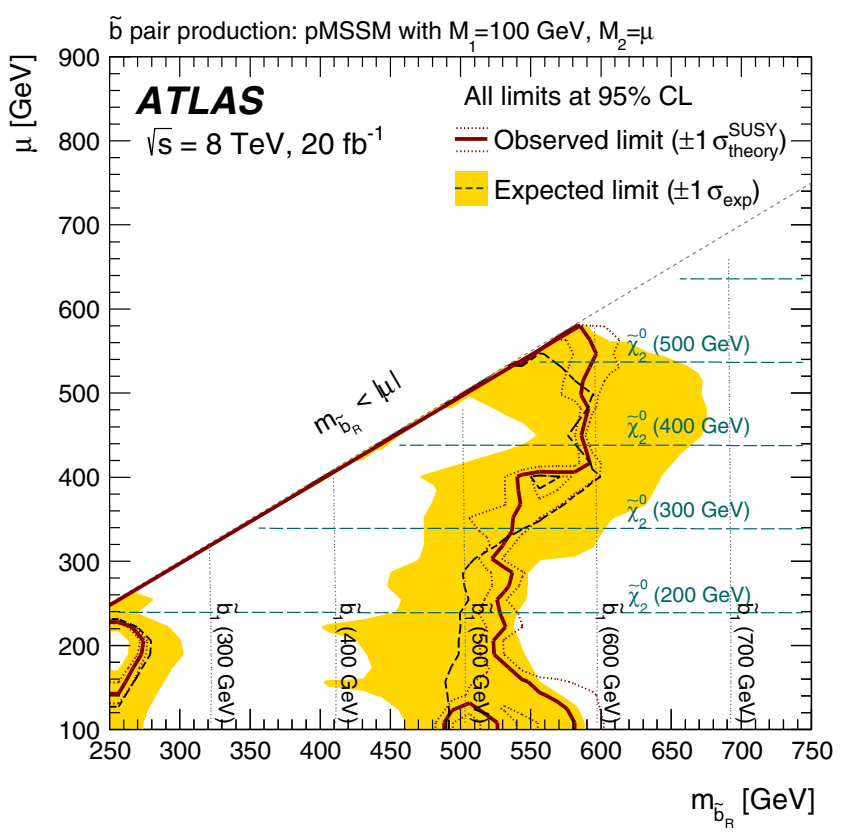

(b)

indicates the $\pm 1 \sigma$ uncertainties, which include all uncertainties except the theoretical uncertainties in the signal. The red solid line indicates the observed limit, and the red dotted lines indicate the sensitivity to $\pm 1 \sigma$ variations of the signal theoretical. The dashed and dotted grey lines indicate a constant value of the stop and sbottom masses, while the dashed light-blue line indicates a constant value of the neutralino mass

significantly weaken in the presence of compressed SUSY mass spectra or multiple allowed decay chains. Three classes of pMSSM models, based on general arguments of Higgs boson mass naturalness and compatibility with the observed dark-matter relic density have also been studied and exclusion limits have been set. Large regions of the considered parameter space are excluded.

We thank CERN for the very successful operation of the LHC, as well as the support staff from our institutions without whom ATLAS could not be operated efficiently.

Acknowledgments We acknowledge the support of ANPCyT, Argentina; YerPhI, Armenia; ARC, Australia; BMWFW and FWF, Austria; ANAS, Azerbaijan; SSTC, Belarus; CNPq and FAPESP, Brazil; NSERC, NRC and CFI, Canada; CERN; CONICYT, Chile; CAS, MOST and NSFC, China; COLCIENCIAS, Colombia; MSMT CR, MPO CR and VSC CR, Czech Republic; DNRF, DNSRC and Lundbeck Foundation, Denmark; EPLANET, ERC and NSRF, European Union; IN2P3-CNRS, CEA-DSM/IRFU, France; GNSF, Georgia; BMBF, DFG, HGF, MPG and AvH Foundation, Germany; GSRT and NSRF, Greece; RGC, Hong Kong SAR, China; ISF, MINERVA, GIF, I-CORE and Benoziyo Center, Israel; INFN, Italy; MEXT and JSPS, Japan; CNRST, Morocco; FOM and NWO, Netherlands; BRF and RCN, Norway; MNiSW and NCN, Poland; GRICES and FCT, Portugal; MNE/IFA, Romania; MES of Russia and NRC KI, Russian Federation; JINR; MSTD, Serbia; MSSR, Slovakia; ARRS and MIZŠ, Slovenia; DST/NRF, South Africa; MINECO, Spain; SRC and Wallenberg Foundation, Sweden; SER, SNSF and Cantons of Bern and 
Geneva, Switzerland; NSC, Taiwan; TAEK, Turkey; STFC, the Royal Society and Leverhulme Trust, UK; DOE and NSF, USA.The crucial computing support from all WLCG partners is acknowledged gratefully, in particular from CERN and the ATLAS Tier-1 facilities at TRIUMF (Canada), NDGF (Denmark, Norway, Sweden), CC-IN2P3 (France), KIT/GridKA (Germany), INFN-CNAF (Italy), NL-T1 (Netherlands), PIC (Spain), ASGC (Taiwan), RAL (UK) and BNL (USA) and in the Tier-2 facilities worldwide.

Open Access This article is distributed under the terms of the Creative Commons Attribution 4.0 International License (http://creativecomm ons.org/licenses/by/4.0/), which permits unrestricted use, distribution, and reproduction in any medium, provided you give appropriate credit to the original author(s) and the source, provide a link to the Creative Commons license, and indicate if changes were made.

Funded by $\mathrm{SCOAP}^{3}$.

\section{A The ATLAS detector and object reconstruction}

The ATLAS detector [91] consists of inner tracking devices surrounded by a superconducting solenoid, electromagnetic and hadronic calorimeters and a muon spectrometer immersed in a toroidal magnetic field. The inner detector (ID), in combination with a superconducting solenoid magnet with a central field of $2 \mathrm{~T}$, provides precision tracking and momentum measurements of charged particles in a pseudorapidity ${ }^{10}$ range $|\eta|<2.5$. The ID consists of a silicon pixel detector, a silicon microstrip detector and a straw tube tracker $(|\eta|<2.0)$ that also provides transition radiation measurements for electron identification. A high-granularity electromagnetic calorimeter system, with acceptance covering $|\eta|<3.2$, uses liquid argon (LAr) as the active medium. A scintillator-tile calorimeter provides hadronic coverage for $|\eta|<1.7$. The end-cap and forward regions, spanning $1.5<|\eta|<4.9$, are instrumented with LAr electromagnetic and hadronic calorimeters. The muon spectrometer has separate trigger and high-precision tracking chambers which provide trigger coverage for $|\eta|<2.4$ and muon identification and momentum measurements for $|\eta|<2.7$.

The data sample used in this analysis was taken during the period from March to December 2012 with the LHC operating at a $p p$ centre-of-mass energy of $\sqrt{s}=8 \mathrm{TeV} .{ }^{11}$ Following requirements based on beam, detector conditions and data quality, the complete dataset corresponds to an inte-

\footnotetext{
10 ATLAS uses a right-handed system with its origin at the nominal interaction point (IP) in the centre of the detector and the $z$-axis along the beam pipe. The $x$-axis points from the IP to the centre of the LHC ring, and the $y$-axis points upward. Cylindrical coordinates $(r, \phi)$ are used in the transverse plane, $\phi$ being the azimuthal angle around the beam pipe. The pseudorapidity is defined in terms of the polar angle $\theta$ as $\eta=-\ln \tan (\theta / 2)$. The distance $\Delta R$ in the $\eta-\phi$ space is defined as $\Delta R=\sqrt{(\Delta \eta)^{2}+(\Delta \phi)^{2}}$.

11 The limits derived using a measurement of the $t \bar{t}$ production cross section discussed in Sect. 4.1 and Appendix B.1 also uses $4.7 \mathrm{fb}^{-1}$ of $p p$ collisions data collected at a centre-of-mass energy of $\sqrt{s}=7 \mathrm{TeV}$.
}

grated luminosity of $20.3 \mathrm{fb}^{-1}$, with an associated uncertainty of $2.8 \%$. The uncertainty is derived following the same methodology as that detailed in Ref. [92]. Events used in the analyses presented in this paper were selected using the ATLAS three-level trigger following different chains based on the signatures being considered. A common set of cleaning cuts, aimed at rejecting events heavily contaminated by non-collision backgrounds, or events containing badly measured or fake jets is applied to all analyses.

The experimental signature of third-generation supersymmetric particles includes the production of $b$-jets in association with missing transverse momentum and possibly additional jets and charged leptons. Different signatures are investigated in this paper to gain sensitivity to a variety of possible topologies arising from the production and decay of stops and sbottoms. Different event selections share common definitions of the final reconstructed objects, which are detailed in the remainder of this Appendix. Analysis-specific departures from those definitions are detailed for each case in Appendix B or in the specific analysis paper.

The reconstructed primary vertex [93] is required to be consistent with the luminous region and to have at least five associated tracks with $p_{\mathrm{T}}>400 \mathrm{MeV}$; when more than one such vertex is found, the vertex with the largest summed $p_{T}^{2}$ of the associated tracks is chosen.

Jets are constructed from three-dimensional clusters of noise-suppressed calorimeter cells [94] using the anti- $k_{t}$ algorithm [95-97] with a distance parameter $R=0.4$ and calibrated with a local cluster weighting algorithm [98]. An area-dependent correction is applied for energy from additional proton-proton collisions based on an estimate of the pileup activity in a given event using the method proposed in Ref. [99]. Jets are calibrated as discussed in Ref. [100] and required to have $p_{\mathrm{T}}>20 \mathrm{GeV}$ and $|\eta|<4.5$. Events containing jets arising from detector noise, cosmic-ray muons, or other non-collision sources are removed from consideration [100].

Jets arising from a $b$-quark fragmentation and within the acceptance of the inner detector $(|\eta|<2.5)$ are identified with an algorithm that exploits both the track impact parameters and secondary vertex information [101]; this algorithm is based on a neural network using the output weights of the IP3D, JetFitter+IP3D, and SV1 algorithms (defined in Refs. [102,103]). A lower cut on the output of the neural network defines the $b$-tagged jets. Three different working points are used, with a nominal efficiency of 60,70 and $80 \%$ as evaluated on simulated top quark pair production events. The corresponding rejection factors against jets originating from light $(c)$ quarks are 25 (3), 135 (5) and 600 (8).

Electrons are reconstructed from energy clusters in the electromagnetic calorimeter matched to a track in the inner detector [104] and are required to have $|\eta|<2.47$. Several criteria, including calorimeter shower shape, quality 
of the match between the track and the cluster, and the amount of transition radiation emitted in the TRT detector, are used to define three selections with decreasing efficiency and increasing purity, named respectively 'loose', 'medium' and 'tight' [104]. These three electron selections are used throughout this paper in the definitions of various signal and control regions. Muons, which are identified either as a combined track in the muon spectrometer and inner detector systems, or as an inner detector track matched with a muon spectrometer track segment $[105,106]$, are required to have $|\eta|<2.4$.

Electrons and muons (generically referred to by the symbol $\ell$ ) are usually required to have transverse momentum $p_{\mathrm{T}}>10 \mathrm{GeV}$. For specific scenarios with compressed mass spectra, low- $p_{\mathrm{T}}$ leptons are expected and the $p_{\mathrm{T}}$ threshold is lowered to $6 \mathrm{GeV}$ for muons and to $7 \mathrm{GeV}$ for electrons.

The missing transverse momentum $\mathbf{p}_{\mathrm{T}}^{\text {miss }}$ (with magnitude $E_{\mathrm{T}}^{\text {miss }}$ ) is the negative vector sum of the $p_{\mathrm{T}}$ measured in the clusters of calorimeter cells, which are calibrated according to their associated reconstructed object (e.g. jets and electrons), and the $p_{\mathrm{T}}$ of the muons. Calorimeter cells not associated with any reconstructed object are also used in the calculation of $\mathbf{p}_{\mathrm{T}}^{\text {miss }}$. The missing transverse momentum from the tracking system (denoted by $\mathbf{p}_{\mathrm{T}}^{\text {miss, track }}$, with magnitude $E_{\mathrm{T}}^{\text {miss,track }}$ ) is computed from the vector sum of the reconstructed inner detector tracks with $p_{\mathrm{T}}>500 \mathrm{MeV}$ and $|\eta|<2.5$, associated with the primary vertex in the event.

\section{B Analyses used in the paper}

Several signal regions are used in this paper, either standalone or in combination with others, to derive exclusion limits in the many models considered. This Appendix provides a review of the already published analyses and a more extended documentation of the signal regions not previously published.

\section{B.1 Review of already published signal regions}

The discussion of analyses that have already been published is reduced to a summary for the sake of brevity. Table 1 provides a reference to the papers where full details of the signal, control and validation region selections, together with the strategies adopted for the estimation of the background processes are found.

Multijet final states (t0L) The analysis is designed to be sensitive to final states arising from all-hadronic decays of directly pair-produced stops [16]. Two sets of signal regions were optimised to maximise the sensitivity to topologies arising from $\tilde{t}_{1} \rightarrow t \tilde{\chi}_{1}^{0}$ decays, assumed to happen with a branching ratio of one. The first set of signal regions, named t $0 \mathrm{~L}-$
SRA, assumes that both top quark hadronic decays can be fully resolved by indentifying the six final-state jets. The SM background [dominated by $t \bar{t}$ and $Z+$ heavy flavour (HF) jets production] is rejected based on the presence of two hadronic systems consistent with top quarks and large $E_{\mathrm{T}}^{\text {miss }}$. The second set of signal regions, named t0L-SRB targets a similar scenario, but aims at topologies where the top quarks have a large boost, and some of the decay products are merged into a single jet. The event selection is designed to select final states with a maximum of five $R=0.4$ anti- $k_{t}$ jets, to be mutually exclusive with t0L-SRA, and relies on the presence of $R=0.8$ and $R=1.2$ anti- $k_{t}$ jets containing the hadronic decay products of the two top quarks. The jet masses, the transverse mass of the $E_{\mathrm{T}}^{\text {miss }}$ and the nearest $b$-jet, and other variables are used to discriminate against the dominant SM $t \bar{t}, Z+\mathrm{HF}$ jets and $W+\mathrm{HF}$ jets production background processes.

Finally, a third set of signal regions, named tOL-SRC, is designed to increase the analysis sensitivity to the decay $\tilde{t}_{1} \rightarrow$ $b \tilde{\chi}_{1}^{ \pm}$. The presence of the intermediate chargino state tends to decrease the jet multiplicity: these signal regions require five anti- $k_{t}$ jets with $R=0.4$, and base the signal selection on a set of transverse mass variables aimed at rejecting the dominant SM $t \bar{t}$ production process.

One-lepton final states (t1L) The large number of signal regions defined in this analysis stems from the variety and complexity of the possible stop final states considered [17]. All signal regions are characterised by the presence of one lepton, a second-lepton veto, a minimum of two jets and large $E_{\mathrm{T}}^{\mathrm{miss}}$. A first set of four signal regions (t1L-tN) were optimised assuming a branching ratio of $100 \%$ for the decay $\tilde{t} \rightarrow t \tilde{\chi}_{1}^{0}$. These signal regions aim at having sensitivity to different $\Delta m\left(\tilde{t}, \tilde{\chi}_{1}^{0}\right)$, in particular t1L-tN_diag targets scenarios with small $\Delta m\left(\tilde{t}_{1}, \tilde{\chi}_{1}^{0}\right)$ and makes use of the shape information of the $E_{\mathrm{T}}^{\text {miss }}$ and $m_{\mathrm{T}}$ distributions. ${ }^{12}$ The $\mathrm{t} 1 \mathrm{~L}-$ $\mathrm{tN} \_$boost SR targets models with the largest $\Delta m\left(\tilde{t}, \tilde{\chi}_{1}^{0}\right)$, where the top quark produced by the stop decay has a large boost and large- $R$ jets are used to reconstruct the top quark decays.

The decay $\tilde{t} \rightarrow \tilde{\chi}_{1}^{ \pm} b$ introduces additional degrees of freedom in the decay. The final-state kinematics is largely driven by the mass separation between the stop and the chargino $\Delta m\left(\tilde{t}, \tilde{\chi}_{1}^{ \pm}\right)$, and by that between the chargino and the neutralino $\Delta m\left(\tilde{\chi}_{1}^{ \pm}, \tilde{\chi}_{1}^{0}\right)$. Several signal regions, identified by the

\footnotetext{
12 The transverse mass $m_{\mathrm{T}}$ of the lepton with transverse momentum $\mathbf{p}_{\mathrm{T}}$ and the missing transverse momentum vector $\mathbf{p}_{\mathrm{T}}^{\text {miss }}$ with magnitude $E_{\mathrm{T}}^{\text {miss }}$ is defined as

$m_{\mathrm{T}}=\sqrt{2\left(\left|\mathbf{p}_{\mathrm{T}}\right| E_{\mathrm{T}}^{\mathrm{miss}}-\mathbf{p}_{\mathrm{T}} \cdot \mathbf{p}_{\mathrm{T}}^{\mathrm{miss}}\right)}$

and it is extensively used in one-lepton final states to reject SM background processes containing a $W$ boson decaying leptonically.
} 
prefix $\mathrm{t} 1 \mathrm{~L}-\mathrm{bC}$ were designed and optimised depending on the mass hierarchy and, consequently, on the different kinematics of the lepton and $b$-jets.

The four signal regions t1L-bCa_low, t1L-bCa_med, t1L-bCb_med1 and t1L-bCb_high target small values of $\Delta m\left(\tilde{\chi}_{1}^{ \pm}, \tilde{\chi}_{1}^{0}\right)$ and have the common feature of making use of a dedicated soft-lepton selection: muons and electrons are identified down to a $p_{\mathrm{T}}$ threshold of 6 and $7 \mathrm{GeV}$, respectively, requiring a special treatment for the estimate of possible background processes arising from lepton misidentification. They are collectively referred to as "soft-lepton" signal regions. Both t1L-bCa signal regions require a hard ISR jet to boost the stop pair system and produce a sizeable $E_{\mathrm{T}}^{\text {miss }}$. The $1 \mathrm{~L}-\mathrm{bCb}$ targets large values of $\Delta m\left(\tilde{t}_{1}, \tilde{\chi}_{1}^{ \pm}\right)$and exploits the presence of two relatively hard $b$-jets in the event.

The signal region t1L-bCc_diag targets a mass hierarchy complementary to that of the t1L-bCb. The small value of $\Delta m\left(\tilde{t}, \tilde{\chi}_{1}^{ \pm}\right)$gives rise to soft $b$-jets that go undetected, hence $b$-tagged jets are vetoed for this region.

Topologies arising from scenarios where both $\Delta m\left(\tilde{t}, \tilde{\chi}_{1}^{ \pm}\right)$ and $\Delta m\left(\tilde{\chi}_{1}^{ \pm}, \tilde{\chi}_{1}^{0}\right)$ are sizeable are targeted by the three t1LbCd regions: they all require four jets in the final state, are characterised by different $b$-jet multiplicities, and apply different selections on the $E_{\mathrm{T}}^{\text {miss }}, m_{\mathrm{T}}$ and $a m_{\mathrm{T} 2}{ }^{13}$ variables. A veto on additional isolated tracks and $\tau$ lepton candidates identified with loose criteria helps to suppress the dominant SM background from dileptonic $t \bar{t}$ decays.

The last two signal regions listed in Table 1, t1L-3body and t1L-tNbC_mix, were optimised for two additional possible scenarios. If $\Delta m\left(\tilde{t}, \tilde{\chi}_{1}^{0}\right)<m_{t}$ and the mass hierarchy or the model parameters suppress the decay through a chargino, then the dominant stop decay is $\tilde{t} \rightarrow b W \tilde{\chi}_{1}^{0}$, through an off-shell top quark (three-body decay). The dedicated signal region relies on the shape information from the $m_{\mathrm{T}}$ and $a m_{\mathrm{T} 2}$ variable distributions. Finally, t1L-tNbC_mix is designed to recover sensitivity in scenarios where the stop is assumed to decay with similar probabilities to $t \tilde{\chi}_{1}^{0}$ and $b \tilde{\chi}_{1}^{ \pm}$: the selection aims to reject the dominant dileptonic $t \bar{t}$ background by making use of the topness [109] variable.

Two-lepton final states (t2L) If the SUSY mass hierarchy forbids the presence of sleptons in the stop decay chain, final states containing two leptons ( $e$ or $\mu$ ) and a large amount of $E_{\mathrm{T}}^{\mathrm{miss}}$ would arise from stop pair production. The main background is given by SM processes containing two $W$ bosons in the final state (mainly $t \bar{t}$ and $W W$ ) [18]. To discrimi-

\footnotetext{
13 The asymmetric stransverse mass variable is a variant of the stransverse mass variable $[107,108]$ defined to efficiently reject dileptonic $t \bar{t}$ decays. It assumes that the undetected particle is the $W$ boson for the branch with the lost lepton and the neutrino is the missing particle for the branch with the observed charged lepton. For the dileptonic $t \overline{\mathrm{t}}$ events, $a m_{\mathrm{T} 2}$ is bounded from above by the top quark mass, whereas new physics can exceed this bound.
}

nate the stop signal from the SM background, the stransverse mass variable $m_{\mathrm{T} 2}[107,108]$ is used. The stransverse mass, computed using the two leptons as visible particles and the missing transverse momentum vector, exhibits a kinematical end-point at $m_{W}$ for most SM processes. Because of the presence of additional $E_{\mathrm{T}}^{\text {miss }}$ due to the LSP, the end-point for a SUSY signal can be at larger values, depending on the mass separation between the particles involved in the decay. The analysis is optimised assuming $\tilde{t}_{1} \rightarrow \tilde{\chi}_{1}^{ \pm} b$ with $\mathrm{BR}=100 \%$ and $\Delta m\left(\tilde{\chi}_{1}^{ \pm}, \tilde{\chi}_{1}^{0}\right)>m_{W}$, but it is also sensitive to the threebody decay mode of the stop. To derive exclusion limits, five signal regions (t2L) have been defined, requiring different jet multiplicities and different $m_{\mathrm{T} 2}$ thresholds. A selection requiring two $b$-jets and based on $m_{\mathrm{T} 2}$ computed using them as visible particles is sensitive to the chargino decay mode with $\Delta m\left(\tilde{t}_{1}, \tilde{\chi}_{1}^{ \pm}\right)>m_{t}$. Finally, a multivariate discriminant is built which targets the $\tilde{t}_{1} \rightarrow t \tilde{\chi}_{1}^{0}$ decay mode.

Final states from compressed stop decays (tc) If the difference in mass between the stop and the neutralino is smaller than the $W$ boson mass, then the only possible decay channels are $\tilde{t} \rightarrow \tilde{\chi}_{1}^{0} c$ or $\tilde{t} \rightarrow W^{*} b$, where the decay products of the off-shell $W^{*}$ would, in general, be soft. This analysis [19] has defined two sets of signal regions, both optimised for the $\tilde{t} \rightarrow \tilde{\chi}_{1}^{0} c$ decay. A common preselection requires the presence of a high- $p_{\mathrm{T}}$ jet, large $E_{\mathrm{T}}^{\mathrm{miss}}$ and applies a lepton veto. The first set of signal regions named tc-M, targets scenarios with the stop mass almost degenerate with the neutralino mass, and applies a selection that exploits a monojet-like signature arising from the presence of an ISR jet. Three different signal regions have been designed, characterised by increasing thresholds on the leading jet $p_{\mathrm{T}}$ and $E_{\mathrm{T}}^{\mathrm{miss}}$. The second set of signal regions, named tc-C, targets less compressed scenarios, and exploits the presence of jets originating from the fragmentation of $c$-quarks in the final state. A dedicated $c$-tagging algorithm was used to reject the dominant SM background processes arising mostly from $t \bar{t}$ and $Z \rightarrow v \bar{v}$ (produced in association with heavy-flavour jets) production. As in the case of the tc-M signal regions, different thresholds on the leading jet $p_{\mathrm{T}}$ and on $E_{\mathrm{T}}^{\text {miss }}$ are used to identify a looser and a tighter tc-C region.

Final states with a $Z$ boson (t2t1Z) A $Z$ boson can be emitted in the decay of $\tilde{t}_{2} \rightarrow \tilde{t}_{1} Z$, producing final states with large lepton multiplicities. It can be useful to look for $\tilde{t}_{2}$ (rather than $\tilde{t}_{1}$ ) production if, for example, the mass of $\tilde{t}_{1}$ is very close to the sum of the top quark and neutralino masses, which would lead to $\tilde{t}_{1}$ pair production final states difficult to distinguish from SM $t \bar{t}$ production. Models are investigated with $\Delta m\left(\tilde{t}_{1}, \tilde{\chi}_{1}^{0}\right)=180 \mathrm{GeV}$ with the decay $\tilde{t}_{1} \rightarrow t \tilde{\chi}_{1}^{0}$. The final state would contain, beyond the $\mathrm{Z}$ boson, several jets arising from the $\tilde{t}_{1}$ decay. Similar final states can be obtained in GMSB models where the $Z$ boson is emitted 
in the $\tilde{\chi}_{1}^{0} \rightarrow \tilde{G} Z$ decay if the gravitino $\tilde{G}$ is the LSP and the neutralino the NLSP.

This analysis [20] defines five different signal regions divided into two sets. The first set, named t2t1Z-SR2, requires two same-flavour leptons whose invariant mass is consistent with that of a $Z$ boson, $m_{Z}$, and at least one $b$-tagged jet. The three signal regions are characterised by the different selection thresholds applied to the $E_{\mathrm{T}}^{\text {miss }}$, to the transverse momentum of the dilepton system $p_{\mathrm{T}}(\ell \ell)$ and to the jet multiplicity. The second set of signal regions, named t2t1Z-SR3, requires three leptons, two of which must form an oppositesign same-flavour pair whose invariant mass is consistent with $m_{Z}$. Both signal regions require at least five jets, among which at least one has to be $b$-tagged. The two signal regions are characterised by the different selection thresholds applied to $p_{\mathrm{T}}(\ell \ell)$ and to the leading lepton $p_{\mathrm{T}}$.

Final states with two $b$-jets and $E_{\mathrm{T}}^{\text {miss }}(\mathrm{b} 0 \mathrm{~L})$ This signature arises naturally from the sbottom decay $\tilde{b}_{1} \rightarrow b \tilde{\chi}_{1}^{0}$. Moreover, one expects the same final state from $\tilde{t}_{1} \rightarrow \tilde{\chi}_{1}^{ \pm} b$ followed by $\tilde{\chi}_{1}^{ \pm} \rightarrow f f^{\prime} \tilde{\chi}_{1}^{0}$ in the limit of small $\Delta m\left(\tilde{\chi}_{1}^{ \pm}, \tilde{\chi}_{1}^{0}\right)$. This analysis [21] defines two sets of signal regions, b0LSRA and b0L-SRB, targeting scenarios with large and small squark-neutralino mass separations, respectively.

The event selection of b0L-SRA requires large $E_{\mathrm{T}}^{\text {miss }}$, exactly two $b$-jets and vetoes the presence of additional jets; the rejection of the SM $t \bar{t}$ production background is carried out by making use of the contransverse mass [110] of the two $b$-jets. Its distributions shows a kinematical end-point at about $135 \mathrm{GeV}$ for $t \bar{t}$ production, while extending to higher values for the signal.

A selection relying on the presence of an ISR jet is instead needed if the third-generation squark mass is almost degenerate with that of the neutralino. This is the purpose of b0L$\mathrm{SRB}$, which selects a hard, non- $b$-tagged leading jet recoiling against the squark pair system. The selection includes the requirement of two $b$-tagged jets, a veto on additional hadronic activity, and the presence of large $E_{\mathrm{T}}^{\mathrm{miss}}$.

Final states with three $\boldsymbol{b}$-jets (g3b) This analysis [62] is designed to search for gluino-mediated sbottom and stop production in events with no leptons or one lepton (electron or muon) in the final state. However, it was found to have sensitivity for direct $\tilde{b}_{1}$ production followed by $\tilde{b}_{1} \rightarrow \tilde{\chi}_{2}^{0} b \rightarrow \tilde{\chi}_{1}^{0} h b$, where $h$ is the SM Higgs boson with mass $m_{h}=125 \mathrm{GeV}$, and also sensitivity to some of the pMSSM models considered in this paper. Such final states are characterised by a large multiplicity of $b$-jets both in $\tilde{g} \tilde{g} \rightarrow \tilde{t}_{1} \tilde{t}_{1} t t$ and $\tilde{g} \tilde{g} \rightarrow \tilde{b}_{1} \tilde{b}_{1} b b$ where there are up to four $b$-jets in the final state.

Three sets of signal regions have been designed to target different mass hierarchies of the gluino-mediated sbottom and stop production models. All signal regions have at least four jets with $p_{\mathrm{T}}>30 \mathrm{GeV}$, three identified $b$-jets, large $E_{\mathrm{T}}^{\text {miss }}$ and a large $m_{\mathrm{eff}}$, defined as the scalar sum of the $p_{\mathrm{T}}$ of the jets and $E_{\mathrm{T}}^{\mathrm{miss}}$.

Strongly produced final states with two same sign or three leptons (SS3L) Final states containing many leptons or same-sign (SS) leptons can arise from the pair production of gluinos and squarks, when the produced particles decay to the LSP through multiple intermediate stages, or when several top quarks appear as part of the decay chain. The analysis was developed for the gluino-mediated stop production process $\tilde{g} \tilde{g} \rightarrow \tilde{t}_{1} \tilde{t}_{1} t t$ followed by $\tilde{t}_{1} \rightarrow t \tilde{\chi}_{1}^{0}$, which can yield final states containing up to four leptons, including SS pairs. Similar final states arise from the sbottom decay $\tilde{b}_{1} \rightarrow t \tilde{\chi}_{1}^{ \pm}$, which are studied in this paper.

This analysis [63] concentrates on final states containing either three leptons or a SS lepton pair produced in association with many jets. Five signal regions (identified by the prefix SS3L) are defined, which are characterised by different light- and heavy-flavour jet multiplicities, high selection thresholds on $E_{\mathrm{T}}^{\text {miss }}$ and $m_{\mathrm{eff}}$, and different thresholds on the transverse mass of the lepton with the highest transverse momentum and the $E_{\mathrm{T}}^{\text {miss }}$.

Spin correlation in $t \bar{t}$ production events (SC) If the mass of the $\tilde{t}_{1}$ is such that $m_{\tilde{t}_{1}} \sim m_{\tilde{\chi}_{1}^{0}}+m_{t}$, the final-state kinematics are similar to that of Standard Model $t \bar{t}$ production. One possible approach is to derive exclusion limits on the stop mass by performing SM precision measurements. This analysis has measured the azimuthal angle difference between the two leptons arising from the dileptonic $t \bar{t}$ decay [64]. The events are required to contain, beside the two leptons, at least two additional jets, one of which is required to be $b$ tagged. In events containing two leptons of the same flavour, the $Z$ production background is suppressed by applying a selection on the dilepton invariant mass. The distribution of the azimuthal angle between the two leptons is sensitive to the spin correlations of the $t \bar{t}$ system: it is hence used to extract limits on possible contaminations from direct scalar top production events.

$t \bar{t}$ production cross section (xsec) The measurement of the $t \bar{t}$ production cross section using events containing two different-flavour leptons $e \mu$ and $b$-tagged jets is used in Ref. [65] to extract limits on the direct pair production of $\tilde{t}_{1}$ with mass close to the top quark. The assumed decay is $\tilde{t}_{1} \rightarrow t \tilde{\chi}_{1}^{0}$.

The $t \bar{t}$ production cross section $\sigma_{t \bar{t}}$ is obtained by using the equations

$$
\begin{aligned}
& N_{1}=L \sigma_{t \bar{t}} \epsilon_{e \mu} 2 \epsilon_{b}\left(1-C_{b} \epsilon_{b}\right)+N_{1}^{\mathrm{bkg}} \\
& N_{2}=L \sigma_{t \bar{t}} \epsilon_{e \mu} C_{b} \epsilon_{b}^{2}+N_{2}^{\mathrm{bkg}}
\end{aligned}
$$


where $N_{1}$ and $N_{2}$ are the number of events with two different flavour leptons having exactly one or two $b$-tagged jets, respectively, $L$ is the integrated luminosity, $\epsilon_{e \mu}$ the efficiency for a $t \bar{t}$ event to pass the lepton selection, $\epsilon_{b}$ is the probability of having a $b$-jet within acceptance and for it to be tagged, $C_{b}$ is a correlation coefficient which is close to unity, and $N_{1}^{\mathrm{bkg}}$ and $N_{2}^{\text {bkg }}$ are the number of events with one or two $b$-tagged jets from SM events different from $t \bar{t}$ production. The values of $\sigma_{t \bar{t}}$ and $\epsilon_{b}$ are extracted from the data by solving the two simultaneous Eqs. (2) and (3), avoiding the need to estimate $\epsilon_{b}$ from simulation.

Stop-pair production events with $m_{\tilde{t}_{1}}>m_{t}+m_{\tilde{\chi}_{1}^{0}}$ have similar $\epsilon_{e \mu}$ and $b$-jet kinematics to SM $t \bar{t}$ production events, so the fitted value of $\epsilon_{b}$ in a combined sample is compatible with that from $t \bar{t}$ production events alone, and the fitted cross section corresponds closely to the sum of $t \bar{t}$ and stop-pair production cross sections. Limits on stop pair production are extracted by calculating $95 \%$ CL limits on the stop pair production signal strength $\mu$ (defined as the ratio of the obtained stop cross section to the theoretical prediction) based on the comparison of the measured cross section with that predicted for SM $t \bar{t}$ production events alone. A $95 \%$ CL signal strength smaller than unity for a given signal point implies its exclusion.

This interpretation, which made use of collision data with both $\sqrt{s}=7$ and $8 \mathrm{TeV}$, is extended here to the three-body decay $\tilde{t}_{1} \rightarrow W b \tilde{\chi}_{1}^{0}$. The main difference with respect to the scenario considered in Ref. [65] is that the three-body decay tends to yield $b$-jets with lower $p_{\mathrm{T}}$, leading to a fitted $\epsilon_{b}$ for the combined sample which is different from that expected for $t \bar{t}$ events alone. The limits obtained are summarised in Fig. 16 for a neutralino mass of $1 \mathrm{GeV}$. A $95 \%$ CL limit that excludes stop masses below $175 \mathrm{GeV}$ is obtained. The figure also shows the effect on the limit of a "sneaky top squark" scenario [111]: the presence of a $\tilde{t}_{1}$ with mass similar to that of the top quark could bias the measurement of the top-quark mass itself. The bias in the top-mass measurement introduced by the existence of a $\tilde{t}_{1}$ with mass $m_{\tilde{t}_{1}}=170 \mathrm{GeV}$ depends on the analysis technique and channel, and was evaluated to be at most $1 \mathrm{GeV}$ for the two- and three-dimensional template techniques used in the ATLAS top mass measurement in the lepton+jets channel [112]. The effect of a potential bias of 1 and $2.5 \mathrm{GeV}$ on the top-mass measurement was studied by recalculating the observed $95 \%$ CL limit on $\mu$ when reducing the predicted SM $t \bar{t}$ production cross section from the baseline value of $m_{t}=172.5 \pm 1.0 \mathrm{GeV}$ to those obtained for top mass central values of 173.5 and $175 \mathrm{GeV}$. The corresponding limit on the stop mass is reduced by about 5 and $15 \mathrm{GeV}$, respectively.

The dependence of the exclusion limits on the neutralino mass was studied and found to be important: the effect of an increasing neutralino mass is to decrease the $p_{\mathrm{T}}$ of the $b$-jets,

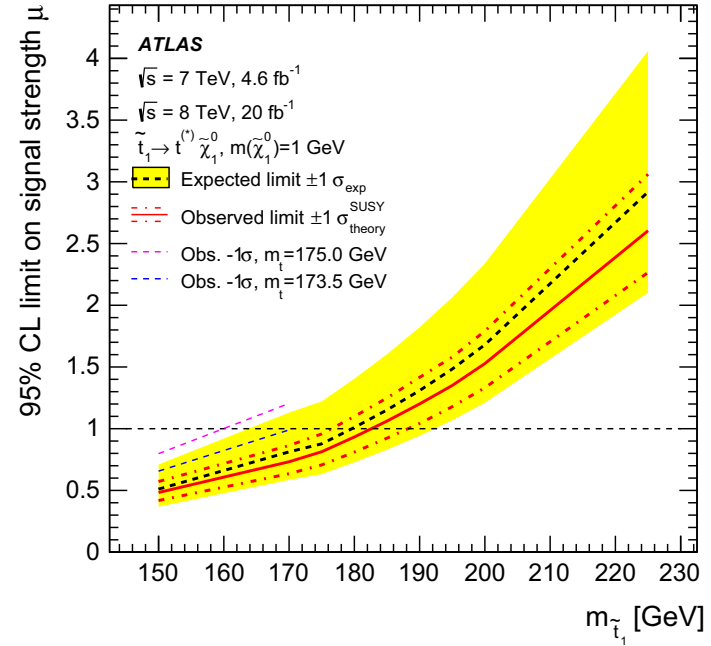

Fig. 16 Expected and observed 95\% CL limits on the signal strength $\mu$ (defined as the ratio of the obtained stop cross section to the theoretical prediction) for the production of $\tilde{t}_{1}$ pairs as a function of $m_{\tilde{t}_{1}}$. The stop is assumed to decay as $\tilde{t}_{1} \rightarrow t \tilde{\chi}_{1}^{0}$ or through its three-body decay depending on its mass. The neutralino is assumed to have a mass of $1 \mathrm{GeV}$. The black dotted line shows the expected limit with $\pm 1 \sigma$ uncertainty band shaded in yellow, taking into account all uncertainties except the theoretical cross-section uncertainties on the signal. The red solid line shows the observed limit, with dotted lines indicating the changes as the nominal signal cross section is scaled up and down by its theoretical uncertainty. The short blue and purple dashed lines indicate how the observed limits with the signal cross section reduced by one standard deviation of its theoretical uncertainty for $m_{\tilde{t}_{1}}<m_{t}$ when the top quark mass is assumed instead to be $173.5 \pm 1.0$ and $175.0 \pm 1.0 \mathrm{GeV}$

and hence to lower the value of $\epsilon_{b}$ for the stop pair production signal. For a neutralino mass of $30 \mathrm{GeV}$, only a small range of stop masses around $150 \mathrm{GeV}$ is excluded.

The sensitivity of the $t \bar{t}$ cross-section measurement to $\tilde{t}_{1}$ pair production assuming a branching ratio of $100 \%$ into $\tilde{t}_{1} \rightarrow b \tilde{\chi}_{1}^{ \pm}$, followed by $\tilde{\chi}_{1}^{ \pm} \rightarrow W^{(*)} \tilde{\chi}_{1}^{0}$ with $m_{\tilde{\chi}_{1}^{ \pm}}=2 m_{\tilde{\chi}_{1}^{0}}$ was also investigated. The presence of the intermediate chargino state tends to lower the $p_{\mathrm{T}}$ of the leptons and of the $b$-jets significantly, hence decreasing both $\epsilon_{e \mu}$ and $\epsilon_{b}$. No exclusion limit can be derived for this scenario.

Summarising, the limits on stop pair production obtained in Ref. [65] have been extended by considering the stop threebody decay. Stop masses between $150 \mathrm{GeV}$ and $m_{t}$ can be excluded for a neutralino mass of $1 \mathrm{GeV}$. The exclusion holds provided that any bias in the top-quark mass measurement by a nearby stop is not significant. Studies indicate that this potential bias would affect the limit on the stop mass by less than $5 \mathrm{GeV}$.

\section{B.2 Description of the new signal regions}

New analyses were developed to target topologies and regions of the SUSY parameter space not well covered 
by previously published signal regions. They are identified throughout this paper and in Table 1 with the acronym WW, tb and $\mathrm{t} 2 \mathrm{t} 1 \mathrm{~h}$. Their contribution to the exclusion limits derived both in simplified and pMSSM models is outlined in Sects. 4 and 5 respectively. In this Appendix, further details about these analyses are provided for the interested reader. Additional informations about selection efficiencies, sensitivities of the different signal regions and individual limit plots, please refer to Refs. $[89,90]$.

\section{B.2.1 Final states with two leptons at intermediate values of $m_{T 2}(W W)$}

The measurement of the production cross section of nonresonant $W W$ pairs in the two-lepton channel at the LHC [113-115] has given rise to theoretical speculations [116118] which interpret the possible excess as due to the production of a light stop. The mass hierarchy favoured by these speculations includes a $\tilde{t}_{1}$ with mass around $200 \mathrm{GeV}$, a $\tilde{\chi}_{1}^{ \pm}$ degenerate with it, and $m_{\tilde{\chi}_{1}^{ \pm}}-m_{\tilde{\chi}_{1}^{0}}$ of a few tens of GeV: possible hadronic decay products of the $\tilde{t}_{1} \rightarrow b \tilde{\chi}_{1}^{ \pm}$transition would have low $p_{\mathrm{T}}$ and would allow the events to survive the tight jet-veto selections applied in the SM cross-section measurement. Dedicated signal regions, defined by requiring two different-flavour opposite-sign leptons in the final states, are designed to have maximum sensitivity to such scenarios. The approach is also sensitive to scenarios where the stop decays predominantly through the three-body $\tilde{t}_{1} \rightarrow b W \tilde{\chi}_{1}^{0}$ or four-body $\tilde{t}_{1} \rightarrow b \ell \nu \tilde{\chi}_{1}^{0}$ decay.

MC simulated events are used to model the signal and to describe all backgrounds that produce two prompt leptons from $W, Z$ or $h$ decay. For processes whose predicted yield in the signal regions is small, or whose topology resembles very closely that of the signal, making it hard to define a proper control region, the background estimate is fully based on $\mathrm{MC}$ simulation. For $t \bar{t}, Z+$ jets and $W W$ production processes, which are the dominant backgrounds, the acceptance of the signal regions selection is estimated with MC simulation, while the normalisation is estimated in dedicated control regions. The MC samples used are the same as in Ref. [18].

The identification criteria for electrons, muons and jets follow the strategy defined in Appendix A: baseline electrons, which are used in the estimation of the fake-lepton background, are selected by applying the "medium" identification criteria. Signal electrons are identified using the "tight" criteria, and they are further required to be isolated. Signal muons correspond to baseline muons with an additional calorimeterand track-based isolation requirement applied. Jets that have $|\eta|<2.5$ and $p_{\mathrm{T}}>20 \mathrm{GeV}$ are used for the event selection, although all jets up to $|\eta|<4.5$ are retained for the computation of the missing transverse momentum.
Candidate stop production events, preselected by the same trigger and data quality requirements used in Ref. [18], are further required to contain one electron and one muon of opposite charge, with an invariant mass $m_{\ell \ell}>20 \mathrm{GeV}$. The leading (in $p_{\mathrm{T}}$ ) and next-to-leading leptons are required to have $p_{\mathrm{T}}>25 \mathrm{GeV}$ and $p_{\mathrm{T}}>20 \mathrm{GeV}$, respectively.

At this stage of the selection, the background is dominated by production of top-quark pairs and $Z \rightarrow \tau \tau$, followed by $W W$ and $W t$ production.

A requirement of $m_{\mathrm{T} 2}>20 \mathrm{GeV}$, where $m_{\mathrm{T} 2}$ is the stransverse mass of the two leptons, strongly reduces the $Z \rightarrow \tau \tau$ background, which is expected to have a kinematical end-point at $m_{\mathrm{T} 2}=m_{\tau}$. The ratio $R_{1}$ of the $E_{\mathrm{T}}^{\text {miss }}$ and the effective mass, defined as the scalar $p_{\mathrm{T}}$ sum of the $E_{\mathrm{T}}^{\mathrm{miss}}$, the leptons and the jets, is useful in suppressing the $t \bar{t}$ background, which is typically characterised by a larger hadronic activity than in signal events. The selection chosen is $R_{1}>0.3+m_{\text {eff }}$ (with $m_{\text {eff }}$ in $\mathrm{TeV}$ ).

After the above selections, the SM background is dominated by $W W$ production. Two differences between this process and the stop pair production signal are further exploited: firstly, the $W W$ production is dominated by quark-antiquark scattering, while stop pair production is mostly initiated by gluon-gluon processes, and secondly the stop pair production signal has four invisible (two neutralinos and two neutrinos) and two undetected (the two $b$-jets) objects, while the $W W$ process has only two. The first difference implies a higher longitudinal boost of the system emerging from the hard scattering in signal events than in background events. The variable

$\Delta X=2\left|\frac{\left(p_{z}\left(\ell_{1}\right)+p_{z}\left(\ell_{2}\right)\right)}{\sqrt{s}}\right|$

was defined in Ref. [119], and it is an estimator of the boost. The second difference implies a higher $E_{\mathrm{T}}^{\text {miss }}$ for signal events. This is exploited by making use of

$R_{2}=\frac{E_{\mathrm{T}}^{\text {miss }}}{E_{\mathrm{T}}^{\text {miss }}+p_{\mathrm{T}}\left(\ell_{1}\right)+p_{\mathrm{T}}\left(\ell_{2}\right)}$.

Finally, the variable $\cos \theta_{\mathrm{b}}$, the cosine of the angle between the direction of motion of one of the two leptons and the beam axis in the centre-of-mass frame of the two visible leptons [119], is sensitive to the spin of the produced particles, hence it provides additional rejection power against the $W W$ production process.

A set of seven signal regions were optimised for the discovery of stop pair production, with the stop decaying either as $\tilde{t}_{1} \rightarrow \tilde{\chi}_{1}^{ \pm} b$ with a branching ratio of $100 \%$ (assuming $m_{\tilde{t}_{1}}-m_{\tilde{\chi}_{1}^{ \pm}}<10 \mathrm{GeV}$ ), or as $\tilde{t}_{1} \rightarrow b W^{(*)} \tilde{\chi}_{1}^{0}$. The definitions of the signal regions are shown in Table 4. 
Table 4 Summary of signal regions used in the analysis. The upper part of the table shows the preselection requirements

\begin{tabular}{|c|c|c|c|c|c|c|c|}
\hline SR & WW-SR1 & WW-SR2 & WW-SR3 & WW-SR4 & WW-SR5 & WW-SR6 & WW-SR7 \\
\hline$p_{\mathrm{T}}\left(\ell_{1}\right)$ & $>25 \mathrm{GeV}$ & & & & & & \\
\hline$p_{\mathrm{T}}\left(\ell_{2}\right)$ & $>20 \mathrm{GeV}$ & & & & & & \\
\hline$R_{1}$ & $>0.3+m$ & & & & & & \\
\hline$m_{\mathrm{T} 2}$ & $>20 \mathrm{GeV}$ & & & & & & \\
\hline$\Delta X$ & $<0.02$ & & & & & & \\
\hline$R_{2}$ & $>0.5$ & & & & & & \\
\hline$\left|\cos \theta_{\mathrm{b}}\right|$ & $<0.8$ & $<0.8$ & $<0.8$ & - & - & $<0.8$ & - \\
\hline$m_{\mathrm{T} 2}$ & $<45 \mathrm{GeV}$ & $>25,<55 \mathrm{GeV}$ & - & $>70 \mathrm{GeV}$ & $>90 \mathrm{GeV}$ & $>25,<70 \mathrm{GeV}$ & $>80 \mathrm{GeV}$ \\
\hline
\end{tabular}

The background from non-prompt leptons originating from heavy-quark decays or from photon conversions in the signal regions, or from hadrons misidentified as leptons (collectively referred to as fake leptons in the following), is estimated as in Ref. [18].

Specific control regions, whose event yield is expected to be dominated by each of these production processes, are defined and included in the fit to constrain the normalisation parameters. The control region CRT for $t \bar{t}$ production is defined by changing the following selections with respect to the signal regions: $m_{\mathrm{T} 2}>35 \mathrm{GeV}, R_{1}<0.3$. Its purity is $92 \%$. The CR for $W W$ production (CRW) is defined by $m_{\mathrm{T} 2}>35 \mathrm{GeV}, \Delta X>0.04$, and has a purity of $72 \%$. Finally, the CR for $Z+$ jets (CRZ) is defined by $m_{\mathrm{T} 2}<20$ $\mathrm{GeV}, 30 \mathrm{GeV}<m_{\ell \ell}<80 \mathrm{GeV}$, with a purity of $86 \%$. The normalisation factors of the $W W, t \bar{t}, Z+$ jets production processes $\left(\mu_{W W}, \mu_{t \bar{t}}\right.$ and $\mu_{Z}$ respectively) are determined by a combined profile likelihood fit. When testing the signalplus-background hypothesis for rejection, the fit takes automatically into account the signal contamination in the control regions. For signal scenarios considering light $\left(m_{\tilde{t}_{1}}<150\right.$ $\mathrm{GeV}$ ) stops decaying through $\tilde{t}_{1} \rightarrow b W^{(*)} \tilde{\chi}_{1}^{0}$, the signal contamination becomes so large that $\mu_{W W}$ becomes unrealistically low. For such cases the fit is performed excluding CRW and taking the normalisation of the $W W$ background from MC simulation.

Systematic uncertainties, affecting both the modelling of the detector response (detector-related systematic uncertainties) and the theoretical prediction of the cross sections and acceptances of the background processes (theory-related systematic uncertainties) affect the predicted rates in the signal regions. Their classification and estimation follows closely those defined in Ref. [18]. A few differences, discussed in the following, exist on the estimation of the theory-related uncertainties. The total uncertainty on the yield of the $W W$ production process is composed of three terms: the uncertainty on the NLO hard-scattering calculation is taken to be the difference between the prediction of POWHEG and aMC@NLO both using PYTHIA for the parton shower; the uncertainty addressing the choice of the parton-shower model
Table 5 Normalisation factors for the $t \bar{t}, W W$ and $Z+$ jets background processes obtained by the combined fit to the control region yields. The uncertainties include systematic and statistical uncertainties

\begin{tabular}{ll}
\hline Normalisation factor & Value \\
\hline$\mu_{t \bar{t}}$ & $0.94 \pm 0.05$ \\
$\mu_{W W}$ & $1.01 \pm 0.11$ \\
$\mu_{Z}$ & $0.95 \pm 0.62$ \\
\hline
\end{tabular}

is estimated as the difference of the aMC@NLO predictions showered either with HERWIG or PYTHIA; the uncertainty due to the choice of the renormalisation and factorisation scale is evaluated by changing the scales independently by a factor of two or one-half and taking the maximum difference. The estimated relative uncertainties on the signal region yields are about $6 \%$ in SR1-SR4 and SR6; $11 \%$ in SR7 and $29 \%$ in SR5. Similar comparisons performed on the $W Z$ and $Z Z$ process yield uncertainties ranging from 30 to $45 \%$ depending on the signal region considered. Additional systematic uncertainties are assigned to the small expected yields from $Z+$ jets production (80 \%), Wt (50-100\% depending on the SR considered), and non-prompt lepton background.

The values of the normalisation factors obtained when performing the fit to the control regions only are shown in Table 5.

The overall predictions of the fit are compared to the data in dedicated validation region that are kinematically close to the signal region. They are defined by applying the preselection requirements of Table 4 with the additional selections shown in Table 6 . The $m_{\mathrm{T} 2}$ distribution in WW-VR2 and WW-VRT is shown in Fig. 17.

For all signal regions, the expected background yield is dominated by production of $W W(35 \%$ in SR 1 to $66 \%$ in SR4). Other important background processes are $Z+$ jets in SR1 $(20 \%)$, non-prompt leptons in SR2 (12\%), $t \bar{t}$ in all other SR, with contributions of about $10 \%$. The distributions of $E_{\mathrm{T}}^{\mathrm{miss}}$ and $m_{\mathrm{T} 2}$ in the signal region WW-SR3 are shown in Fig. 18. 
Table 6 Summary of the validation regions used in the WW analysis. The preselection requirements of Table 4 are also applied in all three validation regions

\begin{tabular}{lll}
\hline WW-VR1 & WW-VR2 & WW-VRT \\
\hline- & - & $0.3<R_{1}<0.3+m_{\text {eff }}(\mathrm{TeV})$ \\
$0.02<|\Delta X|<0.04$ & $\Delta X<0.02$ & $\Delta X<0.02$ \\
$R_{2}>0.5$ & $R_{2}<0.5$ & $R_{2}>0.5$ \\
$\left|\cos \theta_{\mathrm{b}}\right|<0.8$ & $\left|\cos \theta_{b}\right|<0.8$ & $\left|\cos \theta_{b}\right|<0.8$ \\
\hline
\end{tabular}

Table 7 compares the predicted and observed numbers of events in each of the signal regions. No excess above the SM prediction is observed, hence the results are first used to derive model-independent $95 \% \mathrm{CL}$ exclusion limits on the minimum number of events beyond the Standard Model in the signal region assuming no signal contamination in the control regions, and then to extract limits on $\sigma_{\mathrm{vis}}=\sigma \times \epsilon \times \mathcal{A}$, where $\sigma$ is the cross section for non-SM processes, $\epsilon$ is the selection efficiency and $\mathcal{A}$ is the selection acceptance. These limits are also reported in Table 7. Finally, $95 \%$ CL exclusion limits are derived in specific supersymmetric models of direct pair production of stops. The first exclusion limit (Fig. 19a) is derived in a model where the stop is assumed to decay as $\tilde{t}_{1} \rightarrow b \tilde{\chi}_{1}^{ \pm}$with a branching ratio of $100 \%$, followed by the decay of the chargino into the neutralino, assumed to be the stable LSP, through $\tilde{\chi}_{1}^{ \pm} \rightarrow W^{(*)} \tilde{\chi}_{1}^{0}$. The chargino mass is assumed to satisfy the relation $m_{\tilde{\chi}_{1}^{ \pm}}=m_{\tilde{t}_{1}}-10 \mathrm{GeV}$, and the limit is derived in the $m_{\tilde{t}_{1}}-m_{\tilde{\chi}_{1}^{0}}$ plane. Stop masses up to about $250 \mathrm{GeV}$ are excluded, almost independently of

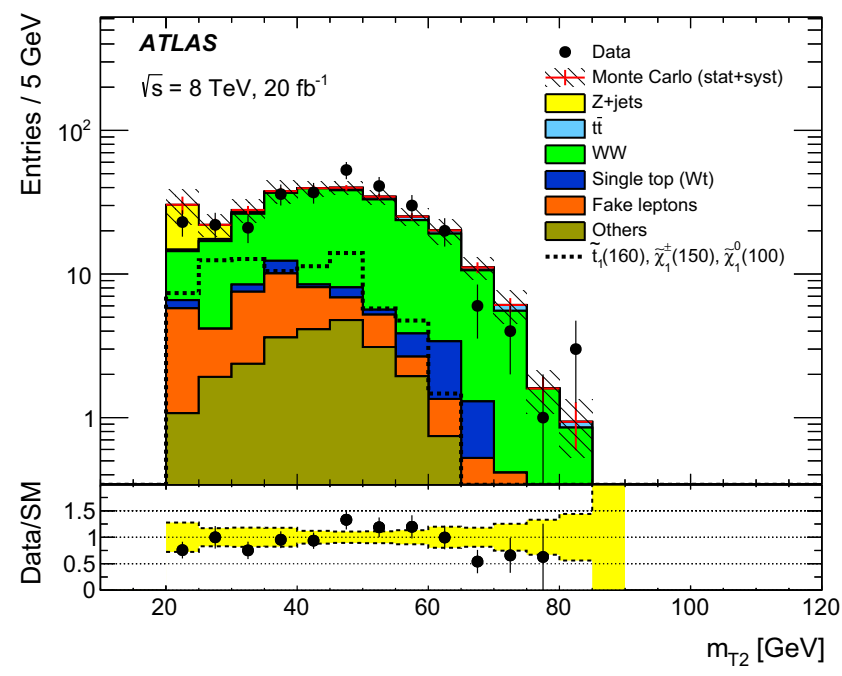

(a)

Fig. 17 Distribution of the stransverse mass $m_{\mathrm{T} 2}$ in the a WW-VR2 and $\mathbf{b}$ WW-VRT regions defined in the text. The contributions from all SM processes are shown as a histogram stack. The component labelled as "Fake leptons" includes the estimate of the background from nonprompt leptons. The expected signal for a model of stop pair production the neutralino mass. The second limit is derived in a model where the $\tilde{t}_{1}$ decays through its three-body or four-body decay (depending on its mass and on that of the neutralino) into $\tilde{t}_{1} \rightarrow b \ell \nu \tilde{\chi}_{1}^{0}$ with a branching ratio of $100 \%$, under the assumption that the decay happens through an off-shell top quark and an on- or off-shell $W$ boson. The limit is shown in Fig. 19b and fills a gap between the exclusions of the $2 \mathrm{~L}$ and $\mathrm{t} 1 \mathrm{~L}$ analyses.

\section{B.2.2 Final states containing two top quarks and a Higgs boson $(t 2 t 1 h)$}

If the lightest stop has a mass such that $\Delta m\left(\tilde{t}_{1}, \tilde{\chi}_{1}^{0}\right) \sim m_{t}$, the sensitivity of the searches for the production of a $\tilde{t}_{1}$ pair is greatly reduced. One of the approaches followed is to search for direct pair production of $\tilde{t}_{2}$ instead. This is the strategy used, for example, by the $2 \mathrm{t} 1 \mathrm{Z}$ analysis, whose signal regions were optimised to detect the decay of a pair-produced $\tilde{t}_{2}$ followed by the decay $\tilde{t}_{2} \rightarrow Z \tilde{t}_{1}$.

Inspired by the search for a SM Higgs boson produced in association with a top quark pair, a search was developed and optimised for the decay $\tilde{t}_{2} \rightarrow h \tilde{t}_{1}$, where the Higgs boson is assumed to have SM properties, and the $\tilde{t}_{1}$ is assumed to decay as $\tilde{t}_{1} \rightarrow t \tilde{\chi}_{1}^{0}$ with a BR of $100 \%$. The final state is hence characterised by a large jet multiplicity, by the presence of many $b$-jets from the top quark and Higgs boson decays and by $E_{\mathrm{T}}^{\text {miss }}$ associated with the presence of neutrinos from semileptonic decays of the top quark and of neutralinos.

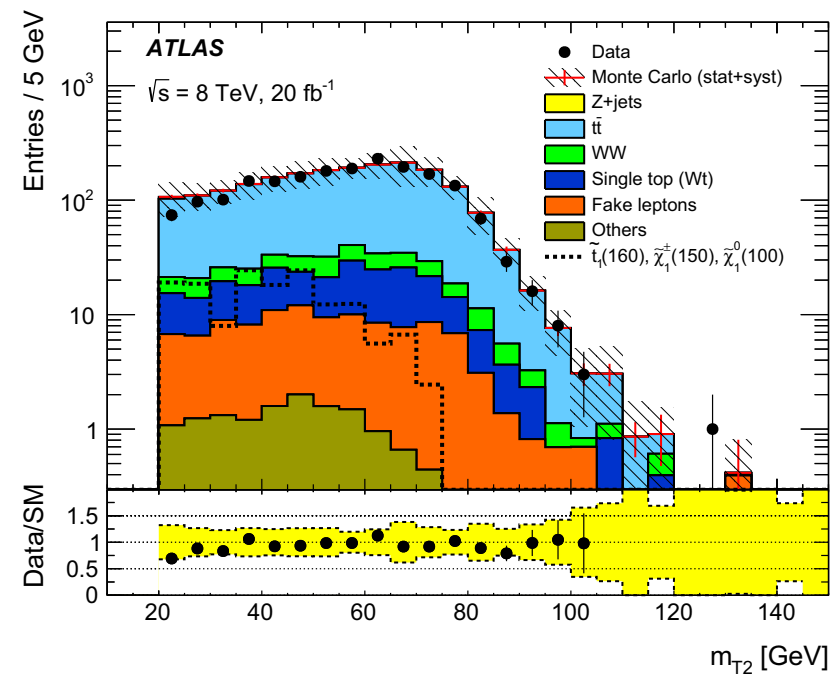

(b)

with the stop decaying as $\tilde{t}_{1} \rightarrow b \tilde{\chi}_{1}^{ \pm} \rightarrow b \ell^{ \pm} \nu \tilde{\chi}_{1}^{0}$ with $m_{\tilde{t}_{1}}=160 \mathrm{GeV}$, $m_{\tilde{\chi}_{1}^{ \pm}}=150 \mathrm{GeV}$ and $m_{\tilde{\chi}_{1}^{0}}=100 \mathrm{GeV}$ is also shown. The lower panels show the ratio between the data and the SM prediction; the yellow band includes statistical and systematic uncertainties on the SM prediction 


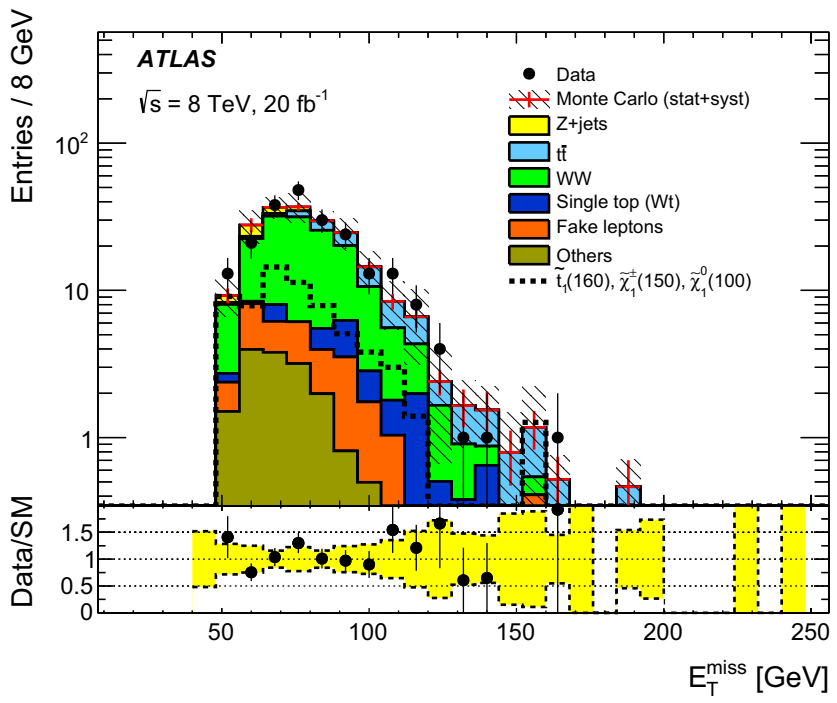

(a)

Fig. 18 Distribution of the a magnitude of missing transverse momentum $E_{\mathrm{T}}^{\text {miss }}$ and $\mathbf{b}$ stransverse mass $m_{\mathrm{T} 2}$ in WW-SR3. The contributions from all SM processes are shown as a histogram stack. The component labelled as "Fake leptons" includes the estimate of the background from non-prompt leptons. The expected signal for a model of stop pair

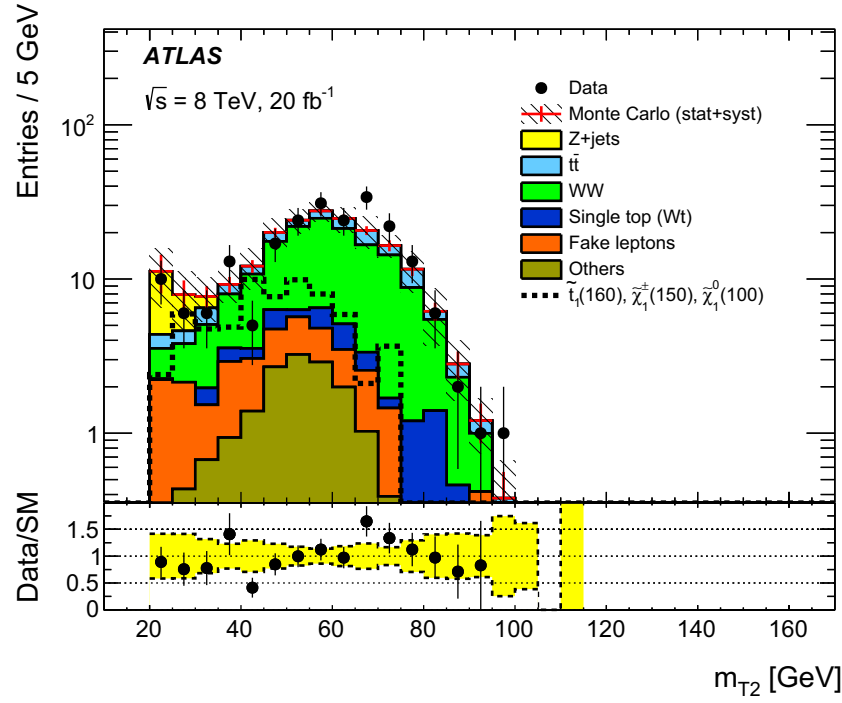

(b)

production with the stop decaying into $\tilde{t}_{1} \rightarrow b \tilde{\chi}_{1}^{ \pm} \rightarrow b \ell^{ \pm} v \tilde{\chi}_{1}^{0}$ with $m_{\tilde{t}_{1}}=160 \mathrm{GeV}, m_{\tilde{\chi}_{1}^{ \pm}}=150 \mathrm{GeV}$ and $m_{\tilde{\chi}_{1}^{0}}=100 \mathrm{GeV}$ is also shown. The lower panels show the ratio between the data and the SM prediction; the yellow band includes statistical and systematic uncertainties on the SM prediction
Table 7 Observed (Obs) and predicted (Exp) numbers of events in the signal regions of the WW analysis, together with the $95 \%$ CL upper limits on the observed and expected number of signal events ( $S_{\mathrm{obs}}^{95}$ and $S_{\mathrm{exp}}^{95}$, respectively), and on the visible cross section $\left(\langle\epsilon \sigma\rangle_{\mathrm{obs}}^{95}\right)$

\begin{tabular}{lrcccc}
\hline Signal channel & Obs & \multicolumn{1}{l}{ Exp } & \multicolumn{1}{c}{$S_{\text {obs }}^{95}$} & $S_{\text {exp }}^{95}$ & $\langle\epsilon \sigma\rangle_{\text {obs }}^{95}(\mathrm{fb})$ \\
\hline SR1 & 40 & $47 \pm 14$ & 22.6 & $25.2_{-4.3}^{+9.4}$ & 1.12 \\
SR2 & 71 & $80 \pm 13$ & 25.3 & $27.8_{-4.1}^{+11.5}$ & 1.24 \\
SR3 & 215 & $203 \pm 27$ & 48.4 & $46.6_{-6.9}^{+4.9}$ & 2.38 \\
SR4 & 88 & $81 \pm 11$ & 35.1 & $28.8_{-5.4}^{+11.0}$ & 1.73 \\
SR5 & 4 & $3.4 \pm 0.9$ & 6.2 & $5.7_{-1.4}^{+2.1}$ & 0.30 \\
SR6 & 160 & $154 \pm 19$ & 45.6 & $43.8_{-14.4}^{+19.3}$ & 2.25 \\
SR7 & 21 & $23 \pm 4$ & 12.4 & $13.4_{-3.4}^{+4.8}$ & 0.61 \\
\hline
\end{tabular}

The selection of electrons, muons, jets and $b$-jets follows the principles outlined in Appendix A. The specific choices made for the $p_{\mathrm{T}}$ and pseudorapidity thresholds and working points of the final-state objects, as well as the trigger selection, are the same as those in Ref. [120]. The selection requires the presence of exactly one electron or muon with $p_{\mathrm{T}}>25 \mathrm{GeV}, E_{\mathrm{T}}^{\text {miss }}>50 \mathrm{GeV}$, at least six jets with $p_{\mathrm{T}}>25$ $\mathrm{GeV}$ and $|\eta|<2.5$, of which at least two are required to be $b$-tagged. The working point chosen for the $b$-tagging is such that the efficiency to tag $b$-jets (evaluated on a MC sample of $t \bar{t}$ production) is about $70 \%$.

The modelling of the production of $t \bar{t}$ pairs in association with heavy flavour $(t \bar{t}+\mathrm{HF})$ is of key relevance in this analysis. A detailed categorisation of $t \bar{t}+\mathrm{HF}$ is made for the purpose of comparisons with different generators and of the propagation of systematic uncertainties on the different heavy-flavour components. The categorisation is also used to reweight the different flavour components of the $t \bar{t}+$ jets background to obtain a better modelling. These categorisation and reweighting procedures are discussed in detail in Ref. [120]. In particular, the $t \bar{t}+b \bar{b}$ component, which is simulated with POWHEG, is reweighted to a full NLO calculation [121] performed in SHERPA 1.4.1+OpenLoops $[122,123]$. The reweighting is done at generator level using a number of kinematic variables such as the top quark $p_{\mathrm{T}}, t \bar{t}$ system $p_{\mathrm{T}}, \Delta R$ and $p_{\mathrm{T}}$ of the dijet system not coming from the top-quark decay. A different reweighting is applied to the $t \bar{t}+c \bar{c}$ and $t \bar{t}+$ lightjets components, which is based on the ratio of the differential cross sections at $\sqrt{s}=7 \mathrm{TeV}$ obtained in data and simulation as a function of the top quark $p_{\mathrm{T}}$ and $t \bar{t}$ system $p_{\mathrm{T}}[124]$.

The selected events are categorised into different channels, depending on the number of $b$-tagged jets (two, 


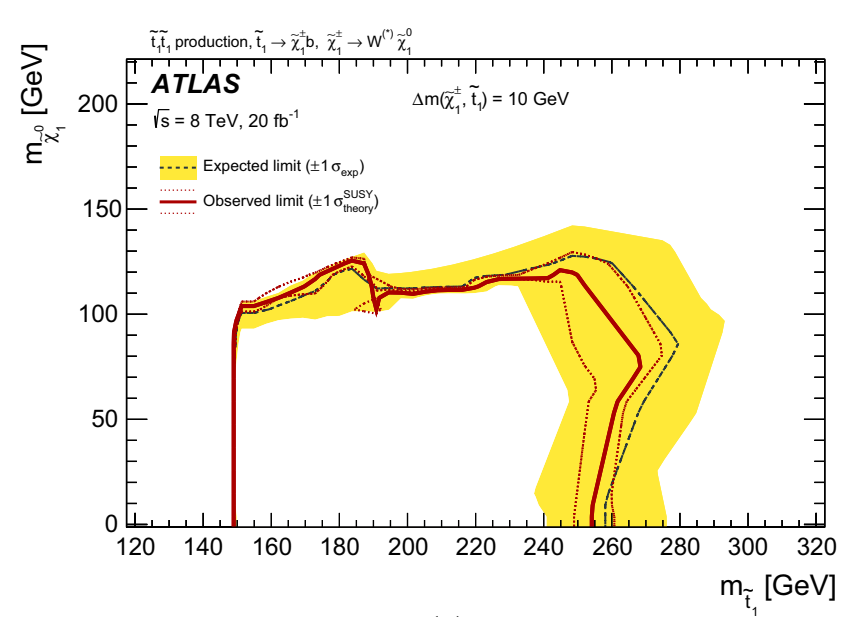

(a)

Fig. 19 Exclusion limits at $95 \% \mathrm{CL}$ in the scenario where both pair-produced stop decay exclusively via a $\tilde{t}_{1} \rightarrow b \tilde{\chi}_{1}^{ \pm}$followed by $\tilde{\chi}_{1}^{ \pm} \rightarrow W \tilde{\chi}_{1}^{0}$, with $\Delta m\left(\tilde{t}_{1}, \tilde{\chi}_{1}^{ \pm}\right)=10 \mathrm{GeV}$, and $\mathbf{b}$ three-body or fourbody decay (depending on the neutralino and stop mass). The black dashed line indicates the expected limit, and the yellow band indicates the $\pm 1 \sigma$ uncertainties, which include all uncertainties except the

three or at least four). The channel with at least four $b$ jets has the largest signal-to-background ratio. The channels with two and three $b$-tagged jets are used to calibrate the $t \bar{t}+$ jets background prediction and constrain the associated systematic uncertainties, which, in the channel with at least four $b$-tagged jets, are dominated by the $b$ tagging, jet energy scale, and $t \bar{t}+$ jets heavy-flavour content uncertainties.

For a given $b$-tag multiplicity, events are further categorised depending on the value of the transverse mass $m_{\mathrm{T}}$ of the lepton and the missing transverse momentum. A "low- $m_{\mathrm{T}}$ " ("high- $m_{\mathrm{T}}$ ") region is defined by the requirement $m_{\mathrm{T}}<120 \mathrm{GeV}\left(m_{\mathrm{T}}>120 \mathrm{GeV}\right)$.

The final discriminating variable used is $H_{\mathrm{T}}^{\text {nolep }}$, defined as the scalar sum of $E_{\mathrm{T}}^{\text {miss }}$ and the transverse momenta of all selected jets. The signal is searched for by performing a binned likelihood fit to the $H_{\mathrm{T}}^{\text {nolep }}$ distribution simultaneously in the six channels defined (low/high- $m_{\mathrm{T}}$ for three bins in $b$-tagged jet multiplicity). The binning used for the $H_{\mathrm{T}}^{\text {nolep }}$ distributions is that used in Fig. 20, where the background estimate both before and after the fit is compared to the data in the high- $m_{\mathrm{T}}$ region. The dominant post-fit uncertainties are those on the absolute normalisation of the $t \bar{t}+b \bar{b}$ and $t \bar{t}+c \bar{c}$ processes.

The full list of detector systematic uncertainties considered, discussed in detail in Ref. [120], includes, beside a total uncertainty of $2.8 \%$ on the integrated luminosity, systematic uncertainties on the identification efficiency and energy scale uncertainty of the leptons, reconstruction efficiency and energy scale and resolution uncertainties for jets, $b$-tagging

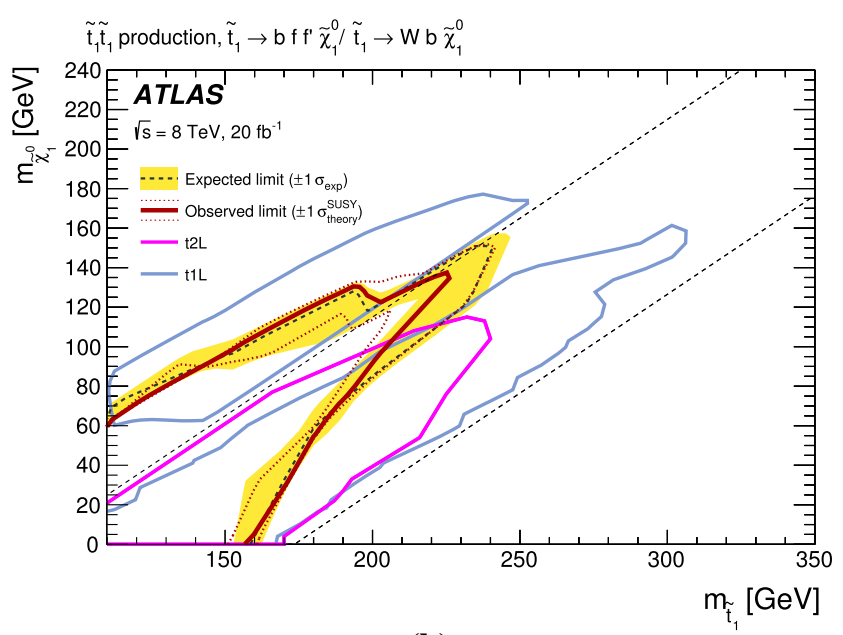

(b)

theoretical uncertainties in the signal. The red solid line indicates the observed limit, and the red dotted lines indicate the sensitivity to $\pm 1 \sigma$ variations of the signal theoretical uncertainties. For $\mathbf{b}$, the observed limits achieved by the $11 \mathrm{~L}$ and $\mathrm{t} 2 \mathrm{~L}$ analyses are also shown, and the straight dashed lines correspond to $\Delta m\left(\tilde{t}_{1}, \tilde{\chi}_{1}^{0}\right)=m_{W}+m_{b}$ and $\Delta m\left(\tilde{t}_{1}, \tilde{\chi}_{1}^{0}\right)=m_{t}$

efficiency and mis-tag rate uncertainties. Further modelling uncertainties are considered, which include, beside production cross-section uncertainties for $W / Z+$ jets, single top and $t \bar{t}$, dedicated uncertainties on the NLO calculation of the $t \bar{t}+b \bar{b}$ process and on the modelling of the $t \bar{t}+c \bar{c}$ component.

No significant excess above the expected background is observed, hence $95 \%$ CL limits are derived in a model where $\tilde{t}_{2}$ production is assumed, followed by the decay $\tilde{t}_{2} \rightarrow \tilde{t}_{1} h$ (with a branching ratio of $100 \%$ ) and $\tilde{t}_{1} \rightarrow t \tilde{\chi}_{1}^{0}$ (again with a branching fraction of $100 \%$ ). ${ }^{14}$ The limit is derived as a function of the $\tilde{t}_{2}$ and $\tilde{\chi}_{1}^{0}$ masses, under the assumption that $\Delta m\left(\tilde{t}_{1}, \tilde{\chi}_{1}^{0}\right)=180 \mathrm{GeV}$, and it is presented in Sect. 4.3.

\section{B.2.3 Final states containing two b-jets, a charged lepton, and missing transverse momentum ( $t b$ )}

Several phenomenological models, where both $\tilde{\chi}_{1}^{ \pm}$and $\tilde{\chi}_{1}^{0}$ are lighter than the stop (or the sbottom), allow for the $\tilde{t}_{1} \rightarrow t \tilde{\chi}_{1}^{0}$, $\tilde{t}_{1} \rightarrow b \tilde{\chi}_{1}^{ \pm}$and $\tilde{b}_{1} \rightarrow b \tilde{\chi}_{1}^{0}, \tilde{b}_{1} \rightarrow t \tilde{\chi}_{1}^{ \pm}$decay channels to be open with competing branching ratios. Naturalness arguments require the higgsino mass parameter $\mu$ to be smaller than a few hundred $\mathrm{GeV}$, while they impose virtually no constraint on the bino and wino mass parameters $M_{1}$ and $M_{2}$. If $\mu \ll M_{1}, M_{2}$, then the lightest chargino and neutralino masses are both of the order of $\mu$ and hence $\Delta m\left(\tilde{\chi}_{1}^{ \pm}, \tilde{\chi}_{1}^{0}\right)$ is small. Therefore, pair production of stops can lead to

\footnotetext{
${ }^{14}$ Production of $\tilde{t}_{1}$ pairs is also included in the simplified models. The acceptance of the selection for such events is very small. Nevertheless, this component is considered as signal in the statistical analysis.
} 
Fig. 20 Comparison between data and prediction for the distribution of $H_{\mathrm{T}}^{\text {nolep }}$, defined as the scalar sum of the missing transverse momentum and the transverse momenta of all selected jets, in the high- $m_{\mathrm{T}}$ channels considered: (top) two $b$-tagged jets, (middle) three $b$-tagged jets and (bottom) four $b$-tagged jets, (left) before and (right) after the combined fit to data under the background-only hypothesis. The expected signal contributions from $\tilde{t}_{1}$ and $\tilde{t}_{2}$ pair production, assuming $m_{\tilde{t}_{2}}=500 \mathrm{GeV}, m_{\tilde{t}_{1}}=300$ $\mathrm{GeV}, m_{\tilde{\chi}_{1}^{0}}=120 \mathrm{GeV}$ and a branching ratio of $100 \%$ for $\tilde{t}_{2} \rightarrow h \tilde{t}_{1}$ are also shown added to the stack (red histograms, in dark red the contribution from direct $\tilde{t}_{1}$ pair production). The bottom panel displays the ratio of the data to the total background prediction. The hashed area represents the statistical and systematics uncertainty on the background

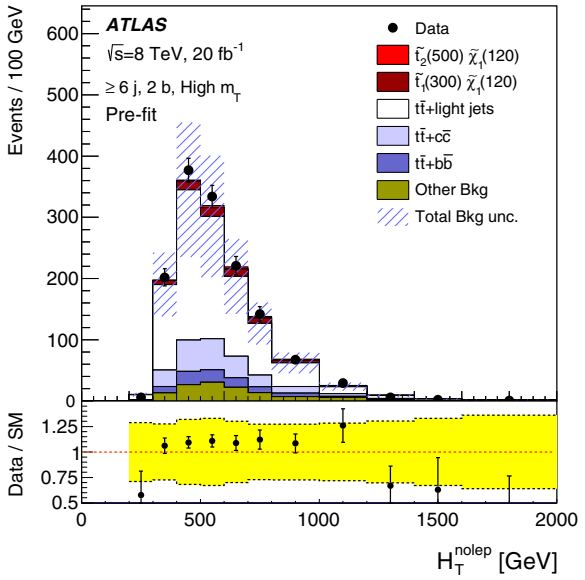

(a)

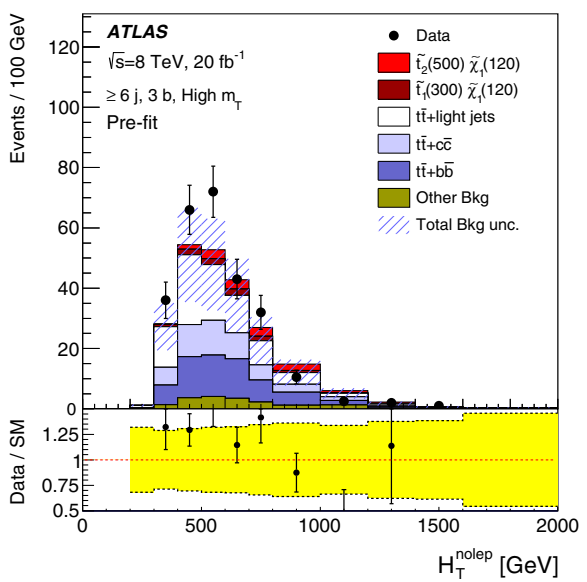

(c)

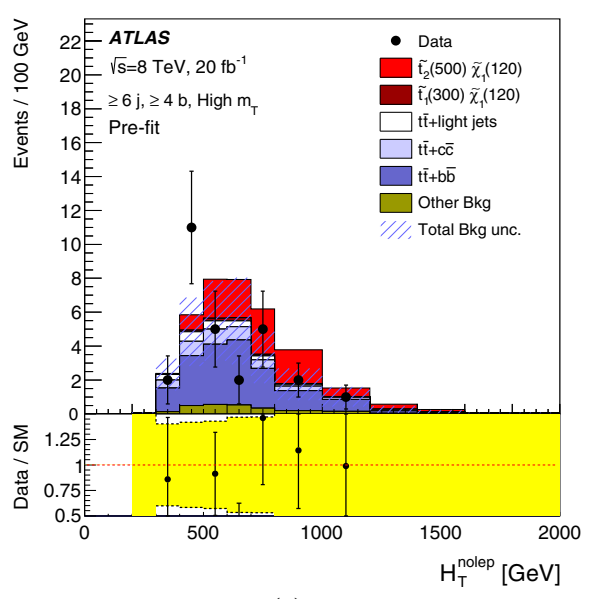

(e)

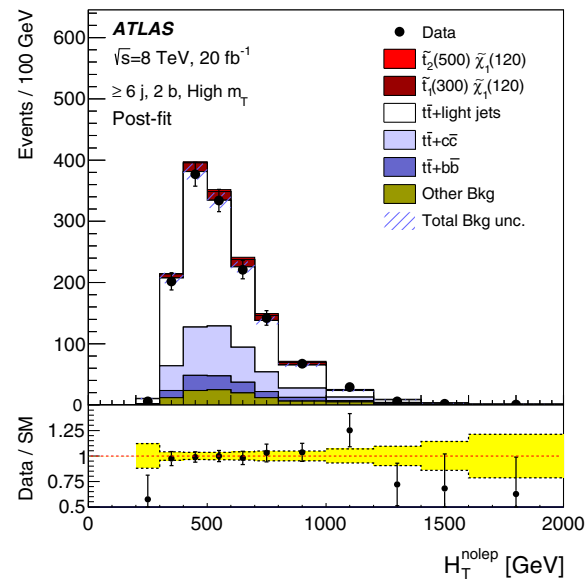

(b)

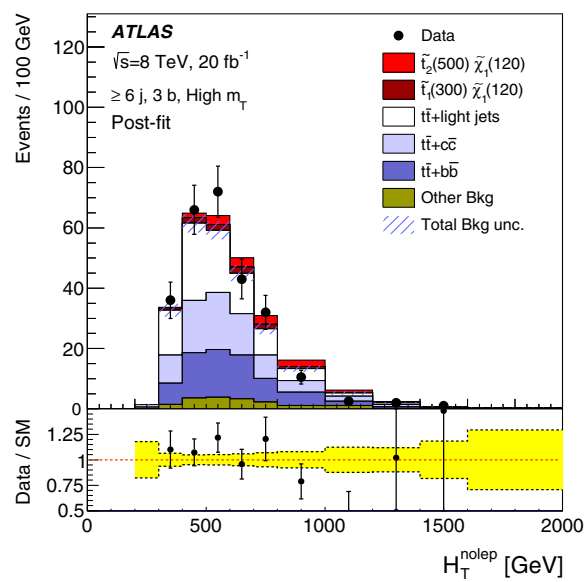

(d)

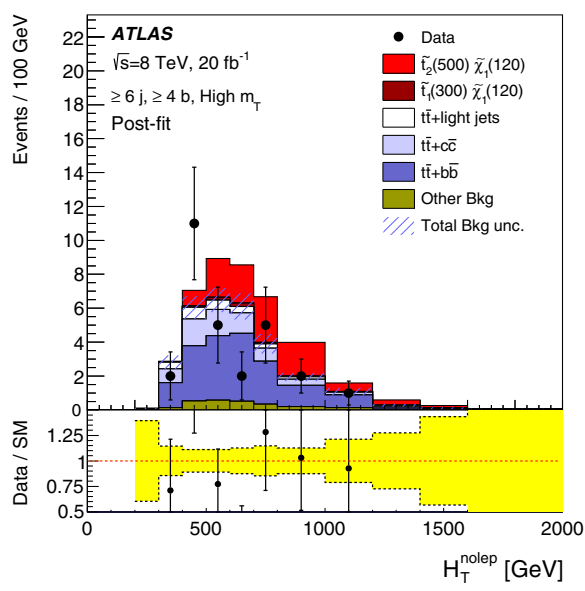

(f) $\tilde{t}_{1} \tilde{t}_{1} \rightarrow t \tilde{\chi}_{1}^{0} b \tilde{\chi}_{1}^{ \pm} \rightarrow t b \tilde{\chi}_{1}^{0} \tilde{\chi}_{1}^{0} f f^{\prime}$, where $f$ and $f^{\prime}$ represents low- $p_{\mathrm{T}}$ fermions emitted through $\tilde{\chi}_{1}^{ \pm} \rightarrow f f^{\prime} \tilde{\chi}_{1}^{0}$. Assuming both $f$ and $f^{\prime}$ are too soft to be detected, the final state is characterised by the presence of a top quark, a bottom quark, and neutralinos escaping the detector. Similarly, $\tilde{b}_{1}$ pair production can lead to the same final state. Dedicated SRs are defined that target this topology, which is not well covered by the $\mathrm{t} 0 \mathrm{~L}$ and $\mathrm{t} 1 \mathrm{~L}$ signal regions aimed at final states containing $t \bar{t} E_{\mathrm{T}}^{\text {miss }}$ and the b0L signal regions targeting $b \bar{b} E_{\mathrm{T}}^{\text {miss }}$ final states.

Both the leptonic and hadronic decays of the top quark have been studied, and the leptonic channel was found to give a better sensitivity to the signal models of interest. The dominant SM background processes in the signal regions are 
Table 8 Summary of signal regions used by the tb analysis

\begin{tabular}{|c|c|c|c|c|}
\hline SR & tb-SRIn1 & tb-SRIn2 & tb-SRIn3 & tb-SREx 1 \\
\hline$b$-jets & \multicolumn{4}{|c|}{$2 b$-jets; $p_{\mathrm{T}}>25 \mathrm{GeV}$} \\
\hline \multirow[t]{2}{*}{1 lepton } & \multicolumn{4}{|c|}{$p_{\mathrm{T}}>25 \mathrm{GeV}$} \\
\hline & \multicolumn{4}{|c|}{$|\eta|<2.5(2.47)$ for $\mu(e)$} \\
\hline$E_{\mathrm{T}}^{\text {miss }}(\mathrm{GeV})$ & $>200$ & $>120$ & $>220$ & $>160$ \\
\hline$m_{\mathrm{T}}(\mathrm{GeV})$ & $>140$ & $>140$ & $>180$ & $>120$ \\
\hline$m_{\mathrm{eff}}(\mathrm{GeV})$ & $>300$ & $>450$ & $>650$ & $>300$ \\
\hline$a m_{\mathrm{T} 2}(\mathrm{GeV})$ & $>180$ & $>200$ & $>180$ & $>180$ \\
\hline$m_{b \ell}^{\min }(\mathrm{GeV})$ & \multicolumn{4}{|l|}{$<170$} \\
\hline$\Delta \phi_{\min }^{b}$ & \multicolumn{4}{|l|}{$>0.4$} \\
\hline$E_{\mathrm{T}}^{\text {miss }}$ significance $\left(\mathrm{GeV}^{1 / 2}\right)$ & $>8$ & $>12$ & $>5$ & $>10$ \\
\hline$N_{\text {xjets }}$ & - & - & - & $<2$ \\
\hline
\end{tabular}

semileptonic $t \bar{t}$ and single top production. The SM background is evaluated using a combination of Monte Carlo and partially data-driven techniques.

Events are selected online by a trigger requiring the presence of one electron or muon. The online selection thresholds are such that the plateau efficiency is reached for lepton transverse momenta of $25 \mathrm{GeV}$.

The identification criteria for electrons, muons, jets and $E_{\mathrm{T}}^{\text {miss }}$ follow the principles outlined in Appendix A. In particular, electrons and muons are required to be isolated: the scalar $p_{\mathrm{T}}$ sum of tracks in a cone $\Delta R=0.2$ around the electron (muon) is required to be smaller than $10 \%$ of the electron transverse momentum $(1.2 \mathrm{GeV})$. The electron or muon track is excluded from the sum. The $b$-tagging algorithm is used at an operating point with $70 \%$ efficiency in simulated top-quark pair production events. Signal regions are defined as detailed in Table 8 , requiring one and only one electron or muon, two $b$-tagged jets and a large $E_{\mathrm{T}}^{\text {miss }}$. Three of the SRs, labelled tb-SRin have no additional jet veto applied, while one of them (tb-SREx1) has a veto requirement on the number of jets ( $\left.N_{\text {xjets }}\right)$ with $p_{\mathrm{T}}>50 \mathrm{GeV}$ in addition to the two leading $b$-tagged jets. The final SR optimisation is performed by using selections on the momenta of the objects, the $m_{\mathrm{T}}$ and the $m_{\mathrm{eff}}$ variables. In addition, the following kinematic variables are used in the event selection:

- $\Delta \phi_{\min }^{b}$ : the minimum azimuthal distance between the closest $b$-tagged jet and the $E_{\mathrm{T}}^{\text {miss }}$. This variable is used to remove multijet backgrounds with a cut of $\Delta \phi_{\min }^{b}>0.4$.

- $m_{\text {eff }}$ : the scalar sum of the $p_{\mathrm{T}}$ of the two $b$-jets (with $p_{\mathrm{T}}>25 \mathrm{GeV}$ and $|\eta|<2.8$ ) plus at most one light jet (with $p_{\mathrm{T}}>25 \mathrm{GeV}$ and $|\eta|<2.5$ ) and the $E_{\mathrm{T}}^{\text {miss }}$. The number of light jets, $n$, included in this sum depends on the signal region under study, although $n=1$ was mostly used.
- $E_{\mathrm{T}}^{\text {miss }}$ significance: the ratio of the $E_{\mathrm{T}}^{\text {miss }}$ to the square root of $H_{\mathrm{T}}$, which is the scalar sum of the $p_{\mathrm{T}}$ of the two $b$-jets plus one light jet with $p_{\mathrm{T}}>25 \mathrm{GeV}$ and $|\eta|<2.8$.

$-m_{\mathrm{T}}$ : the transverse mass of the lepton and the missing transverse momentum vector.

$-m_{b b}$ : the invariant mass of the two $b$-tagged jets.

- $m_{b \ell}$ : the invariant mass of a $b$-tagged jet and the charged lepton. This variable is bounded from above at $m_{t}$ in $t \bar{t}$ production events. Since two jets are $b$-tagged the variables $m_{b \ell}$ (1) and $m_{b \ell}$ (2) are defined to indicate the invariant mass constructed with the leading and subleading $b$-jet respectively. The variable $m_{b \ell}^{\min }$ is also defined to indicate the minimum between $m_{b \ell}$ (1) and $m_{b \ell}$ (2).

- $a m_{\mathrm{T} 2}$ : the asymmetric stransverse mass [108] is a kinematic variable which can be used to separate processes in which two decays giving missing transverse momentum occur. It is defined as follows:

$$
\begin{aligned}
\operatorname{am}_{\mathrm{T} 2}^{2}(\chi)= & \min _{\mathbf{q}_{\mathrm{T}}^{(1)}+\mathbf{q}_{\mathrm{T}}^{(2)}=\mathbf{p}_{\mathrm{T}}} \times\left[\operatorname { m a x } \left\{m_{\mathrm{T}}^{2}\left(\mathbf{p}_{\mathrm{T}}\left(v_{1}\right), \mathbf{q}_{\mathrm{T}}^{(1)} ; \chi\right),\right.\right. \\
& \left.\left.m_{\mathrm{T}}^{2}\left(\mathbf{p}_{\mathrm{T}}\left(v_{2}\right), \mathbf{q}_{\mathrm{T}}^{(2)} ; \chi\right)\right\}\right]
\end{aligned}
$$

where $\mathbf{p}_{\mathrm{T}}\left(v_{i}\right)$ are reconstructed transverse momentum vectors, $\mathbf{q}_{\mathrm{T}}^{(i)}$ represent the missing transverse momenta from the two decays, with a total missing transverse momentum, $\not p_{\mathrm{T}}$, and $\chi$ is a free parameter representing the unknown neutralino mass, which is assumed to be zero in the calculation. The $a m_{\mathrm{T} 2}$ variable is calculated with different choices for $\mathbf{p}_{\mathrm{T}}\left(v_{1}\right)$ and $\mathbf{p}_{\mathrm{T}}\left(v_{2}\right)$, depending on the value of $m_{b \ell}(n)(n=1,2)$, the invariant mass of the $n^{\text {th }} b$-tagged jet $b_{n}$ and the lepton:

- if $m_{b \ell}(i)<170 \mathrm{GeV}$ and $m_{b \ell}(j)>170 \mathrm{GeV}$, then $a m_{\mathrm{T} 2}$ is calculated with $v_{1}=b_{i}+\ell$ and $v_{2}=b_{j}$;

- if $m_{b \ell}(1)<170 \mathrm{GeV}$ and $m_{b \ell}(2)<170 \mathrm{GeV}$, then $a m_{\mathrm{T} 2}$ is evaluated using the two possible combinations for $v_{1}$ and $v_{2}$, and the minimum is used; 
- it both $m_{b \ell}(1)>170 \mathrm{GeV}$ and $m_{b \ell}(2)>170 \mathrm{GeV}$ the event is rejected.

The case of both $m_{b \ell}$ (1) and $m_{b \ell}$ (2) exceeding $170 \mathrm{GeV}$ is irrelevant: only events with the minimum value of $m_{b \ell}$ smaller than $170 \mathrm{GeV}$ populate the control, validation and signal regions.

The optimisation is carried out using both a pMSSM signal model and simplified models where $\Delta m\left(\tilde{\chi}_{1}^{ \pm}, \tilde{\chi}_{1}^{0}\right)=5$ or $10 \mathrm{GeV}$. In the case of the pMSSM model, additional non- $b$ tagged jets are expected in the final state via the production of other SUSY particles, hence the optimisation points to SRs with no requirement on the $N_{\text {xjets }}$ variable (tb-SRIn). In the case of the simplified models, additional jets come only from initial- or final-state radiation, and as a consequence a strict selection on $N_{\text {xjets }}$ is applied as in the selection tb-SREx1.

The main SM backgrounds are top-pair production, $W$ production in association with heavy-flavour jets and singletop production. The MC cross section is used to normalise the single-top background and all the other minor SM backgrounds, such as $Z+$ jets, diboson production, $t \bar{t}+W$ and $t \bar{t}+Z$. The normalisation factors of the $t \bar{t}$ and $W+$ jets backgrounds are determined by a combined profile-likelihood fit. Specific control regions, whose event yield is expected to be dominated by each of these production processes, are defined and included in the fit to constrain the normalisation parameters. The $t \bar{t}$ control regions (CRT) are defined by inverting the selection on $a m_{\mathrm{T} 2}$, requiring $a m_{\mathrm{T} 2}<160$ (180) GeV for the inclusive (exclusive) SRs. The purity of the $t \bar{t}$ process in the CRTs is in excess of $95 \%$. The $W+$ jets control regions (CRW) are defined by requiring $m_{\mathrm{T}}<120 \mathrm{GeV}$. For the control regions corresponding to the tb-SRIn, events with one $b$-tagged jet are included in the CRW. Top quark pair production dominates the CRWs, with a $W+$ jets purity of $30 \%$ or better. The normalisation factors $\mu_{W}$ and $\mu_{t \bar{t}}$ are presented in Table 9. The background model is then validated using validation regions, where little signal contamination is expected.

The distributions of the variable $a m_{\mathrm{T} 2}$ in the four SRs are shown in Fig. 21 together with the expected distribution from some of the signal models used to optimise the analysis. Table 10 compares the predicted and observed numbers of events in each of the signal regions. No excess above the

Table 9 Background scale factors for the $t \bar{t}$ and $W$ samples, as obtained by the background fit. The errors include both the statistical and systematics uncertainties

\begin{tabular}{lllll}
\hline Norm. factor & SRinA & SRinB & SRinC & SRexA \\
\hline$\mu_{t \bar{t}}$ & $1.06 \pm 0.07$ & $1.12 \pm 0.09$ & $0.94 \pm 0.21$ & $1.06 \pm 0.07$ \\
$\mu_{W}$ & $0.92 \pm 0.20$ & $0.61 \pm 0.23$ & $0.93 \pm 0.27$ & $1.10 \pm 0.34$ \\
\hline
\end{tabular}

SM prediction is observed, hence the results are first used to derive model-independent $95 \%$ CL exclusion limits on the number of events beyond the Standard Model in the signal region, and then to extract limits on $\sigma_{\mathrm{vis}}=\sigma \times \epsilon \times \mathcal{A}$, where $\sigma$ is the cross section for non-SM processes, $\epsilon$ is the selection efficiency and $\mathcal{A}$ is the selection acceptance. All these limits are also reported in Table 10.

Since the number of events observed agrees with the SM predictions, $95 \%$ CL exclusion limits are derived in specific supersymmetric models of direct pair production of stops. Simplified models were simulated with the two decays $\tilde{t}_{1} \rightarrow t \tilde{\chi}_{1}^{0}, \tilde{t}_{1} \rightarrow b \tilde{\chi}_{1}^{ \pm}$each having a $50 \%$ BR for values of $\Delta m\left(\tilde{\chi}_{1}^{ \pm}, \tilde{\chi}_{1}^{0}\right)=5,20 \mathrm{GeV}$. Furthermore, by using a weighted combination of these simplified models with models corresponding to a $100 \% \mathrm{BR}$ in either $\tilde{t}_{1} \rightarrow t \tilde{\chi}_{1}^{0}$ or $\tilde{t}_{1} \rightarrow b \tilde{\chi}_{1}^{ \pm}$, limits can be obtained for any value of the stop BR. Figure 22 shows the exclusion limits for $\operatorname{BR}\left(\tilde{t}_{1} \rightarrow t \tilde{\chi}_{1}^{0}\right)=25,50$ and $75 \%$ for the two values of $\Delta m\left(\tilde{\chi}_{1}^{ \pm}, \tilde{\chi}_{1}^{0}\right)$ considered.

Finally, $95 \%$ CL exclusion limits are also derived for a natural pMSSM model and are presented in Fig. 23

\section{Further details of the statistical combination of the $\mathrm{t} 0 \mathrm{~L}$ and $\mathrm{t} 1 \mathrm{~L}$ signal regions}

This section provides additional details on the combination of the t0L and $11 \mathrm{~L}$ signal regions targeting scenarios in which the stop decays into either $\tilde{t}_{1} \rightarrow t \tilde{\chi}_{1}^{0}$ or the mixed case where $\tilde{t}_{1} \rightarrow t \tilde{\chi}_{1}^{0}$ and $\tilde{t}_{1} \rightarrow b \tilde{\chi}_{1}^{ \pm}$are both allowed, as discussed in Sect. 4.1

The statistical combination of the two analyses is performed by running the combined fit simultaneously on the control and signal regions of the two analyses. The detector systematic uncertainties are treated as correlated by using, for each of the uncertainties considered, a single nuisance parameter. The supersymmetric signal parameter strength used is the same for the two analyses, while the normalisation parameters for the background processes are kept independent in each analysis. ${ }^{15}$ The nuisance parameters associated with modelling uncertainties of the various processes are also kept independent.

The control regions of the two analyses are not mutually exclusive: the events that belong to both a $\mathrm{CR}$ of $\mathrm{t} 0 \mathrm{~L}$ and one of $11 \mathrm{~L}$ are, at most about $2 \%$ of the total number of events of the tOL CR. The strategy adopted is to remove them from the corresponding tOL CR for the combination. It has been verified that such removal does not affect the individual results of the t0L analysis.

\footnotetext{
15 The choice is motivated by the fact that the phase-space regions in which the two analyses determine the normalisation parameters of the $t \bar{t}, Z+$ jets and $W+$ jets (for $\mathrm{t} 0 \mathrm{~L}$ ) and $t \bar{t}$ and $W+$ jets (for $\mathrm{t} 1 \mathrm{~L}$ ) are characterised by different kinematic selections and jet multiplicities.
} 


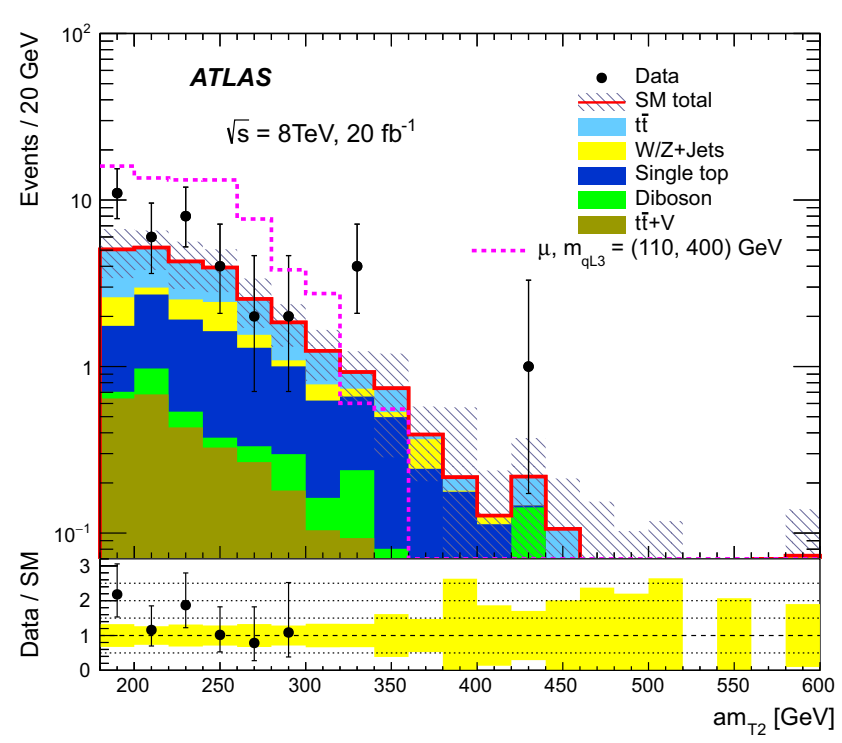

(a)

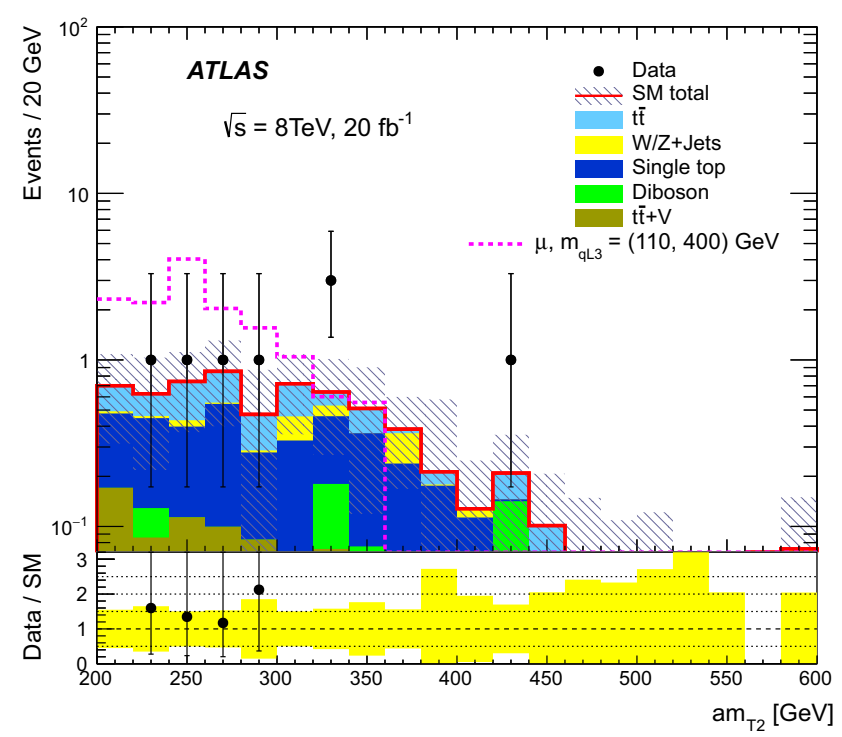

(c)

Fig. 21 Distribution of the asymmetric stransverse mass $a m_{\mathrm{T} 2}$ in the a SRinA, b SRinB (top right), c SRinC and d SRexA defined in the text. The contributions from all SM processes are shown as a histogram stack. The contribution from signal points studied by this analysis are

Table 10 Observed (Obs) and predicted (Exp) numbers of events in the signal regions of the tb analysis, together with the $95 \%$ CL upper limits on the observed and expected number of signal events ( $S_{\mathrm{obs}}^{95}$ and $S_{\exp }^{95}$ respectively), and on the visible cross section $\left(\langle\epsilon \sigma\rangle_{\mathrm{obs}}^{95}\right)$

\begin{tabular}{lcccll}
\hline Signal channel & Obs & Exp & $S_{\text {obs }}^{95}$ & $S_{\text {exp }}^{95}$ & $\langle\epsilon \sigma\rangle_{\text {obs }}^{95}(\mathrm{fb})$ \\
\hline SRinA & 38 & $27 \pm 7$ & 28.5 & $19.3_{-6.1}^{+7.0}$ & 1.41 \\
SRinB & 20 & $14.1 \pm 2.8$ & 16.3 & $10.7_{-2.6}^{+4.5}$ & 0.81 \\
SRinC & 10 & $7.1 \pm 2.9$ & 11.9 & $9.8_{-2.4}^{+3.3}$ & 0.58 \\
SRexA & 46 & $31 \pm 7$ & 32.1 & $20.3_{-3.6}^{+8.0}$ & 1.58 \\
\hline
\end{tabular}

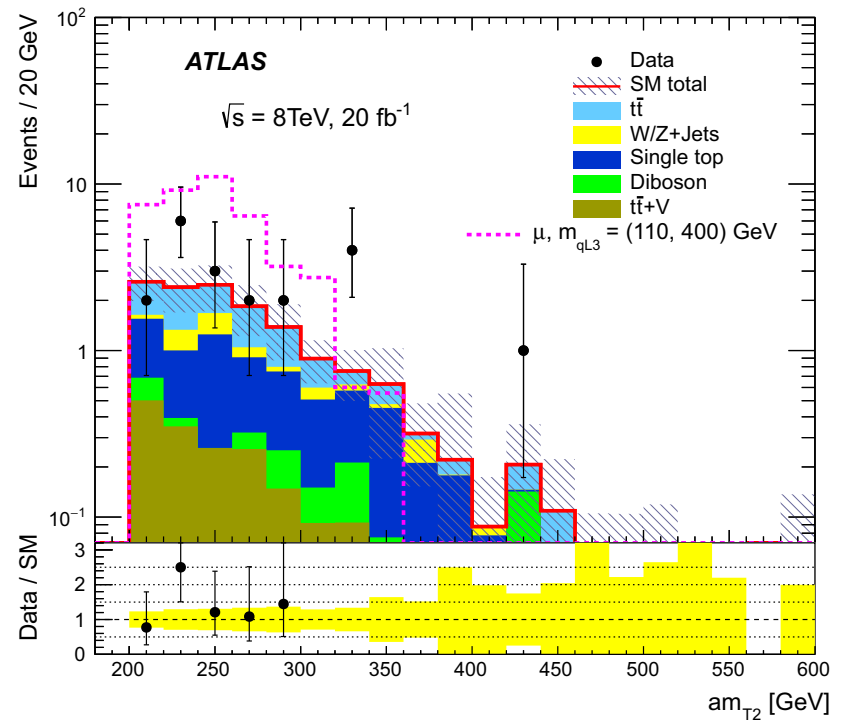

(b)

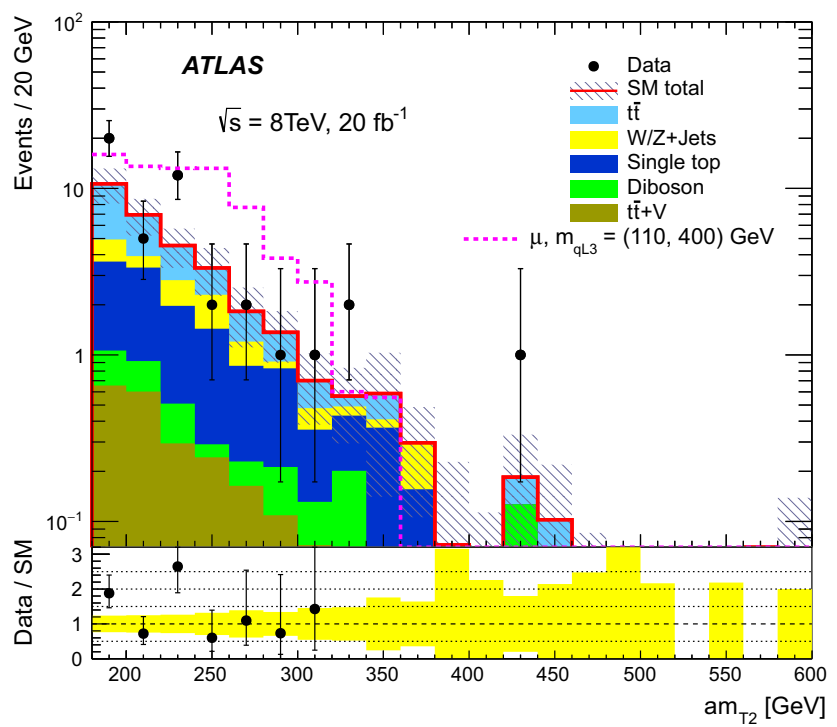

(d)

also shown. The lower panels show the ratio between the data and the SM prediction; the band includes statistical and systematic uncertainties on the SM prediction

For each combination performed, the fit setup is validated by checking that the background normalisation parameters obtained are compatible with those obtained separately by the two analyses, by verifying that no additional constraint on the nuisance parameters is introduced with respect to the individual fits, and by checking that no artificial correlation is introduced between any of the fit parameters.

The $95 \%$ CL limit derived from the combination is shown in Fig. 24, where the combined limit is compared to the individual limits obtained by the $\mathrm{t} 0 \mathrm{~L}$ and $\mathrm{t} 1 \mathrm{~L}$ analyses independently. 


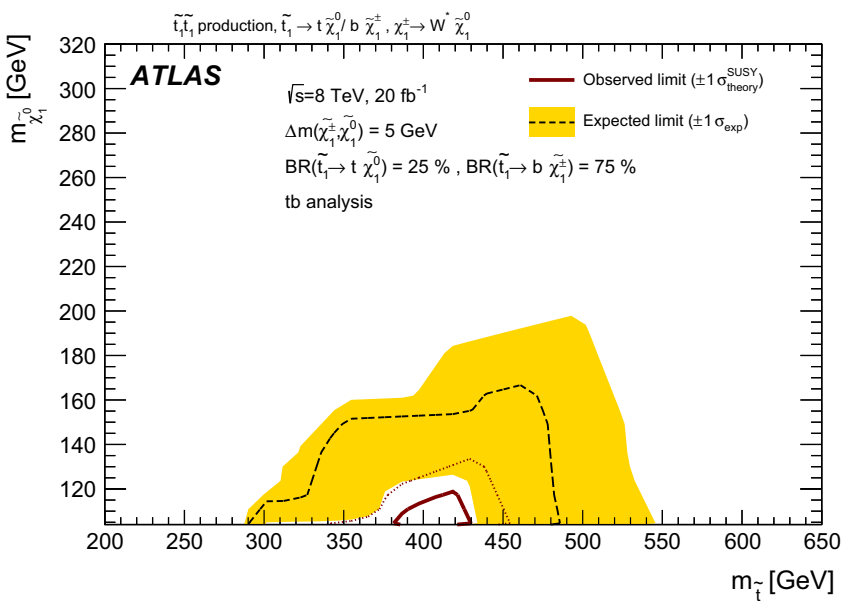

(a)

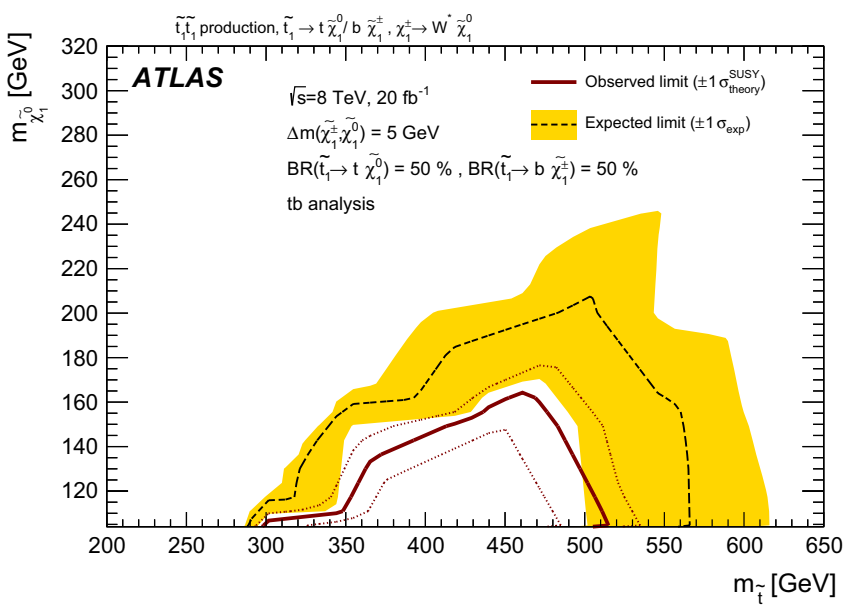

(c)

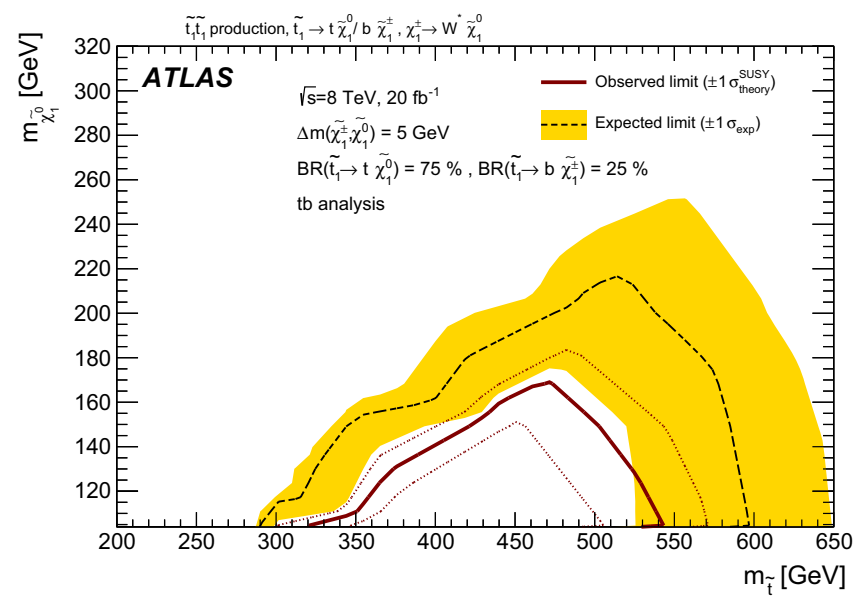

(e)

Fig. 22 Exclusion limits at $95 \% \mathrm{CL}$ from the tb signal regions for simplified models with stop decays into both $\tilde{t}_{1} \rightarrow t \tilde{\chi}_{1}^{0}$ and $\tilde{t}_{1} \rightarrow b \tilde{\chi}_{1}^{ \pm}$ and for $\operatorname{BR}\left(\tilde{t}_{1} \rightarrow t \tilde{\chi}_{1}^{0}\right)=25,50,75 \%$ (in descending rows) for the grids $\Delta m\left(\tilde{\chi}_{1}^{ \pm}, \tilde{\chi}_{1}^{0}\right)=5,20$ (left, right columns). The black dashed line indicates the expected limit, and the yellow band indicates the

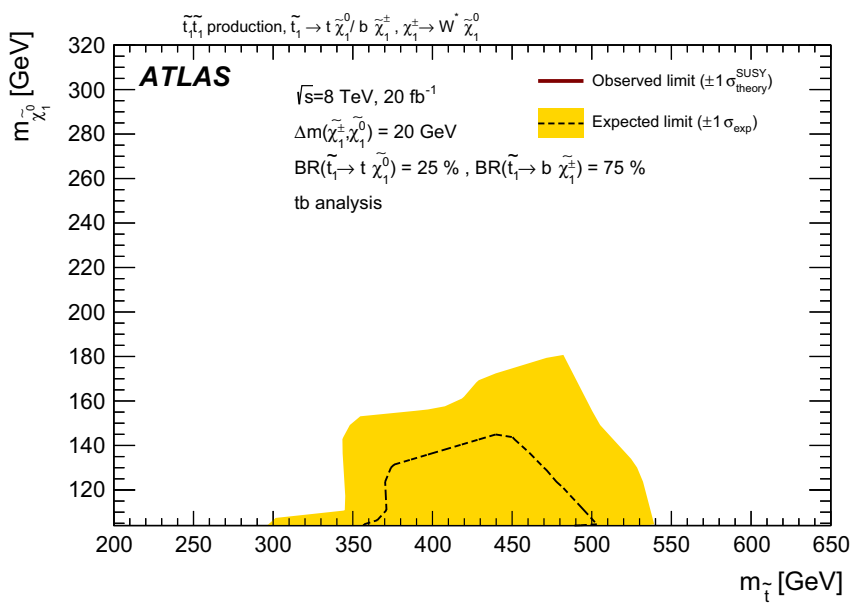

(b)

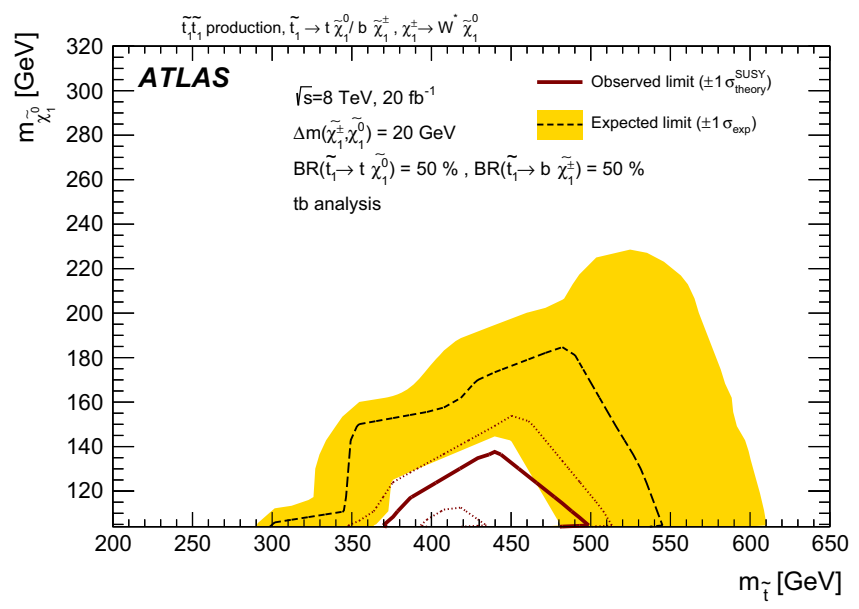

(d)

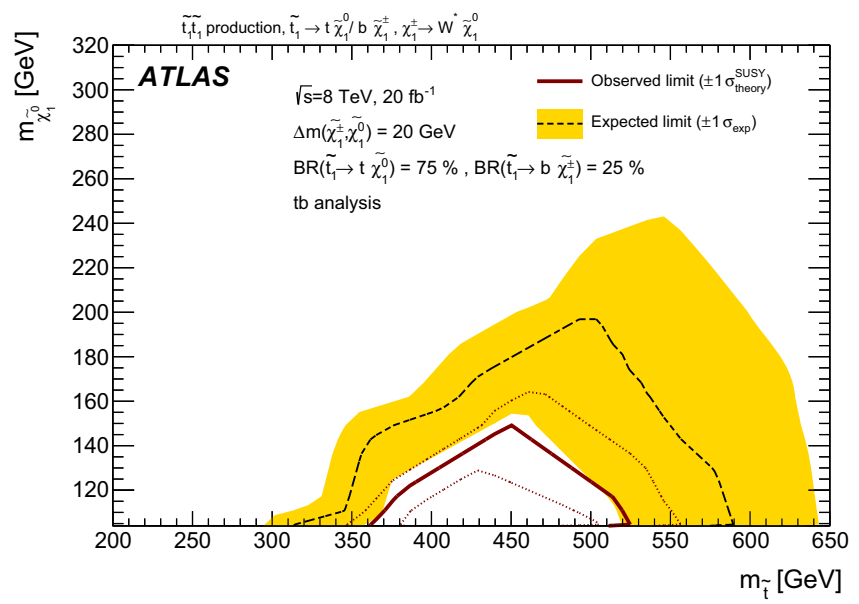

(f)

$\pm 1 \sigma$ uncertainties, which include all uncertainties except the theoretical uncertainties in the signal. The red solid line indicates the observed limit, and the red dotted lines indicate the sensitivity to $\pm 1 \sigma$ variations of the signal theoretical uncertainties. For each point the SR giving the best expected significance is used 


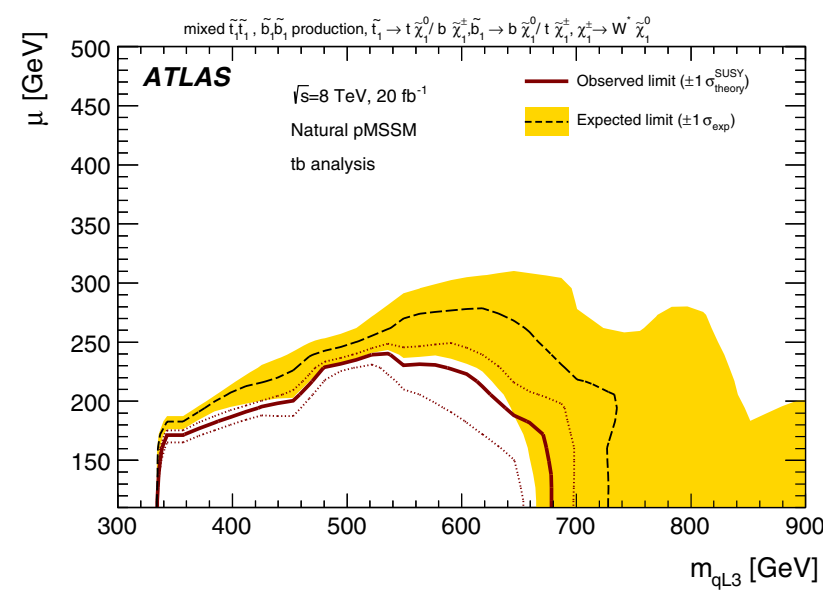

Fig. 23 Exclusion limits at $95 \% \mathrm{CL}$ from the tb signal regions for the natural pMSSM model. The black dashed line indicates the expected limit, and the yellow band indicates the $\pm 1 \sigma$ uncertainties, which include all uncertainties except the theoretical uncertainties in the signal. The red solid line indicates the observed limit, and the red dotted lines indicate the sensitivity to $\pm 1 \sigma$ variations of the signal theoretical uncertainties. For each point the SR giving the best expected significance is used

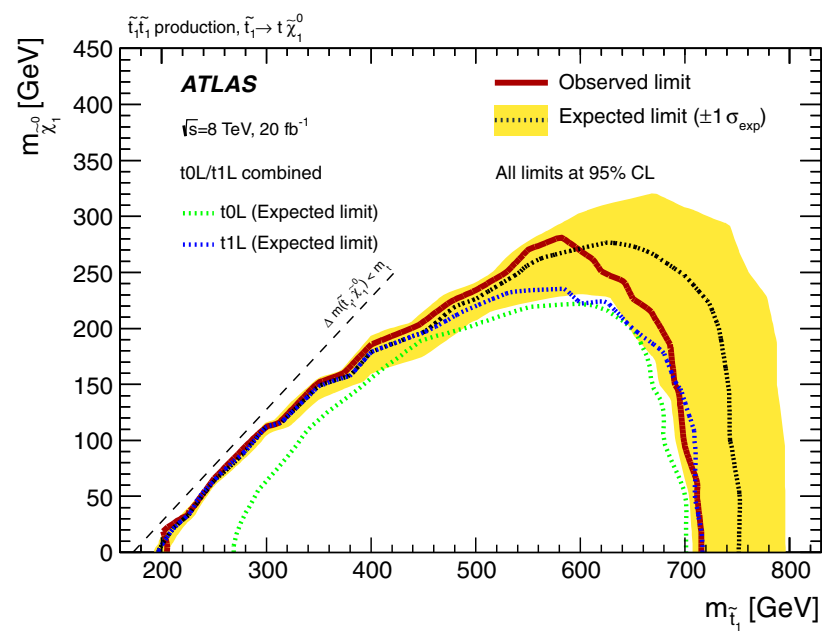

Fig. 24 Combined exclusion limits at $95 \% \mathrm{CL}$ in the scenario where both stops decay exclusively via $\tilde{t}_{1} \rightarrow t \tilde{\chi}_{1}^{0}$. The black dashed line indicates the expected limit, and the yellow band indicates the $\pm 1 \sigma$ uncertainties, which include all uncertainties except the theoretical uncertainties in the signal. The red solid line indicates the observed limit. For comparison the dotted green and blue lines show the expected limits from the standalone t0L and $11 \mathrm{~L}$ analyses

\section{Signal generation details}

Several SUSY models are considered throughout this paper. This section provides the details of how these signal models are generated. For all SUSY models discussed below, the detector response is simulated by passing the generated events through a detector simulation [125] based on GEANT4 [126] or through a fast simulation using a para- metric response to the showers in the electromagnetic and hadronic calorimeters [127] and GEANT4-based simulation elsewhere. All samples are produced with a varying number of simulated minimum-bias interactions overlaid on the hard-scattering event to account for multiple $p p$ interactions in the same or nearby bunch crossings (pileup). The simulation is reweighted to match the number of minimum bias interactions in data, which varies between approximately 10 and 30 interactions in each bunch crossing. Corrections are applied to the simulated samples to account for differences between data and simulation for the trigger and reconstruction efficiencies, momentum scale and resolution of the final-state objects, including the efficiency of identifying jets originating from the fragmentation of $b$-quarks, together with the probability for mis-tagging light-flavour and charm quarks.

Simplified models The signal samples for the scenario where both stops decay to a top quark and a neutralino are generated using Herwig++ 2.5.2 [128] interfaced to PYTHIA 6.426 [129]. The neutralino is fixed to be a pure bino, enhancing the decay of the $\tilde{t}_{\mathrm{R}}$ component of $\tilde{t}_{1}$ to a right-handed top quark. Signal samples where the two stops decay as $\tilde{t}_{1} \rightarrow b \tilde{\chi}_{1}^{ \pm}$are generated with MadGraph 5.1.4.8 [130]. For models where the $W$ boson is onshell, the $\tilde{t}_{1}$ decay is treated by MadGraph, while if the $W$ is off-shell, PYTHIA is used to decay the $\tilde{t}_{1}$. In these samples, the $\tilde{t}_{1}$ is assumed to be mostly a $\tilde{t}_{\mathrm{L}}$, and the chargino is assumed to decay through $\tilde{\chi}_{1}^{ \pm} \rightarrow W^{(*)} b$ with a branching ratio of $100 \%$. Several assumptions about the chargino masses are considered as described in the body of the paper.

Models in which the stop is assumed to decay either as $\tilde{t}_{1} \rightarrow t \tilde{\chi}_{1}^{0}$ or $\tilde{t}_{1} \rightarrow b \tilde{\chi}_{1}^{ \pm}$with different branching ratios are obtained by appropriately weighting three samples: one where both stops decay through $\tilde{t}_{1} \rightarrow t \tilde{\chi}_{1}^{0}$, a second one where both stops decay through $\tilde{t}_{1} \rightarrow b \tilde{\chi}_{1}^{ \pm}$, and a third one, where one of the two pair-produced stops decays as $\tilde{t}_{1} \rightarrow t \tilde{\chi}_{1}^{0}$ and the other one decays as $\tilde{t}_{1} \rightarrow b \tilde{\chi}_{1}^{ \pm}$. This last sample is generated with MadGraph, the $\tilde{t}_{1}$ is assumed to be a maximal mixing of $\tilde{t}_{\mathrm{L}}$ and $\tilde{t}_{\mathrm{R}}$. The mass of the chargino in this sample satisfies the gauge-universality relation $m_{\tilde{\chi}_{1}^{ \pm}}=2 m_{\tilde{\chi}_{1}^{0}}$.

The three-body stop decay samples are generated with Herwig++, which performs the matrix element calculation of the three-body decay. The four-body stop decay samples are generated with MadGraph.

For all samples considered, the mass of the bottom quark is fixed to $4.8 \mathrm{GeV}$ and its width is assumed to be zero.

The samples where both stops decay as $\tilde{t}_{1} \rightarrow c \tilde{\chi}_{1}^{0}$ are generated with MadGraph, with one additional parton from the matrix element. Similarly to the case of the limit derived as a function of the stop branching ratio into $\tilde{t}_{1} \rightarrow t \tilde{\chi}_{1}^{0}$, 
the samples with both stops decaying as $\tilde{t}_{1} \rightarrow c \tilde{\chi}_{1}^{0}$ and those where both stops decay through the four-body decay are appropriately weighted and combined with a third sample where one stop decays as $\tilde{t}_{1} \rightarrow c \tilde{\chi}_{1}^{0}$ and the other decays through the four-body decay to produce a sample of arbitrary branching ratio into $\tilde{t}_{1} \rightarrow c \tilde{\chi}_{1}^{0}$ (assuming that $\tilde{t}_{1} \rightarrow c \tilde{\chi}_{1}^{0}$ and the four-body decay are the only possible stop decays). Such mixed samples are also generated with MadGraph.

Sbottom pair production samples are also all produced with MadGraph interfaced to PYTHIA, and no more than one additional parton is added to the matrix element. The PDF set used for all signal samples is CTEQ6L1 [131].

pMSSM models In all cases, the particle spectra are generated with SOFTSUSY 3.3.3 [132], while sparticles decays are calculated with SUSY-HIT [133] (SDECAY 1.3b and HDECAY 3.4). The simulated signal events are generated using Herwig++ 2.6.3 [128] with the CTEQ6L1 PDF set.

\section{References}

1. H. Miyazawa, Prog. Theor. Phys. 36(6), 1266-1276 (1966)

2. P. Ramond, Phys. Rev. D 3, 2415-2418 (1971)

3. Y. Golfand, E. Likhtman, JETP Lett. 13, 323-326 (1971)

4. A. Neveu, J.H. Schwarz, Nucl. Phys. B 31, 86-112 (1971)

5. A. Neveu, J.H. Schwarz, Phys. Rev. D 4, 1109-1111 (1971)

6. J. Gervais, B. Sakita, Nucl. Phys. B 34, 632-639 (1971)

7. D. Volkov, V. Akulov, Phys. Lett. B 46, 109-110 (1973)

8. J. Wess, B. Zumino, Phys. Lett. B 49, 52-54 (1974)

9. J. Wess, B. Zumino, Nucl. Phys. B 70, 39-50 (1974)

10. S. Weinberg, Phys. Rev. D 13, 974-996 (1976)

11. E. Gildener, Phys. Rev. D 14, 1667-1672 (1976)

12. S. Weinberg, Phys. Rev. D 19, 1277-1280 (1979)

13. L. Susskind, Phys. Rev. D 20, 2619-2625 (1979)

14. R. Barbieri, G. Giudice, Nucl. Phys. B 306, 63-76 (1988)

15. B. de Carlos, J. Casas, Phys. Lett. B 309, 320-328 (1993). arXiv:hep-ph/9303291

16. ATLAS Collaboration, JHEP 09, 015 (2014). arXiv:1406.1122 [hep-ex]

17. ATLAS Collaboration, JHEP 1411, 118 (2014). arXiv:1407.0583 [hep-ex]

18. ATLAS Collaboration, JHEP 1406, 124 (2014). arXiv:1403.4853 [hep-ex]

19. ATLAS Collaboration, Phys. Rev. D 90(5), 052008 (2014). arXiv: 1407.0608 [hep-ex]

20. ATLAS Collaboration, Eur. Phys. J. C 74, 2883 (2014). arXiv: 1403.5222 [hep-ex]

21. ATLAS Collaboration, JHEP 1310, 189 (2013). arXiv:1308.2631 [hep-ex]

22. ATLAS Collaboration, Phys. Lett. B 715, 44-60 (2012). arXiv:1204.6736 [hep-ex]

23. ATLAS Collaboration, Phys. Rev. Lett. 109, 211802 (2012). arXiv: 1208.1447 [hep-ex]

24. ATLAS Collaboration, Eur. Phys. J. C 72, 2237 (2012). arXiv: 1208.4305 [hep-ex]

25. ATLAS Collaboration, JHEP 11, 094 (2012). arXiv:1209.4186 [hep-ex]

26. ATLAS Collaboration, Phys. Lett. B 720, 13-31 (2013). arXiv:1209.2102 [hep-ex]
27. CMS Collaboration, Phys. Rev. Lett. 111(8), 081802 (2013). arXiv:1212.6961 [hep-ex]

28. CMS Collaboration, JHEP 01, 077 (2013). arXiv:1210.8115 [hepex]

29. CMS Collaboration, Eur. Phys. J. C 73, 2568 (2013) arXiv:1303.2985 [hep-ex]

30. CMS Collaboration, Eur. Phys. J. C 73, 2677 (2013). arXiv:1308.1586 [hep-ex]

31. CMS Collaboration, Phys. Rev. Lett. 112, 161802 (2014) arXiv: 1312.3310 [hep-ex]

32. CMS Collaboration. arXiv:1503.08037 [hep-ex]

33. CMS Collaboration, Phys. Lett. B 736, 371-397 (2014) arXiv:1405.3886 [hep-ex]

34. CMS Collaboration, Phys. Lett. B 745, 5-28 (2015). arXiv:1412.4109 [hep-ex]

35. CMS Collaboration, JHEP 1303, 037 (2013). arXiv:1212.6194 [hep-ex]

36. ATLAS Collaboration, JHEP 1501, 068 (2015). arXiv:1411.6795 [hep-ex]

37. ATLAS Collaboration, Phys. Rev. D 88(11), 112003 (2013). arXiv:1310.6584 [hep-ex]

38. P. Fayet, Phys. Lett. B 64, 159-162 (1976)

39. P. Fayet, Phys. Lett. B 69, 489-494 (1977)

40. G.R. Farrar, P. Fayet, Phys. Lett. B 76, 575-579 (1978)

41. P. Fayet, Phys. Lett. B 84, 416-420 (1979)

42. S. Dimopoulos, H. Georgi, Nucl. Phys. B 193, 150-162 (1981)

43. W. Beenakker, R. Höpker, M. Spira (1996). arXiv:hep-ph/9611232

44. M. Krämer et al. arXiv:1206.2892 [hep-ph]

45. W. Beenakker et al., Nucl. Phys. B 515, 3-14 (1998)

46. W. Beenakker et al., JHEP 08, 098 (2010). arXiv:1006.4771 [hep$\mathrm{ph}]$

47. W. Beenakker et al., Int. J. Mod. Phys. A 26, 2637-2664 (2011)

48. R. Grober, M. Muhlleitner, E. Popenda, A. Wlotzka. arXiv:1502.05935 [hep-ph]

49. G. D’Ambrosio, G. Giudice, G. Isidori, A. Strumia, Nucl. Phys. B 645, 155-187 (2002). arXiv:hep-ph/0207036

50. R. Grober, M. Muhlleitner, E. Popenda, A. Wlotzka. arXiv: 1408.4662 [hep-ph]

51. A.H. Chamseddine, R. Arnowitt, P. Nath, Phys. Rev. Lett. 49, 970 (1982)

52. R. Barbieri, S. Ferrara, C.A. Savoy, Phys. Lett. B 119, 343 (1982)

53. L.E. Ibanez, Phys. Lett. B 118, 73 (1982)

54. L.J. Hall, J.D. Lykken, S. Weinberg, Phys. Rev. D 27, 2359-2378 (1983)

55. N. Ohta, Prog. Theor. Phys. 70, 542 (1983)

56. G.L. Kane, C.F. Kolda, L. Roszkowski, J.D. Wells, Phys. Rev. D 49, 6173-6210 (1994)

57. MSSM Working Group Collaboration, A. Djouadi et al. arXiv:hep-ph/9901246

58. M. Baak et al., Eur. Phys. J. C 75(4), 153 (2015). arXiv: 1410.1280 [hep-ex]

59. G. Cowan, K. Cranmer, E. Gross, O. Vitells, Eur. Phys. J. C 71, 1554 (2011). arXiv:1007.1727 [physics.data-an]

60. T. Junk, Nucl. Instrum. Methods A 434, 435-443 (1999). arXiv:hep-ex/9902006

61. A.L. Read, J. Phys. G 28, 2693-2704 (2002)

62. ATLAS Collaboration, JHEP 1410, 24 (2014). arXiv:1407.0600 [hep-ex]

63. ATLAS Collaboration, JHEP 1406, 035 (2014). arXiv:1404.2500 [hep-ex]

64. ATLAS Collaboration, Phys. Rev. Lett. 114(14), 142001 (2015). arXiv: 1412.4742 [hep-ex]

65. ATLAS Collaboration, Eur. Phys. J. C 74(10), 3109 (2014). arXiv:1406.5375 [hep-ex] 
66. LEP SUSY Working Group (Aleph, Delphi, L3, Opal), Notes lepsusywg/01-03.1 and 04-02.1. (2004). http://lepsusy.web.cern. ch/lepsusy/. Accessed 20 Oct 2015

67. ALEPH Collaboration, A. Heister et al., Phys. Lett. B 583, 247263 (2004)

68. DELPHI Collaboration, J. Abdallah et al., Eur. Phys. J. C 31, 421-479 (2003). arXiv:hep-ex/0311019

69. L3 Collaboration, M. Acciarri et al., Phys. Lett. B 472, 420-433 (2000). arXiv:hep-ex/9910007

70. OPAL Collaboration, G. Abbiendi et al., Eur. Phys. J. C 35, 1-20 (2004). arXiv:hep-ex/0401026

71. M. Ciuchini et al., JHEP 9810, 008 (1998). arXiv:hep-ph/9808328

72. ATLAS Collaboration, JHEP (2015). arXiv:1508.06608 [hep-ex]

73. ATLAS Collaboration, JHEP 1409, 176 (2014). arXiv: 1405.7875 [hep-ex]

74. ATLAS Collaboration, JHEP 1504, 116 (2015). arXiv:1501.03555 [hep-ex]

75. ATLAS Collaboration, JHEP 1310, 130 (2013). arXiv:1308.1841 [hep-ex]

76. ATLAS Collaboration, Phys. Rev. Lett. 114(16), 161801 (2015). arXiv:1501.01325 [hep-ex]

77. CMS Collaboration, JHEP 1505, 078 (2015). arXiv:1502.04358 [hep-ex]

78. CMS Collaboration, JHEP 1401, 163 (2014). arXiv:1311.6736

79. CMS Collaboration, Phys. Rev. D 91, 052018 (2015). arXiv:1502.00300 [hep-ex]

80. CMS Collaboration, JHEP 1406, 055 (2014). arXiv:1402.4770 [hep-ex]

81. CMS Collaboration, Phys. Lett. B 733, 328-353 (2014). arXiv:1311.4937 [hep-ex]

82. CMS Collaboration, Phys. Lett. B 725, 243-270 (2013). arXiv: 1305.2390 [hep-ex]

83. ATLAS Collaboration, JHEP 1411, 056 (2014). arXiv:1409.6064 [hep-ex]

84. CMS Collaboration, JHEP 1410, 160 (2014), arXiv:1408.3316 [hep-ex]

85. A. Delgado et al., Eur. Phys. J. C 73(3), 2370 (2013). arXiv: 1212.6847 [hep-ph]

86. M. Papucci, J.T. Ruderman, A. Weiler, JHEP 1209, 035 (2012). arXiv: 1110.6926 [hep-ph]

87. M.W. Cahill-Rowley, J.L. Hewett, A. Ismail, T.G. Rizzo, Phys. Rev. D 86, 075015 (2012). arXiv: 1206.5800 [hep-ph]

88. N. Arkani-Hamed, A. Delgado, G. Giudice, Nucl. Phys. B 741, 108-130 (2006). arXiv:hep-ph/0601041

89. (2015). https://atlas.web.cern.ch/Atlas/GROUPS/PHYSICS/ PAPERS/SUSY-2014-07/hepdata_info.pdf. Accessed 20 Oct 2015

90. The Durham HepData Project (2015). http://hepdata.cedar.ac.uk/ view/ins1380183. Accessed 20 Oct 2015

91. ATLAS Collaboration, JINST 3, S08003 (2008)

92. ATLAS Collaboration, Eur. Phys. J. C 73, 2518 (2013). arXiv: 1302.4393 [hep-ex]

93. ATLAS Collaboration, ATLAS-CONF-2010-069 (2010). http:// cdsweb.cern.ch/record/1281344. Accessed 20 Oct 2015

94. W. Lampl et al., ATL-LARG-PUB-2008-002 (2008). http:// cdsweb.cern.ch/record/1099735. Accessed 20 Oct 2015

95. M. Cacciari, G.P. Salam, G. Soyez, JHEP 04, 063 (2008). arXiv:0802.1189 [hep-ph]

96. M. Cacciari, G.P. Salam, Phys. Lett. B 641, 57-61 (2006). arXiv:hep-ph/0512210

97. M. Cacciari, G.P. Salam, G. Soyez, Eur. Phys. J. C 72, 1896 (2012). arXiv:1111.6097 [hep-ph]

98. C. Issever, K. Borras, D. Wegener, Nucl. Instrum. Methods A 545, 803-812 (2005). arXiv:physics/0408129

99. M. Cacciari, G.P. Salam, Phys. Lett. B 659, 119-126 (2008). arXiv:0707.1378 [hep-ph]
100. ATLAS Collaboration, Eur. Phys. J. C 73, 2304 (2013). arXiv:1112.6426 [hep-ex]

101. ATLAS Collaboration, ATLAS-CONF-2012-043 (2012). http:// cdsweb.cern.ch/record/1435197. Accessed 20 Oct 2015

102. ATLAS Collaboration, ATLAS-CONF-2011-089 (2011). http:// cdsweb.cern.ch/record/1356198. Accessed 20 Oct 2015

103. ATLAS Collaboration, ATLAS-CONF-2011-102 (2011). http:// cdsweb.cern.ch/record/1369219. Accessed 20 Oct 2015

104. ATLAS Collaboration, Eur. Phys. J. C 72, 1909 (2012) arXiv: 1110.3174 [hep-ex]

105. ATLAS Collaboration, ATLAS-CONF-2011-021 (2011). http:// cdsweb.cern.ch/record/1336750. Accessed 20 Oct 2015

106. ATLAS Collaboration, ATLAS-CONF-2011-063 (2011). http:// cdsweb.cern.ch/record/1345743. Accessed 20 Oct 2015

107. C. Lester, D. Summers, Phys. Lett. B 463, 99-103 (1999). arXiv:hep-ph/9906349

108. A. Barr, C. Lester, P. Stephens, J. Phys. G 29, 2343-2363 (2003). arXiv:hep-ph/0304226

109. M.L. Graesser, J. Shelton, Phys. Rev. Lett. 111(12), 121802 (2013). arXiv:1212.4495 [hep-ph]

110. D.R. Tovey, JHEP 0804, 034 (2008). arXiv:0802.2879 [hep-ph]

111. T. Eifert, B. Nachman, Phys. Lett. B 743, 218-223 (2015). arXiv: 1410.7025 [hep-ph]

112. ATLAS Collaboration, Eur. Phys. J. C 75(7), 330 (2015) arXiv:1503.05427 [hep-ex]

113. ATLAS Collaboration, Phys. Lett. B 712, 289-308 (2012). arXiv:1203.6232 [hep-ex]

114. CMS Collaboration, Eur. Phys. J. C 73(10), 2610 (2013). arXiv:1306.1126 [hep-ex]

115. CMS Collaboration, Phys. Lett. B 721, 190-211 (2013). arXiv: 1301.4698 [hep-ex]

116. K. Rolbiecki, K. Sakurai, JHEP 1309, 004 (2013). arXiv: 1303.5696 [hep-ph]

117. J.S. Kim, K. Rolbiecki, K. Sakurai, J. Tattersall, JHEP 1412, 010 (2014). arXiv:1406.0858 [hep-ph]

118. D. Curtin, P. Meade, P.-J. Tien, Phys. Rev. D 90(11), 115012 (2014). arXiv:1406.0848 [hep-ph]

119. T. Melia, JHEP 1201, 143 (2012). arXiv:1110.6185 [hep-ph]

120. ATLAS Collaboration, Eur. Phys. J. C 75(7), 349 (2015). arXiv:1503.05066 [hep-ex]

121. F. Cascioli et al., Phys. Lett. B 734, 210-214 (2014). arXiv:1309.5912 [hep-ph]

122. T. Gleisberg et al., JHEP 02, 007 (2009). arXiv:0811.4622 [hep$\mathrm{ph}]$

123. F. Cascioli, P. Maierhofer, S. Pozzorini, Phys. Rev. Lett. 108, 111601 (2012). arXiv:1111.5206 [hep-ph]

124. ATLAS Collaboration, Phys. Rev. D 90(7), 072004 (2014). arXiv: 1407.0371 [hep-ex]

125. ATLAS Collaboration, Eur. Phys. J. C 70, 823-874 (2010). arXiv: 1005.4568 [physics.ins-det]

126. GEANT4 Collaboration, S. Agostinelli et al., Nucl. Instrum. Methods A 506, 250-303 (2003)

127. ATLAS Collaboration, ATL-PHYS-PUB-2010-013 (2010). http://cdsweb.cern.ch/record/1300517. Accessed 20 Oct 2015

128. M. Bähr et al., Eur. Phys. J. C 58, 639-707 (2008). arXiv:0803.0883 [hep-ph]

129. T. Sjöstrand, S. Mrenna, P.Z. Skands, JHEP 05, 026 (2006). arXiv:hep-ph/0603175

130. J. Alwall et al., JHEP 06, 128 (2011). arXiv:1106.0522 [hep-ph]

131. J. Pumplin et al., JHEP 07, 012 (2002). arXiv:hep-ph/0201195

132. B. Allanach, Comput. Phys. Commun. 143, 305-331 (2002). arXiv:hep-ph/0104145

133. A. Djouadi, M.M. Muhlleitner, M. Spira, Acta Phys. Polon. B 38, 635-644 (2007). arXiv:hep-ph/0609292 


\section{ATLAS Collaboration}

G. Aad ${ }^{85}$, B. Abbott ${ }^{113}$, J. Abdallah ${ }^{151}$, O. Abdinov ${ }^{11}$, R. Aben ${ }^{107}$, M. Abolins ${ }^{90}$, O. S. AbouZeid ${ }^{158}$, H. Abramowicz ${ }^{153}$, H. Abreu ${ }^{152}$, R. Abreu ${ }^{116}$, Y. Abulaiti ${ }^{146 a, 146 b}$, B. S. Acharya ${ }^{164 a, 164 b, a}$, L. Adamczyk ${ }^{38 a}$, D. L. Adams ${ }^{25}$, J. Adelman ${ }^{108}$, S. Adomeit ${ }^{100}$, T. Adye ${ }^{131}$, A. A. Affolder ${ }^{74}$, T. Agatonovic-Jovin ${ }^{13}$, J. Agricola ${ }^{54}$, J. A. Aguilar-Saavedra ${ }^{126 a, 126 f}$,

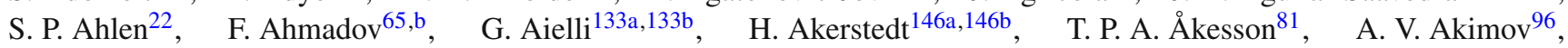
G. L. Alberghi ${ }^{20 a, 20 b}$, J. Albert ${ }^{169}$, S. Albrand ${ }^{55}$, M. J. Alconada Verzini ${ }^{71}$, M. Aleksa ${ }^{30}$, I. N. Aleksandrov ${ }^{65}$,

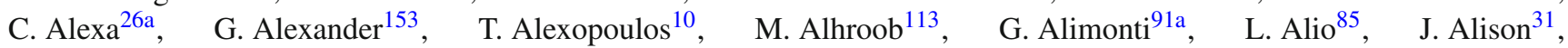
S. P. Alkire ${ }^{35}$, B. M. M. Allbrooke ${ }^{149}$, P. P. Allport ${ }^{74}$, A. Aloisio ${ }^{104 a, 104 b}$, A. Alonso ${ }^{36}$, F. Alonso ${ }^{71}$, C. Alpigiani ${ }^{76}$, A. Altheimer ${ }^{35}$, B. Alvarez Gonzalez ${ }^{30}$, D. Álvarez Piqueras ${ }^{167}$, M. G. Alviggi 104a,104b, B. T. Amadio ${ }^{15}$, K. Amako ${ }^{66}$, Y. Amaral Coutinho ${ }^{24 a}$, C. Amelung ${ }^{23}$, D. Amidei ${ }^{89}$, S. P. Amor Dos Santos ${ }^{126 a, 126 c}$, A. Amorim ${ }^{126 a, 126 b}$, S. Amoroso ${ }^{48}$,

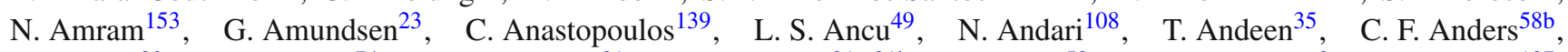
G. Anders $^{30}$, J. K. Anders $^{74}$, K. J. Anderson ${ }^{31}$, A. Andreazza ${ }^{91 a, 91 b}$, V. Andrei ${ }^{58 a}$, S. Angelidakis ${ }^{9}$, I. Angelozzi ${ }^{107}$, P. Anger ${ }^{44}$, A. Angerami ${ }^{35}$, F. Anghinolfi ${ }^{30}$, A. V. Anisenkov ${ }^{109, c}$, N. Anjos ${ }^{12}$, A. Annovi ${ }^{124 a, 124 b}$, M. Antonellii ${ }^{47}$, A. Antonov ${ }^{98}$, J. Antos ${ }^{144 b}$, F. Anulli ${ }^{132 a}$, M. Aoki ${ }^{66}$, L. Aperio Bella ${ }^{18}$, G. Arabidze ${ }^{90}$, Y. Arai ${ }^{66}$, J. P. Araque ${ }^{126 a}$, A. T. H. Arce ${ }^{45}$, F. A. Arduh ${ }^{71}$, J-F. Arguin ${ }^{95}$, S. Argyropoulos ${ }^{42}$, M. Arik ${ }^{19 a}$, A. J. Armbruster ${ }^{30}$, O. Arnaez $^{30}$, V. Arnal ${ }^{82}$, H. Arnold ${ }^{48}$, M. Arratia ${ }^{28}$, O. Arslan ${ }^{21}$, A. Artamonov ${ }^{97}$, G. Artoni ${ }^{23}$, S. Asai ${ }^{155}$, N. Asbah ${ }^{42}$, A. Ashkenazi ${ }^{153}$, B. Åsman 146a,146b, L. Asquith ${ }^{149}$, K. Assamagan ${ }^{25}$, R. Astalos ${ }^{144 a}$, M. Atkinson ${ }^{165}$, N. B. Atlay ${ }^{141}$, B. Auerbach ${ }^{6}$,

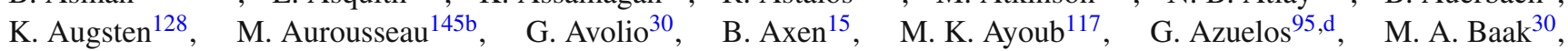

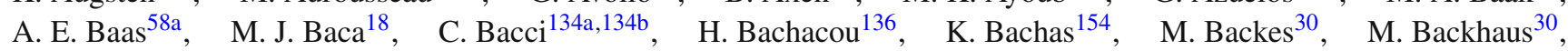
P. Bagiacchi132a,132b , P. Bagnaia132a,132b, Y. Bai ${ }^{33 a}$, T. Bain ${ }^{35}$, J. T. Baines ${ }^{131}$, O. K. Baker ${ }^{176}$, E. M. Baldin ${ }^{109, c}$, P. Balek ${ }^{129}$, T. Balestri ${ }^{148}$, F. Balli ${ }^{84}$, E. Banas ${ }^{39}$, Sw. Banerjee ${ }^{173}$, A. A. E. Bannoura ${ }^{175}$, H. S. Bansil ${ }^{18}$, L. Barak ${ }^{30}$, E. L. Barberio $^{88}$, D. Barberis ${ }^{50,50 b}$, M. Barbero ${ }^{85}$, T. Barillari ${ }^{101}$, M. Barisonzi ${ }^{164 a, 164 b}$, T. Barklow ${ }^{143}$, N. Barlow ${ }^{28}$, S. L. Barnes ${ }^{84}$, B. M. Barnett ${ }^{131}$ ， R. M. Barnett ${ }^{15}$, Z. Barnovska ${ }^{5}$, A. Baroncelli134a, G. Barone ${ }^{23}$, A. J. Barr ${ }^{120}$, F. Barreiro ${ }^{82}$, J. Barreiro Guimarães da Costa $^{57}, \quad$ R. Bartoldus ${ }^{143}, \quad$ A. E. Barton ${ }^{72}, \quad$ P. Bartos ${ }^{144 a}$, A. Basalaev ${ }^{123}$,

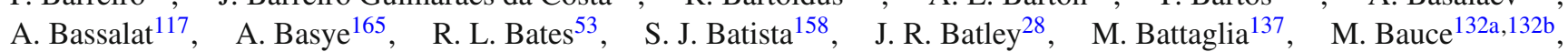
F. Bauer ${ }^{136}$, H. S. Bawa ${ }^{143, \text { e, }}$, J. B. Beacham ${ }^{111}$, M. D. Beattie ${ }^{72}$, T. Beau ${ }^{80}$, P. H. Beauchemin ${ }^{161}$, R. Beccherle ${ }^{124 a, 124 b}$, P. Bechtle ${ }^{21}$, H. P. Beck ${ }^{17, f}$, K. Becker ${ }^{120}$, M. Becker ${ }^{83}$, S. Becker ${ }^{100}$, M. Beckingham ${ }^{170}$, C. Becot ${ }^{117}$, A. J. Beddall ${ }^{19 b}$, A. Beddall ${ }^{19 b}$, V. A. Bednyakov ${ }^{65}$, C. P. Bee ${ }^{148}$, L. J. Beemster ${ }^{107}$, T. A. Beermann ${ }^{175}$, M. Begel ${ }^{25}$, J. K. Behr ${ }^{120}$, C. Belanger-Champagne ${ }^{87}$, W. H. Bell ${ }^{49}$, G. Bella ${ }^{153}$, L. Bellagamba ${ }^{20 a}$, A. Bellerive ${ }^{29}$, M. Bellomo ${ }^{86}$, K. Belotskiy ${ }^{98}$, O. Beltramello ${ }^{30}$, O. Benary ${ }^{153}$, D. Benchekroun ${ }^{135 a}$, M. Bender ${ }^{100}$, K. Bendtz ${ }^{146,146 b}$, N. Benekos ${ }^{10}$, Y. Benhammou ${ }^{153}$, E. Benhar Noccioli ${ }^{49}$, J. A. Benitez Garcia ${ }^{159 b}$, D. P. Benjamin ${ }^{45}$, J. R. Bensinger ${ }^{23}$, S. Bentvelsen ${ }^{107}$, L. Beresford ${ }^{120}$, M. Beretta ${ }^{47}$, D. Berge ${ }^{107}$, E. Bergeaas Kuutmann ${ }^{166}, \quad$ N. Berger ${ }^{5}$, F. Berghaus ${ }^{169}$, J. Beringer ${ }^{15}$, C. Bernard ${ }^{22}$, N. R. Bernard ${ }^{86}$, C. Bernius ${ }^{110}$, F. U. Bernlochner ${ }^{21}$, T. Berry ${ }^{77}$, P. Berta ${ }^{129}$, C. Bertella ${ }^{83}$, G. Bertoli ${ }^{146 a, 146 b}$, F. Bertolucci124a,124b, C. Bertsche ${ }^{113}$, D. Bertsche ${ }^{113}$, M. I. Besana ${ }^{91 a}$, G. J. Besjes ${ }^{36}$, O. Bessidskaia Bylund ${ }^{146 a, 146 b}$, M. Bessner ${ }^{42}$, N. Besson ${ }^{136}$, C. Betancourt ${ }^{48}$, S. Bethke ${ }^{101}$, A. J. Bevan ${ }^{76}$, W. Bhimji ${ }^{15}$, R. M. Bianchi ${ }^{125}$, L. Bianchini ${ }^{23}$, M. Bianco ${ }^{30}$, O. Biebel ${ }^{100}$, D. Biedermann ${ }^{16}$, S. P. Bieniek ${ }^{78}$, M. Biglietti ${ }^{134 a}$, J. Bilbao De Mendizabal ${ }^{49}$, H. Bilokon ${ }^{47}$, M. Bindi ${ }^{54}$, S. Binet ${ }^{117}$, A. Bingul ${ }^{19 b}$, C. Bini ${ }^{132 a, 132 b}$, S. Biondi ${ }^{20 a, 20 b}$, C. W. Black ${ }^{150}$, J. E. Black ${ }^{143}$, K. M. Black ${ }^{22}$, D. Blackburn ${ }^{138}$, R. E. Blair ${ }^{6}$, J.-B. Blanchard ${ }^{136}$, J. E. Blanco ${ }^{77}$, T. Blazek ${ }^{144 a}$, I. Bloch ${ }^{42}$, C. Blocker ${ }^{23}$, W. Blum ${ }^{83, *}$, U. Blumenschein $^{54}$, G. J. Bobbink ${ }^{107}$, V. S. Bobrovnikov ${ }^{109, c}$, S. S. Bocchetta ${ }^{81}$, A. Bocci ${ }^{45}$, C. Bock ${ }^{100}$, M. Boehler ${ }^{48}$, $\begin{array}{ll}\text { J. A. Bogaerts } & 30\end{array}$ D. Bogavac ${ }^{13}$, A. G. Bogdanchikov ${ }^{109}$, C. Bohm ${ }^{146 a}$, V. Boisvert ${ }^{77}$, T. Bold ${ }^{38 a}$, V. Boldea ${ }^{26 a}$, A. S. Boldyrev ${ }^{99}$, M. Bomben ${ }^{80}$, M. Bona ${ }^{76}$, M. Boonekamp ${ }^{136}$, A. Borisov ${ }^{130}$, G. Borissov ${ }^{72}$, S. Borroni ${ }^{42}$, J. Bortfeldt ${ }^{100}$,

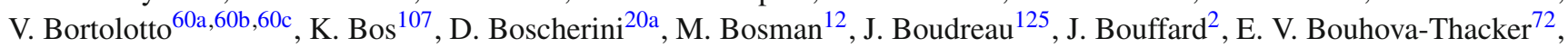
D. Boumediene ${ }^{34}$, C. Bourdarios ${ }^{117}$, N. Bousson ${ }^{114}$, A. Boveia ${ }^{30}$, J. Boyd ${ }^{30}$, I. R. Boyko ${ }^{65}$, I. Bozic ${ }^{13}$, J. Bracinik ${ }^{18}$, A. Brandt ${ }^{8}$, G. Brandt ${ }^{54}$, O. Brandt ${ }^{58 a}$, U. Bratzler ${ }^{156}$, B. Brau ${ }^{86}$, J. E. Brau ${ }^{116}$, H. M. Braun ${ }^{175, *}$, S. F. Brazzale ${ }^{164 a, 164 c}$, W. D. Breaden Madden ${ }^{53}$, K. Brendlinger ${ }^{122}$, A. J. Brennan ${ }^{88}$, L. Brenner ${ }^{107}$, R. Brenner ${ }^{166}$, S. Bressler ${ }^{172}$, K. Bristow ${ }^{145 c}$, T. M. Bristow ${ }^{46}$, D. Britton ${ }^{53}$, D. Britzger ${ }^{42}$, F. M. Brochu ${ }^{28}$, I. Brock ${ }^{21}$, R. Brock ${ }^{90}$, J. Bronner ${ }^{101}$, G. Brooijmans ${ }^{35}$, T. Brooks ${ }^{77}$, W. K. Brooks ${ }^{32 b}$, J. Brosamer ${ }^{15}$, E. Brost ${ }^{116}$, J. Brown ${ }^{55}$, P. A. Bruckman de Renstrom ${ }^{39}$, D. Bruncko ${ }^{144 b}$, R. Bruneliere ${ }^{48}$, A. Bruni ${ }^{20 a}$, G. Bruni ${ }^{20 a}$, M. Bruschi ${ }^{20 a}$, N. Bruscino ${ }^{21}$, L. Bryngemark ${ }^{81}$, T. Buanes ${ }^{14}$, Q. Buat ${ }^{142}$, P. Buchholz
141, A. G. Buckley O. Bulekov ${ }^{98}$, D. Bullock ${ }^{8}$, H. Burckhart ${ }^{30}$, S. Burdin ${ }^{74}$, B. Burghgrave ${ }^{108}$, S. Burke ${ }^{131}$, I. Burmeister ${ }^{43}$, E. Busato ${ }^{34}$, D. Büscher ${ }^{48}$, V. Büscher ${ }^{83}$, P. Bussey ${ }^{53}$, J. M. Butler ${ }^{22}$, A. I. Butt ${ }^{3}$, C. M. Buttar ${ }^{53}$, J. M. Butterworth ${ }^{78}$, P. Butti ${ }^{107}$, W. Buttinger ${ }^{25}$, A. Buzatu ${ }^{53}$, A. R. Buzykaev ${ }^{109, c}$, S. Cabrera Urbán ${ }^{167}$, D. Caforio ${ }^{128}$, V. M. Cairo ${ }^{37 a, 37 b}$, O. Cakir ${ }^{4 a}$, 
N. Calace ${ }^{49}$, P. Calafiura ${ }^{15}$, A. Calandri ${ }^{136}$, G. Calderini ${ }^{80}$, P. Calfayan ${ }^{100}$, L. P. Caloba ${ }^{24 a}$, D. Calvet ${ }^{34}$, S. Calvet ${ }^{34}$, R. Camacho Toro ${ }^{31}$, S. Camarda ${ }^{42}$, P. Camarri ${ }^{133 a, 133 b}$, D. Cameron ${ }^{119}$ ， R. Caminal Armadans ${ }^{165}$, S. Campana ${ }^{30}$, M. Campanelli ${ }^{78}$, A. Campoverde ${ }^{148}$, V. Canale ${ }^{104 a, 104 b}$, A. Canepa ${ }^{159 a}$, M. Cano Bret ${ }^{33 e}$, J. Cantero ${ }^{82}$, R. Cantrill ${ }^{126 a}$, T. Cao ${ }^{40}$, M. D. M. Capeans Garrido ${ }^{30}$, I. Caprini ${ }^{26 a}$, M. Caprini ${ }^{26 a}$, M. Capua ${ }^{37 a, 37 b}$, R. Caputo ${ }^{83}$, R. Cardarelli ${ }^{133 a}$, F. Cardillo ${ }^{48}$, T. Carli ${ }^{30}$, G. Carlino ${ }^{104 a}$, L. Carminati ${ }^{91 a}, 91 b$, S. Caron ${ }^{106}$, E. Carquin ${ }^{32 a}$, G. D. Carrillo-Montoya ${ }^{8}$,

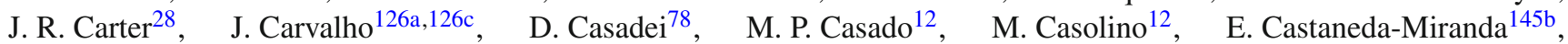
A. Castelli ${ }^{107}$, V. Castillo Gimenez ${ }^{167}$, N. F. Castro ${ }^{126 a, g}$, P. Catastini ${ }^{57}$, A. Catinaccio ${ }^{30}$, J. R. Catmore ${ }^{119}$, A. Cattai ${ }^{30}$, J. Caudron ${ }^{83}$, V. Cavaliere ${ }^{165}$, D. Cavalli ${ }^{91 a}$, M. Cavalli-Sforza ${ }^{12}$, V. Cavasinni124a,124b ${ }^{12}$ F. Ceradini ${ }^{134 a, 134 b}$, B. C. Cerio ${ }^{45}$, K. Cerny ${ }^{129}$, A. S. Cerqueira ${ }^{24 b}$, A. Cerri ${ }^{149}$, L. Cerrito ${ }^{76}$, F. Cerutti ${ }^{15}$, M. Cerv ${ }^{30}$, A. Cervelli ${ }^{17}$, S. A. Cetin ${ }^{19 c}$, A. Chafaq ${ }^{135 a}$, D. Chakraborty ${ }^{108}$, I. Chalupkova ${ }^{129}$, P. Chang ${ }^{165}$, J. D. Chapman ${ }^{28}$, D. G. Charlton ${ }^{18}$, C. C. Chau ${ }^{158}$, C. A. Chavez Barajas ${ }^{149}$, S. Cheatham ${ }^{152}$, A. Chegwidden ${ }^{90}$, S. Chekanov ${ }^{6}$, S. V. Chekulaev ${ }^{159 a}$, G. A. Chelkov ${ }^{65, h}$, M. A. Chelstowska ${ }^{89}$, C. $\mathrm{Chen}^{64}$, H. Chen ${ }^{25}$, K. Chen ${ }^{148}$, L. Chen ${ }^{33 d, i}$, S. Chen ${ }^{33 c}$, X. Chen ${ }^{33 f}$, Y. Chen ${ }^{67}$, H. C. Cheng ${ }^{89}$, Y. Cheng ${ }^{31}$, A. Cheplakov ${ }^{65}$, E. Cheremushkina ${ }^{130}$, R. Cherkaoui El Moursli ${ }^{135 e}$, V. Chernyatin ${ }^{25, *}$ E. Cheu ${ }^{7}$, L. Chevalier ${ }^{136}$, V. Chiarella ${ }^{47}$, G. Chiarelli1 ${ }^{124 a, 124 b}$, J. T. Childers ${ }^{6}$, G. Chiodini ${ }^{73 a}$, A. S. Chisholm ${ }^{18}$, R. T. Chislett ${ }^{78}$, A. Chitan ${ }^{26 a}$, M. V. Chizhov ${ }^{65}$, K. Choi $^{61}$, S. Chouridou ${ }^{9}$, B. K. B. Chow ${ }^{100}$, V. Christodoulou ${ }^{78}$, D. Chromek-Burckhart ${ }^{30}$, J. Chudoba ${ }^{127}$, A. J. Chuinard ${ }^{87}$, J. J. Chwastowski ${ }^{39}$, L. Chytka ${ }^{115}$, G. Ciapetti ${ }^{132 a, 132 b}$, A. K. Ciftci ${ }^{4 a}$, D. Cinca ${ }^{53}$, V. Cindro ${ }^{75}$, I. A. Cioara ${ }^{21}$, A. Ciocio ${ }^{15}$, Z. H. Citron ${ }^{172}$, M. Ciubancan ${ }^{26 a}$, A. Clark ${ }^{49}$, B. L. Clark ${ }^{57}$, P. J. Clark ${ }^{46}$, R. N. Clarke ${ }^{15}$, W. Cleland ${ }^{125}$, C. Clement ${ }^{146 a}$,146b, Y. Coadou ${ }^{85}$, M. Cobal ${ }^{164 a, 164 c}$, A. Coccaro ${ }^{138}$, J. Cochran ${ }^{64}$, L. Coffey $^{23}$, J. G. Cogan $^{143}$, L. Colasurdo ${ }^{106}$, B. Cole $^{35}$, S. Cole ${ }^{108}$, A. P. Colijn ${ }^{107}$, J. Collot ${ }^{55}$, T. Colombo ${ }^{58 c}$, G. Compostella $^{101}$, P. Conde Muiño ${ }^{126 a, 126 b}$, E. Coniavitis ${ }^{48}$, S. H. Connell1 ${ }^{145 b}$, I. A. Connelly ${ }^{77}$, S. M. Consonni ${ }^{11 a, 91 b}$, V. Consorti ${ }^{48}$, S. Constantinescu ${ }^{26 a}$, C. Conta ${ }^{121 a, 121 b}$, G. Conti ${ }^{30}$, F. Conventi ${ }^{104 a, j}$, M. Cooke ${ }^{15}$, B. D. Cooper ${ }^{78}$, A. M. Cooper-Sarkar ${ }^{120}$, T. Cornelissen ${ }^{175}$, M. Corradi ${ }^{20 a}$, F. Corriveau ${ }^{87, k}$, A. Corso-Radu ${ }^{163}$, A. Cortes-Gonzalez ${ }^{12}$, G. Cortiana ${ }^{101}$, G. Costa ${ }^{91 a}$, M. J. Costa ${ }^{167}$, D. Costanzo ${ }^{139}$, D. Côté ${ }^{8}$ G. Cottin ${ }^{28}$, G. Cowan ${ }^{77}$, B. E. Cox ${ }^{84}$, K. Cranmer ${ }^{110}$, G. Cree $^{29}$, S. Crépé-Renaudin ${ }^{55}, \quad$ F. Crescioli ${ }^{80}$, W. A. Cribbs ${ }^{146 a, 146 b}$, M. Crispin Ortuzar ${ }^{120}$,

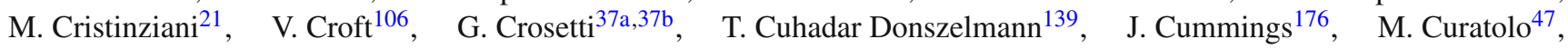
C. Cuthbert ${ }^{150}$, H. Czirr ${ }^{141}$, P. Czodrowski ${ }^{3}$, S. D’Auria ${ }^{53}$, M. D’Onofrio ${ }^{74}$, M. J. Da Cunha Sargedas De Sousa ${ }^{126 a, 126 b}$, C. $\mathrm{Da} \mathrm{Via}^{84}$, W. Dabrowski ${ }^{38 a}$, A. Dafinca ${ }^{120}$, T. Dai $^{89}$, O. Dale ${ }^{14}$, F. Dallaire ${ }^{95}$, C. Dallapiccola ${ }^{86}$, M. Dam ${ }^{36}$, J. R. Dandoy ${ }^{31}$ ， N. P. Dang ${ }^{48}$, A. C. Daniells ${ }^{18}$, M. Danninger ${ }^{168}$, M. Dano Hoffmann ${ }^{136}$, V. Dao ${ }^{48}$, G. Darbo ${ }^{50 a}$, S. Darmora ${ }^{8}$, J. Dassoulas ${ }^{3}$, A. Dattagupta ${ }^{61}$, W. Davey ${ }^{21}$, C. David ${ }^{169}$, T. Davidek ${ }^{129}$, E. Davies ${ }^{120,1}$, M. Davies ${ }^{153}$, P. Davison $^{78}$, Y. Davygora ${ }^{58 a}$, E. Dawe ${ }^{88}$, I. Dawson ${ }^{139}$, R. K. Daya-Ishmukhametova ${ }^{86}$, K. De ${ }^{8}$, R. de Asmundis ${ }^{104 a}$, A. De Benedetti ${ }^{113}$, S. De Castro ${ }^{20 a, 20 b}$, S. De Cecco ${ }^{80}$, N. De Groot ${ }^{106}$, P. de Jong ${ }^{107}$, H. De la Torre ${ }^{82}$, F. De Lorenzi ${ }^{64}$, L. De Nooij ${ }^{107}$, D. De Pedis ${ }^{132 a}$ ， A. De Salvo ${ }^{132 a}$, U. De Sanctis ${ }^{149}, \quad$ A. De Santo ${ }^{149}$ ， J. B. De Vivie De Regie ${ }^{117}$,

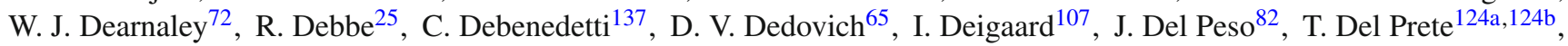
D. Delgove ${ }^{117}$, F. Deliot ${ }^{136}$, C. M. Delitzsch ${ }^{49}$, M. Deliyergiyev ${ }^{75}$, A. Dell'Acqua ${ }^{30}$, L. Dell'Asta ${ }^{22}$, M. Dell'Orso ${ }^{124 a, 124 b}$,

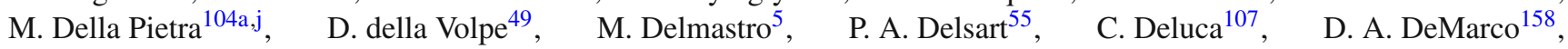
S. Demers ${ }^{176}$, M. Demichev ${ }^{65}$, A. Demilly ${ }^{80}$, S. P. Denisov ${ }^{130}$, D. Derendarz ${ }^{39}$, J. E. Derkaoui ${ }^{135 d}$, F. Derue ${ }^{80}$, P. Dervan ${ }^{74}$, K. Desch $^{21}$, C. Deterre ${ }^{42}$, P. O. Deviveiros ${ }^{30}$, A. Dewhurst ${ }^{131}$, S. Dhaliwal ${ }^{23}$, A. Di Ciaccio ${ }^{133 a, 133 b}$, L. Di Ciaccio ${ }^{5}$, A. Di Domenico ${ }^{132 a, 132 b}$, C. Di Donato ${ }^{104 a, 104 b}$, A. Di Girolamo ${ }^{30}$, B. Di Girolamo ${ }^{30}$, A. Di Mattia ${ }^{152}$, B. Di Micco ${ }^{134 a, 134 b}$, R. Di Nardo ${ }^{47}$, A. Di Simone ${ }^{48}$, R. Di Sipio ${ }^{158}$, D. Di Valentino ${ }^{29}$, C. Diaconu ${ }^{85}$, M. Diamond ${ }^{158}$, F. A. Dias ${ }^{46}$, M. A. Diaz ${ }^{32 a}$, E. B. Diehl ${ }^{89}$, J. Dietrich ${ }^{16}$, S. Diglio ${ }^{85}$, A. Dimitrievska ${ }^{13}$, J. Dingfelder ${ }^{21}$, P. Dita ${ }^{26 a}$, S. Dita ${ }^{26 a}$, F. Dittus $^{30}$, F. Djama ${ }^{85}$, T. Djobava ${ }^{51 b}$, J. I. Djuvsland ${ }^{58 a}$, M. A. B. do Vale ${ }^{24 c}$, D. Dobos ${ }^{30}$, M. Dobre ${ }^{26 a}$, C. Doglioni ${ }^{81}$, T. Dohmae ${ }^{155}$, J. Dolejsi ${ }^{129}$, Z. Dolezal ${ }^{129}$, B. A. Dolgoshein ${ }^{98, *}$, M. Donadelli ${ }^{24 d}$, S. Donati ${ }^{124 a, 124 b}$, P. Dondero ${ }^{121 a, 121 b}$ ，J. Donini ${ }^{34}$, J. Dopke ${ }^{131}$, A. Doria ${ }^{104 a}$, M. T. Dova ${ }^{71}$, A. T. Doyle ${ }^{53}$, E. Drechsler ${ }^{54}$, M. Dris ${ }^{10}$, E. Dubreuil $^{34}$, E. Duchovni ${ }^{172}$, G. Duckeck ${ }^{100}$, O. A. Ducu ${ }^{26 a}, 85$, D. Duda ${ }^{107}$, A. Dudarev ${ }^{30}$, L. Duflot ${ }^{117}$, L. Duguid ${ }^{77}$, M. Dührssen ${ }^{30}$, M. Dunford ${ }^{58 a}$, H. Duran Yildiz ${ }^{4 a}$, M. Düren ${ }^{52}$, A. Durglishvili51b, D. Duschinger ${ }^{44}$, M. Dyndal ${ }^{38 a}$, C. Eckardt ${ }^{42}$, K. M. Ecker ${ }^{101}$, R. C. Edgar ${ }^{89}$, W. Edson ${ }^{2}$, N. C. Edwards ${ }^{46}$, W. Ehrenfeld ${ }^{21}$, T. Eifert ${ }^{30}$, G. Eigen ${ }^{14}$, K. Einsweiler ${ }^{15}$, T. Ekelof ${ }^{166}$, M. El Kacimi ${ }^{135 c}$, M. Ellert ${ }^{166}$, S. Elles ${ }^{5}$, F. Ellinghaus ${ }^{175}$, A. A. Elliot ${ }^{169}$, N. Ellis ${ }^{30}$, J. Elmsheuser ${ }^{100}$, M. Elsing ${ }^{30}$, D. Emeliyanov ${ }^{131}$, Y. Enari ${ }^{155}$, O. C. Endner ${ }^{83}$, M. Endo ${ }^{118}$, J. Erdmann ${ }^{43}$, A. Ereditato ${ }^{17}$, G. Ernis ${ }^{175}$, J. Ernst ${ }^{2}$, M. Ernst ${ }^{25}$, S. Errede ${ }^{165}$, E. Ertel ${ }^{83}$, M. Escalier ${ }^{117}$, H. Esch ${ }^{43}$, C. Escobar ${ }^{125}$, B. Esposito ${ }^{47}$, A. I. Etienvre ${ }^{136}$, E. Etzion ${ }^{153}$, H. Evans ${ }^{61}$, A. Ezhilov ${ }^{123}$ ， L. Fabbri' ${ }^{20 a, 20 b}$, G. Facini ${ }^{31}$, R. M. Fakhrutdinov ${ }^{130}$, S. Falciano ${ }^{132 a}$ ， R. J. Falla ${ }^{78}$, J. Faltova ${ }^{129}$, Y. Fang ${ }^{33 a}$, M. Fanti ${ }^{91 a, 91 b}$, A. Farbin ${ }^{8}$, A. Farilla ${ }^{134 a}$, T. Farooque ${ }^{12}$, S. Farrel1 ${ }^{15}$, S. M. Farrington ${ }^{170}$, P. Farthouat ${ }^{30}$, F. Fassi ${ }^{135 e}$, P. Fassnacht ${ }^{30}$, D. Fassouliotis ${ }^{9}$, M. Faucci Giannelli ${ }^{77}$,

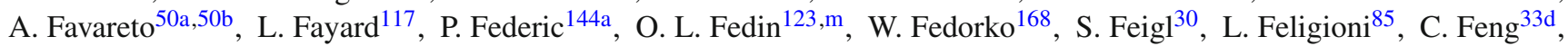


E. J. Feng ${ }^{6}$, H. Feng ${ }^{89}$, A. B. Fenyuk ${ }^{130}$, L. Feremenga ${ }^{8}$, P. Fernandez Martinez ${ }^{167}$, S. Fernandez Perez ${ }^{30}$, J. Ferrando ${ }^{53}$,

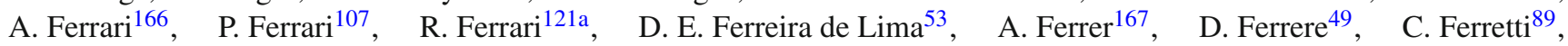
A. Ferretto Parodi ${ }^{50 a, 50 b}$, M. Fiascaris ${ }^{31}$, F. Fiedler ${ }^{83}$, A. Filipčic ${ }^{75}$, M. Filipuzzi ${ }^{42}$, F. Filthaut ${ }^{106}$, M. Fincke-Keeler ${ }^{169}$, K. D. Finelli1 ${ }^{150}$, M. C. N. Fiolhais ${ }^{126 a, 126 c}$, L. Fiorini ${ }^{167}$, A. Firan ${ }^{40}, \quad$ A. Fischer ${ }^{2}, \quad$ C. Fischer ${ }^{12}$, J. Fischer ${ }^{175}$, W. C. Fisher ${ }^{90}$, E. A. Fitzgerald ${ }^{23}$, N. Flaschel ${ }^{42}$, I. Fleck ${ }^{141}$, P. Fleischmann ${ }^{89}$, S. Fleischmann ${ }^{175}$, G. T. Fletcher ${ }^{139}$, G. Fletcher $^{76}$, R. R. M. Fletcher ${ }^{122}$, T. Flick ${ }^{175}$, A. Floderus ${ }^{81}$, L. R. Flores Castillo ${ }^{60 a}$, M. J. Flowerdew ${ }^{101}$, A. Formica ${ }^{136}$, A. Forti ${ }^{84}$, D. Fournier ${ }^{117}$, H. Fox ${ }^{72}$, S. Fracchia ${ }^{12}$, P. Francavilla ${ }^{80}$, M. Franchini ${ }^{20 a, 20 b}$, D. Francis ${ }^{30}$, L. Franconi ${ }^{119}$,

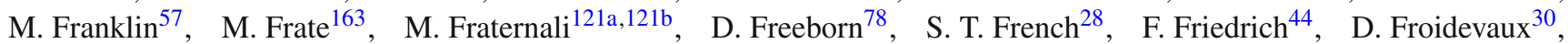
J. A. Frost ${ }^{120}$, C. Fukunaga ${ }^{156}$, E. Fullana Torregrosa ${ }^{83}$, B. G. Fulsom ${ }^{143}$, T. Fusayasu ${ }^{102}$, J. Fuster ${ }^{167}$, C. Gabaldon ${ }^{55}$, O. Gabizon ${ }^{175}$, A. Gabrielli ${ }^{20 a, 20 b}$, A. Gabrielli ${ }^{132 a, 132 b}$, G. P. Gach $^{38 a}$, S. Gadatsch ${ }^{107}$, S. Gadomski ${ }^{49}$, G. Gagliardi ${ }^{50 a, 50 b}$, P. Gagnon ${ }^{61}$, C. Galea ${ }^{106}$, B. Galhardo ${ }^{126 a, 126 c}$, E. J. Gallas ${ }^{120}$, B. J. Gallop ${ }^{131}$, P. Gallus ${ }^{128}$, G. Galster ${ }^{36}$, K. K. Gan ${ }^{111}$, J. Gao ${ }^{33 b, 85}$, Y. Gao ${ }^{46}$, Y. S. Gao ${ }^{143, \text { e, F. M. Garay Walls }}{ }^{46}$, F. Garberson ${ }^{176}$, C. García ${ }^{167}$, J. E. García Navarro ${ }^{167}$, M. Garcia-Sciveres ${ }^{15}$, R. W. Gardner ${ }^{31}$ ， N. Garelli ${ }^{143}$, V. Garonne ${ }^{119}$ ，C. Gatti ${ }^{47}$ ，A. Gaudiello ${ }^{50 a, 50 b}$, G. Gaudio ${ }^{121 a}$, B. Gaur ${ }^{141}$, L. Gauthier ${ }^{95}$, P. Gauzzi1 ${ }^{132 a, 132 b}$, I. L. Gavrilenko ${ }^{96}$, C. Gay ${ }^{168}$, G. Gaycken ${ }^{21}$, E. N. Gazis ${ }^{10}$, P. Ge ${ }^{33 d}$, Z. Gecse ${ }^{168}$, C. N. P. Gee ${ }^{131}$, D. A. A. Geerts ${ }^{107}$, Ch. Geich-Gimbel ${ }^{21}$, M. P. Geisler ${ }^{58 a}$, C. Gemme ${ }^{50 a}$, M. H. Genest ${ }^{55}$, S. Gentile ${ }^{132 a, 132 b}$, M. George ${ }^{54}$, S. George ${ }^{77}$, D. Gerbaudo ${ }^{163}$, A. Gershon ${ }^{153}$, S. Ghasemi ${ }^{141}$, H. Ghazlane ${ }^{135 b}$, B. Giacobbe ${ }^{20 a}$, S. Giagu 132a,132b, V. Giangiobbe ${ }^{12}$, P. Giannetti ${ }^{124 a, 124 b}$, B. Gibbard ${ }^{25}$, S. M. Gibson ${ }^{77}$, M. Gilchriese ${ }^{15}$, T. P. S. Gillam ${ }^{28}$, D. Gillberg ${ }^{30}$, G. Gilles ${ }^{34}$, D. M. Gingrich ${ }^{3, d}$, N. Giokaris ${ }^{9}$, M. P. Giordani ${ }^{164 a, 164 c}$, F. M. Giorgi ${ }^{20 a}$, F. M. Giorgi ${ }^{16}$, P. F. Giraud ${ }^{136}, \quad$ P. Giromini ${ }^{47}, \quad$ D. Giugni ${ }^{91 a}, \quad$ C. Giuliani ${ }^{48}, \quad$ M. Giulini ${ }^{58 b}, \quad$ B. K. Gjelsten ${ }^{119}$, S. Gkaitatzis ${ }^{154}$, I. Gkialas ${ }^{154}$, E. L. Gkougkousis ${ }^{117}$, L. K. Gladilin 99 , C. Glasman ${ }^{82}$, J. Glatzer ${ }^{30}$, P. C. F. Glaysher ${ }^{46}$, A. Glazov ${ }^{42}$, M. Goblirsch-Kolb ${ }^{101}$, J. R. Goddard ${ }^{76}$, J. Godlewski ${ }^{39}$, S. Goldfarb ${ }^{89}$, T. Golling 49, D. Golubkov ${ }^{130}$, A. Gomes ${ }^{126 a, 126 b, 126 d}$, R. Gonçalo ${ }^{126 a}$, J. Goncalves Pinto Firmino Da Costa ${ }^{136}$, L. Gonella ${ }^{21}$, S. González de la Hoz ${ }^{167}$, G. Gonzalez Parra ${ }^{12}$, S. Gonzalez-Sevilla ${ }^{49}$, L. Goossens ${ }^{30}$, P. A. Gorbounov ${ }^{97}$, H. A. Gordon ${ }^{25}$, I. Gorelov ${ }^{105}$, B. Gorini ${ }^{30}$, E. Gorini ${ }^{73 a}$,73b, A. Gorišek ${ }^{75}$, E. Gornicki ${ }^{39}$, A. T. Goshaw ${ }^{45}$, C. Gössling ${ }^{43}$, M. I. Gostkin ${ }^{65}$, D. Goujdami ${ }^{135 c}$,

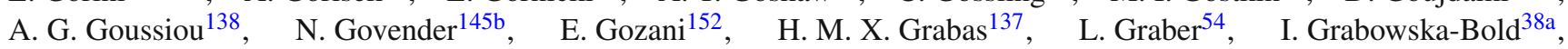
P. O. J. Gradin ${ }^{166}$, P. Grafström ${ }^{20 a, 20 b}$, K-J. Grahn ${ }^{42}$, J. Gramling ${ }^{49}$, E. Gramstad ${ }^{119}$, S. Grancagnolo ${ }^{16}$, V. Grassi ${ }^{148}$, V. Gratchev ${ }^{123}$, H. M. Gray ${ }^{30}$, E. Graziani ${ }^{134 a}$, Z. D. Greenwood ${ }^{79, n}$, K. Gregersen ${ }^{78}$, I. M. Gregor ${ }^{42}$, P. Grenier ${ }^{143}$, J. Griffiths ${ }^{8}$, A. A. Grillo ${ }^{137}$, K. Grimm ${ }^{72}$, S. Grinstein ${ }^{12, o}$, Ph. Gris ${ }^{34}$, J.-F. Grivaz ${ }^{117}$, J. P. Grohs ${ }^{44}$, A. Grohsjean ${ }^{42}$, E. Gross ${ }^{172}$, J. Grosse-Knetter ${ }^{54}$, G. C. Grossi ${ }^{79}$, Z. J. Grout ${ }^{149}$, L. Guan ${ }^{89}$, J. Guenther ${ }^{128}$, F. Guescini ${ }^{49}$, D. Guest ${ }^{176}$, O. Gueta ${ }^{153}$, E. Guido ${ }^{50 a, 50 b}$, T. Guillemin ${ }^{117}, \quad$ S. Guindon ${ }^{2}, \quad$ U. Gul ${ }^{53}, \quad$ C. Gumpert ${ }^{44}, \quad$ J. Guo ${ }^{33 e}$, Y. Guo ${ }^{33 b}$, S. Gupta ${ }^{120}$, G. Gustavino ${ }^{132 a, 132 b}$, P. Gutierrez ${ }^{113}$, N. G. Gutierrez Ortiz ${ }^{78}$, C. Gutschow ${ }^{44}$, C. Guyot ${ }^{136}$, C. Gwenlan ${ }^{120}$, C. B. Gwilliam ${ }^{74}$, A. Haas ${ }^{110}$, C. Haber ${ }^{15}$, H. K. Hadavand ${ }^{8}$, N. Haddad ${ }^{135 e}$, P. Haefnerer1, S. Hageböck ${ }^{21}$, Z. Hajduk ${ }^{39}$, H. Hakobyan ${ }^{177}$, M. Haleem ${ }^{42}$, J. Haley ${ }^{114}$, D. Hall ${ }^{120}$, G. Halladjian ${ }^{90}$, G. D. Hallewell ${ }^{85}$, K. Hamacher ${ }^{175}$, P. Hamal ${ }^{115}$, K. Hamano ${ }^{169}$, M. Hamer ${ }^{54}$, A. Hamilton ${ }^{145 a}$, G. N. Hamity ${ }^{145 c}$, P. G. Hamnett ${ }^{42}$, L. Han ${ }^{33 b}$, K. Hanagaki ${ }^{66, p}$, K. Hanawa ${ }^{155}$, M. Hance ${ }^{15}$, P. Hanke ${ }^{58 a}$, R. Hanna ${ }^{136}$, J. B. Hansen ${ }^{36}$, J. D. Hansen ${ }^{36}$, M. C. Hansen ${ }^{21}$, P. H. Hansen ${ }^{36}$, K. Hara ${ }^{160}$, A. S. Hard ${ }^{173}$, T. Harenberg ${ }^{175}$, F. Hariri ${ }^{117}$, S. Harkusha ${ }^{92}$, R. D. Harrington ${ }^{46}$, P. F. Harrison ${ }^{170}$, F. Hartjes ${ }^{107}$, M. Hasegawa ${ }^{67}$, S. Hasegawa ${ }^{103}$, Y. Hasegawa ${ }^{140}$, A. Hasib ${ }^{113}$, S. Hassani ${ }^{136}$, S. Haug ${ }^{17}$, R. Hauser ${ }^{90}$, L. Hauswald ${ }^{44}$, M. Havranek ${ }^{127}$, C. M. Hawkes ${ }^{18}$, R. J. Hawkings ${ }^{30}$, A. D. Hawkins ${ }^{81}$, T. Hayashi ${ }^{160}$, D. Hayden ${ }^{90}$, C. P. Hays ${ }^{120}$,

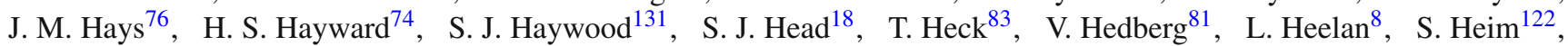
T. Heim ${ }^{175}$, B. Heinemann ${ }^{15}$, L. Heinrich ${ }^{110}$, J. Hejbal ${ }^{127}$, L. Helary ${ }^{22}$, S. Hellman ${ }^{146 a, 146 b}$, D. Hellmich ${ }^{21}$, C. Helsens ${ }^{12}$, J. Henderson ${ }^{120}$, R. C. W. Henderson ${ }^{72}$, Y. Heng ${ }^{173}$, C. Hengler ${ }^{42}$, A. Henrichs ${ }^{176}$, A. M. Henriques Correia ${ }^{30}$, S. Henrot-Versille ${ }^{117}$, G. H. Herbert ${ }^{16}$, Y. Hernández Jiménez ${ }^{167}$, R. Herrberg-Schubert ${ }^{16}$, G. Herten ${ }^{48}$, R. Hertenberger ${ }^{100}$, L. Hervas ${ }^{30}$ ， G. G. Hesketh ${ }^{78}$ ， N. P. Hessey ${ }^{107}$ ， J. W. Hetherly ${ }^{40}$ ，R. Hickling ${ }^{76}$ ， E. Higón-Rodriguez ${ }^{167}$, E. Hill ${ }^{169}$, J. C. Hill ${ }^{28}$, K. H. Hiller ${ }^{42}$, S. J. Hillier ${ }^{18}$, I. Hinchliffe ${ }^{15}$, E. Hines ${ }^{122}$, R. R. Hinman ${ }^{15}$, M. Hirose ${ }^{157}$, D. Hirschbuehl ${ }^{175}$, J. Hobbs ${ }^{148}$, N. Hod ${ }^{107}$, M. C. Hodgkinson ${ }^{139}$, P. Hodgson ${ }^{139}$, A. Hoecker ${ }^{30}$, M. R. Hoeferkamp ${ }^{105}$, F. Hoenig ${ }^{100}$, M. Hohlfeld ${ }^{83}$, D. Hohn ${ }^{21}$, T. R. Holmes ${ }^{15}$, M. Homann ${ }^{43}$, T. M. Hong ${ }^{125}$, L. Hooft van Huysduynen ${ }^{110}$, W. H. Hopkins ${ }^{116}$, Y. Horii ${ }^{103}$, A. J. Horton ${ }^{142}$, J-Y. Hostachy ${ }^{55}$, S. Hou ${ }^{151}$, A. Hoummada ${ }^{135 a}$, J. Howard ${ }^{120}$, J. Howarth ${ }^{42}$, M. Hrabovsky ${ }^{115}$, I. Hristova ${ }^{16}$, J. Hrivnac ${ }^{117}$, T. Hryn'ova ${ }^{5}$, A. Hrynevich ${ }^{93}$, C. Hsu ${ }^{145 c}, \quad$ P. J. Hsu ${ }^{151, q}$, S.-C. Hsu ${ }^{138}, \quad$ D. Hu ${ }^{35}$, Q. Hu ${ }^{33 b}$, X. Hu ${ }^{89}$, Y. Huang ${ }^{42}$ ， Z. Hubacek ${ }^{128}$, F. Hubaut ${ }^{85}$, F. Huegging ${ }^{21}$, T. B. Huffman ${ }^{120}$, E. W. Hughes ${ }^{35}$,

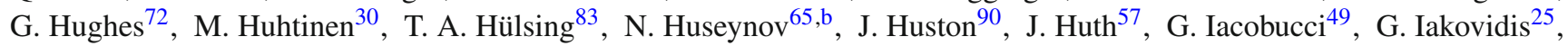
I. Ibragimov $^{141}$, L. Iconomidou-Fayard ${ }^{117}, \quad$ E. Ideal ${ }^{176}, \quad$ Z. Idrissi ${ }^{135 e}, \quad$ P. Iengo ${ }^{30}, \quad$ O. Igonkina ${ }^{107}, \quad$ T. Iizawa $^{171}$, Y. Ikegami $^{66}$, K. Ikematsu ${ }^{141}$, M. Ikeno ${ }^{66}$, Y. Ilchenko ${ }^{31, r}$, D. Iliadis ${ }^{154}$, N. Ilic ${ }^{143}$, T. Ince ${ }^{101}$, G. Introzzi ${ }^{121 a, 121 b}$, P. Ioannou ${ }^{9}$, M. Iodice ${ }^{134 a}$, K. Iordanidou ${ }^{35}$, V. Ippolito ${ }^{57}$, A. Irles Quiles ${ }^{167}$, C. Isaksson ${ }^{166}$, M. Ishino ${ }^{68}$, M. Ishitsuka ${ }^{157}$, 


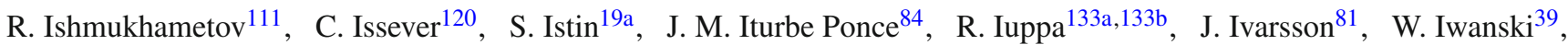
H. Iwasaki ${ }^{66}$ ，J. M. Izen ${ }^{41}$ ，V. Izzo ${ }^{104 a}$ ，S. Jabbar ${ }^{3}$ ，B. Jackson ${ }^{122}$ ， M. Jackson ${ }^{74}$ ，P. Jackson ${ }^{1}$ ，M. R. Jaekel ${ }^{30}$, V. Jain²,

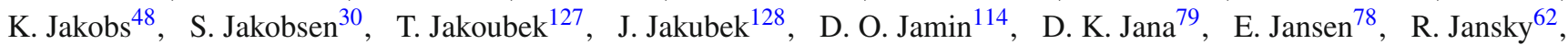
J. Janssen ${ }^{21}$ ， M. Janus ${ }^{170}$ ， G. Jarlskog ${ }^{81}$ ， N. Javadov ${ }^{65, b}$ ， T. Javůrek ${ }^{48}$ ， L. Jeanty ${ }^{15}$ ， J. Jejelava ${ }^{51 a, s}$ ， G.-Y. Jeng ${ }^{150}$, D. Jennens ${ }^{88}$, P. Jenni ${ }^{48, t}$, J. Jentzsch ${ }^{43}$, C. Jeske ${ }^{170}$, S. Jézéquel ${ }^{5}$ ， H. Ji ${ }^{173}$ ， J. Jia ${ }^{148}$, Y. Jiang ${ }^{33 b}, \quad$ S. Jiggins ${ }^{78}$,

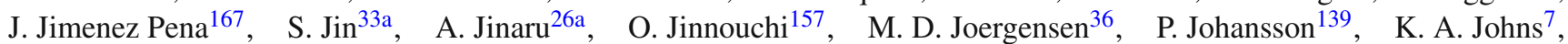
K. Jon-And ${ }^{146 a, 146 b}$ ， G. Jones ${ }^{170}$ ， R. W. L. Jones ${ }^{72}$ ，T. J. Jones ${ }^{74}$ ， J. Jongmanns ${ }^{58 a}$ ，P. M. Jorge ${ }^{126 a, 126 b}$ ， K. D. Joshi ${ }^{84}$, J. Jovicevic ${ }^{159 a}$ ，X. Ju ${ }^{173} ，$ C. A. Jung ${ }^{43}$ ，P. Jussel ${ }^{62}$ ，A. Juste Rozas ${ }^{12, o} ，$ M. Kaci ${ }^{167} ，$ A. Kaczmarska ${ }^{39} ，$ M. Kado ${ }^{117}$, H. Kagan ${ }^{111}$, M. Kagan ${ }^{143}$, S. J. Kahn ${ }^{85}$, E. Kajomovitz ${ }^{45}$, C. W. Kalderon ${ }^{120}$, S. Kama ${ }^{40}$ ， A. Kamenshchikov ${ }^{130}$, N. Kanaya ${ }^{155}$, S. Kaneti ${ }^{28}$, V. A. Kantserov ${ }^{98}$, J. Kanzaki ${ }^{66}$, B. Kaplan ${ }^{110}$, L. S. Kaplan ${ }^{173}$, A. Kapliy ${ }^{31}$, D. Kar ${ }^{53}$, K. Karakostas ${ }^{10}$, A. Karamaoun ${ }^{3}$, N. Karastathis ${ }^{10,107}$, M. J. Kareem ${ }^{54}$, E. Karentzos ${ }^{10}$, M. Karnevskiy ${ }^{83}$, S. N. Karpov ${ }^{65}$, Z. M. Karpova ${ }^{65}$, K. Karthik ${ }^{110}$, V. Kartvelishvili ${ }^{72}$, A. N. Karyukhin ${ }^{130}$, L. Kashif ${ }^{173}$, R. D. Kass ${ }^{111}$, A. Kastanas ${ }^{14}$, Y. Kataoka ${ }^{155}$, A. Katre ${ }^{49}$, J. Katzy ${ }^{42}$, K. Kawagoe ${ }^{70}$, T. Kawamoto ${ }^{155}$, G. Kawamura ${ }^{54}$, S. Kazama ${ }^{155}$, V. F. Kazanin ${ }^{109, c}$, $\begin{array}{llll}\text { R. Keeler } & 169 & \text { R. Kehoe } & 40\end{array}$ J. S. Keller ${ }^{42}$, J. J. Kempster ${ }^{77}$, H. Keoshkerian ${ }^{84}$, O. Kepka ${ }^{127}$, B. P. Kerševan ${ }^{75}$,

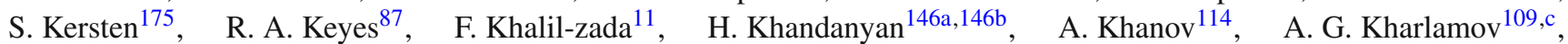
T. J. Khoo ${ }^{28}$, V. Khovanskiy ${ }^{97}$, E. Khramov ${ }^{65}$, J. Khubua ${ }^{51 b, u}$, H. Y. Kim ${ }^{8}$, H. Kim ${ }^{146 a, 146 b}$, S. H. Kim ${ }^{160}$, Y. Kim${ }^{31}$, N. Kimura ${ }^{154}$, O. M. Kind ${ }^{16}$, B. T. King ${ }^{74}$, M. King ${ }^{167}$, S. B. King ${ }^{168}$, J. Kirk ${ }^{131}$, A. E. Kiryunin ${ }^{101}$, T. Kishimoto ${ }^{67}$, D. Kisielewska ${ }^{38 a}$, F. Kiss ${ }^{48}$, K. Kiuchi ${ }^{160}$, O. Kivernyk ${ }^{136}$, E. Kladiva ${ }^{144 b}$, M. H. Klein ${ }^{35}$, M. Klein ${ }^{74}$, U. Klein ${ }^{74}$, K. Kleinknecht ${ }^{83}$, P. Klimek ${ }^{146 a, 146 b}$, A. Klimentov ${ }^{25}$, R. Klingenberg ${ }^{43}$, J. A. Klinger ${ }^{139}$, T. Klioutchnikova ${ }^{30}$, E.-E. Kluge ${ }^{58 a}$, P. Kluit ${ }^{107}$, S. Kluth ${ }^{101}$, J. Knapik ${ }^{39}$, E. Kneringer ${ }^{62}$, E. B. F. G. Knoops ${ }^{85}$, A. Knue ${ }^{53}$, A. Kobayashi ${ }^{155}$, D. Kobayashi ${ }^{157}$, T. Kobayashi ${ }^{155}$, M. Kobel ${ }^{44}$, M. Kocian ${ }^{143}$, P. Kodys ${ }^{129}$, T. Koffas ${ }^{29}$, E. Koffeman ${ }^{107}$, L. A. Kogan ${ }^{120}$, S. Kohlmann ${ }^{175}$, Z. Kohout ${ }^{128}$, T. Kohriki ${ }^{66}$, T. Koi ${ }^{143}$, H. Kolanoski ${ }^{16}$, I. Koletsou ${ }^{5}$, A. A. Komar ${ }^{96,{ }^{*}}$, Y. Komori ${ }^{155}$, T. Kondo ${ }^{66}$, N. Kondrashova ${ }^{42}$, K. Köneke ${ }^{48}$, A. C. König ${ }^{106}$, T. Kono ${ }^{66}$, R. Konoplich ${ }^{110, v}$, N. Konstantinidis ${ }^{78}$, R. Kopeliansky ${ }^{152}$, S. Koperny ${ }^{38 a}$, L. Köpke ${ }^{83}$, A. K. Kopp ${ }^{48}$, K. Korcyl ${ }^{39}$, K. Kordas ${ }^{154}$, A. Korn ${ }^{78}$, A. A. Korol ${ }^{109, c}$,

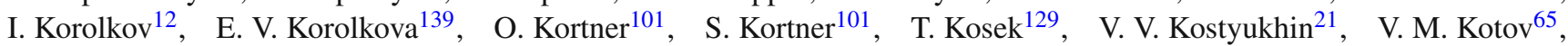
A. Kotwal ${ }^{45}$, A. Kourkoumeli-Charalampidi ${ }^{154}$, C. Kourkoumelis ${ }^{9}$, V. Kouskoura ${ }^{25}$, A. Koutsman ${ }^{159 a}$, R. Kowalewski ${ }^{169}$, T. Z. Kowalski ${ }^{38 a}$, W. Kozanecki ${ }^{136}$, A. S. Kozhin ${ }^{130}$, V. A. Kramarenko ${ }^{99}$, G. Kramberger ${ }^{75}$, D. Krasnopevtsev ${ }^{98}$, M. W. Krasny ${ }^{80}$, A. Krasznahorkay ${ }^{30}$, J. K. Kraus ${ }^{21}$, A. Kravchenko ${ }^{25}$, S. Kreiss ${ }^{110}$, M. Kretz ${ }^{58 c}$, J. Kretzschmar ${ }^{74}$, K. Kreutzfeldt ${ }^{52}$, P. Krieger ${ }^{158}$, K. Krizka ${ }^{31}$, K. Kroeninger ${ }^{43}$, H. Kroha ${ }^{101}$, J. Kroll ${ }^{122}$, J. Kroseberg ${ }^{21}$, J. Krstic ${ }^{13}$, U. Kruchonak ${ }^{65}$, H. Krüger ${ }^{21}$, N. Krumnack ${ }^{64}$, A. Kruse ${ }^{173}$, M. C. Kruse ${ }^{45}$, M. Kruskal ${ }^{22}$, T. Kubota ${ }^{88}$, H. Kucuk ${ }^{78}$, S. Kuday ${ }^{4 b}$, S. Kuehn ${ }^{48}$, A. Kugel ${ }^{58 c}$, F. Kuger ${ }^{174}$, A. Kuhl ${ }^{137}$, T. Kuhl ${ }^{42}$, V. Kukhtin ${ }^{65}$, Y. Kulchitsky ${ }^{92}$, S. Kuleshov ${ }^{32 b}$, M. Kuna ${ }^{132 a, 132 b}$, T. Kunigo ${ }^{68}$, A. Kupco ${ }^{127}$, H. Kurashige ${ }^{67}, \quad$ Y. A. Kurochkin ${ }^{92}$, V. Kus ${ }^{127}$, E. S. Kuwertz ${ }^{169}$, M. Kuze ${ }^{157}$, J. Kvita ${ }^{115}$, T. Kwan ${ }^{169}$, D. Kyriazopoulos ${ }^{139}$, A. La Rosa ${ }^{137}$, J. L. La Rosa Navarro ${ }^{24 d}$, L. La Rotonda ${ }^{37 a, 37 b}$, C. Lacasta ${ }^{167}$, F. Lacava ${ }^{132 a, 132 b}$, J. Lacey ${ }^{29}$, H. Lacker ${ }^{16}$, D. Lacour ${ }^{80}$, V. R. Lacuesta ${ }^{167}$, E. Ladygin ${ }^{65}$, R. Lafaye ${ }^{5}$, B. Laforge ${ }^{80}$, T. Lagouri ${ }^{176}$, S. Lai ${ }^{54}$, L. Lambourne ${ }^{78}$, S. Lammers ${ }^{61}$, C. L. Lampen ${ }^{7}$, W. Lampl ${ }^{7}$, E. Lançon ${ }^{136}$,

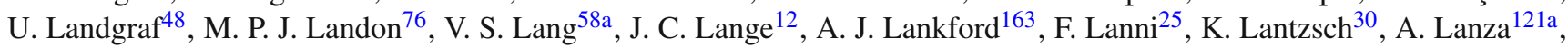
S. Laplace ${ }^{80}$, C. Lapoire $^{30}$, J. F. Laporte ${ }^{136}$, T. Lari ${ }^{11 a}, \quad$ F. Lasagni Manghi ${ }^{20 a, 20 b}$, M. Lassnig ${ }^{30}, \quad$ P. Laurelli ${ }^{47}$, W. Lavrijsen ${ }^{15}$, A. T. Law ${ }^{137}$, P. Laycock ${ }^{74}$, T. Lazovich ${ }^{57}$, O. Le Dortz ${ }^{80}$, E. Le Guirriec ${ }^{85}$, E. Le Menedeu ${ }^{12}$, M. LeBlanc ${ }^{169}$, T. LeCompte ${ }^{6}$, F. Ledroit-Guillon ${ }^{55}$, C. A. Lee ${ }^{145 b}$, S. C. Lee ${ }^{151}$, L. Lee ${ }^{1}$, G. Lefebvre ${ }^{80}$, M. Lefebvre ${ }^{169}$, F. Legger ${ }^{100}$, C. Leggett ${ }^{15}$, A. Lehan ${ }^{74}$, G. Lehmann Miotto $^{30}$, X. Lei ${ }^{7}$, W. A. Leight ${ }^{29}$, A. Leisos ${ }^{154, w}$, A. G. Leister ${ }^{176}$, M. A. L. Leite ${ }^{24 d}$, R. Leitner ${ }^{129}$ ， D. Lellouch ${ }^{172}$, B. Lemmer ${ }^{54}$ ， K. J. C. Leney ${ }^{78}$, T. Lenz ${ }^{21}$, B. Lenzi ${ }^{30}$, R. Leone ${ }^{7}$, S. Leone ${ }^{124 a, 124 b}$, C. Leonidopoulos ${ }^{46}$, S. Leontsinis ${ }^{10}$, G. Lerner ${ }^{149}$, C. Leroy ${ }^{95}$, C. G. Lester ${ }^{28}$, M. Levchenko ${ }^{123}$, J. Levêque ${ }^{5}$, D. Levin ${ }^{89}$, L. J. Levinson ${ }^{172}$, M. Levy ${ }^{18}$, A. Lewis ${ }^{120}$, A. M. Leyko ${ }^{21}$, M. Leyton ${ }^{41}$, B. Li ${ }^{33 b, x}, H^{3}$ Li $^{148}$,

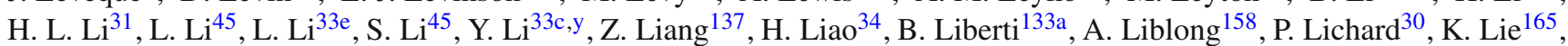

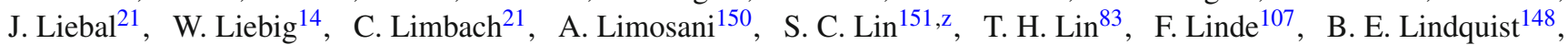
J. T. Linnemann ${ }^{90}$, E. Lipeles ${ }^{122}$, A. Lipniacka ${ }^{14}$, M. Lisovyi ${ }^{58 b}$, T. M. Liss ${ }^{165}$, D. Lissauer ${ }^{25}$, A. Lister ${ }^{168}$, A. M. Litke ${ }^{137}$,

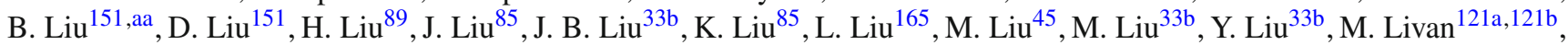
A. Lleres ${ }^{55}$, J. Llorente Merino ${ }^{82}$, S. L. Lloyd ${ }^{76}$, F. Lo Sterzo ${ }^{151}$, E. Lobodzinska ${ }^{42}$, P. Loch ${ }^{7}$, W. S. Lockman ${ }^{137}$, F. K. Loebinger ${ }^{84}$, A. E. Loevschall-Jensen ${ }^{36}$, A. Loginov ${ }^{176}$, T. Lohse ${ }^{16}$, K. Lohwasser ${ }^{42}$, M. Lokajicek ${ }^{127}$, B. A. Long ${ }^{22}$, J. D. Long ${ }^{89}$ ，R. E. Long ${ }^{72}$ ， K. A. Looper ${ }^{111}$ ，L. Lopes ${ }^{126 a ， D . ~ L o p e z ~ M a t e o s ~}{ }^{57}$ ，B. Lopez Paredes ${ }^{139}$ ， I. Lopez Paz ${ }^{12}$, J. Lorenz ${ }^{100}$ ， N. Lorenzo Martinez ${ }^{61}$ ， M. Losada ${ }^{162}$ ，P. Loscutoff ${ }^{15}$ ，P. J. Lösel ${ }^{100}$ ，X. Lou ${ }^{33 a}$ ，A. Lounis ${ }^{117}$ ，J. Love ${ }^{6}$, P. A. Love ${ }^{72}$, N. Lu ${ }^{89}$, H. J. Lubatti ${ }^{138}$, C. Luci ${ }^{132 a, 132 b}$, A. Lucotte ${ }^{55}$, F. Luehring ${ }^{61}$, W. Lukas ${ }^{62}$, L. Luminari ${ }^{132 a}$, O. Lundberg ${ }^{146 a, 146 b}$, B. Lund-Jensen ${ }^{147}$, D. Lynn ${ }^{25}$, R. Lysak ${ }^{127}$, E. Lytken ${ }^{81}$, H. Ma $^{25}$, L. L. Ma ${ }^{33 d}$, G. Maccarrone ${ }^{47}$, 
A. Macchiolo ${ }^{101}$ ， C. M. Macdonald ${ }^{139}$ ， J. Machado Miguens ${ }^{122,126 b}, \quad$ D. Macina ${ }^{30}, \quad$ D. Madaffari ${ }^{85}, \quad$ R. Madar ${ }^{34}$ H. J. Maddocks ${ }^{72}$, W. F. Mader ${ }^{44}$, A. Madsen ${ }^{166}$, S. Maeland ${ }^{14}$, T. Maeno ${ }^{25}$, A. Maevskiy ${ }^{99}$,E. Magradze ${ }^{54}$, K. Mahboubi ${ }^{48}$, J. Mahlstedt ${ }^{107}$, C. Maiani ${ }^{136}$, C. Maidantchik ${ }^{24 a}$, A. A. Maier ${ }^{101}$, T. Maier ${ }^{100}$, A. Maio ${ }^{126 a, 126 b, 126 d}$, S. Majewski ${ }^{116}$, Y. Makida ${ }^{66}$, N. Makovec ${ }^{117}$, B. Malaescu ${ }^{80}$, Pa. Malecki ${ }^{39}$, V.P. Maleev ${ }^{123}$, F. Malek ${ }^{55}$, U. Mallik ${ }^{63}$, D. Malon ${ }^{6}$, C. Malone ${ }^{143}$, S. Maltezos ${ }^{10}$, V. M. Malyshev ${ }^{109}$, S. Malyukov ${ }^{30}$, J. Mamuzic ${ }^{42}$, G. Mancini ${ }^{47}$, B. Mandelli ${ }^{30}$, L. Mandelli ${ }^{91 a}$, I. Mandic ${ }^{75}$, R. Mandrysch ${ }^{63}$, J. Maneira ${ }^{126 a, 126 b}$, A. Manfredini ${ }^{101}$, L. Manhaes de Andrade Filho ${ }^{24 b}$, J. Manjarres Ramos ${ }^{159 b}$, A. Mann ${ }^{100}$, P. M. Manning ${ }^{137}$, A. Manousakis-Katsikakis ${ }^{9}$, B. Mansoulie ${ }^{136}$, R. Mantifel ${ }^{87}$, M. Mantoani ${ }^{54}$, L. Mapelli ${ }^{30}$, L. March ${ }^{145 c}$, G. Marchiori ${ }^{80}$, M. Marcisovsky ${ }^{127}$, C. P. Marino ${ }^{169}$, M. Marjanovic ${ }^{13}$, D. E. Marley ${ }^{89}$ ， F. Marroquim ${ }^{24 a}$, S. P. Marsden ${ }^{84}$, Z. Marshall ${ }^{15}$, L. F. Marti ${ }^{17}$ ， S. Marti-Garcia ${ }^{167}$, B. Martin ${ }^{90}$,

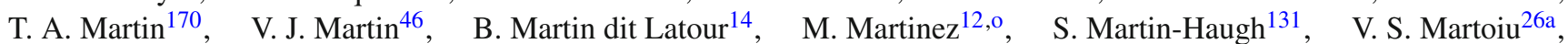
A. C. Martyniuk ${ }^{78}$, M. Marx ${ }^{138}$, F. Marzano ${ }^{132 a}$, A. Marzin $^{30}$, L. Masetti ${ }^{83}$, T. Mashimo ${ }^{155}$, R. Mashinistov ${ }^{96}$, J. Masik ${ }^{84}$,

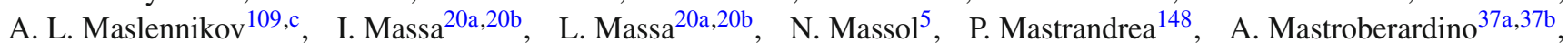
T. Masubuchi ${ }^{155}$, P. Mättig ${ }^{175}$ ， J. Mattmann ${ }^{83}$ ， J. Maurer ${ }^{26 a}$ ， S. J. Maxfield ${ }^{74}$ ， D. A. Maximov ${ }^{109, c}$, R. Mazini ${ }^{151}$, S. M. Mazza ${ }^{91 a, 91 b}$, L. Mazzaferro ${ }^{133 a, 133 b}$, G. Mc Goldrick ${ }^{158}$, S. P. Mc Kee ${ }^{89}$, A. McCarn ${ }^{89}$, R. L. McCarthy ${ }^{148}$, T. G. McCarthy ${ }^{29}$, N. A. McCubbin ${ }^{131}$, K. W. McFarlane ${ }^{56, *}$, J. A. Mcfayden ${ }^{78}$, G. Mchedlidze ${ }^{54}$, S. J. McMahon ${ }^{131}$, R. A. McPherson ${ }^{169, k}, \quad$ M. Medinnis ${ }^{42}$, S. Meehan ${ }^{145 a}$, S. Mehlhase ${ }^{100}$, A. Mehta ${ }^{74}, \quad$ K. Meier ${ }^{58 a}$, C. Meineck ${ }^{100}$,

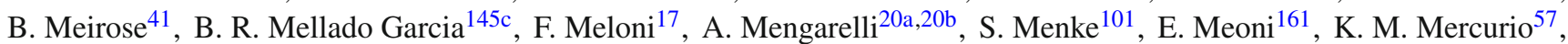
S. Mergelmeyer ${ }^{21}$, P. Mermod ${ }^{49}$, L. Merola ${ }^{104 a, 104 b}$, C. Meroni ${ }^{91 a}$, F. S. Merritt ${ }^{31}$, A. Messina ${ }^{132 a, 132 b}$, J. Metcalfe ${ }^{25}$, A. S. Mete ${ }^{163}$, C. Meyer ${ }^{83}$, C. Meyer ${ }^{122}$, J-P. Meyer ${ }^{136}$, J. Meyer ${ }^{107}$, R. P. Middleton ${ }^{131}$, S. Miglioranzi ${ }^{164 a, 164 c}$, L. Mijović21 ${ }^{21}$ G. Mikenberg ${ }^{172}$, M. Mikestikova ${ }^{127}$, M. Mikuž ${ }^{75}$, M. Milesi ${ }^{88}$, A. Milic ${ }^{30}$, D. W. Miller ${ }^{31}$, C. Mills ${ }^{46}$, A. Milov ${ }^{172}$, D. A. Milstead ${ }^{146 a, 146 b}$, A. A. Minaenko ${ }^{130}$, Y. Minami ${ }^{155}$, I. A. Minashvili65, A. I. Mincer ${ }^{110}$, B. Mindur ${ }^{38 a}$, M. Mineev ${ }^{65}$, Y. Ming ${ }^{173}$, L. M. Mir ${ }^{12}$, T. Mitani ${ }^{171}$, J. Mitrevski ${ }^{100}$, V. A. Mitsou ${ }^{167}$, A. Miucci ${ }^{49}$, P. S. Miyagawa ${ }^{139}$, J. U. Mjörnmark ${ }^{81}$, T. Moa ${ }^{146 a, 146 b}$, K. Mochizuki ${ }^{85}$, S. Mohapatra ${ }^{35}$, W. Mohr ${ }^{48}$, S. Molander 146a,146b, R. Moles-Valls ${ }^{21}$, K. Mönig ${ }^{42}$, C. Monini ${ }^{55}$, J. Monk ${ }^{36}$, E. Monnier ${ }^{85}$, J. Montejo Berlingen ${ }^{12}$, F. Monticelli ${ }^{71}$, S. Monzani ${ }^{132 a, 132 b}$, R. W. Moore ${ }^{3}$, N. Morange ${ }^{117}$, D. Moreno ${ }^{162}$, M. Moreno Llácer ${ }^{54}$, P. Morettini ${ }^{50 a}$, M. Morgenstern ${ }^{44}$, D. Mori ${ }^{142}$,

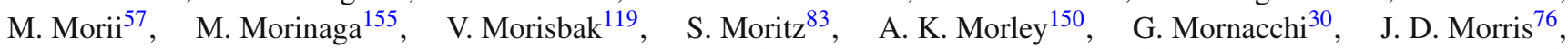
S. S. Mortensen ${ }^{36}$, A. Morton ${ }^{53}$, L. Morvaj ${ }^{103}$, M. Mosidze ${ }^{51 b}$, J. Moss ${ }^{111}$, K. Motohashi ${ }^{157}$, R. Mount ${ }^{143}$, E. Mountricha ${ }^{25}$, S. V. Mouraviev ${ }^{96, *}$, E. J. W. Moyse ${ }^{86}$, S. Muanza ${ }^{85}$, R. D. Mudd ${ }^{18}$, F. Mueller ${ }^{101}$, J. Mueller ${ }^{125}$, R. S. P. Mueller ${ }^{100}$,

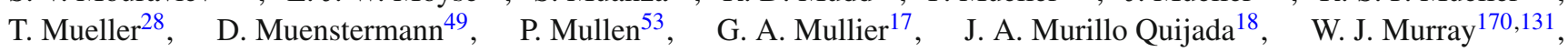
H. Musheghyan ${ }^{54}$, E. Musto ${ }^{152}$, A. G. Myagkov ${ }^{130, a b}$, M. Myska ${ }^{128}$, B. P. Nachman ${ }^{143}$, O. Nackenhorst ${ }^{54}$, J. Nadal ${ }^{54}$, K. Nagai ${ }^{120}$, R. Nagai ${ }^{157}$, Y. Nagai ${ }^{85}$, K. Nagano ${ }^{66}$, A. Nagarkar ${ }^{111}$, Y. Nagasaka ${ }^{59}$, K. Nagata ${ }^{160}$, M. Nagel ${ }^{101}$, E. Nagy ${ }^{85}$, A. M. Nairz ${ }^{30}$, Y. Nakahama ${ }^{30}$, K. Nakamura ${ }^{66}$, T. Nakamura ${ }^{155}$, I. Nakano ${ }^{112}$, H. Namasivayam ${ }^{41}$, R. F. Naranjo Garcia ${ }^{42}$, R. Narayan ${ }^{31}$, T. Naumann ${ }^{42}$, G. Navarro ${ }^{162}$, R. Nayyar ${ }^{7}$, H. A. Neal ${ }^{89}$, P. Yu. Nechaeva ${ }^{96}$, T. J. Neep ${ }^{84}$, P. D. Nef ${ }^{143}$, A. Negri ${ }^{121 a, 121 b}$, M. Negrini ${ }^{20 a}$, S. Nektarijevic ${ }^{106}, \quad$ C. Nellist ${ }^{117}$, A. Nelson ${ }^{163}$, S. Nemecek ${ }^{127}$, P. Nemethy ${ }^{110}$, A. A. Nepomuceno ${ }^{24 a}$, M. Nessi ${ }^{30, a c}$, M. S. Neubauer ${ }^{165}$, M. Neumann ${ }^{175}$, R. M. Neves ${ }^{110}$, P. Nevski ${ }^{25}$, P. R. Newman ${ }^{18}$, D. H. Nguyen ${ }^{6}$, R. B. Nickerson ${ }^{120}$, R. Nicolaidou ${ }^{136}$, B. Nicquevert ${ }^{30}$, J. Nielsen ${ }^{137}$, N. Nikiforou ${ }^{35}$, A. Nikiforov ${ }^{16}$, V. Nikolaenko ${ }^{130, a b}$, I. Nikolic-Audit ${ }^{80}$, K. Nikolopoulos ${ }^{18}$, J. K. Nilsen ${ }^{119}$, P. Nilsson ${ }^{25}$, Y. Ninomiya ${ }^{155}$, A. Nisati ${ }^{132 a}$, R. Nisius ${ }^{101}$, T. Nobe ${ }^{155}$, M. Nomachi ${ }^{118}$, I. Nomidis ${ }^{29}$, T. Nooney ${ }^{76}$, S. Norberg ${ }^{13}$, M. Nordberg ${ }^{30}$, O. Novgorodova ${ }^{44}$, S. Nowak ${ }^{101}$, M. Nozaki ${ }^{66}$, L. Nozka ${ }^{115}$, K. Ntekas ${ }^{10}$, G. Nunes Hanninger ${ }^{88}$, T. Nunnemann ${ }^{100}$, E. Nurse ${ }^{78}$, F. Nuti ${ }^{88}$, $\begin{array}{llll}\text { B. J. O'Brien } & 46 & \text { F. O'grady }\end{array}{ }^{7}$, D. C. O’Neil ${ }^{142}$, V. O'Shea ${ }^{53}$, F. G. Oakham ${ }^{29, d}$, H. Oberlack ${ }^{101}, \quad$ T. Obermann ${ }^{21}$, J. Ocariz ${ }^{80}$, A. Ochi ${ }^{67}$, I. Ochoa ${ }^{78}$, J. P. Ochoa-Ricoux ${ }^{32 a}$, S. Oda ${ }^{70}$, S. Odaka ${ }^{66}$, H. Ogren ${ }^{61}$, A. Oh ${ }^{84}$, S. H. $\mathrm{Oh}^{45}$, C. C. $\mathrm{Ohm}^{15}$, H. Ohman ${ }^{166}$, H. Oide ${ }^{30}$, W. Okamura ${ }^{118}$, H. Okawa ${ }^{160}$, Y. Okumura ${ }^{31}$, T. Okuyama ${ }^{66}$, A. Olariu ${ }^{26 a}$, S. A. Olivares Pino ${ }^{46}$, D. Oliveira Damazio ${ }^{25}$, E. Oliver Garcia ${ }^{167}$, A. Olszewski ${ }^{39}$, J. Olszowska ${ }^{39}$, A. Onofre ${ }^{126 a, 126 e}$, P. U. E. Onyisi ${ }^{31, r}$, C. J. Oram ${ }^{159 a}$, M. J. Oreglia ${ }^{31}$, Y. Oren ${ }^{153}$, D. Orestano ${ }^{134 a, 134 b}$, N. Orlando ${ }^{154}$, C. Oropeza Barrera ${ }^{53}$, R. S. Orr ${ }^{158}$, B. Osculati ${ }^{50 a, 50 b}$, R. Ospanov ${ }^{84}$, G. Otero y Garzon ${ }^{27}$, H. Otono ${ }^{70}$, M. Ouchrif ${ }^{135 d}$, E. A. Ouellette ${ }^{169}$, F. Ould-Saada ${ }^{119}$, A. Ouraou ${ }^{136}$, K. P. Oussoren ${ }^{107}$, Q. Ouyang ${ }^{33 a}$, A. Ovcharova ${ }^{15}$, M. Owen ${ }^{53}$, R. E. Owen ${ }^{18}$, V. E. Ozcan ${ }^{19 a}$, N. Ozturk ${ }^{8}$, K. Pachal ${ }^{142}$, A. Pacheco Pages ${ }^{12}$, C. Padilla Aranda ${ }^{12}$, M. Pagáčová ${ }^{48}$, S. Pagan Griso ${ }^{15}$,

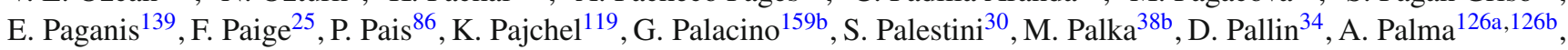

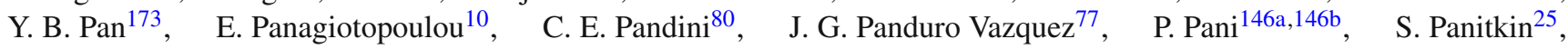
D. Pantea ${ }^{26 a}$, L. Paolozzi ${ }^{49}$, Th. D. Papadopoulou ${ }^{10}$, K. Papageorgiou ${ }^{154}$, A. Paramonov ${ }^{6}$, D. Paredes Hernandez ${ }^{154}$, M. A. Parker $^{28}$, K. A. Parker $^{139}$, F. Parodi ${ }^{50,50 b}$, J. A. Parsons ${ }^{35}$, U. Parzefall ${ }^{48}$, E. Pasqualucci ${ }^{132 a}$, S. Passaggio ${ }^{50 a}$, F. Pastore ${ }^{134 a, 134 b, *}$, Fr. Pastore 77 , G. Pásztor ${ }^{29}$, S. Pataraia ${ }^{175}$, N. D. Patel ${ }^{150}$, J. R. Pater ${ }^{84}$, T. Pauly ${ }^{30}$, J. Pearce ${ }^{169}$, B. Pearson ${ }^{113}$, L. E. Pedersen ${ }^{36}$, M. Pedersen ${ }^{119}, \quad$ S. Pedraza Lopez ${ }^{167}$, R. Pedro ${ }^{126 a, 126 b}, \quad$ S. V. Peleganchuk ${ }^{109, c}$, 
D. Pelikan ${ }^{166}$, O. Penc ${ }^{127}$, C. Peng ${ }^{33 a}$, H. Peng ${ }^{33 b}$, B. Penning ${ }^{31}$, J. Penwell ${ }^{61}$, D. V. Perepelitsa ${ }^{25}$, E. Perez Codina ${ }^{159 a}$, M. T. Pérez García-Estañ ${ }^{167}$, L. Perini ${ }^{91 a, 91 b}$, H. Pernegger ${ }^{30}$, S. Perrella ${ }^{104 a, 104 b}$, R. Peschke ${ }^{42}$, V. D. Peshekhonov ${ }^{65}$, K. Peters ${ }^{30}$, R. F. Y. Peters ${ }^{84}$, B. A. Petersen ${ }^{30}$, T. C. Petersen ${ }^{36}$, E. Petit ${ }^{42}$, A. Petridis ${ }^{146 a, 146 b}$, C. Petridou ${ }^{154}$, P. Petroff $^{117}$, E. Petrolo ${ }^{132 a}$, F. Petrucci134a,134b , N.E. Pettersson ${ }^{157}$, R. Pezoa ${ }^{32 b}$, P. W. Phillips ${ }^{131}$, G. Piacquadio ${ }^{143}$, E. Pianori ${ }^{170}$,

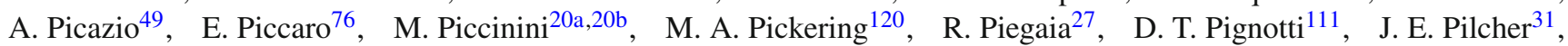
A. D. Pilkington ${ }^{84}$, J. Pina ${ }^{126 a, 126 b, 126 d}$, M. Pinamonti ${ }^{164 a, 164 c, a d}$, J. L. Pinfold ${ }^{3}$, A. Pingel ${ }^{36}$, B. Pinto ${ }^{126 a}$, S. Pires ${ }^{80}$, H. Pirumov ${ }^{42}$, M. Pitt ${ }^{172}$, C. Pizio ${ }^{91 a, 91 b}$, L. Plazak ${ }^{144 a}$, M.-A. Pleier ${ }^{25}$, V. Pleskot ${ }^{129}$, E. Plotnikova ${ }^{65}$, P. Plucinski ${ }^{146 a, 146 b}$, D. Pluth ${ }^{64}$, R. Poettgen ${ }^{146 a, 146 b}$, L. Poggioli ${ }^{117}, \quad$ D. Pohl ${ }^{21}$, G. Polesello ${ }^{121 a}$, A. Poley ${ }^{42}$ A. Policicchio ${ }^{37 a, 37 b}$, R. Polifka ${ }^{158}$, A. Polini ${ }^{20 a}$, C. S. Pollard ${ }^{33}$, V. Polychronakos ${ }^{25}$, K. Pommès ${ }^{30}$, L. Pontecorvo ${ }^{132 a}$, B. G. Pope ${ }^{90}$, G. A. Popeneciu ${ }^{26 b}$, D. S. Popovic ${ }^{13}$, A. Poppleton ${ }^{30}$, S. Pospisil ${ }^{128}$, K. Potamianos ${ }^{15}$, I. N. Potrap ${ }^{65}$, C. J. Potter ${ }^{149}$, C. T. Potter ${ }^{116}$, G. Poulard ${ }^{30}$, J. Poveda ${ }^{30}$, V. Pozdnyakov ${ }^{65}$, P. Pralavorio ${ }^{85}$, A. Pranko ${ }^{15}$, S. Prasad ${ }^{30}$, S. Prell ${ }^{64}$, D. Price ${ }^{84}$, L. E. Price ${ }^{6}$, M. Primavera ${ }^{73 a}$, S. Prince ${ }^{87}$, M. Proissl ${ }^{46}$, K. Prokofiev ${ }^{60 c}$, F. Prokoshin ${ }^{32 b}$, E. Protopapadaki ${ }^{136}$, S. Protopopescu ${ }^{25}$, J. Proudfoot ${ }^{6}$, M. Przybycien ${ }^{38 a}$, E. Ptacek ${ }^{116}$, D. Puddu ${ }^{134 a, 134 b}$, E. Pueschel ${ }^{86}$, D. Puldon ${ }^{148}$, M. Purohit ${ }^{25}$,ae , P. Puzo ${ }^{117}$, J. Qian ${ }^{89}$, G. Qin ${ }^{53}$, Y. Qin ${ }^{84}$, A. Quadt ${ }^{54}$, D. R. Quarrie ${ }^{15}$, W. B. Quayle ${ }^{164 a, 164 b}$, M. Queitsch-Maitland ${ }^{84}$, D. Quilty ${ }^{53}$, S. Raddum ${ }^{119}$, V. Radeka ${ }^{25}$, V. Radescu ${ }^{42}$, S. K. Radhakrishnan ${ }^{148}$, P. Radloff ${ }^{116}$, P. $\operatorname{Rados}^{88}$, F. Ragusa ${ }^{91 a}$,91b, G. Rahal ${ }^{178}$, S. Rajagopalan ${ }^{25}$, M. Rammensee ${ }^{30}$, C. Rangel-Smith ${ }^{166}$, F. Rauscher ${ }^{100}$, S. $\operatorname{Rave}^{83}$, T. Ravenscroft ${ }^{53}$, M. Raymond ${ }^{30}$, A. L. $\operatorname{Read}^{119}$, N. P. Readioff ${ }^{74}$, D. M. Rebuzzi ${ }^{121 a, 121 b}$, A. Redelbach ${ }^{174}$, G. Redlinger $^{25}$, R. Reece ${ }^{137}$, K. Reeves ${ }^{41}$, L. Rehnisch ${ }^{16}$, J. Reichert ${ }^{122}$, H. Reisin ${ }^{27}$, M. Relich ${ }^{163}$, C. Rembser ${ }^{30}$, H. Ren $^{33 a}$, A. Renaud ${ }^{117}$, M. Rescigno ${ }^{132 a}$, S. Resconi ${ }^{91 a}$, O. L. Rezanova ${ }^{109, c}, \quad$ P. Reznicek ${ }^{129}$, R. Rezvani ${ }^{95}$, R. Richter $^{101}$, S. Richter ${ }^{78}$, E. Richter-Was ${ }^{38 b}$, O. Ricken ${ }^{21}$, M. Ridel ${ }^{80}$, P. Rieck ${ }^{16}$, C. J. Riegel ${ }^{175}$, J. Rieger ${ }^{54}$, M. Rijssenbeek ${ }^{148}$, A. Rimoldi121a,121b, L. Rinaldi ${ }^{20 a}$, B. Ristićc9 ${ }^{49}$ E. Ritsch ${ }^{30}$, I. Riu ${ }^{12}$, F. Rizatdinova ${ }^{114}$, E. Rizvi ${ }^{76}$, S. H. Robertson ${ }^{87, k}$, A. Robichaud-Veronneau ${ }^{87}$, D. Robinson ${ }^{28}$, J. E. M. Robinson ${ }^{42}$, A. Robson ${ }^{53}$, C. Roda ${ }^{124 a, 124 b}$, S. Roe ${ }^{30}$, O. Røhne ${ }^{119}$, S. Rolli ${ }^{161}$, A. Romaniouk ${ }^{98}$, M. Romano ${ }^{20 a, 20 b}$, S. M. Romano Saez ${ }^{34}$, E. Romero Adam ${ }^{167}$, N. Rompotis ${ }^{138}$, M. Ronzani ${ }^{48}$, L. Roos ${ }^{80}$, E. Ros ${ }^{167}$, S. Rosati ${ }^{132 a}$, K. Rosbach ${ }^{48}$, P. Rose ${ }^{137}$, P. L. Rosendahl ${ }^{14}$, O. Rosenthal ${ }^{141}$, V. Rossetti 146a,146b, E. Rossi104a,104b, L. P. Rossi ${ }^{50 a}, \quad$ R. Rosten ${ }^{138}, \quad$ M. Rotaru ${ }^{26 a}, \quad$ I. Roth ${ }^{172}$, J. Rothberg ${ }^{138}$, D. Rousseau ${ }^{117}$, C. R. Royon ${ }^{136}$, A. Rozanov ${ }^{85}$, Y. Rozen ${ }^{152}$, X. Ruan ${ }^{145 c}$, F. Rubbo ${ }^{143}$, I. Rubinskiy ${ }^{42}$, V. I. Rud ${ }^{99}$, C. Rudolph ${ }^{44}$, M. S. Rudolph ${ }^{158}$, F. Rühr ${ }^{48}$, A. Ruiz-Martinez ${ }^{30}$, Z. Rurikova ${ }^{48}$, N. A. Rusakovich ${ }^{65}$, A. Ruschke ${ }^{100}$, H. L. Russell ${ }^{138}$, J. P. Rutherfoord ${ }^{7}$, N. Ruthmann ${ }^{48}$, Y. F. Ryabov ${ }^{123}$, M. Rybar ${ }^{165}$, G. Rybkin ${ }^{117}$, N. C. Ryder $^{120}$, A. F. Saavedra ${ }^{150}$, G. Sabato ${ }^{107}$, S. Sacerdoti ${ }^{27}$, A. Saddique ${ }^{3}$, H. F-W. Sadrozinski ${ }^{137}$, R. Sadykov ${ }^{65}$, F. Safai Tehrani ${ }^{132 a}$, M. Saimpert ${ }^{136}$, T. Saito ${ }^{155}$, H. Sakamoto ${ }^{155}$, Y. Sakurai ${ }^{171}$, G. Salamanna ${ }^{134 a, 134 b}$, A. Salamon ${ }^{133 a}$, M. Saleem ${ }^{113}$, D. Salek ${ }^{107}$, P. H. Sales De Bruin ${ }^{138}$, D. Salihagic ${ }^{101}$, A. Salnikov ${ }^{143}$, J. Salt ${ }^{167}$, D. Salvatore ${ }^{37 a, 37 b}$, F. Salvatore ${ }^{149}$, A. Salvucci ${ }^{106}$, A. Salzburger ${ }^{30}$, D. Sammel ${ }^{48}$, D. Sampsonidis ${ }^{154}$, A. Sanchez ${ }^{104 a, 104 b}$, J. Sánchez ${ }^{167}$, $\begin{array}{lll}\text { V. Sanchez Martinez } & 167\end{array}$ H. Sandaker ${ }^{119}$, R. L. Sandbach ${ }^{76}, \quad$ H. G. Sander ${ }^{83}, \quad$ M. P. Sanders ${ }^{100,}$ M. Sandhoff ${ }^{175}$, C. Sandoval ${ }^{162}$, R. Sandstroem ${ }^{101}$, D. P. C. Sankey ${ }^{131}$, M. Sannino ${ }^{50 a, 50 b}$, A. Sansoni ${ }^{47}$, C. Santoni ${ }^{34}$, R. Santonico ${ }^{133 a, 133 b \text {, }}$ H. Santos ${ }^{126 a}$, I. Santoyo Castillo ${ }^{149}$, K. Sapp ${ }^{125}$, A. Sapronov ${ }^{65}$, J. G. Saraiva ${ }^{126 a, 126 d, ~ B . ~ S a r r a z i n ~}{ }^{21}$, O. Sasaki ${ }^{66}$, Y. Sasaki ${ }^{155}$, K. Sato ${ }^{160}$, G. Sauvage ${ }^{5, *}$, E. Sauvan ${ }^{5}$, G. Savage ${ }^{77}$, P. Savard ${ }^{158, d}$, C. Sawyer ${ }^{131}$, L. Sawyer ${ }^{79, n}$, J. Saxon ${ }^{31}$, C. Sbarra ${ }^{20 a}$, A. Sbrizzi ${ }^{20 a, 20 b}$, T. Scanlon ${ }^{78}$, D. A. Scannicchio ${ }^{163}$, M. Scarcella ${ }^{150}$, V. Scarfone ${ }^{37 a, 37 b}$, J. Schaarschmidt ${ }^{172}$, P. Schacht ${ }^{101}$, D. Schaefer ${ }^{30}$, R. Schaefer ${ }^{42}$, J. Schaeffer ${ }^{83}$, S. Schaepe ${ }^{21}$, S. Schaetzel ${ }^{58 b}$, U. Schäfer $^{83}$, A. C. Schaffer ${ }^{117}$, D. Schaile ${ }^{100}$, R. D. Schamberger ${ }^{148}$, V. Scharf ${ }^{58 a}$, V. A. Schegelsky ${ }^{123}$, D. Scheirich ${ }^{129}$, M. Schernau $^{163}$,

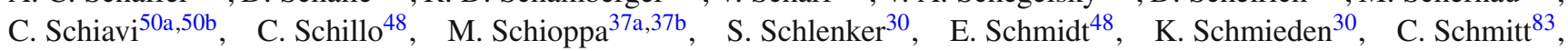
S. Schmitt ${ }^{58 b}$ ，S. Schmitt ${ }^{42}$ ， B. Schneider ${ }^{159 a}$ ，Y. J. Schnellbach ${ }^{74}$ ，U. Schnoor ${ }^{44}$, L. Schoeffel ${ }^{136}$, A. Schoening ${ }^{58 b}$, B. D. Schoenrock ${ }^{90}$, E. Schopf ${ }^{21}$, A. L. S. Schorlemmer ${ }^{54}$, M. Schott ${ }^{83}$, D. Schouten ${ }^{159 a}$, J. Schovancova ${ }^{8}$, S. Schramm ${ }^{49}$, M. Schreyer ${ }^{174}$, C. Schroeder ${ }^{83}$, N. Schuh ${ }^{83}$, M. J. Schultens ${ }^{21}$, H.-C. Schultz-Coulon ${ }^{58 a}$, H. Schulz ${ }^{16}$, M. Schumacher ${ }^{48}$,

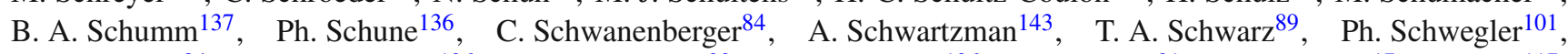
H. Schweiger ${ }^{84}$, Ph. Schwemling ${ }^{136}$, R. Schwienhorst ${ }^{90}$, J. Schwindling ${ }^{136}$, T. Schwindt ${ }^{21}$, F. G. Sciacca ${ }^{17}$, E. Scifo ${ }^{117}$, G. Sciolla ${ }^{23}$, F. Scuri ${ }^{124 a, 124 b}$, F. Scutti ${ }^{21}$, J. Searcy ${ }^{89}$, G. Sedov ${ }^{42}$, E. Sedykh ${ }^{123}$, P. Seema ${ }^{21}, \quad$ S. C. Seidel ${ }^{105}$, A. Seiden ${ }^{137}$, F. Seifert ${ }^{128}$, J. M. Seixas ${ }^{24 a}$, G. Sekhniaidze ${ }^{104 a}$, K. Sekhon ${ }^{89}$, S. J. Sekula ${ }^{40}$, D. M. Seliverstov ${ }^{123, *}$, N. Semprini-Cesari ${ }^{20 a}$,20b , C. Serfon ${ }^{30}$, L. Serin ${ }^{117}$, L. Serkin ${ }^{164 a, 164 b}$, T. Serre ${ }^{85}$, M. Sessa ${ }^{134 a, 134 b}$, R. Seuster ${ }^{159 a}$, H. Severini ${ }^{113}$, T. Sfiligoj ${ }^{75}$, F. Sforza ${ }^{30}$, A. Sfyrla ${ }^{30}$, E. Shabalina ${ }^{54}$, M. Shamim ${ }^{116}$, L. Y. Shan ${ }^{33 a}$, R. Shang ${ }^{165}$, J. T. Shank ${ }^{22}$, M. Shapiro ${ }^{15}$, P. B. Shatalov ${ }^{97}$, K. Shaw ${ }^{164 a, 164 b}$, S. M. Shaw ${ }^{84}$, A. Shcherbakova ${ }^{146 a, 146 b}$, C. Y. Shehu ${ }^{149}$, P. Sherwood $^{78}$, L. Shi ${ }^{151, \text { af }}$, S. Shimizu ${ }^{67}$, C. O. Shimmin ${ }^{163}$, M. Shimojima ${ }^{102}$, M. Shiyakova ${ }^{65}$, A. Shmeleva ${ }^{96}$, D. Shoaleh Saadi ${ }^{95}$, M. J. Shochet ${ }^{31}$, S. Shojaii ${ }^{91 a, 91 b}$, S. Shrestha ${ }^{111}$, E. Shulga ${ }^{98}$, M. A. Shupe ${ }^{7}$, S. Shushkevich ${ }^{42}$, P. Sicho ${ }^{127}$, P. E. Sidebo ${ }^{147}$, O. Sidiropoulou ${ }^{174}$, D. Sidorov ${ }^{114}$, A. Sidoti20a,20b ${ }^{20 . S^{2}}{ }^{2}{ }^{44}$, Dj. Sijacki ${ }^{13}$, J. Silva ${ }^{126 a, 126 d,}$ 
Y. Silver ${ }^{153}$ ， S. B. Silverstein ${ }^{146 a}$ ，V. Simak ${ }^{128}$ ，O. Simard ${ }^{5}$ ， Lj. Simic ${ }^{13}$ ，S. Simion ${ }^{117}$ ， E. Simioni ${ }^{83}$ ， B. Simmons ${ }^{78,}$ D. Simon ${ }^{34}$ ， R. Simoniello ${ }^{91 a, 91 b}$ ，P. Sinervo ${ }^{158}$ ，N. B. Sinev ${ }^{116}$ ，M. Sioli ${ }^{20 a, 20 b}$ ，G. Siragusa ${ }^{174}$ ， A. N. Sisakyan ${ }^{65, *}$, S. Yu. Sivoklokov ${ }^{99}$ ，J. Sjölin 146a,146b， T. B. Sjursen ${ }^{14}$ ， M. B. Skinner ${ }^{72}$ ，H. P. Skottowe ${ }^{57}$ ，P. Skubic ${ }^{113}$ ， M. Slater ${ }^{18}$, T. Slavicek ${ }^{128}$, M. Slawinska ${ }^{107}$, K. Sliwa ${ }^{161}$, V. Smakhtin ${ }^{172}$, B. H. Smart ${ }^{46}$, L. Smestad ${ }^{14}$, S. Yu. Smirnov ${ }^{98}$, Y. Smirnov ${ }^{98}$, L. N. Smirnova ${ }^{99, a g}$, O. Smirnova ${ }^{81}$, M. N. K. Smith ${ }^{35}$, R. W. Smith ${ }^{35}$, M. Smizanska ${ }^{72}$, K. Smolek ${ }^{128}$, A. A. Snesarev ${ }^{96}$, G. Snidero ${ }^{76}$, S. Snyder ${ }^{25}$ ， R. Sobie ${ }^{169, k}$, F. Socher ${ }^{44}$, A. Soffer ${ }^{153}$, D. A. Soh ${ }^{151, \text { af }}$, C. A. Solans ${ }^{30}$, M. Solar ${ }^{128}$, J. Solc ${ }^{128}$, E. Yu. Soldatov ${ }^{98}$, U. Soldevila ${ }^{167}$, A. A. Solodkov ${ }^{130}$, A. Soloshenko ${ }^{65}$, O. V. Solovyanov ${ }^{130}$, V. Solovyev ${ }^{123}$, P. Sommer ${ }^{48}$, H. Y. Song ${ }^{33 b}$, N. Soni ${ }^{1}$, A. Sood ${ }^{15}$, A. Sopczak ${ }^{128}$, B. Sopko ${ }^{128}$, V. Sopko ${ }^{128}$, V. Sorin ${ }^{12}$, D. Sosa ${ }^{58 b}$, M. Sosebee ${ }^{8}$, C. L. Sotiropoulou124a,124b, R. Soualah ${ }^{164 a, 164 c}$, A. M. Soukharev109,c, D. South ${ }^{42}$ ， B. C. Sowden ${ }^{77}$, S. Spagnolo ${ }^{73 a, 73 b}$, M. Spalla ${ }^{124 a, 124 b}$, F. Spanò ${ }^{77}$, W. R. Spearman ${ }^{57}$, D. Sperlich ${ }^{16}$, F. Spettel ${ }^{101}$, R. Spighi ${ }^{20 a}$, G. Spigo $^{30}$, L. A. Spiller ${ }^{88}$, M. Spousta ${ }^{129}$, T. Spreitzer ${ }^{158}$, R. D. St. Denis ${ }^{53, *}$, S. Staerz $^{44}$, J. Stahlman ${ }^{122}$, R. Stamen ${ }^{58 a}$, S. Stamm ${ }^{16}$, E. Stanecka ${ }^{39}$, C. Stanescu ${ }^{134 a}$, M. Stanescu-Bellu ${ }^{42}$, M. M. Stanitzki ${ }^{42}$, S. Stapnes ${ }^{119}$, E. A. Starchenko ${ }^{130}$, J. Stark ${ }^{55}$, P. Staroba ${ }^{127}$, P. Starovoitov ${ }^{42}$, R. Staszewski ${ }^{39}$, P. Stavinaa ${ }^{144 a, *}$, P. Steinberg ${ }^{25}$, B. Stelzer ${ }^{142}$, H. J. Stelzer ${ }^{30}$, O. Stelzer-Chilton ${ }^{159 a}$, H. Stenzel ${ }^{52}$, G. A. Stewart ${ }^{53}$ ，J. A. Stillings ${ }^{21}$ ，M. C. Stockton ${ }^{87}$ ，M. Stoebe ${ }^{87}$ ， G. Stoicea ${ }^{26 a}$, P. Stolte ${ }^{54}$, S. Stonjek ${ }^{101}$, A. R. Stradling ${ }^{8}$, A. Straessner ${ }^{44}$, M. E. Stramaglia ${ }^{17}$, J. Strandberg ${ }^{147}$, S. Strandberg ${ }^{146 a, 146 b}$, A. Strandlie ${ }^{119}$ ， E. Strauss ${ }^{143}$ ， M. Strauss ${ }^{113}$ ，P. Strizenec ${ }^{144 b}$ ， R. Ströhmer ${ }^{174}$ ， D. M. Strom ${ }^{116}$ ， R. Stroynowski ${ }^{40}$, A. Strubig ${ }^{106}$, S. A. Stucci ${ }^{17}$, B. Stugu ${ }^{14}$, N. A. Styles ${ }^{42}$, D. $\mathrm{Su}^{143}$, J. Su ${ }^{125}$, R. Subramaniam ${ }^{79}$, A. Succurro ${ }^{12}$, Y. Sugaya ${ }^{118}$, C. Suhr ${ }^{108}$, M. Suk ${ }^{128}$, V. V. Sulin ${ }^{96}$, S. Sultansoy ${ }^{4 c}$, T. Sumida ${ }^{68}$, S. Sun ${ }^{57}$, X. Sun ${ }^{33 a}$, J. E. Sundermann ${ }^{48}$, K. Suruliz ${ }^{149}$, G. Susinno ${ }^{37 a, 37 b}$, M. R. Sutton ${ }^{149}$, S. Suzuki ${ }^{66}$, M. Svatos ${ }^{127}$, S. Swedish ${ }^{168}$, M. Swiatlowski ${ }^{143}$, I. Sykora ${ }^{144 a}$, T. Sykora ${ }^{129}$, D. Ta ${ }^{90}$, C. Taccini ${ }^{134 a}{ }^{134 b}$, K. Tackmann ${ }^{42}$, J. Taenzer ${ }^{158}$, A. Taffard ${ }^{163}$, R. Tafirout ${ }^{159 a}$, N. Taiblum ${ }^{153}$, H. Takai ${ }^{25}$, R. Takashima ${ }^{69}$, H. Takeda ${ }^{67}$, T. Takeshita ${ }^{140}$, Y. Takubo ${ }^{66}$, M. Talby ${ }^{85}$, A. A. Talyshev ${ }^{109, c}$, J. Y. C. Tam ${ }^{174}$, K. G. Tan $^{88}$, J. Tanaka ${ }^{155}$, R. Tanaka ${ }^{117}$, S. Tanaka ${ }^{66}$, B. B. Tannenwald ${ }^{111}$, N. Tannoury ${ }^{21}$, S. Tapprogge ${ }^{83}$, S. Tarem ${ }^{152}$, F. Tarrade ${ }^{29}$, G. F. Tartarelli ${ }^{91 a}$, P. Tas ${ }^{129}$, M. Tasevsky ${ }^{127}$, T. Tashiro ${ }^{68}$, E. Tassi ${ }^{37 a, 37 b}$, A. Tavares Delgado ${ }^{126 a, 126 b}$, Y. Tayalati ${ }^{135 \mathrm{~d}}$, F. E. Taylor ${ }^{94}$, G. N. Taylor ${ }^{88}$, W. Taylor ${ }^{159 b}$, F. A. Teischinger ${ }^{30}$, M. Teixeira Dias Castanheira ${ }^{76}$, P. Teixeira-Dias ${ }^{77}$, K. K. Temming ${ }^{48}$, H. Ten Kate ${ }^{30}$, P. K. Teng ${ }^{151}$, J. J. Teoh ${ }^{118}$, F. Tepel ${ }^{175}$, S. Terada ${ }^{66}$, K. Terashi ${ }^{155}$, J. Terron ${ }^{82}$, S. Terzo ${ }^{101}$, M. Testa ${ }^{47}$, R. J. Teuscher ${ }^{158, \mathrm{k}}$, T. Theveneaux-Pelzer ${ }^{34}$, J. P. Thomas ${ }^{18}$, J. Thomas-Wilsker ${ }^{77}$, E. N. Thompson ${ }^{35}$, P. D. Thompson ${ }^{18}$, R. J. Thompson ${ }^{84}$, A. S. Thompson ${ }^{53}$, L. A. Thomsen ${ }^{176}$, E. Thomson ${ }^{122}$,

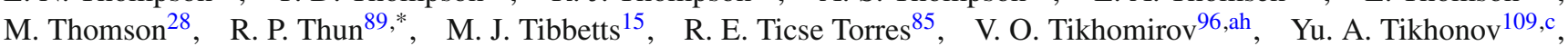
S. Timoshenko ${ }^{98}$, E. Tiouchichine ${ }^{85}$, P. Tipton ${ }^{176}$, S. Tisserant ${ }^{85}$, K. Todome ${ }^{157}$, T. Todorov ${ }^{5, *}$, S. Todorova-Nova ${ }^{129}$, J. Tojo ${ }^{70}$, S. Tokár ${ }^{144 a}$, K. Tokushuku ${ }^{66}$, K. Tollefson ${ }^{90}$, E. Tolley ${ }^{57}$, L. Tomlinson ${ }^{84}$, M. Tomoto ${ }^{103}$, L. Tompkins ${ }^{143, \text { ai }}$, K. Toms ${ }^{105}$, E. Torrence ${ }^{116}$, H. Torres ${ }^{142}$, E. Torró Pastor ${ }^{167}$, J. Toth ${ }^{85}$,aj, F. Touchard ${ }^{85}$, D. R. Tovey ${ }^{139}$, T. Trefzger ${ }^{174}$, L. Tremblet ${ }^{30}$, A. Tricoli $^{30}$, I. M. Trigger ${ }^{159 a}$, S. Trincaz-Duvoid ${ }^{80}$, M. F. Tripiana ${ }^{12}$, W. Trischuk ${ }^{158}$, B. Trocmé ${ }^{55}$, C. Troncon ${ }^{91 a}$, M. Trottier-McDonald ${ }^{15}$, M. Trovatelli ${ }^{169}$, P. True ${ }^{90}$, L. Truong ${ }^{164 a, 164 c}$, M. Trzebinski ${ }^{39}$, A. Trzupek ${ }^{39}$, C. Tsarouchas ${ }^{30}$, J. C-L. Tseng ${ }^{120}$, P. V. Tsiareshka ${ }^{92}$, D. Tsionou ${ }^{154}$, G. Tsipolitis ${ }^{10}$, N. Tsirintanis ${ }^{9}$, S. Tsiskaridze ${ }^{12}$, V. Tsiskaridze ${ }^{48}$, E. G. Tskhadadze ${ }^{51 a}$, I. I. Tsukerman ${ }^{97}$, V. Tsulaia ${ }^{15}$, S. Tsuno ${ }^{66}$, D. Tsybychev ${ }^{148}$, A. Tudorache ${ }^{26 a}$, V. Tudorache ${ }^{26 a}$, A. N. Tuna ${ }^{122}$, S. A. Tupputi $20 \mathrm{a}, 20 \mathrm{~b}$, S. Turchikhin ${ }^{99, a g}$, D. Turecek ${ }^{128}$, R. Turra ${ }^{91 a, 91 b}$, A. J. Turvey ${ }^{40}$,

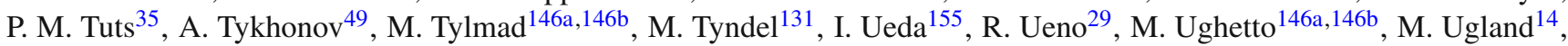
M. Uhlenbrock ${ }^{21}$, F. Ukegawa ${ }^{160}$, G. Unal ${ }^{30}$, A. Undrus ${ }^{25}$, G. Unel ${ }^{163}$, F. C. Ungaro ${ }^{48}$, Y. Unno ${ }^{66}$, C. Unverdorben ${ }^{100}$, J. Urban ${ }^{144 b}$, P. Urquijo ${ }^{88}$, P. Urrejola ${ }^{83}$, G. Usai ${ }^{8}$, A. Usanova ${ }^{62}$, L. Vacavant ${ }^{85}$, V. Vacek ${ }^{128}$, B. Vachon ${ }^{87}$, C. Valderanis ${ }^{83}$, N. Valencic ${ }^{107}$, S. Valentinetti ${ }^{20 a, 20 b}$, A. Valero ${ }^{167}$, L. Valery ${ }^{12}$, S. Valkar ${ }^{129}$, E. Valladolid Gallego ${ }^{167}$, S. Vallecorsa ${ }^{49}$, $\begin{array}{ll}\text { J. A. Valls Ferrer } & 167\end{array}$ W. Van Den Wollenberg ${ }^{107}$, P. C. Van Der Deijl ${ }^{107}$, R. van der Geer ${ }^{107}$, H. van der Graaf ${ }^{107}$, R. Van Der Leeuw ${ }^{107}$, N. van Eldik $^{152}$, P. van Gemmeren ${ }^{6}$, J. Van Nieuwkoop ${ }^{142}$, I. van Vulpen ${ }^{107}$, M. C. van Woerden ${ }^{30}$, M. Vanadia ${ }^{132 a, 132 b}$, W. Vandelli ${ }^{30}$, R. Vanguri ${ }^{122}$, A. Vaniachine ${ }^{6}, \quad$ F. Vannucci ${ }^{80}$, G. Vardanyan ${ }^{177}$, R. Vari ${ }^{132 a}$, E. W. Varnes ${ }^{7}$, T. Varol ${ }^{40}$, D. Varouchas ${ }^{80}$, A. Vartapetian ${ }^{8}$, K. E. Varvell ${ }^{150}$, F. Vazeille ${ }^{34}$, T. Vazquez Schroeder ${ }^{87}$, J. Veatch ${ }^{7}$, L. M. Veloce ${ }^{158}$, F. Veloso ${ }^{126 a}, 126 c$, T. Velz ${ }^{21}$, S. Veneziano ${ }^{132 a}$, A. Ventura ${ }^{73 a, 73 b}$, D. Ventura ${ }^{86}$, M. Venturi ${ }^{169}$, N. Venturi ${ }^{158}$, A. Venturini ${ }^{23}$, V. Vercesi ${ }^{121 a}$, M. Verducci ${ }^{132 a, 132 b}$, W. Verkerke ${ }^{107}$, J. C. Vermeulen ${ }^{107}$, A. Vest ${ }^{44}$, M. C. Vetterli ${ }^{142, d}$, O. Viazlo ${ }^{81}$, I. Vichou ${ }^{165}$, T. Vickey ${ }^{139}$, O. E. Vickey Boeriu ${ }^{139}$, G. H. A. Viehhauser ${ }^{120}$, S. Viel ${ }^{15}$, R. Vigne ${ }^{62}$, M. Villa ${ }^{20 a, 20 b}$, M. Villaplana Perez ${ }^{11 a, 91 b}$, E. Vilucchi ${ }^{47}$, M. G. Vincter ${ }^{29}$, V. B. Vinogradov ${ }^{65}$, I. Vivarelli ${ }^{149}$, F. Vives Vaque ${ }^{3}$, S. Vlachos ${ }^{10}$, D. Vladoiu ${ }^{100}$, M. Vlasak ${ }^{128}$, M. Vogel ${ }^{32 a}$, P. Vokac ${ }^{128}$, G. Volpi ${ }^{124 a, 124 b}$, M. Volpi ${ }^{88}$, H. von der Schmitt ${ }^{101}$, H. von Radziewski ${ }^{48}$, E. von Toerne ${ }^{21}$, V. Vorobel ${ }^{129}$ ，K. Vorobev ${ }^{98}$, M. Vos ${ }^{167}$, R. Voss ${ }^{30}$, J. H. Vossebeld ${ }^{74}$, N. Vranjes ${ }^{13}$, M. Vranjes Milosavljevic ${ }^{13}$, V. Vrba ${ }^{127}$, M. Vreeswijk ${ }^{107}$, R. Vuillermet ${ }^{30}$, I. Vukotic ${ }^{31}$, Z. Vykydal ${ }^{128}$, P. Wagner ${ }^{21}$, W. Wagner ${ }^{175}$, H. Wahlberg ${ }^{71}$, S. Wahrmund ${ }^{44}$, J. Wakabayashi ${ }^{103}$, J. Walder ${ }^{72}$, R. Walker ${ }^{100}$, W. Walkowiak ${ }^{141}$, C. Wang ${ }^{151}$, F. Wang ${ }^{173}$, H. Wang ${ }^{15}$, H. Wang ${ }^{40}$, J. Wang ${ }^{42}$, J. Wang ${ }^{33 a}$, K. Wang ${ }^{87}$, R. Wang ${ }^{6}$, 
S. M. Wang ${ }^{151}$, T. Wang ${ }^{21}$, T. Wang ${ }^{35}$, X. Wang ${ }^{176}$, C. Wanotayaroj ${ }^{116}$, A. Warburton ${ }^{87}$, C. P. Ward ${ }^{28}$, D. R. Wardrope ${ }^{78}$, M. Warsinsky ${ }^{48}$, A. Washbrook ${ }^{46}$, C. Wasicki ${ }^{42}$, P. M. Watkins ${ }^{18}$, A. T. Watson ${ }^{18}$, I. J. Watson ${ }^{150}$, M. F. Watson ${ }^{18}$, G. Watts ${ }^{138}$, S. Watts ${ }^{84}$, B. M. Waugh ${ }^{78}$, S. Webb ${ }^{84}$, M. S. Weber ${ }^{17}$, S. W. Weber ${ }^{174}$, J. S. Webster ${ }^{31}$, A. R. Weidberg ${ }^{120}$, B. Weinert ${ }^{61}$, J. Weingarten ${ }^{54}$, C. Weiser ${ }^{48}$, H. Weits ${ }^{107}$, P. S. Wells ${ }^{30}$, T. Wenaus ${ }^{25}$, T. Wengler ${ }^{30}$, S. Wenig ${ }^{30}$, N. Wermes ${ }^{21}$, M. Werner ${ }^{48}$, P. Werner ${ }^{30}$, M. Wessels ${ }^{58 a}$, J. Wetter ${ }^{161}$, K. Whalen ${ }^{116}$, A. M. Wharton ${ }^{72}$, A. White ${ }^{8}$, M. J. White ${ }^{1}$, R. White ${ }^{32 b}$, S. White ${ }^{124 a, 124 b}$, D. Whiteson ${ }^{163}$, F. J. Wickens ${ }^{131}$, W. Wiedenmann ${ }^{173}$, M. Wielers ${ }^{131}$, P. Wienemann ${ }^{21}$, C. Wiglesworth ${ }^{36}$, L. A. M. Wiik-Fuchs ${ }^{21}$, A. Wildauer ${ }^{101}$, H. G. Wilkens ${ }^{30}$, H. H. Williams ${ }^{122}$, S. Williams ${ }^{107}$, C. Willis $^{90}$, S. Willocq ${ }^{86}$, A. Wilson ${ }^{89}$, J. A. Wilson ${ }^{18}$, I. Wingerter-Seez ${ }^{5}$, F. Winklmeier ${ }^{116}$, B. T. Winter ${ }^{21}$, M. Wittgen ${ }^{143}$, J. Wittkowski ${ }^{100}$, S. J. Wollstadt ${ }^{83}$, M. W. Wolter ${ }^{39}$, H. Wolters ${ }^{126 a, 126 c}$, B. K. Wosiek ${ }^{39}$, J. Wotschack ${ }^{30}$, M. J. Woudstra ${ }^{84}$, K. W. Wozniak ${ }^{39}$, M. Wu ${ }^{55}$, M. Wu ${ }^{31}$, S. L. Wu ${ }^{173}$, X. Wu ${ }^{49}$, Y. Wu ${ }^{89}$, T. R. Wyatt ${ }^{84}$, B. M. Wynne ${ }^{46}$, S. Xella ${ }^{36}$, D. Xu ${ }^{33 a}$, L. Xu ${ }^{33 b, a k}$, B. Yabsley ${ }^{150}$, S. Yacoob ${ }^{145 a}$, R. Yakabe ${ }^{67}$, M. Yamada ${ }^{66}$, Y. Yamaguchi ${ }^{118}$, A. Yamamoto ${ }^{66}$, S. Yamamoto ${ }^{155}$, T. Yamanaka ${ }^{155}$, K. Yamauchi ${ }^{103}$, Y. Yamazaki ${ }^{67}$, Z. Yan ${ }^{22}$, H. Yang ${ }^{33 e}$, H. Yang ${ }^{173}$, Y. Yang ${ }^{151}$, W-M. Yao ${ }^{15}$, Y. Yasu ${ }^{66}$, E. Yatsenko ${ }^{5}$, K. H. Yau Wong ${ }^{21}$, J. Ye ${ }^{40}$, S. Ye ${ }^{25}$, I. Yeletskikh ${ }^{65}$, A. L. Yen ${ }^{57}$, E. Yildirim ${ }^{42}$, K. Yorita ${ }^{171}$, R. Yoshida ${ }^{6}$, K. Yoshihara ${ }^{122}$, C. Young ${ }^{143}$, C. J. S. Young ${ }^{30}$, S. Youssef ${ }^{22}$, D. R. Yu ${ }^{15}$, J. Yu ${ }^{8}$, J. M. Yu ${ }^{89}$, J. Yu $u^{114}$, L. Yuan ${ }^{67}$, S. P. Y. Yuen ${ }^{21}$, A. Yurkewicz ${ }^{108}$, I. Yusuff ${ }^{28, a l}$, B. Zabinski ${ }^{39}$, R. Zaidan ${ }^{63}$, A. M. Zaitsev ${ }^{130, a b}$, J. Zalieckas ${ }^{14}$, A. Zaman ${ }^{148}$, S. Zambito ${ }^{57}$, L. Zanello ${ }^{132 a, 132 b}$, D. Zanzi ${ }^{88}$, C. Zeitnitz ${ }^{175}$, M. Zeman ${ }^{128}$, A. Zemla ${ }^{38 a}$, K. Zengel ${ }^{23}$, O. Zenin ${ }^{130}$, T. Ženiš ${ }^{144 a}$, D. Zerwas ${ }^{117}$, D. Zhang ${ }^{89}$, F. Zhang ${ }^{173}$, H. Zhang ${ }^{33 c}$, J. Zhang ${ }^{6}$, L. Zhang ${ }^{48}$, R. Zhang ${ }^{33 b}$, X. Zhang ${ }^{33 d}$, Z. Zhang ${ }^{117}$, X. Zhao ${ }^{40}$, Y. Zhao ${ }^{33 d, 117}$, Z. Zhao ${ }^{33 b}$, A. Zhemchugov ${ }^{65}$, J. Zhong ${ }^{120}$, B. Zhou ${ }^{89}$, C. Zhou ${ }^{45}$, L. Zhou ${ }^{35}$, L. Zhou ${ }^{40}$, N. Zhou ${ }^{163}$, C. G. Zhu ${ }^{33 d}$, H. Zhu ${ }^{33 a}$, J. Zhu ${ }^{89}$, Y. Zhu ${ }^{33 b}$, X. Zhuang ${ }^{33 a}$, K. Zhukov ${ }^{96}$, A. Zibell ${ }^{174}$, D. Zieminska ${ }^{61}$, N. I. Zimine ${ }^{65}$, C. Zimmermann ${ }^{83}$, S.Zimmermann ${ }^{48}$, Z. Zinonos ${ }^{54}$, M. Zinser ${ }^{83}$, M. Ziolkowski ${ }^{141}$, L. Živković ${ }^{13}$, G. Zobernig ${ }^{173}$, A. Zoccoli ${ }^{20 a, 20 b}$, M. zur Nedden ${ }^{16}$, G. Zurzolo ${ }^{104 a, 104 b}$, L. Zwalinski $^{30}$

${ }^{1}$ Department of Physics, University of Adelaide, Adelaide, Australia

${ }^{2}$ Physics Department, SUNY Albany, Albany, NY, USA

${ }^{3}$ Department of Physics, University of Alberta, Edmonton, AB, Canada

4 (a) Department of Physics, Ankara University, Ankara, Turkey; ${ }^{(b)}$ Istanbul Aydin University, Istanbul,

Turkey; ${ }^{(c)}$ Division of Physics, TOBB University of Economics and Technology, Ankara, Turkey

${ }^{5}$ LAPP, CNRS/IN2P3 and Université Savoie Mont Blanc, Annecy-le-Vieux, France

${ }^{6}$ High Energy Physics Division, Argonne National Laboratory, Argonne, IL, USA

${ }^{7}$ Department of Physics, University of Arizona, Tucson, AZ, USA

${ }^{8}$ Department of Physics, The University of Texas at Arlington, Arlington, TX, USA

${ }^{9}$ Physics Department, University of Athens, Athens, Greece

${ }^{10}$ Physics Department, National Technical University of Athens, Zografou, Greece

${ }^{11}$ Institute of Physics, Azerbaijan Academy of Sciences, Baku, Azerbaijan

12 Institut de Física d'Altes Energies and Departament de Física de la Universitat Autònoma de Barcelona, Barcelona, Spain

${ }^{13}$ Institute of Physics, University of Belgrade, Belgrade, Serbia

${ }^{14}$ Department for Physics and Technology, University of Bergen, Bergen, Norway

${ }^{15}$ Physics Division, Lawrence Berkeley National Laboratory and University of California, Berkeley, CA, USA

${ }^{16}$ Department of Physics, Humboldt University, Berlin, Germany

${ }^{17}$ Albert Einstein Center for Fundamental Physics and Laboratory for High Energy Physics, University of Bern, Bern, Switzerland

${ }^{18}$ School of Physics and Astronomy, University of Birmingham, Birmingham, UK

19 (a) Department of Physics, Bogazici University, Istanbul, Turkey; ${ }^{(b)}$ Department of Physics Engineering, Gaziantep University, Gaziantep, Turkey; ${ }^{(c)}$ Department of Physics, Dogus University, Istanbul, Turkey

20 (a) INFN Sezione di Bologna, Bologna, Italy; ${ }^{\left({ }^{b}\right)}$ Dipartimento di Fisica e Astronomia, Università di Bologna, Bologna, Italy

${ }^{21}$ Physikalisches Institut, University of Bonn, Bonn, Germany

22 Department of Physics, Boston University, Boston, MA, USA

${ }^{23}$ Department of Physics, Brandeis University, Waltham, MA, USA

24 (a) Universidade Federal do Rio De Janeiro COPPE/EE/IF, Rio de Janeiro, Brazil; ${ }^{(b)}$ Electrical Circuits Department, Federal University of Juiz de Fora (UFJF), Juiz de Fora, Brazil; ${ }^{(c)}$ Federal University of Sao Joao del Rei (UFSJ), Sao Joao del Rei, Brazil; ${ }^{(d)}$ Instituto de Fisica, Universidade de Sao Paulo, São Paulo, Brazil

${ }^{25}$ Physics Department, Brookhaven National Laboratory, Upton, NY, USA 
26 (a) National Institute of Physics and Nuclear Engineering, Bucharest, Romania; ${ }^{(b)}$ Physics Department, National Institute for Research and Development of Isotopic and Molecular Technologies, Cluj Napoca, Romania; ${ }^{\left({ }^{c}\right)}$ University Politehnica Bucharest, Bucharest, Romania; ${ }^{(d)}$ West University in Timisoara, Timisoara, Romania

${ }^{27}$ Departamento de Física, Universidad de Buenos Aires, Buenos Aires, Argentina

${ }^{28}$ Cavendish Laboratory, University of Cambridge, Cambridge, UK

${ }^{29}$ Department of Physics, Carleton University, Ottawa, ON, Canada

${ }^{30}$ CERN, Geneva, Switzerland

${ }^{31}$ Enrico Fermi Institute, University of Chicago, Chicago, IL, USA

32 (a) Departamento de Física, Pontificia Universidad Católica de Chile, Santiago, Chile; ${ }^{(b)}$ Departamento de Física, Universidad Técnica Federico Santa María, Valparaiso, Chile

33 (a) Institute of High Energy Physics, Chinese Academy of Sciences, Beijing, China; ${ }^{(b)}$ Department of Modern Physics, University of Science and Technology of China, Hefei, Anhui, China; ${ }^{(c)}$ Department of Physics, Nanjing University, Nanjing, Jiangsu, China; ${ }^{(d)}$ School of Physics, Shandong University, Shandong, China; ${ }^{(e)}$ Shanghai Key Laboratory for Particle Physics and Cosmology, Department of Physics and Astronomy, Shanghai Jiao Tong University, Shanghai, China; ${ }^{(f)}$ Physics Department, Tsinghua University, Beijing 100084, China

${ }^{34}$ Laboratoire de Physique Corpusculaire, Clermont Université and Université Blaise Pascal and CNRS/IN2P3, Clermont-Ferrand, France

${ }^{35}$ Nevis Laboratory, Columbia University, Irvington, NY, USA

${ }^{36}$ Niels Bohr Institute, University of Copenhagen, Copenhagen, Denmark

37 (a) INFN Gruppo Collegato di Cosenza, Laboratori Nazionali di Frascati, Frascati, Italy; ${ }^{(b)}$ Dipartimento di Fisica, Università della Calabria, Rende, Italy

38 (a) AGH University of Science and Technology, Faculty of Physics and Applied Computer Science, Kraków,

Poland; ${ }^{(b)}$ Marian Smoluchowski Institute of Physics, Jagiellonian University, Kraków, Poland

${ }^{39}$ Institute of Nuclear Physics, Polish Academy of Sciences, Kraków, Poland

${ }^{40}$ Physics Department, Southern Methodist University, Dallas, TX, USA

${ }^{41}$ Physics Department, University of Texas at Dallas, Richardson, TX, USA

42 DESY, Hamburg and Zeuthen, Germany

${ }^{43}$ Institut für Experimentelle Physik IV, Technische Universität Dortmund, Dortmund, Germany

${ }^{44}$ Institut für Kern- und Teilchenphysik, Technische Universität Dresden, Dresden, Germany

${ }^{45}$ Department of Physics, Duke University, Durham, NC, USA

${ }^{46}$ SUPA-School of Physics and Astronomy, University of Edinburgh, Edinburgh, UK

${ }^{47}$ INFN Laboratori Nazionali di Frascati, Frascati, Italy

${ }^{48}$ Fakultät für Mathematik und Physik, Albert-Ludwigs-Universität, Freiburg, Germany

${ }^{49}$ Section de Physique, Université de Genève, Geneva, Switzerland

50 (a) INFN Sezione di Genova, Genoa, Italy; ${ }^{(b)}$ Dipartimento di Fisica, Università di Genova, Genoa, Italy

51 (a) E. Andronikashvili Institute of Physics, Iv. Javakhishvili Tbilisi State University, Tbilisi, Georgia; ${ }^{(b)}$ High Energy

Physics Institute, Tbilisi State University, Tbilisi, Georgia

52 II Physikalisches Institut, Justus-Liebig-Universität Giessen, Giessen, Germany

${ }^{53}$ SUPA-School of Physics and Astronomy, University of Glasgow, Glasgow, UK

54 II Physikalisches Institut, Georg-August-Universität, Göttingen, Germany

${ }^{55}$ Laboratoire de Physique Subatomique et de Cosmologie, Université Grenoble-Alpes, CNRS/IN2P3, Grenoble, France

${ }^{56}$ Department of Physics, Hampton University, Hampton, VA, USA

${ }^{57}$ Laboratory for Particle Physics and Cosmology, Harvard University, Cambridge, MA, USA

58 (a) Kirchhoff-Institut für Physik, Ruprecht-Karls-Universität Heidelberg, Heidelberg, Germany; ${ }^{(b)}$ Physikalisches Institut, Ruprecht-Karls-Universität Heidelberg, Heidelberg, Germany; ${ }^{(c)}$ ZITI Institut für technische Informatik,

Ruprecht-Karls-Universität Heidelberg, Mannheim, Germany

${ }^{59}$ Faculty of Applied Information Science, Hiroshima Institute of Technology, Hiroshima, Japan

60 (a) Department of Physics, The Chinese University of Hong Kong, Shatin, NT, Hong Kong; ${ }^{\left({ }^{b}\right)}$ Department of Physics, The University of Hong Kong, Hong Kong, Hong Kong; ${ }^{(c)}$ Department of Physics, The Hong Kong University of Science and Technology, Clear Water Bay, Kowloon, Hong Kong, China

${ }^{61}$ Department of Physics, Indiana University, Bloomington, IN, USA

${ }^{62}$ Institut für Astro- und Teilchenphysik, Leopold-Franzens-Universität, Innsbruck, Austria

${ }^{63}$ University of Iowa, Iowa City, IA, USA 
${ }^{64}$ Department of Physics and Astronomy, Iowa State University, Ames, IA, USA

65 Joint Institute for Nuclear Research, JINR Dubna, Dubna, Russia

${ }^{66}$ KEK, High Energy Accelerator Research Organization, Tsukuba, Japan

${ }^{67}$ Graduate School of Science, Kobe University, Kobe, Japan

${ }^{68}$ Faculty of Science, Kyoto University, Kyoto, Japan

${ }^{69}$ Kyoto University of Education, Kyoto, Japan

${ }^{70}$ Department of Physics, Kyushu University, Fukuoka, Japan

${ }^{71}$ Instituto de Física La Plata, Universidad Nacional de La Plata and CONICET, La Plata, Argentina

72 Physics Department, Lancaster University, Lancaster, UK

73 (a) INFN Sezione di Lecce, Lecce, Italy; ${ }^{\text {(b) }}$ Dipartimento di Matematica e Fisica, Università del Salento, Lecce, Italy

${ }^{74}$ Oliver Lodge Laboratory, University of Liverpool, Liverpool, UK

${ }^{75}$ Department of Physics, Jožef Stefan Institute and University of Ljubljana, Ljubljana, Slovenia

76 School of Physics and Astronomy, Queen Mary University of London, London, UK

77 Department of Physics, Royal Holloway University of London, Surrey, UK

${ }^{78}$ Department of Physics and Astronomy, University College London, London, UK

${ }^{79}$ Louisiana Tech University, Ruston, LA, USA

${ }^{80}$ Laboratoire de Physique Nucléaire et de Hautes Energies, UPMC and Université Paris-Diderot and CNRS/IN2P3, Paris, France

${ }^{81}$ Fysiska institutionen, Lunds universitet, Lund, Sweden

82 Departamento de Fisica Teorica C-15, Universidad Autonoma de Madrid, Madrid, Spain

${ }^{83}$ Institut für Physik, Universität Mainz, Mainz, Germany

${ }^{84}$ School of Physics and Astronomy, University of Manchester, Manchester, UK

${ }^{85}$ CPPM, Aix-Marseille Université and CNRS/IN2P3, Marseille, France

${ }^{86}$ Department of Physics, University of Massachusetts, Amherst, MA, USA

${ }^{87}$ Department of Physics, McGill University, Montreal, QC, Canada

${ }^{88}$ School of Physics, University of Melbourne, Melbourne, VIC, Australia

${ }^{89}$ Department of Physics, The University of Michigan, Ann Arbor, MI, USA

${ }^{90}$ Department of Physics and Astronomy, Michigan State University, East Lansing, MI, USA

91 (a) INFN Sezione di Milano, Milan, Italy; ${ }^{(b)}$ Dipartimento di Fisica, Università di Milano, Milan, Italy

92 B.I. Stepanov Institute of Physics, National Academy of Sciences of Belarus, Minsk, Republic of Belarus

${ }^{93}$ National Scientific and Educational Centre for Particle and High Energy Physics, Minsk, Republic of Belarus

${ }^{94}$ Department of Physics, Massachusetts Institute of Technology, Cambridge, MA, USA

${ }^{95}$ Group of Particle Physics, University of Montreal, Montreal, QC, Canada

${ }^{96}$ P.N. Lebedev Institute of Physics, Academy of Sciences, Moscow, Russia

${ }^{97}$ Institute for Theoretical and Experimental Physics (ITEP), Moscow, Russia

${ }^{98}$ National Research Nuclear University MEPhI, Moscow, Russia

${ }^{99}$ D.V. Skobeltsyn Institute of Nuclear Physics, M.V. Lomonosov Moscow State University, Moscow, Russia

${ }^{100}$ Fakultät für Physik, Ludwig-Maximilians-Universität München, Munich, Germany

${ }^{101}$ Max-Planck-Institut für Physik (Werner-Heisenberg-Institut), Munich, Germany

${ }^{102}$ Nagasaki Institute of Applied Science, Nagasaki, Japan

${ }^{103}$ Graduate School of Science and Kobayashi-Maskawa Institute, Nagoya University, Nagoya, Japan

104 (a) INFN Sezione di Napoli, Naples, Italy; ${ }^{(b)}$ Dipartimento di Fisica, Università di Napoli, Naples, Italy

105 Department of Physics and Astronomy, University of New Mexico, Albuquerque, NM, USA

${ }^{106}$ Institute for Mathematics, Astrophysics and Particle Physics, Radboud University Nijmegen/Nikhef, Nijmegen, The Netherlands

${ }^{107}$ Nikhef National Institute for Subatomic Physics and University of Amsterdam, Amsterdam, The Netherlands

${ }^{108}$ Department of Physics, Northern Illinois University, De Kalb, IL, USA

${ }^{109}$ Budker Institute of Nuclear Physics, SB RAS, Novosibirsk, Russia

${ }^{110}$ Department of Physics, New York University, New York, NY, USA

111 Ohio State University, Columbus, OH, USA

${ }^{112}$ Faculty of Science, Okayama University, Okayama, Japan

${ }^{113}$ Homer L. Dodge Department of Physics and Astronomy, University of Oklahoma, Norman, OK, USA

${ }^{114}$ Department of Physics, Oklahoma State University, Stillwater, OK, USA 
115 Palacký University, RCPTM, Olomouc, Czech Republic

${ }^{116}$ Center for High Energy Physics, University of Oregon, Eugene, OR, USA

${ }^{117}$ LAL, Université Paris-Sud and CNRS/IN2P3, Orsay, France

${ }^{118}$ Graduate School of Science, Osaka University, Osaka, Japan

${ }^{119}$ Department of Physics, University of Oslo, Oslo, Norway

${ }^{120}$ Department of Physics, Oxford University, Oxford, UK

121 (a) INFN Sezione di Pavia, Pavia, Italy; ${ }^{(b)}$ Dipartimento di Fisica, Università di Pavia, Pavia, Italy

122 Department of Physics, University of Pennsylvania, Philadelphia, PA, USA

${ }^{123}$ National Research Centre "Kurchatov Institute" B.P.Konstantinov, Petersburg Nuclear Physics Institute, St. Petersburg, Russia

124 (a) INFN Sezione di Pisa, Pisa, Italy; ${ }^{(b)}$ Dipartimento di Fisica E. Fermi, Università di Pisa, Pisa, Italy

125 Department of Physics and Astronomy, University of Pittsburgh, Pittsburgh, PA, USA

126 (a) Laboratório de Instrumentação e Física Experimental de Partículas-LIP, Lisbon, Portugal; ${ }^{(b)}$ Faculdade de Ciências, Universidade de Lisboa, Lisbon, Portugal; ${ }^{(c)}$ Department of Physics, University of Coimbra, Coimbra,

Portugal; ${ }^{(d)}$ Centro de Física Nuclear da Universidade de Lisboa, Lisbon, Portugal; ${ }^{(e)}$ Departamento de Fisica,

Universidade do Minho, Braga, Portugal; ${ }^{(f)}$ Departamento de Fisica Teorica y del Cosmos and CAFPE, Universidad de

Granada, Granada, Spain; ${ }^{(\mathrm{g})}$ Dep Fisica and CEFITEC of Faculdade de Ciencias e Tecnologia, Universidade Nova de

Lisboa, Caparica, Portugal

${ }^{127}$ Institute of Physics, Academy of Sciences of the Czech Republic, Prague, Czech Republic

${ }^{128}$ Czech Technical University in Prague, Prague, Czech Republic

${ }^{129}$ Faculty of Mathematics and Physics, Charles University in Prague, Prague, Czech Republic

${ }^{130}$ State Research Center Institute for High Energy Physics, Protvino, Russia

${ }^{131}$ Particle Physics Department, Rutherford Appleton Laboratory, Didcot, UK

132 (a) INFN Sezione di Roma, Rome, Italy; ${ }^{(\mathrm{b})}$ Dipartimento di Fisica, Sapienza Università di Roma, Rome, Italy

133 (a) INFN Sezione di Roma Tor Vergata, Rome, Italy; (b) Dipartimento di Fisica, Università di Roma Tor Vergata, Rome, Italy

134 (a) INFN Sezione di Roma Tre, Rome, Italy; ${ }^{(b)}$ Dipartimento di Matematica e Fisica, Università Roma Tre, Rome, Italy

135 (a) Faculté des Sciences Ain Chock, Réseau Universitaire de Physique des Hautes Energies-Université Hassan II,

Casablanca, Morocco; ${ }^{(b)}$ Centre National de l'Energie des Sciences Techniques Nucleaires, Rabat, Morocco; ${ }^{\left({ }^{(c)}\right.}$ Faculté

des Sciences Semlalia, Université Cadi Ayyad, LPHEA-Marrakech, Marrakech, Morocco; ${ }^{(d)}$ Faculté des Sciences,

Université Mohamed Premier and LPTPM, Oujda, Morocco; ${ }^{(e)}$ Faculté des Sciences, Université Mohammed V-Agdal,

Rabat, Morocco

136 DSM/IRFU (Institut de Recherches sur les Lois Fondamentales de l'Univers), CEA Saclay (Commissariat à l'Energie

Atomique et aux Energies Alternatives), Gif-sur-Yvette, France

${ }^{137}$ Santa Cruz Institute for Particle Physics, University of California Santa Cruz, Santa Cruz, CA, USA

${ }^{138}$ Department of Physics, University of Washington, Seattle, WA, USA

${ }^{139}$ Department of Physics and Astronomy, University of Sheffield, Sheffield, UK

140 Department of Physics, Shinshu University, Nagano, Japan

${ }^{141}$ Fachbereich Physik, Universität Siegen, Siegen, Germany

142 Department of Physics, Simon Fraser University, Burnaby, BC, Canada

${ }^{143}$ SLAC National Accelerator Laboratory, Stanford, CA, USA

144 (a) Faculty of Mathematics, Physics and Informatics, Comenius University, Bratislava, Slovak Republic; (b) Department of Subnuclear Physics, Institute of Experimental Physics of the Slovak Academy of Sciences, Kosice, Slovak Republic

145 (a) Department of Physics, University of Cape Town, Cape Town, South Africa; ${ }^{(b)}$ Department of Physics, University of Johannesburg, Johannesburg, South Africa; ${ }^{(c)}$ School of Physics, University of the Witwatersrand, Johannesburg, South Africa

146 (a) Department of Physics, Stockholm University, Stockholm, Sweden; ${ }^{(b)}$ The Oskar Klein Centre, Stockholm, Sweden

${ }^{147}$ Physics Department, Royal Institute of Technology, Stockholm, Sweden

148 Departments of Physics and Astronomy and Chemistry, Stony Brook University, Stony Brook, NY, USA

149 Department of Physics and Astronomy, University of Sussex, Brighton, UK

${ }^{150}$ School of Physics, University of Sydney, Sydney, Australia

${ }^{151}$ Institute of Physics, Academia Sinica, Taipei, Taiwan

152 Department of Physics, Technion: Israel Institute of Technology, Haifa, Israel 
153 Raymond and Beverly Sackler School of Physics and Astronomy, Tel Aviv University, Tel Aviv, Israel

${ }^{154}$ Department of Physics, Aristotle University of Thessaloniki, Thessaloníki, Greece

${ }^{155}$ International Center for Elementary Particle Physics and Department of Physics, The University of Tokyo, Tokyo, Japan

${ }^{156}$ Graduate School of Science and Technology, Tokyo Metropolitan University, Tokyo, Japan

157 Department of Physics, Tokyo Institute of Technology, Tokyo, Japan

${ }^{158}$ Department of Physics, University of Toronto, Toronto, ON, Canada

159 (a) TRIUMF, Vancouver, BC, Canada; ${ }^{(b)}$ Department of Physics and Astronomy, York University, Toronto, ON, Canada

${ }^{160}$ Faculty of Pure and Applied Sciences, University of Tsukuba, Tsukuba, Japan

${ }^{161}$ Department of Physics and Astronomy, Tufts University, Medford, MA, USA

162 Centro de Investigaciones, Universidad Antonio Narino, Bogotá, Colombia

${ }^{163}$ Department of Physics and Astronomy, University of California Irvine, Irvine, CA, USA

164 (a) INFN Gruppo Collegato di Udine, Sezione di Trieste, Udine, Italy; ${ }^{\left({ }^{b}\right)}$ ICTP, Trieste, Italy; ${ }^{(c)}$ Dipartimento di Chimica

Fisica e Ambiente, Università di Udine, Udine, Italy

165 Department of Physics, University of Illinois, Urbana, IL, USA

${ }^{166}$ Department of Physics and Astronomy, University of Uppsala, Uppsala, Sweden

${ }^{167}$ Instituto de Física Corpuscular (IFIC) and Departamento de Física Atómica, Molecular y Nuclear and Departamento de Ingeniería Electrónica and Instituto de Microelectrónica de Barcelona (IMB-CNM), University of Valencia and CSIC, Valencia, Spain

168 Department of Physics, University of British Columbia, Vancouver, BC, Canada

169 Department of Physics and Astronomy, University of Victoria, Victoria, BC, Canada

170 Department of Physics, University of Warwick, Coventry, UK

171 Waseda University, Tokyo, Japan

172 Department of Particle Physics, The Weizmann Institute of Science, Rehovot, Israel

${ }^{173}$ Department of Physics, University of Wisconsin, Madison, WI, USA

${ }^{174}$ Fakultät für Physik und Astronomie, Julius-Maximilians-Universität, Würzburg, Germany

${ }^{175}$ Fachbereich C Physik, Bergische Universität Wuppertal, Wuppertal, Germany

${ }^{176}$ Department of Physics, Yale University, New Haven, CT, USA

177 Yerevan Physics Institute, Yerevan, Armenia

${ }^{178}$ Centre de Calcul de l'Institut National de Physique Nucléaire et de Physique des Particules (IN2P3), Villeurbanne, France

a Also at Department of Physics, King's College London, London, UK

${ }^{\mathrm{b}}$ Also at Institute of Physics, Azerbaijan Academy of Sciences, Baku, Azerbaijan

${ }^{c}$ Also at Novosibirsk State University, Novosibirsk, Russia

d Also at TRIUMF, Vancouver, BC, Canada

e Also at Department of Physics, California State University, Fresno, CA, USA

${ }^{f}$ Also at Department of Physics, University of Fribourg, Fribourg, Switzerland

g Also at Departamento de Fisica e Astronomia, Faculdade de Ciencias, Universidade do Porto, Porto, Portugal

${ }^{\mathrm{h}}$ Also at Tomsk State University, Tomsk, Russia

${ }^{i}$ Also at CPPM, Aix-Marseille Université and CNRS/IN2P3, Marseille, France

j Also at Universita di Napoli Parthenope, Naples, Italy

${ }^{k}$ Also at Institute of Particle Physics (IPP), Waterloo, Canada

${ }^{1}$ Also at Particle Physics Department, Rutherford Appleton Laboratory, Didcot, UK

${ }^{m}$ Also at Department of Physics, St. Petersburg State Polytechnical University, St. Petersburg, Russia

${ }^{\mathrm{n}}$ Also at Louisiana Tech University, Ruston, LA, USA

${ }^{\circ}$ Also at Institucio Catalana de Recerca i Estudis Avancats, ICREA, Barcelona, Spain

p Also at Graduate School of Science, Osaka University, Osaka, Japan

${ }^{q}$ Also at Department of Physics, National Tsing Hua University, Taiwan

${ }^{\mathrm{r}}$ Also at Department of Physics, The University of Texas at Austin, Austin, TX, USA

${ }^{s}$ Also at Institute of Theoretical Physics, Ilia State University, Tbilisi, Georgia

${ }^{t}$ Also at CERN, Geneva, Switzerland

u Also at Georgian Technical University (GTU), Tbilisi, Georgia

${ }^{v}$ Also at Manhattan College, New York, NY, USA

${ }^{w}$ Also at Hellenic Open University, Patras, Greece 
${ }^{\mathrm{x}}$ Also at Institute of Physics, Academia Sinica, Taipei, Taiwan

y Also at LAL, Université Paris-Sud and CNRS/IN2P3, Orsay, France

${ }^{\mathrm{z}}$ Also at Academia Sinica Grid Computing, Institute of Physics, Academia Sinica, Taipei, Taiwan

aa Also at School of Physics, Shandong University, Shandong, China

${ }^{a b}$ Also at Moscow Institute of Physics and Technology State University, Dolgoprudny, Russia

${ }^{a c}$ Also at Section de Physique, Université de Genève, Geneva, Switzerland

${ }^{\text {ad }}$ Also at International School for Advanced Studies (SISSA), Trieste, Italy

ae Also at Department of Physics and Astronomy, University of South Carolina, Columbia, SC, USA

${ }^{\text {af }}$ Also at School of Physics and Engineering, Sun Yat-sen University, Guangzhou, China

ag Also at Faculty of Physics, M.V.Lomonosov Moscow State University, Moscow, Russia

ah Also at National Research Nuclear University MEPhI, Moscow, Russia

ai Also at Department of Physics, Stanford University, Stanford, CA, USA

aj Also at Institute for Particle and Nuclear Physics, Wigner Research Centre for Physics, Budapest, Hungary

${ }^{\text {ak }}$ Also at Department of Physics, The University of Michigan, Ann Arbor, MI, USA

${ }^{\text {al }}$ Also at University of Malaya, Department of Physics, Kuala Lumpur, Malaysia

* Deceased 